\title{
BUCHSTABEN DER WELT
}

\section{WELT DER BUCHSTABEN}

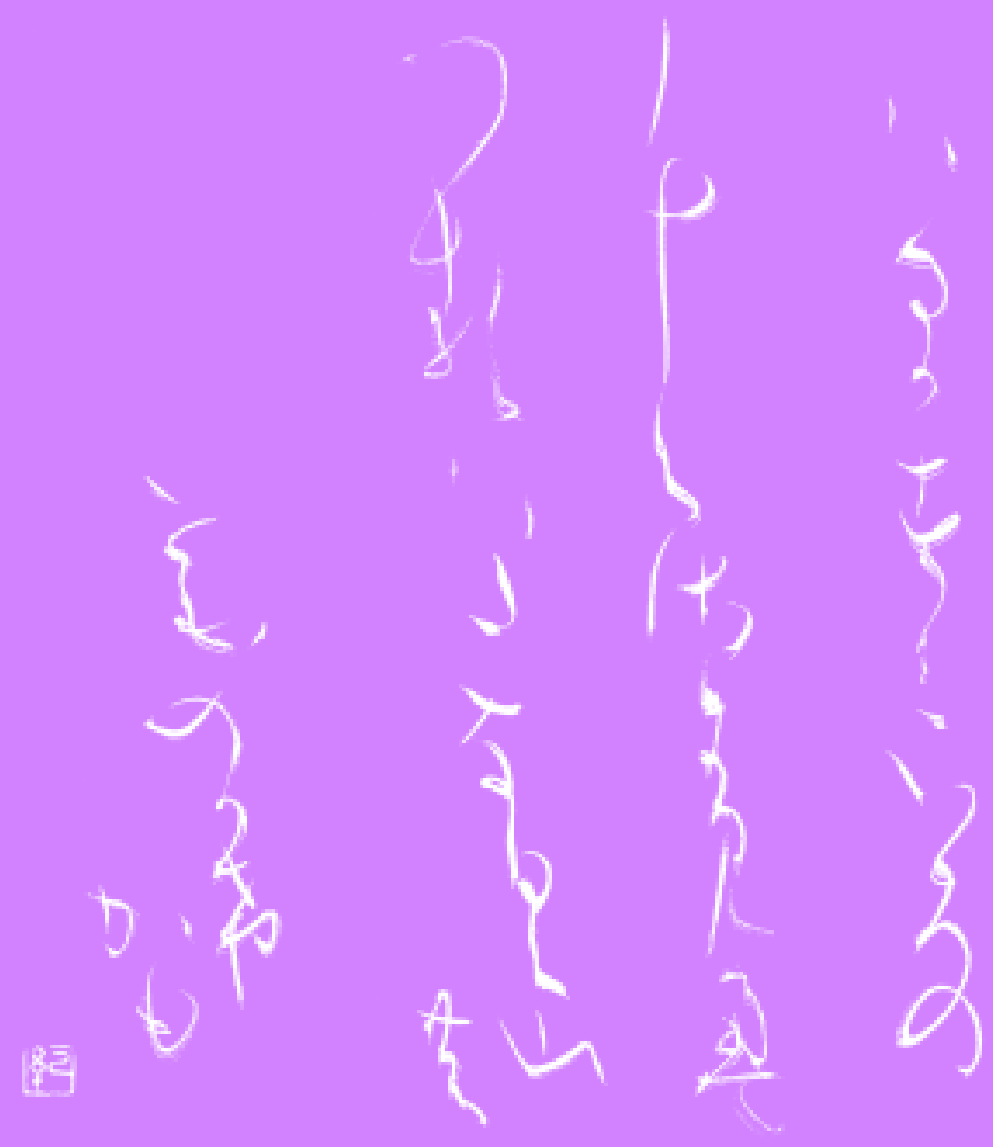

MORPHOMATA 
In der Gestalt von Buchstaben und anderen Schriftzeichen manifestieren sich Denktraditionen und Schreibhorizonte: Indem Buchstaben Zeichen figurieren, machen sie implizit kulturelles Wissen lesbar. Der Unicode-Standard bietet heute die umfassendste Codierung in der Kultur der Menschheit; das Verhältnis von universeller Lesbarkeit und der phänomenalen Gestalt prinzipiell aller Schriftzeichen wird hier neu verhandelt. In Zeiten weltweiter Kommunikationstechnologien stellt sich auf der einen Seite die Frage nach kulturellen und phänomenalen Besonderheiten von „Buchstaben der Welt" auch in vergleichender Hinsicht daher neu: Wie entstehen die Schriftzeichen, die wir schreiben, das heißt, was sind die technischen, semiotischen, linguistischen oder ästhetischen Bedingungen für ihre Form in verschiedenen Sprachsystemen? Auf der anderen Seite eröffnet sich eine „Welt der Buchstaben" im Sinne einer Innenwelt, die phänomenologischphilosophisch analysiert werden kann. - Beiträge, die philosophische Denkräume und die Erscheinungsformen von Kultur als Buchstaben ausloten, bilden einen Schwerpunkt des Bandes. Komparative Betrachtungen zu fernöstlichen Zeichen und Schreibweisen, wie in der japanischen Kalligraphie, erlauben einen erweiterten Blick auf die unterschiedlichen Ausformungen von Zeichen und hiermit verbundene Funktionen. Grundlegende Erörterungen zur Buchstabengestalt runden den Band ab: aus Sicht der Semiologie, der Linguistik und des Schriftgestalters. 
ÔHASHI, ROUSSEL (HRSG.) -

BUCHSTABEN DER WELT - WELT DER BUCHSTABEN 


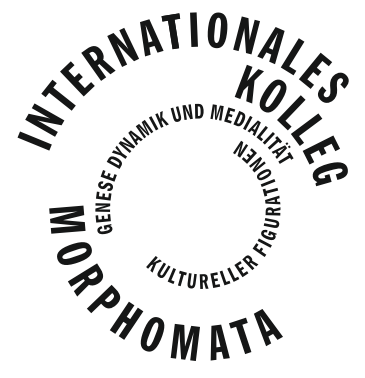

\section{MORPHOMATA}

HERAUSGEGEBEN VON GÜNTER BLAMBERGER UND DIETRICH BOSCHUNG

BAND 12 
HERAUSGEGEBEN VON RYÔSUKE ÔHASHI

UND MARTIN ROUSSEL

\section{BUCHSTABEN DER WELT \\ WELT DER BUCHSTABEN}

WILHELM FINK 
GEFÖRDERT VOM

Bundesministerium

für Bildung

und Forschung

unter dem Förderkennzeichen 01UK0905. Die Verantwortung für den Inhalt der Veröffentlichung liegt bei den Autoren.

Bibliografische Informationen der Deutschen Nationalbibliothek: Die Deutsche Nationalbibliothek verzeichnet diese Publikation in der Deutschen Nationalbibliografie; detaillierte Daten sind im Internet über www.dnb.d-nb.de abrufbar.

Alle Rechte, auch die des auszugweisen Nachdrucks, der fotomechanischen Wiedergabe und der Übersetzung vorbehalten. Dies betrifft auch die Vervielfältigung und Übertragung einzelner Textabschnitte, Zeichnungen oder Bilder durch alle Verfahren wie Speicherung und Übertragung auf Papier, Transparente, Filme, Bänder, Platten und andere Medien, soweit es nicht $\ 53$ und 54 UrhG ausdrücklich gestatten.

(C) 2014 Wilhelm Fink Verlag, München

Wilhelm Fink GmbH \& Co. Verlags-KG, Jühenplatz 1, D-33098 Paderborn

Internet: www.fink.de

Lektorat: Martin Roussel

Gestaltung und Satz: Kathrin Roussel, Sichtvermerk

Printed in Germany

Herstellung: Ferdinand Schöningh GmbH \& Co. KG, Paderborn

ISBN 978-3-7705-5609-0 


\section{INHALT}

RYÔSUKE ÔHASHI UND MARTIN ROUSSEL

Einleitung

\section{WAS IST EIN BUCHSTABE?}

BEATRICE PRIMUS UND LUTZ WAGNER Buchstabenkomposition 33

STEFAN CLAUDIUS Schriftproduktion heute 59

CHRISTIAN STETTER Buchstabe und Zeichen $\quad 87$

\section{BUCHSTABEN DER WELT}

HELWIG SCHMIDT-GLINTZER Eine Schrift, die keiner kennt.

Ostasiatische Schriftzeichen und das „Alphabet der Bitternis“ -

Bemerkungen zum Traum von einer Begriffsschrift

RYOZO MAEDA Symbolisch-Imaginär? Manga und die ,kulturfigurative“ Wirkung der japanischen Schrift

NORIKO KAYA The Aesthetic Characteristics of Kana

seen in Chirashi-gaki

MATHIAS OBERT Chinesische Schrift und leibliches Denken

\section{WELT DER BUCHSTABEN}

YVONNE FÖRSTER-BEUTHAN Versuch einer Phänomenologie

des Buchstabens

RALF BEUTHAN Vom Geist der Buchstaben.

Medienphilosophische Überlegungen im Anschluss an Hegel 
EVELINE CIOFLEC Schrift als Sinnstiftung.

Eine soziokulturelle Fragestelltung

Beiträgerinnen und Beiträger 
RYÔSUKE ÔHASHI UND MARTIN ROUSSEL

\section{EINLEITUNG}

I. VOM BILD ZUR SCHRIFT: AUSGANGSPUNKTE

Ryôsuke Ôhashi

Im Folgenden versucht der Verfasser als Initiator des Workshops „Buchstaben der Welt - Welt der Buchstaben“, auf den dieses Buch zurückgeht, sein anfängliches Motiv kurz darzulegen. Dies konnte nicht in Form eines Beitrags zum Workshop ausgearbeitet werden, wurde aber im Verlauf der Veranstaltung zu seiner Freude weit über die Erwartungen hinaus entwickelt. Jetzt, wo die Ergebnisse des Workshops als Tagungsband erscheinen, darf vielleicht auch das ursprüngliche Motiv als Ausgangspunkt dokumentiert werden. An diese hinführenden Worte knüpfen die Erläuterungen von Martin Roussel an.

Der Verfasser war von einer Frage ausgegangen: Inwieweit können Buchstaben nicht nur aus dem historischen Kontext ihrer antiken Prägung, sondern auch bis heute in der globalisierten Welt als spezifische Ausformungen des Wissens, mithin als ,Morphome ${ }^{{ }^{1}}$ bestimmt werden? In der östlichen Welt und in der westlichen Welt der Antike entstanden nämlich die unterschiedenen Arten von Buchstaben oder Schriftzeichen ohne wechselseitigen Einfluss aufeinander. Sie waren in vielen Hinsichten unterschieden, jedoch könnte man man anhand ihres Charakters als Buchstaben bzw. Schriftzeichen, also im Sinne des englischen character, spezifische Eigenheiten, wenn nicht die Ganzheit der jeweiligen Geschichts- und Kulturwelten ans Licht bringen. Buchstaben können dabei

1 Das Wort Morphom stellt eine Ableitung von gr. mórphoma dar, pl. morphómata und bezeichnet die in einem Gestaltungsprozess manifestierte Form oder Figur. Vgl. Günter Blamberger, Dietrich Boschung (Hrsg.): Morphomata. Kulturelle Figurationen: Genese, Dynamik und Medialität. München 2011 (Morphomata; 1). 
auch selber als kreative Orte begriffen werden, an denen Kultur gestaltet wird, und bleiben nicht bloße Teilphänomene der jeweiligen Kulturen.

Die Welt heute gilt aber als globalisiert, so dass die Kulturen sich im regen Verkehr überschneiden, ineinander führen und sich gegenseitig beeinflussen, so dass kaum ein Phänomen eines Kulturkreises für einen anderen dauerhaft unbekannt bliebe. Gelten die Phänomene der Buchstaben heute noch als ,Morphome' im oben genannten Sinne? Ohne diesen heutigen Aspekt bleibt die Feststellung der Buchstaben als Morphome nur retrospektiv. Die Betrachtung der Buchstaben sollte und könnte ermöglichen, die Phänomenalität des Buchstabens in der Gegenwart neu zu beleuchten und von hier aus rückblickend auch die vergangenen bzw. alten Buchstabenphänomene anders zu interpretieren als in der konventionellen Buchstabengeschichte. Inwieweit wäre eine solche Aussicht in einem Workshop und darüber hinaus im Buchformat zu eröffnen?

Um diese Frage als Anfangsmotiv zu bearbeiten, fand der Verfasser einen Ansatzpunkt in einer japanischen Kunstform, die jetzt, nachträglich, vorgestellt wird.

In der Kunstgeschichte ist bekannt, dass auf asiatischen Tuschebildern oft mit Schriftzeichen geschriebene Gedichte aufgeschrieben werden. Dieser Stil hat sich besonders in der japanischen emakimono (wörtlich: Bildrolle) entwickelt. Meistens arbeiten zwei Künstler zusammen, der eine als Maler und der andere als Kalligraph. Ein ausgezeichnetes Beispiel dafür ist: Shiki-sôka-shitae-kokin-sĥu (Sammlung-der-Gedichte-von-einstund-jetzt-mit-unterlegten-Bildern-von-Pflanzen-der-vier-Jahreszeiten). Der Maler Sôtatsu (geboren in der letzten Hälfte des 16. und tätig in der ersten Hälfte des 17. Jahrhunderts) und der Kalligraph Hon'ami Kôetsu (1558-1637) arbeiteten für dieses Werk gemeinsam, wie des Öfteren. Durch ihre Zusammenarbeit entstanden prachtvolle Rollbilder, die in ihrer Art unübertroffen sind. Beide Künstler, welche die bürgerliche Kultur in Kyoto am Ende des 16. Jahrhunderts repräsentieren, wetteiferten als Genies des Kunsthandwerks miteinander in jeweils verschiedenen Künsten.

Nur ein Bild ist aus diesem Werk heranzuziehen, obwohl alle Bilder in dieser Rolle als Beispiele für das Thema des Verfassers geeignet sind. Dort werden Blätter und Ranken von Efeu dargestellt. 


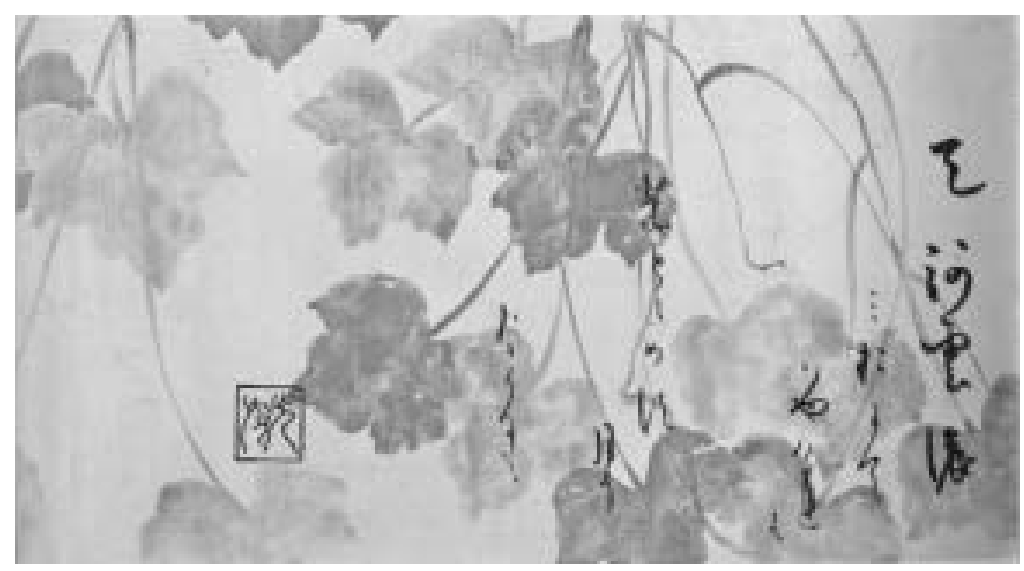

1 Tawaraja Sôtatsu und Hon'ami Kôetsu: Shiki-sôka-shitae-kokin-sĥ̂

Die Ranken hängen in fließenden Linien von oben in das Bild hinein. Über den Ranken lässt Kôetsu seinen Pinsel spielen, um ein Gedicht kalligraphisch zu gestalten. Das Gedicht wurde im 10. Jahrhundert in die Gedichtsammlung Kokin-waka-sh $\hat{u}$ (Sammlung-der-japanischenKurzgedichte-von-einst-und-jetzt) aufgenommen, die durch kaiserlichen Erlass offiziell erstellt wurde. Das Gedicht, dessen Verfasser unbekannt ist, lautet:

Ama no kawa / kumo no mio nite / hayakereba / hikari todomezu / tsuki zo nagaruru (Wenn am Nachthimmel / die Wolkenketten auf ihren Bahnen / so rasch dahinjagen, / scheint selbst der Mond dahinzutreiben / mit seinem wechselnden Licht.)

Liegt der künstlerische Brennpunkt dieses Rollbildes im gemalten Bild oder in gedichteten Sätzen - oder in der bildhaften Kalligraphie?

Sicherlich sind die unvergleichliche Harmonie, zugleich aber auch der Wettstreit von kalligraphischen Tuschelinien, von Blattgold und -silber der erste Eindruck, den die Bildrolle vermittelt. Somit ist zunächst die visuelle Gestaltung vorherrschend. Die Spur der Tusche ist jedoch nicht nur eine visuelle Gestaltung, sondern auch der Ausdruck des Gedichts. Wird der ,Sinn' des Gedichts verstanden, kommt eine Dimension der Bilder zum Vorschein, die bis dahin verborgen war. Wenn sich die kalligraphische Linie in Form des Schriftzeichens für ,Mondlicht' über den Efeu erstreckt, beginnt das Mondlicht zwischen den treibenden Wolken hindurchzuscheinen und wirft einen sanften Schatten auf die Blätter des Efeus. Der Sinn des Gedichts durchzieht die Kalligraphie 
sowie das gemalte Bild, so dass eine Dimension entsteht, in der das diskontinuierliche Kontinuum von Bild und Schrift sichtbar wird. Indem die kallligraphische Linie einen eigenen Sinn gewinnt und als gestaltete Schrift aufgeht, steht sie nicht mehr dem Bild nach, dem in der Welt des Sehens für gewöhnlich der Vorrang gegeben wird. Das Bild wird einer Literarisierung unterworfen. In der Welt der Bedeutungen, die sich nun zeigt, wird die Schrift zur Hauptsache, und das Bild folgt ihr, womit die bisherige Beziehung umgekehrt wird. Um die Bedeutung des Gedichtes malerisch auszudrücken, darf das Bild natürlich nicht einfach literarisiert bleiben. So regt wiederum umgekehrt das Bild die Vorstellung an und will die Schrift verbildlichen.

Bild und Schrift trachten hier danach, sich gegenseitig ihre Seinsweise zu entreißen. Das gelungene Ringen der beiden geht nicht einfach dadurch zu Ende, dass die eine Seite gewinnt. Denn die geraubte eigene Seinsweise geht in die andere Seinsweise über und verändert diese.?

Ein anderer und fundierender Grund für dieses künstlerische Ringen von Bild und Schrift bei Sôtatsu und Kôetsu war offensichtlich dies: dass Schrift mit den sinn-bedeutenden und bildhaften Buchstaben, das heißt Schriftzeichen, geschrieben wird. Der Bildcharakter der europäischen Alphabete ist nicht in der gleichen Weise bemerkenswert, dass ihre kalligraphische Gestaltung über den Illustrationscharakter hinaus mit dem malerisch dargestellten Bild konkurrieren würde.

In diesem Zusammenhang ist aber daran zu erinnern, dass in der Buchmalerei des Mittelalters bis zur Renaissance-Zeit die handschriftlich geschriebenen Texte mit Grundfarben und Tönen verziert wurden, so dass die Textgestaltung einen prachtvollen Bildcharakter erhielt. Das Ganze wurde zwar letzthinnig für die ,Lektüre' konzipiert, aber die Mehrheit der Bevölkerung von damals waren Analphabeten und konnte die heiligen Texte nicht selber lesen. Diese sollten nicht durch den geschriebenen

2 Bis hierher ist die Darstellung eine Verkürzung eines Abschnitts des Verfassers in seinem Buch Kire. Das, Schöne' in Japan. Philosophischästhetische Reflexionen zu Geschichte und Moderne (Köln 1994, 2. Aufl. im Druck, S. 111-114). Das Wort Kire heißt auf Deutsch: ,Schnitt‘. Es geht um das ,Schnitt-Kontinuum“ bzw. die diskontinuierliche Kontinuität, wie sie in der japanischen Kunst als deren Grundstruktur in verschiedener Weise zu finden ist. Im vorliegenden Beispiel geht es um das ,Schnitt-Kontinuum“ von Bild und Schrift bzw. Buchstaben. 
Inhalt, sondern durch die Verzierung ihrer Gestalt das Gefühl der Verehrung erwecken. Dies ist ein Indiz dafür, dass die Bedeutung der Buchstaben sich nicht in der Lesefunktion erschöpft.

In der Malerei der Renaissance kann man in dieser Hinsicht andere Beispiele finden, wie zum Beispiel Albrecht Dürer. Er hat im Portrait seiner Mutter und in seinen Selbstportraits kalligraphisch gestaltete Sätze aufgeschrieben. Sehen wir die Inschrift auf dem 1493 entstandenen Selbstportrait:

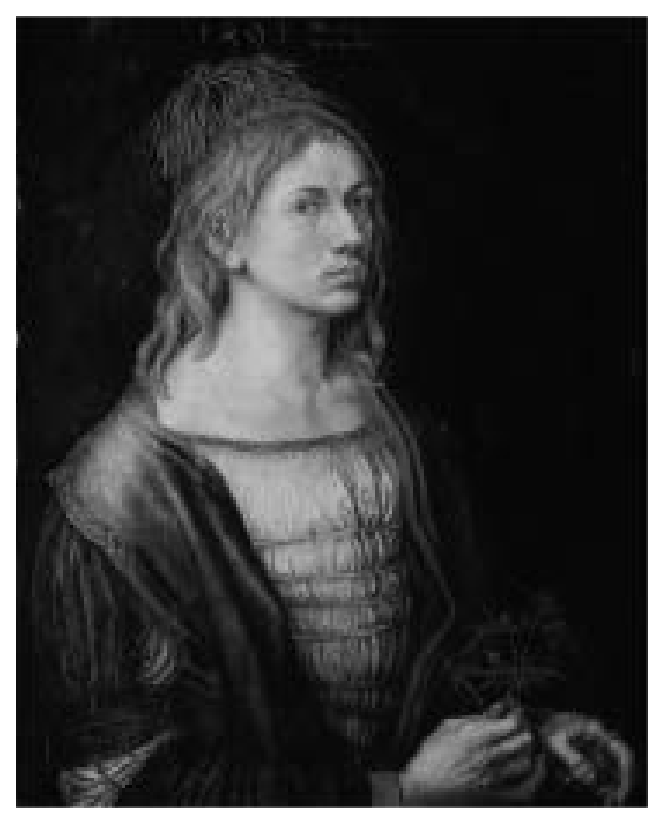

2 Albrecht Dürer: Selbstbildnis mit Eryngium (1493)

Dort schreibt Dürer handschriftlich einen Satz: „My sach die gat / Als es oben schtat". Grob umschrieben lautet der Satz: Meine Sache ist so, / wie oben im Himmel bestimmt wird. Der Maler stellt sich selbst dar, wobei er sich dessen bewusst war, dass er der himmlischen Bestimmung unterworfen wird. Es ist typisch für die Genies der Renaissance, dass sie sich selbst wegen ihrer genialen Begabung für göttlich hielten. Um diese göttliche Menschennatur künsterlich zum Ausdruck zu bringen, hatte Dürer ein Bedürfnis, den obigen Satz auf dem Bild aufzuschreiben. Dies könnte man negativ oder positiv interpretieren: negativ als Mangel der Ausdrucksfähigkeit der Malerei, die der Ergänzung durch die Buchstaben 
bedarf; positiv als Beleg dafür, dass Bild und Schrift, Figur und Buchstaben, nicht schroff voneinander getrennt, sondern eine geteilte Beschaffenheit besitzen, so dass ein produktives Zusammenwirken beider geschehen kann. Allerdings bleiben in diesem Zusammenwirken bei Dürer die Buchstaben eher ein bescheidenes Hilfsmittel für die Malerei, statt mit dieser ein künsterlisches, Ringen' zu leisten.

Ein modernes Beispiel ist bei Paul Klee zu finden, wenn dieser die alphabetischen Buchstaben zum malerischen Motiv macht. Die eigentliche Funktion der Buchstaben, die Sinnbildung und -vermittlung wird durch die ,Verbildlichung ihrer Gestalt minimalisiert. Diese Bilder Klees lassen uns erneut daran denken, dass die zunächst mit keinen Sinnbedeutungen beladenen alphabetischen Buchstaben, diese atomistischen Elemente der sinnbildenden Sätze, zugleich bildhaft und figurativ sind. Die Forschung zur Entstehung der alphabetischen Buchstaben kann belegen, dass und wie ihre Entstehung mit diesem Element des Bildhaften verbunden ist.

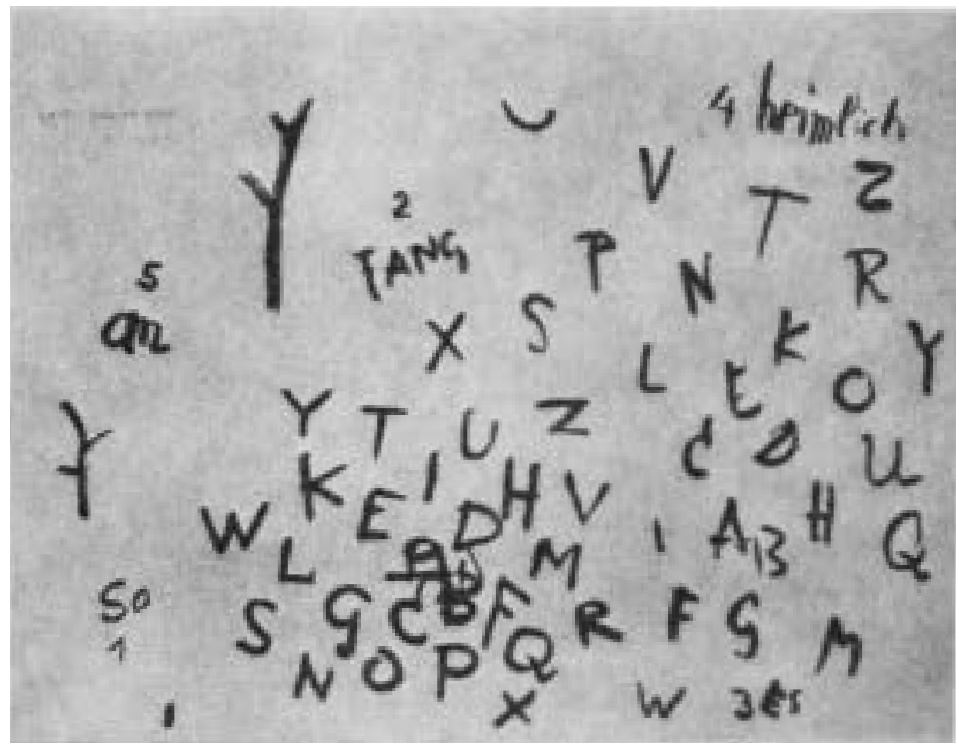

3 Paul Klee: Anfang eines Gedichtes (1938)

Es wäre hier nicht unnütz, dieses Phänomen der Verbildlichung der Buchstaben vielleicht auch in einer anderen Perspektive zu beleuchten, wie es Derrida in seiner Grammatologie im Hinblick auf die Phonetisierung der Schrift und die Herrschaft des logozentrischen Ethnozentrismus 
in der europäschen Welt erschließt. Derrida sieht, dass „die Phonetisierung der Schrift mit ihrem Entstehen ihre eigene Geschichte verbergen muß “. ${ }^{3}$ Diese eigene Geschichte bzw. der Ursprung der Schrift wird nach Derrida nicht von dem erlebt, der die Schrift als das dem Schreiben und Lesen zugehörige Selbstverständliche nimmt. Wenn man die Schrift schreibt und liest, „sieht“ er sie nicht, sagt Derrida.

Man muß sich stets daran erinnern, daß das Wort, die ,Vokabel', gehört wird: das stimmliche Phänomen als solches bleibt unsichtbar. Sofern das Wort in uns eher eine Zeit als einen Raum besetzt, richtet es sich nicht bloß - als Code für Nicht-Sehende - von Blinden an Blinde, sondern spricht zu uns in Wahrheit die ganze Zeit über von der Blindheit, die es konstituiert. ${ }^{4}$

Mit dem Ausdruck Heideggers „die Sprache spricht“ fährt Derrida fort: „Die Sprache spricht, sie spricht von sich, d. h. sie spricht von der Blindheit. Stets spricht sie zu uns von der Blindheit (her), die sie konstituiert.“5 Der die Schrift Schreibende bzw. Lesende ist in Wahrheit ein ,Blinder‘. Gerade aber dadurch, dass er sich im Schreiben und Lesen der Schrift als Blinder weiß, erlebt er (somit ,sieht' er), was die Schrift ist.

Derridas Blick auf die Problematik der Phonetisierung der Buchstaben in Europa ist zwar nicht auf das Durchdringen des Bildcharakters im ostasiatischen Schreiben gerichtet. Doch wenn Derrida in seiner Betrachtung so weit geht zu sagen, dass die Sprache des Wortes von einer ,Blindheit' spricht, so wäre das Durchdringen von Schrift und Bild in Ostasien auch bei ihm in eigentümlicher Weise mit gedacht. Seine Grammatologie kann insofern für unser Thema herangezogen werden. Die Ausführung dieser Ahnung möchte der Verfasser allerdings den folgenden Überlegungen Martin Roussels überlassen.

In der Betrachtung des Durchdringens bzw. des diskontinuierlichen Kontinnums von Schrift und Bild im Fernen Osten sollten und könnten desweiteren zwei moderne Kulturphänomene ins Auge fallen: Das eine sind die in den europäischen Städten überall zu sehenden ,Graffiti‘

3 Jacques Derrida: Grammatologie (1967). Übersetzt von Hans-Jörg Rheinberger und Hanns Zischler. Frankfurt a. M. 1974, S. 11.

4 Jacques Derrida: Aufzeichnungen eines Blinden. Das Selbstporträt und andere Ruine. Hrsg. von Michael Wetzel. Aus dem Französischen von Andreas Knop und Michael Wetzel. München 1997, S. 11.

5 Ebd. 
und das andere die in Japan entstandenen, inzwischen aber weltweit bekannt gewordenen ,Mangas'. In beiden Phänomenen findet man, dass die Buchstaben in karikiertem Stil verbildlicht werden und die Bilder einen Aussage-Charakter erhalten, der sonst mit Buchstaben ermöglicht wird. Die bisher als selbstverständlich vorgestellte Scheidelinie zwischen Schriftzeichen und Bildern kann hier so sehr fraglich werden, dass diese beiden Phänomene verschmelzen.

Was ,Graffiti' betrifft, so ist hier von der Kontroverse abzusehen, ob und inwieweit sie als ,Kunst ${ }^{\star}$ anzusehen seien, ob es den kategorialen Unterschied zwischen den hohen, künstlerischen und den gemeinen, häßlichen Graffiti gibt usw. Es kommt hier nur darauf an, zu sehen, dass in manchen Graffiti-Bildern die Buchstaben großes Gewicht bekommen, wobei zwischen den Buchstaben und den bildhaft bemalten Figuren kein prinzipieller Unterschied mehr zu finden ist.

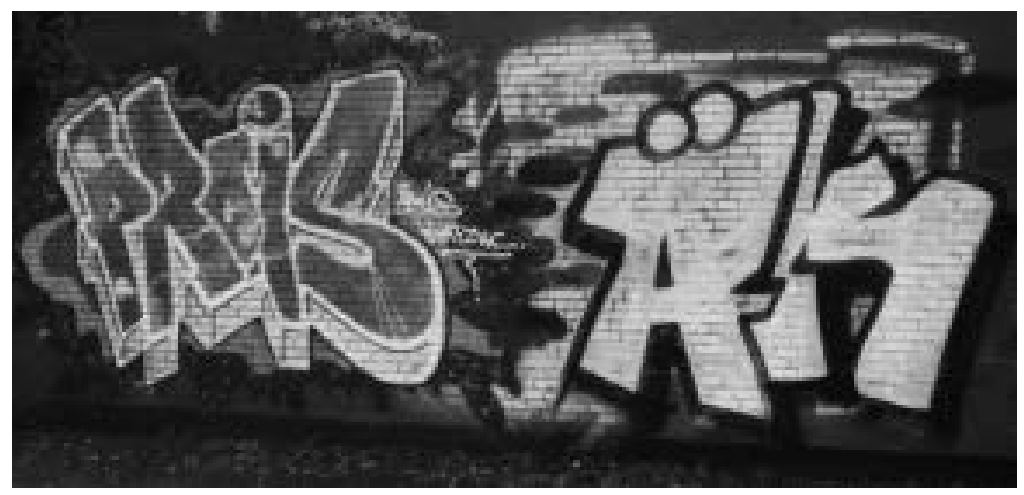

4 Graffito

Die Buchstaben werden bildhaft dargestellt, und die malerischen Figuren haben einen Buchstabencharakter, so dass ein Graffito-Bild im Ganzen mit seinen grotesken Figuren meistens eine Behauptung bzw. einen Propaganda-Charakter erhält.

Die ,Mangas', deren Gründer offensichtlich Hokusai ist, ein Maler aus der Edo-Zeit ${ }^{6}{ }^{6}$ werden heute in allen Erdteilen anerkannt und reproduziert.

6 Hokusai ist mit seiner Publikation Hokusai-Manga (zuerst erschien 1814) der Gründer der Manga-Tradition. Das Wort Manga besteht aus zwei Buchstaben. Man heißt so etwas wie: ,müßig', ,gemütlich', ,ohne ernste Absicht und Zwecke‘; ga heißt: ,Bild‘. Manga heißt also: ,die Bilder, die nur aus Lust ohne besondere Zwecke bemalt werden'. 
Die Verfilmung einiger Mangas, der sogenannten ,Animes', ist weltweit sogar modisch. Im Vergleich mit den Mangas von Hokusai betonen und übertreiben die heutigen noch mehr die dramatisierende Darstellungsart, die vielleicht bei manchen Lesern das Gefühl einer Befreiung von der Alltagswelt gibt. Die Einziehung der Scheidelinie zwischen Schriftzeichen und Bildern wird durch die Serialität der Bilder noch intensiviert. Wenn z. B. die mit den japanischen Alphabeten onomatopoetisch wiedergegebenen Glockentöne Gôn oder der Schrei Gyâ übergroß und mit wiederholten Vokalen "Gôôôôon" oder „Gyââââa..." geschrieben werden, was teilweise auch alphabetisch umsetzbar ist, so sind diese Buchstaben keine Buchstaben im üblichen Sinne mehr, sondern Bilder für das Glockentönen oder den Schrei. Die Graffiti und Mangas veranlassen uns, in Hinsicht auf ihre Gleichzeitigkeit als Weltphänomene in Ost und West und neue Aspekte der Schriftzeichen, diese als Morphome zu betrachten.

$\mathrm{Ob}$ diese Betrachtung nur eine harmlose Feststellung bleibt oder ob durch sie eine neue Perspektive zur Auffassung der Buchstaben geöffnet wird, ist eine Frage, die hier offen gelassen werden darf. Nur als Andeutung dafür ist zum Schluss ein Bild von van Gogh zu zeigen. Einst bildete er ein Werk von Hiroshige nach, das malerisch gerahmt wird, und auf dessen Bildrahmen Hiroshige Schriftzeichen aufgeschrieben hat. Van Gogh bildete auch diesen Rahmen mit den Schriftzeichen nach, ohne deren Wortbedeutung zu kennen.

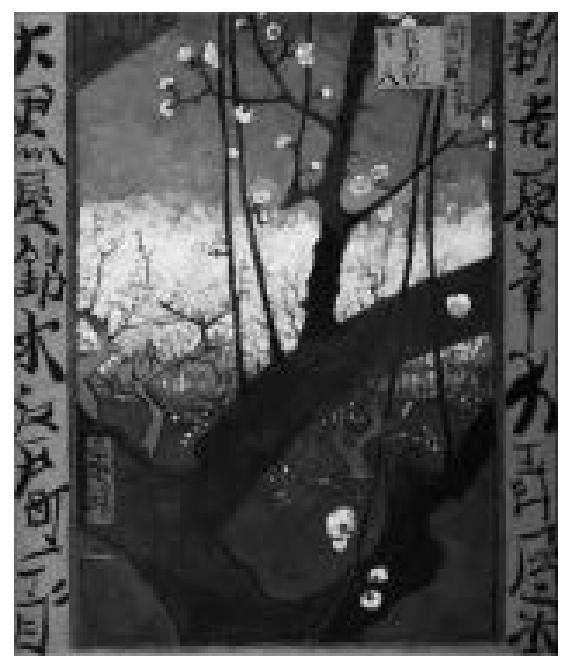

5 Vincent van Gogh: Blühender Pflaumenbaum (nach Hiroshige) (1887) 
Die bemalten Schriftzeichen sind bei van Gogh keine Schriftzeichen mehr - und damit buchstäblich nicht lesbar -, sondern reine Bilder. Für van Gogh geht es letztlich um die im Bild Hiroshiges bemalte ,Welt' Japans, die er malerisch nachbilden wollte. Durch diese Nachbildung kam aber die eigene malerische Welt van Goghs selbst zum Ausdruck. Man sieht in der Tat beim ersten Blick, dass es sich um ein Werk van Goghs handelt. Die „Welt der Buchstaben“ und die „Buchstaben der Welt“ zeigen sich in diesem Werk als ein kreatives Geschehen. Dieses wird allerdings erst im vergleichenden Blick auf die Adaption der Form unabhängig von Fragen der Signifikation eröffnet. Eben hierin erhält die Rede vom Morphom ihren Sinn. 


\section{VON DER SCHRIFT ZUM BILD: ERLÄUTERUNGEN Martin Roussel}

Wie sähe eine Gegenerzählung aus, von der nicht der Buchstabe aus der Bilderwelt anschaulich würde, ${ }^{7}$ sondern deutlich würde, wie der Buchstabe aus der Schrift heraus das Bild erschließt? Ich greife hierfür auf eine geradezu kindlich anmutende Erzählung zurück, die Robert Walser 1921 in der Neuen Zürcher Zeitung unter dem Titel „Alphabet“ veröffentlicht hat.8 Bei dem Alphabet, das im „Alphabet“ als Erzählung ausgestaltet wird, handelt es sich zunächst um das bekannte der abendländischen Tradition. Wie jedoch könnte hieraus der Zündstoff für Walsers Literatur erwachsen, und das heißt: Wie lässt sich das jahrtausendealte Alphabet derart figurieren, dass es die Idiomatik eines kleinen Feuilletontextes signiert? Man kennt Ähnliches aus den ABC-Büchern, wo den Kindern nach der Einschulung die Welt der Schrift - die Welt des Buchstabens - bildlich vermittelt wird, damit sie über den Umweg des Malens das Schreiben lernen. Karl Philipp Moritz etwa, am Beginn dessen, was man das pädagogische Zeitalter nennen könnte, findet in seinem Neuen $A B C$-Buch eine evokative Sprache, um die Situation des Lesens zu verdeutlichen:

7 Die Schrift aus ihrem Verhältnis zum Bildlichen zu verstehen, liegt auch der jüngeren Schriftbildforschung zugrunde, mit der gegenüber der alten Lessing'schen Entgegensetzung von Zeit- (Wort) und Raumkünsten (Malerei) Fragen der - Zwischen- - Räumlichkeit in Katergorien der Schriftanalyse eingetragen werden. Deutlich zeigt dies etwa ein Beitrag Sybille Krämers: Operative Bildlichkeit. Von der,Grammatologie‘ zu einer ,Diagrammatologie‘? Reflexionen über erkennendes ,Sehen', der im Kontext die Schrift eines Bandes zu visuellen Erkenntnisformen zuordnet: Logik des Bildlichen: Zur Kritik der ikonischen Vernunft. Hrsg. von Martina Hessler und Dieter Mersch. Bielefeld, S. 94-122. - „Bilder zu ,schreiben“ und Schriften $\mathrm{zu}$,malen' ist [...] ein konstitutives Prinzip der Kunst des 20. Jahrhunderts" (Schmitz-Emans: Schrift und Abwesenheit. Historische Paradigmen zu einer Poetik der Entzifferung und des Schreibens. München 1995, S. 471).

8 Robert Walser: Das Alphabet. In: ders.: Sämtliche Werke in Einzelausgaben. Hrsg. von Jochen Greven. Zürich und Frankfurt a. M. 1986, Bd. 17, S. 192 f. (Im Folgenden ohne einzelne Zitatnachweise.) 
Erstes Bild

Gesicht

In diesem Buche stehen Bilder und Buchstaben.

Das erste Bild stellt das Auge vor, womit ich die Bilder sehe. ${ }^{9}$

Wichtig dabei: „Bilder und Buchstaben“ müssen unterschieden bleiben, ja, die erste didaktische Lehre besteht darin, in der Evokation des Bildlichen als Umweg zur Schrift sogleich zu verdeutlichen, dass die Bilder nur illustrieren, was in den Buchstaben zur Fähigkeit des Schreibens anleiten soll. Bilder sollen die Lese- und Schreibfähigkeit anleiten, ohne den Dialog mit der Schrift, ohne im Lesen oder im Schreiben den Textfluss, die Erzählung eines Textes, die kognitive Verarbeitung oder den Schreibfluss zu stören. „Bei Moritz gibt es jedenfalls nichts zu lachen, während im ABC-Buch von Jean Pauls Fibel munter vor sich hingereimt werden darf: ,Ein Affe gar possierlich ist. / Zumal, wenn er vom Apfel frisst.““10 Nichts könnte also gefährlicher sein, als die Schrift als ein Buchstabenspiel misszuverstehen. An genau diesem Missverständnis jedoch ist Walser interessiert. Seine Literatur geht gleichsam den Weg zurück zur Fibel, um die Anfangsgründe des Schreibens - ihre assoziative Nähe zum Bild für eine poetische Erzählung vom Buchstaben auszubeuten. Nichts als Buchstaben geben sich in der Geschichte dieses kleinen Textes zu lesen:

Robert Walsers Literatur des Alphabets beginnt: „A. reitet als Amazone durch eine Allee. Was für ein nobler, prächtiger Anblick. Ich könnte mich in die schöne Gestalt beinah' verlieben." Über B wie Berg folgt Walsers Exkursion in die Schrift der Ordnung des Alphabets Buchstabe für Buchstabe bis zum Ende: „X. hat nix. Über Y. komm' ich zu Z., einem Zeitungsbureau und gebe mein Alphabet ab." Diese emblematische Ausfaltung des Alphabets in seine Elemente und deren bildhafte Ausmalung - wohlgemerkt in einer Erzählung als Imagination einer buchstäblichen Evokation - findet ihre Pointe darin, dass die Bildererzählung des Alphabets am Ende tatsächlich einen zusammenhängenden Text ergibt, der über das „Zeitungsbureau“ als Text Walsers - als sein Alphabet - lesbar geworden ist. In der Welt seiner Bilder hat dieser Text also nichts anderes erzählt als die Geschichte seiner Genese aus den Buchstaben und der Welt, die sich mit ihnen und in ihrer Gestalt erzählen lässt.

9 Karl Philipp Moritz: Neues ABC-Buch. Illustrationen von Wolf Erlbruch. Nachwort von Heide Hollmer. München 2003, unpag. [S. 4].

10 Ebd. (Nachwort), unpag. [S. 59]. 
An diese Geschichte Walsers und die Figurationen seines Alphabets ließen sich systematische, historische und kulturvergleichende Beobachtungen anschließen, die sich um die Frage drehen, wie sich kulturelles Wissen in der Gestalt von Buchstaben oder Schriftzeichen ausprägt und wie diese konkreten Ausformungen in einer Dynamik kultureller Prozesse wirkmächtig werden können.

Die Geschichte des Buchstabens ist sehr lang. Wenn man auf die sinaitischen Vorläufer des phönizischen Alphabets zurückgeht, so ist die Schrift ca. 4000 Jahre, wenn man die alteuropäischen Zeichen der Vinča-Kultur und die 2003 in China entdeckten, auf Schildkrötenpanzern eingeschriebenen Zeichen als ,Buchstaben' versteht, dann (mit eingerechnet, dass, je weniger Kontext den Archäologen, Historikern und Kryptologen gegeben ist, die Definition als Schrift und nicht als Reihe abstrakter Einzelsymbole desto schwieriger sein muss) sogar ca. 7000 Jahre alt. Mit der Entstehung von Buchstaben bzw. Schrift unterscheiden die Historiker zwischen den prähistorischen Zeiten und dem Eintritt in die ,Geschichte'. Was bedeutet es, die ,Historie' mit der kulturellen Evolution des Buchstabens zusammenzudenken? Schon mit dieser Frage taucht die wesentlichere Frage auf, was überhaupt ein ,Buchstabe' sei. Wie wenig dieser Frage bislang Beachtung geschenkt wurde, zeigen aktuelle Publikationen zum Thema wie Max Bollwages Buchstabengeschichte(n) von 2010, das einmal mehr die Frage nach der Entstehung und - so der Untertitel - „warum unsere Buchstaben so aussehen“ nur in einem historischen Abriss der Formenentwicklung und des Zusammenhangs unterschiedlicher Buchstaben- bzw. Schreibstile behandelt. Auf die Buchstabengestalt bzw. -gestaltungen in ihrer Eigenbedeutung, ihrem Eigensinn und der Logik ihrer Sinnfälligkeit geht Bollwage nicht ein. Entsprechend nehmen ästhetische Perspektiven von der Kalligraphie über die Mythographie bis zur Mikrographie hier nur einen Randplatz ein..$^{11}$ In den Geschichten der Schriftentwicklung wird zudem meistens eine Linie von der Keilschrift zur griechischen und lateinischen Alphabetschrift gezogen, die eher in Nebenzügen andere Schriftsysteme - beispielsweise das älteste noch gebräuchliche Schriftsystem, wie es chinesische Kinder lernen - diskutiert. „A, B und C sind große Erfindungen - aber die Welt

11 Vgl. Max Bollwage: Buchstabengeschichte(n). Wie das Alphabet entstand - und warum unsere Buchstabgen so aussehen. Graz 2010. 
hört nach dem Buchstaben $Z$ noch längst nicht auf “, 12 könnte man eine Geschichte über Walsers „Alphabet“ hinaus anfangen lassen. In gewisser Weise kann man davon sprechen, dass die Digitalisierung nach Möglichkeit aller Schriftsätze der Welt, wie sie der weltweite Standard Unicode vorsieht, einen technologischen bzw. algorithmischen Rahmen bereitstellt, um Schriftzeichen vergleichend darzustellen und die Basis für eine „Typographische Semiotik“ zu schaffen. ${ }^{13}$

Der Buchstabe wurde in bisherigen Forschungen zumeist gemäß seiner Entwicklungsgeschichte, in seiner Funktion innerhalb eines jeweiligen Schriftsystems und einer grammatikalischen Ordnung, in seinem Charakter als Zeichen, Bild, Design, Form usw. semiologisch oder kulturhistorisch erforscht. Gegenüber diesen historisch-systematischen Kontextualisierungen sollen im Folgenden die einzelnen und konkreten Phänomene der Buchstäblichkeit als kulturelle Gebilde betrachtet werden. Schriftzeichen sind Ausformungen, deren Besonderheit darin besteht, lesbar zu sein. Während die Lesbarkeit den kognitiven gegenüber dem sinnlichen Aspekt hervorhebt, bleibt darauf zu insistieren, wie Buchstaben zunächst keine - und zwar kraft ihrer Phänomenalität keine - kognitiven Entitäten, sondern Figurationen sind. ${ }^{14}$ Der Prozess ihrer Gestaltgebung, ihre Einschreibung, geht nicht im aristotelischen Form/Stoff-Dualismus bzw. Hylemorphismus auf. Wie der aufschlussreiche Begriff der Spur verdeutlicht, der als figura etymologica zum Feld des Lesens, lat. legere, des Aufsammelns, oder auch der metaphorischen Diktion des Spurenlesens gehört, figuriert Schrift ein Nicht-Mehr für ein Noch-Nicht, gibt das Schreiben der Schrift die Form des Textes, den Lektüren im Zeichen eines Abwesenden mobilisieren. ${ }^{15}$ Ziel ist es damit, Buchstaben in der Genese ihrer Gestalt als Elemente der Kultur

12 Johannes Bergerhausen und Siri Poarangang: decodeunicode - die Schriftzeichen der Welt. Mainz 2011, S. 15.

13 Ebd., S. 13. Fragen der Ästhetik, das heißt der Wahrnehmung und der Welthaltigkeit von Buchstaben, sind damit nicht notwendigerweise mit behandelt.

14 Die Verwendung des Wortes ,Buchstaben' markiert in Anspielung auf den Titel des Bandes den Ausgangspunkt bei Alphabetschriften, schließt aber synekdochisch die Bedeutung ,Schriftzeichen“ (das genus proximum) mit ein.

15 Als literaturwissenschaftliche Binsenweisheit formuliert Klaus Weimar einen entsprechenden Aufsatztitel: Lesen: zu sich selbst sprechen in fremdem Namen. In: Heinrich Bosse, Ursula Renner (Hrsg.): Literaturwissenschaft - Einführung in ein Sprachspiel. Freiburg i. Br. 1999, S. 49-62. 
zu erkennen sowie ihre historische Wirkmächtigkeit für die kulturellen Bedeutungssysteme im Ausgang von ihrer konkreten Form zu analysieren. Allgemein gefasst lassen sich die kulturell wie historisch divergenten Ausprägungen von Buchstabengestalten - in verschiedenen Alphabeten wie etwa dem phönizischen, griechischen, lateinischen und in verschiedenen Schriftarten (Monumental- vs. Alltagsschrift, durch verschiedene Werkzeuge wie Feder oder Pinsel bestimmt etc.) - als Konkretisierungen und Manifestationen kultureller Gewohnheiten und epistemischer Grundlagen bestimmen; umgekehrt kann die Gestalt eines Buchstabens selbst wirkmächtig werden, wie beispielsweise die Form der römischen capitalis monumentalis, die Entwicklung von Initialen oder das Spiel mit der Schriftbildlichkeit. So lassen sich nicht nur Buchstaben als Teil kultureller Traditionen und Praktiken beschreiben, sondern, umgekehrt, Kulturen von ihren Buchstaben her beleuchten. Dies ermöglicht zugleich einen Kulturvergleich in nuce und im Ausgang von den figurativen Aspekten der Schriftzeichen. Es sollen also nicht nur einzelne Schriftzeichen verglichen werden, sondern zugleich sinnbildliche Bedeutungen in den verschiedenen Kulturen vergleichend fruchtbar gemacht werden.

Von der Einführung der Vokale und ihrer Grapheme ins griechische Alphabet über die Bedeutung des Buchstabens in der platonischen Sprachauffassung etwa im Kratylos bis zu den vermeintlich ,bildlicheren Schriftzeichen Ostasiens oder zahlenbasierten Computer-Sprachen bestimmt sich Sprachliches nicht zuletzt von den Schreibpraktiken und der visuellen Gestalt der Schriftzeichen her. Wie wirken sich Figurationen von Schriftzeichen auf das System einer jeweiligen Sprache aus?

Der Horizont dieser Fragen ist in den Traditionen unserer Denksysteme und begrifflichen Genealogien zumeist eher unterbestimmt geblieben; gleichwohl lassen sich für die terminologische Vagheit, mit der es Bestimmungen eines Wissens, das weder bloß empirisch sinnlich, noch rein spekulativ zu denken ist, zu tun haben, kategoriale Räume angeben. Was nun die Buchstaben und das Buchstäbliche betrifft, so gehört - ein Teil der begrifflichen Verwirrungen - seine Erörterung wesentlich in den Bereich der Rhetorik, für die das Buchstäbliche das Wörtliche sein kann, d. h. also im Innersten der Rhetorik eine Vorstellung des Eigentlichen, der Zusammengehörigkeit von res und verba lauert, die im Buchstaben ihre sei es nun Metonymie oder Metapher sieht, ihre amplificatio oder gar figura etymologica, wenn man in den Buchstaben, im Buchstäblichen, die Stäbe oder strichartigen Kerben mithört, mit denen Runen ins Holz eingeritzt wurden. Doch wie steht es um dieses Verhältnis der Sprache zur Wirklichkeit bzw. zu ihrer Wirklichkeit, für die die Buchstaben wörtlich einstehen? 
Die alte Metapher vom Buch der Natur, in dem man so lesen kann, wie man Buchstaben auf dem Papier aufsammelt, ist weitaus spannungsvoller, als es eine Rhetorik nahelegen könnte, die zwischen dem Eigentlichen und dem Uneigentlichen, dem Metaphorischen und dem Wörtlichen unterscheidet. Denn wie die Natur selbst avanciert das Buch mit seinen Deckeln zum Sinnbild der Ganzheit, indem es den enzyklopädischen Anspruch aufrechthält, den das Zeitalter der sogenannten Buchgelehrsamkeit verspricht. „Umso erstaunlicher“, schreibt Hans Blumenberg in Die Lesbarkeit der Welt,

wenn das Buch doch zur Metapher der Natur selbst werden konnte, seiner antipodischen Feindin, die zu derealisieren es bestimmt zu sein schien. Desto gewichtiger, desto zwingender müssen die Antriebe sein, die diese Verbindung von Buch und Natur hergestellt haben. Es sind vielleicht nur zwei: Einmal Konkurrenz mit dem einen Buch, mit seiner Autorität, seiner Ausschließlichkeit, seinem Bestehen auf Inspiration. Zum anderen Faszination durch die Macht, die das Buch in sich selbst dadurch aufbringt, daß es Herstellung von Totalität leistet. Die Kraft, Disparates, weit Auseinanderliegendes, Widerstrebendes, Fremdes und Vertrautes am Ende als Einheit zu begreifen oder zumindest als einheitlich begriffen vorzugeben, ist dem Buch, woran auch immer es sie exekutiert, wesentlich. [...]

Die Natur, einmal als Buch verbildlicht, soll eben diese Qualität eines Ganzen aus einem Wurf schon haben [...] - das Paradox eines Buches, das sich dagegen verwahrt, Leser zu haben. ${ }^{16}$

Blumenbergs Erstaunen darüber, dass sich Buch und Natur konkurrierend gegenüberstehen und doch zugleich metaphorisch füreinander einstehen können, beruht auf der Beobachtung, dass die Sprache des Buches und die Sprache der Natur, das menschliche Sprachsystem und die geschöpfte Natur nicht aus einer gemeinsamen Wurzel stammen, sondern erst aus der Distanz in eine Entsprechung gesetzt werden. Die Trennung von Sprache und Welt, verba und res, Buch und Natur bedingt ihren metaphorischen Bezug. Der Natur als Ergebnis göttlicher Schöpfung korrespondiert die Vorstellung einer Sprache als System, als Funktionszusammenhang, der entweder das (im romantischen Sinn) goldene Zeitalter hinter sich gelassen hat oder auf dem Weg zur Idealsprache oder

16 Hans Blumenberg: Die Lesbarkeit der Welt. Frankfurt a. M. 1981, S. 17 f. 
ihrer idealen Konstruktivität ist. Von den Ideen einer lingua universalis bis hin zu Chomskys Universalgrammatik dokumentiert sich dieser Glaube an ein aus sich heraus beschreibbares Sprachsystem. Blumenbergs Rückblick auf die rhetorische Feindschaft von Buch und Natur, der Welt und der Bücherwelt, der Sprache und dessen, worauf sie verweist, gehört damit einer Tradition an, in der „das Zeichen absolut willkührlich [erscheint]“, wie August Ferdinand Bernhardi in seiner Sprachlehre - ziemlich genau um die Jahrhundertwende 1800 - formulierte. ${ }^{17}$

Bernhardis erstaunlich klarer Satz, der bereits an Formulierungen der nach-saussureschen Linguistik anschließbar sein könnte, hat in seiner Kopplung von Absolutheit und Willkür für Reflexionsbedarf gesorgt. In der Nachfolge Bernhardis hat - und dies ist die hier eigentlich spannende Einlassung - der Sprachgelehrte, Orientalist und Dichter Friedrich Rückert versucht, diese denkbaren Konsequenzen aus der rhetorischen Optionalität des Sprach-Welt-Bezuges und des Arbitraritätskonzeptes auszuweiten. In seiner Idee der Philologie von 1811 insistiert auch Rückert auf einer Absolutheit des Sprachsystems, seiner intakten Ganzheit unabhängig von der Welt. Rückert sieht diese systemische Ganzheit im Anschluss an Fichtes nachkantische Wende zu identitätsphilosophischen Fragen in der Sekundarität der Sprache gegeben: Ihre Abbildungsfunktion für die Vernunft bedinge sie als „absoluta effigies“, so dass in einer Idealsprache aus der Ähnlichkeit von Sprache und Welt letztlich die Identität der Aussagemöglichkeiten mit der gegebenen Welt zu folgern wäre. Willkürlichkeit der Zeichen und abbildhafte Ähnlichkeit bestimmen sich von hier aus nicht als gegensätzliche Sprachkonzepte, sondern als vermittelbar. Denn einerseits ist auch für Rückert die Sprache ein sekundäres und darin willkürliches System, das aber in der gesteigerten Vorstellung andererseits gerade, indem es sich vollkommen ausbildet, mit der Idee der Welt selbst zusammenfällt. Das Abbildhafte negiert hier nicht die Willkür, und die Willkür bestimmt sich nicht als Defizit des Abbildhaften, sondern beides zusammen erhält einen Spannungsbegriff der ,lebendigen Sprache', d. h. des - im Singular - ,lebendigen Wortes' im Gegensatz zu den - im Plural - ,toten Wörtern' ${ }^{6}{ }^{18}$ - Bei Schelling wird hieraus ein systematischer Komplex, gemäß dem „Identitätsphilosphie“ heißt, „daß in jenem Ganzen Subjekt und Objekt mit gleicher Selbständigkeit einander

17 Friedrich Ferdinand Bernhardi: Sprachlehre. Berlin 1801-1803. Reprint Hildesheim und New York 1973. Bd. 1, S. 54.

18 Vgl. Stefan Willer: Poetik der Etymologie. Texturen sprachlichen Wissens in der Romantik. Berlin 2003, S. 174-181. 
gegenüberstehen, das eine nur das ins Objekt hinübergetretene [...], das andere nur das als solches gesetzte Subjekt sei“19.

Nun erlaubt es Rückerts in sich aporetisches Unternehmen, das in der Vermittlung von Differenz und Indifferenz, des Reellem mit dem Ideellen seinen eigentlichen Nexus des kultur- wie naturgeschichtlichen Lebens findet, dem Sekundären einen in sich konstitutiven Beitrag zuzusprechen. Und das gilt auch für den Buchstaben. Während Rückert in phonozentrischer Tradition den Laut als "fluens in tempore“, als ,Jetztzeit", als das in der Zeit Fließende, erläutert, kann er zugleich den Buchstaben als „consistens in spatio“ bestimmen: als Moment der Räumlichkeit bzw. des im Raum fest Stehenden. Für eine solche "forma duplex" der Sprache gelte, dass sowohl „der Laut die Idee selbst unmittelbar abbildet“, als, „daß auch der Buchstabe die Idee selbst unmittelbar abbildet.“ Denn „auch wir übertragen, wenn wir still lesen, die Buchstaben nicht in Laute, sondern wir transferieren die Buchstaben selbst, indem wir sie ansehen, in das Gemüt und nehmen sie gleichsam mit dem inneren Ohr des Geistes auf." ${ }^{\text {20 }}$

Ich möchte hieran nun zwei Überlegungen anschließen: Zunächst ist das Ziel der Idealsprache bei Rückert in doppelter Hinsicht positiv bestimmt: als vergangenes oder als zukünftiges, wobei der Unterschied zwischen dem verlorenen Vergangenen und dem zukünftig Unbestimmten interessanter Weise keine Rolle für Rückert spielt - ob „factam seu faciendam", ob "geschaffen oder noch zu schaffen“, bleibt in geradezu lakonischer Manier offen. ${ }^{21}$ Seine Wertungen hängen damit an einer romantisch-triadischen Geschichtsphilosophie, deren Umkehrseite man in Baudrillards Theorie der Simulakren beschrieben sehen kann. Denn ähnlich wie Rückerts „forma duplex“ lässt sich die Funktion von Simulakren erklären, die, je unabhängiger von der Welt sie diese berechnen, sie desto mehr mit der Welt übereinstimmen. Das digitale Spiel zwischen o und 1, zwischen on und off, Information und Nicht-Information erhöht die berechnende Erfassung der Welt, indem sie sich dieser immer

19 Friedrich Wilhelm Joseph Schelling: Werke. Nach der Original-Ausgabe in neuer Anordnung hrsg. von Manfred Schröter. München ${ }^{3} 1979$. Bd. 5: Schriften zur geschichtlichen Philosophie, S. $553 \mathrm{f}$.

20 Friedrich Rückert: Idee der Philologie (Dissertatio philologico-philosophica de idea philologiae), $\rrbracket_{3}-6$. Vgl. die Übersetzung der Dissertatio in: Claudia Wiener: Friedrich Rückerts „De idea philologiae“ als dichtungstheoretische Schrift und Lebensprogramm. Schweinfurt 1994, S. 173-285.

21 Vgl. Willer: Poetik der Etymologie (wie Anm. 18), S. 178. 
unähnlicher macht, indem an die Stelle der mannigfaltigen Sinnesdaten von der Welt eine genuin künstliche und der Welt indifferente Verarbeitungstechnologie tritt. Wenn die Welt mit genügend ,Speicherkapazität und ,höchster Auflösung' zu Ende berechenbar wäre, würde freilich mit diesem Phantasma die Frage auftauchen, wie sich das Simulakrum einer zu Ende berechneten Welt zur Idee des Endes der Welt verhielte. Rückerts Phantasma einer philologischen Idealsprache erhält in der Idee einer ,Auflösung' der Welt in ihrer Berechnung ihr technologisches Gegenstück.

Zweitens eröffnet Rückerts Philologie unabhängig von diesen Alternativen zwischen goldenem Zeitalter und Apokalypse der Zeichen einen systematischen Raum, um das Sekundäre der Sprache in ihr strukturelles Zentrum zu versetzen. Denn in der Sekundarität erfüllt sich ihr strukturell Absolutes.

Es erübrigt sich an dieser Stelle auf die Dekonstruktion des Gegensatzes von Primärem und Sekunärem in Jacques Derridas Grammatologie ausführlicher einzugehen. Von besonderem Interesse in diesem Zusammenhang ist jedoch eine Überlegung, die ich an Ryôsuke Ôhashis Bemerkungen zu Derridas Kritik am Phonozentrismus anschließen kann und die das Verhältnis des Bildes zur Schrift, der Schrift zum Bild betreffen. Im Anklang an Heideggers pleonastische Wendung, dass die Sprache spricht, hatte Ôhashi Auffassungen Derridas reformuliert, der „so weit geht zu sagen, dass die Sprache des Wortes von einer ,Blindheit' spricht, [und] so wäre das Durchdringen von Schrift und Bild in Ostasien auch bei ihm in eigentümlicher Weise mit gedacht." (S. 13) Wie kann die Überlegung, dass, wenn die Sprache spricht (Heidegger), sie von einer Blindheit geschlagen ist, also die Sprache blind spricht (Derrida), entfaltet werden? Interessant ist zunächst, dass die Sprache, indem sie blind spricht, das Bild gerade auszuschließen scheint; inwiefern aber kann dieser Ausschluss zugleich eine Zusammengehörigkeit von Schrift und Bild implizieren oder sogar ausdrücken?

In den Aufzeichnungen eines Blinden setzt Derrida mit der Beobachtung ein, dass die Malerei, indem sie Blinde zeigt, sie zugleich Schreibende zeigt. In seiner Lektüre dieses Komplexes, mit dem aus der Perspektive der Malerei Schrift und Blindheit zusammenzugehören scheinen, entfaltet er diese Blindheit dreifach: Zunächst fokussiert Derrida den

Moment der ursprünglichen Bahnung, wo die ziehend-zeichnende Macht des Zugs [puissance traçante du trait] wirkt, in dem Augenblick, wo die Spitze an der Spitze der Hand (des Leibes überhaupt) 
sich im Kontakt mit der Oberfläche vorwärtsbewegt, wird die Einschreibung des Einschreibbaren nicht gesehen..$^{22}$

Derridas Begriff der Spur grundiert diese Passage und erläutert Blindheit als das, was in der Spurenlese notwendigerweise abwesend ist, das heißt „die Einschreibung des Einschreibbaren“. Die puissance traçante du trait ${ }^{23}$ die von diesem Moment ausgeht, kennt jedoch selbst keine räumliche oder zeitliche Dimension: Sie ist ein Nicht-Mehr der Einschreibung oder Einzeichnung und ein Noch-Nicht der Spur. Die Macht des trait, im Deutschen nur schwer mit Zug, Einfall oder Strich wiederzugeben, ist somit zugleich eine Ohnmacht, ein Ent- oder Verzug. Dies ist eine zweite Kennzeichnung der Blindheit, die also als Blindheit des trait zu denken ist:

Er [le trait] erscheint - oder vielmehr: verschwindet - ohne Verzug. Ich nenne ihn den Entzug [retrait] oder die Eklipse, das differentielle Nichterscheinen des trait, des Zugs oder Strichs. [...] Die Teilbarkeit des trait unterbricht hier jede reine Identifizierung und bildet [...] unsere allgemeine Hypothek für jedes Denken der Zeichnung, die letztlich, d.h. an der Grenze, de jure unzugänglich bleibt. [...] Nichts gehört dem Strich oder Zug (an) [...]. Er verbindet nur, fügt nur zusammen, indem er trennt. ${ }^{24}$

Mit dieser puissance, durch Trennung zusammenzufügen, verhilft der trait zu einer wechselseitigen Erläuterung von Schrift und Bild. Denn indem das Bildmotiv der Blindheit, des Blinden im Bild - dem die Aufzeichnungen eines Blinden gewidmet sind -, den Schreiber par excellence zeigt, kann man die Blindheit als malerische Faktur der Szene der Schrift erkennen: Für das Bild entsteht die Schrift aus der Blindheit der Spur, das heißt aus der für die Spur konstitutiven Abwesenheit. Spurenlesen bedeutet von hier aus, das Abwesende vor dem Anwesen der Spur erkennen zu wollen. Derrida nennt dies - dritter Zug des trait - die

Rhetorik des Strichs oder Zugs. Denn ist nicht der Entzug der Linie, ihr Zurückgezogenwerden in dem Moment, da der trait gezogen

22 Derrida: Aufzeichnungen eines Blinden (wie Anm. 4), S. 49.

23 Ausführlicher habe ich Derridas Arbeiten zum trait dargelegt in: Martin Roussel: Matrikel. Zur Haltung des Schreibens in Robert Walsers Mikrographie. Frankfurt a. M. und Basel 2009, Kap. 2.3.

24 Derrida: Aufzeichnungen eines Blinden (wie Anm. 4), S. 57. 
wird, dasjenige, was die Rede zuläßt? Und es gleichzeitig verbietet, die Zeichnung von dem diskursiven Gemurmel zu trennen, dessen Zittern sie durchdringt und erstarren läßt [transit] ${ }^{25}$

Indem die Spur den Raum einer Abwesenheit eröffnet, gibt sie ihre eigene Räumlichkeit preis, das heißt lässt an ihrer Stelle die Rede zu. Diese Möglichkeit, dass die Spur zurücktritt, um der Rede ihren domaine zu überlassen, kann gemäß der puissance traçante $d u$ trait die Zeichnung niemals völlig verlassen, die an das „diskursive[ ] Gemurmel“ angeschlossen bleibt, das sie zugleich kraft der Repräsentation ausschließt.

Bereits in der Grammatologie hatte Derrida diese Korrespondenz von Bild und Schrift angedacht, als er den trait als "das Element der formalen Differenz dar[gestellt]" sah, "die die Inhalte (die farbliche oder lautliche Substanz) hervortreten läßt. “" ${ }^{26}$ Der reine Laut und die pure Farbe sind demnach die beiden Phantasmen, die in der puissance traçante du trait figurieren. Man wird diese puissance traçante in der Korrespondenz, die sie zwischen Bild und Schrift herstellt, auch als eine Gewalt sehen müssen, die "das diskontinuierliche Kontinuum von Bild und Schrift" (S. 10), das Ryôsuke Ôhashi herausgearbeitet hat, konstituiert. Als einen gesteigerten ästhetischen Ausdruck hiervon kann man auch das ,Ringen“ bei Sôtatsu und Kôetsu ansehen, wo „Bild und Schrift“, noch einmal in Ôhashis Worten, „danach [trachten], sich gegenseitig ihre Seinsweise zu entreißen. “ Dabei geht „die geraubte eigene Seinsweise [...] in die andere Seinsweise über und verändert diese." (S. 10) Das Ringen der Künste und die puissance traçante du trait sind also selbst korrespondierende Figuren einer ästhetischen Wissenschaft.

Dieser Figuration, Figurationen dieser Art gilt das Interesse der Beiträge dieses Bandes, wo der Buchstabe Figur wird und die Figur des Buchstabens die Schrift inszeniert. „Buchstaben der Welt - Welt der Buchstaben“ hieß ein Workshop, der im Januar 2011 am Internationalen Kolleg Morphomata in Köln stattfand. Die Idee hierzu kam von Ryôsuke Ôhashi, der seit Jahrzehnten ein Grenzgänger zwischen fernöstlichen und westlichen Kulturen ist und der nicht nur gelernt hat, die Denk- und Schriftsysteme zu wechseln, sondern der beharrlich die Idee reiner, gänzlich geschiedener

25 Ebd., S. 59.

26 Jacques Derrida: Grammatologie (wie Anm. 3), S. 358. 
und lediglich aufeinander zu beziehender Kulturräume kritisiert. Sein Anliegen hat er zur Eröffnung des Workshops in Worten skizziert, die man als noch früheres Motiv zu seiner hier vorangestellten Einleitung und retrospektiven Begründung verstehen kann und mit denen sich insofern ein Kreis schließt:

Die Frage nach der Denktradition ist tief verbunden mit der Frage nach der Sprache, und diese Frage wird wiederum verbunden mit der Frage nach den Buchstaben. Ich benutze z. B. die chinesischen Schriftzeichen und die japanische Lautschrift. Wenn ich auf Deutsch einen Aufsatz schreibe, frage ich mich immer, mit welcher Sprache ich gerade denke und schreibe. Wer mit chinesischen Schriftzeichen und japanischer Lautschrift denkt, so sage ich oft, denkt nicht diskursiv-waagerecht, sondern anschaulich-senkrecht. Aber ich muss ein senkrechtes Denken mit Alphabeten waagerecht abschreiben, und dies ist die Situation, in der ich mich seit meiner Promotion vor 35 Jahren immer wieder befinde, und die ich in diesem Kolleg erneut, in intensiviertem $M a ß$, erlebt habe. Inwieweit sind die Buchstaben für die jeweilige Sprache, Kultur und Denkweise konstitutiv? Das Thema ,Buchstaben' ist insofern mein eigenes Anliegen. Aber die Frage, inwieweit der Buchstabe als Morphom zu betrachten sei, ist das Anliegen des Kollegs Morphomata.

Sie sehen, dass das Thema des Workshops zweiteilig und jede Hälfte die Umkehrung der anderen Hälfte ist. Ich hatte als Mitorganisator das Thema so konzipiert, und zwar mit dem Erwartungshorizont, dass im Horizont der ,Buchstaben der Welt' eher linguistischsprachwissenschaftlich diskutiert wird, während, Welt der Buchstaben' dazu einlädt, das Thema philosophisch-phänomenologisch zu durchleuchten. Das Wort, Welt' in der ersten Hälfte ist eher im äußerlich-geographischen Sinne als Außenwelt zu verstehen. In dieser Außenwelt gibt es so mannigfaltige Buchstaben, deren Herkunft und Genese und Modi untersucht wurden und weiter untersucht werden sollen. Aber in der letzten Hälfte bedeutet der Begriff, Welt' eher eine Innenwelt, die den Buchstaben selbst immanent ist. Er kennzeichnet vor allem das phänomenologisch-philosophisch zu beleuchtende, innere Potential der Buchstaben.

Dem ist auch im Hinblick auf die - gegenüber dem Workshop erweiterten und ergänzten - Beiträge dieses Bandes nichts hinzuzufügen. Sie zielen angesichts des breiten Arbeitsfeldes weniger auf einen enzyklopädischen 
oder sammelnden Aspekt, sondern auf Fallstudien und kulturvergleichende Ansätze. Mit der Fokussierung auf die Gestalt von Schriftzeichen sollen die interkulturellen Vergleichshinsichten über das kulturelle Formeninventar - über die Konkretisierungen kultureller Praktiken - eröffnet werden. Mein herzlichster Dank gilt Ryôsuke Ôhashi.

\section{ABBILDUNGSVERZEICHNIS}

1 Tawaraja Sôtatsu und Hon'ami Kôetsu: Shiki-sôka-shitae-kokin-sĥu (Sammlung-der-Gedichte-von-einst-und-jetzt-mit-unterlegten-Bildernvon-Pflanzen-der-vier-Jahreszeiten), 17. Jahrhundert. Aus: Hiroshi Mizuo: Sôtsu und Kôrin. Hrsg. von Katsuchiro Kamai. Tokyo 1969 (Nihon no Bijutsu [Schöne Kunst in Japan]; 18), S. 116.

2 Albrecht Dürer: Selbstbildnis mit Eryngium (1493). Musée du Louvre, Paris.

3 Paul Klee: Anfang eines Gedichtes (1938). Zentrum Paul Klee, Bern.

4 Graffito. Foto Ryôsuke Ôhashi.

5 Vincent van Gogh: Blühender Pflaumenbaum (nach Hiroshige) (1887). Van Gogh Museum, Amsterdam. 



\section{WAS IST EIN BUCHSTABE?}



BEATRICE PRIMUS UND LUTZ WAGNER

\section{BUCHSTABENKOMPOSITION}

\section{GRUNDANNAHMEN}

Das wichtigste Motiv für die mehr als 5000 Jahre umfassende Schriftentwicklung war der Bedarf nach dauerhafter Speicherung von Wissen. Die Schrift führte jedoch nicht nur zu einer größeren Haltbarkeit von Wissensbeständen. Zugleich führte sie zu einer Fixierung der sonst flüchtig bleibenden Sprache und somit zu einer Abbildung der Sprache selbst, die nun erstmals als vergegenständlichtes Objekt zur Verfügung stand. Schriftsysteme haben im Zuge ihrer historischen Entwicklung die strukturellen Wesenszüge von Sprache im Allgemeinen und der jeweils repräsentierten Sprache im Besonderen angenommen. Der folgende Beitrag untersucht die Buchstabenstruktur mehrerer historisch tradierter Buchstabenschriften. In einem Buchstabenssystem, auch Alphabet genannt, entsprechen den Grundeinheiten des Schriftsystems die Grundeinheiten der zugehörigen Lautsprache, phonologische Merkmale und Phoneme, und nicht etwa Silben wie bei Silbenschriften. In Buchstabensystemen können daher prinzipiell alle weiteren Struktureinheiten der zugehörigen Lautsprache grafisch repräsentiert werden. Für sie gilt daher das oben Gesagte, hier in der Formulierung Wilhelm von Humboldts, im besonderen Maße: „Wodurch die Buchstabenschrift noch viel wesentlicher [...] auf die Sprache wirkt, ist dadurch, dass sie allein erst die Einsicht in die Gliederung derselben vollendet." ${ }^{\text {1 }}$

Historisch tradierte Buchstabenschriften, wie die moderne lateinische, griechische und arabische Schrift sowie die Tifinagh-Schrift der

1 Wilhelm von Humboldt: Über die Buchstabenschrift und ihren Zusammenhang mit dem Sprachbau. In: ders.: Werke in fünf Bänden. Bd. 3: Schriften zur Sprachphilosophie. Hrsg. von Andreas Flitner und Klaus Giel. Darmstadt ${ }^{8} 1996$ [1824], S. 82-112, hier S. 98. 
Hoggar-Tuareg, die im Folgenden näher betrachtet werden, blicken auf eine Jahrhunderte währende Entwicklung zurück, die sich weitgehend unabhängig vom normativen Diskurs und vom Eingriff einzelner Individuen oder Institutionen vollzieht. Entwicklungen dieser Art sind auch für andere sprachliche Bereiche gut belegt. In der Sprachgeschichte spricht man von ,Phänomenen der dritten Art', die wie von einer ,unsichtbaren Hand' gelenkt werden. ${ }^{2}$ Systemtheorien liefern einen angemessenen begrifflichen Rahmen für ihre Erklärung.

Unvorhergesehene Veränderungen, aber auch Ordnung und Selbstorganisation entstehen in allen Bereichen nach den Gesetzen komplexer dynamischer Systeme. Sie werden bereits erfolgreich in den Naturwissenschaften untersucht - von atomaren und molekularen Systemen in Physik und Chemie über zelluläre Organismen und ökologische Systeme der Biologie bis zu neuronalen Netzen der Gehirnforschung. In den Kulturwissenschaften ist Niklas Luhmanns Systemtheorie besonders einflussreich. Daher werden die hier relevanten systemtheoretischen Erkenntnisse anhand seiner Begrifflichkeit kurz vorgestellt. ${ }^{3}$

Ein selbstorganisierendes System besteht aus Elementen, die in ständiger Wechselwirkung zueinander stehen. Relationen bestimmen die Elemente und umgekehrt die Elemente die Relationen. Mit Bezug auf Buchstabenschriften bestimmen die Relationen zwischen den Buchstabenteilen die Buchstaben und umgekehrt die Buchstaben die Relationen zwischen ihren Teilen. Nach Luhmann kann eine Ausdifferenzierung von Systemen nur durch Selbstreferenz zustande kommen. Ein System ist selbstreferentiell, wenn es die Elemente, aus denen es besteht, als Funktionseinheiten selbst konstituiert. ${ }^{4}$ Buchstaben und deren Komponenten referieren stets auf sich selbst als Zeichen und konstituieren sich selbst als Elemente des Schriftsystems. Systeme

2 Vgl. Rudi Keller: Sprachwandel. Von der unsichtbaren Hand in der Sprache. Tübingen ${ }^{2} 1994$.

3 Vgl. Niklas Luhmann: Soziale Systeme. Grundriß einer allgemeinen Theorie. Frankfurt a. M. 1984; ders.: Einführung in die Systemtheorie. Heidelberg 2004.

4 Luhmann: Soziale Systeme (wie Anm.3), S. 59. Man kann mit Ludwig Jäger auch sagen: Die Elemente eines selbstreferentiellen Systems haben Eigen-Sinn. Vgl. Ludwig Jäger: Vom Eigensinn des Mediums Sprache. In: Dietrich Busse, Thomas Niehr, Martin Wengeler (Hrsg.): Brisante Semantik. Neuere Konzepte und Forschungsergebnisse einer kulturwissenschaftlichen Semantik. Tübingen 2005, S. 45-64. 
müssen, um selbstreferentiell zu sein, mindestens die Differenz von System und Umwelt systemintern als Orientierung und als Prinzip der Erzeugung von Informationen verwenden können. Selbstreferentielle Geschlossenheit ist daher nur in einer Umwelt möglich. ${ }^{5}$ Eine wechselseitige Beziehung mit der Umwelt, z. B. mit anderen Systemen, in unserem Fall dem Lautsystem der betreffenden Sprache, besteht weiterhin. Mit Bezug auf Buchstaben stellen wir zusammenfassend fest, dass sie nicht nur auf lautlich realisierte Zeichen referieren, sondern auch auf sich selbst als grafisch realisierte Zeichen. In selbstorganisierenden Systemen entstehen Strukturen, die nicht durch einzelne Systemelemente gesteuert sind, aber durch die Wechselwirkung von Elementen bei geeigneten Bedingungen möglich werden. Man spricht dann auch von Emergenz von Ordnung. So reproduzieren in der Chemie autokatalytische Stoffe sich selbst und erzeugen bei geeignetem Stoff- und Energieaustausch organische Lebensfunktionen. Solchen Systemen spricht Luhmann Handlungsfähigkeit zu. ${ }^{6}$ Aus dieser systemtheoretischen Perspektive lassen sich die relative Geschlossenheit der hier untersuchten Buchstabensysteme, konvergierende Entwicklungsschritte und emergente, im normativen Diskurs nicht erkannte Ordnungs- und Gliederungsprinzipien erklären. Der Gebrauch solcher Systeme basiert auf einem impliziten, nicht-artikulierbaren, reflexartigen Wissen, einem Wissenstyp, der auch dem Sprachgebrauch zugrunde liegt.

Ein wichtiges emergentes Prinzip, das im Fokus des vorliegenden Beitrags steht, ist die Kompositionalität der Buchstabenformen. Das Kompositionalitätsprinzip ist das wichtigste Prinzip der Semantik und geht auf Gottlob Frege zurück, weshalb es auch als Frege-Prinzip bekannt ist. ${ }^{7}$ Es lautet: Die Bedeutung eines komplexen Ausdrucks wird von der

5 Luhmann: Soziale Systeme (wie Anm. 3), S. 25.

6 Ebd., S. 229. Mit dem Luhmann'schen Handlungsbegriff verwandt ist der von Colin Pittendrigh eingeführte Begriff der Teleonomie. Vgl. Colin S. Pittendrigh: Adaptation, Natural Selection, and Behavior. In: Anne Roe, George Gaylord Simpson (Hrsg.): Behavior and Evolution. New Haven 1958, S. 390-416. - Als teleonomisch bezeichnet Pittendrigh Prozesse, wenn sie aus ihren Komponenten und Strukturen selbst erklärt werden. Sie bedürfen dadurch keiner Zusatzannahmen über mögliche externe teleologische oder intentionale Einflüsse. Wir danken Ludwig Jäger für diesen Hinweis.

7 Gottlob Frege: Über Sinn und Bedeutung. In: Zeitschrift für Philosophie und philosophische Kritik (1892), NF 100, S. 25-50. 
Bedeutung seiner Teile und der Art ihrer Verknüpfung determiniert. Inzwischen ist Kompositionalität als weit umspannendes Prinzip anerkannt. So entdeckt Tim Horton ${ }^{8}$ Kompositionalität in der tonalen Struktur von Musik, während Ray Jackendoff und Zenon Pylyshyn mit Bezug auf die visuelle Objektwahrnehmung davon ausgehen, dass komplexe visuelle Formen anhand elementarer Teile und ihrer Anordnung verarbeitet werden. ${ }^{9}$ Kompositionalität ist eine zentrale artspezifische Fähigkeit menschlicher Sprache und menschlichen Denkens. ${ }^{10}$

Das Kompositionalitätsprinzip operiert syntagmatisch; es garantiert die funktionale Transparenz kopräsenter, miteinander verknüpfter Elemente. Auf paradigmatischer Ebene gewährleistet das Prinzip des konstruktionellen Ikonismus die funktionale Transparenz füreinander substituierbarer Elemente. Dem Prinzip des konstruktionellen Ikonismus zufolge entspricht einer einfacheren (linguistisch betrachtet weniger markierten) Form eine einfachere (weniger markierte) Funktion und einer komplexeren (markierteren) Form eine komplexere Funktion. ${ }^{11}$

Kompositionalität und konstruktioneller Ikonismus garantieren funktionale Transparenz in Symbolsystemen, zu denen menschliche Sprachen und Buchstabenschriften gehören. Bei einem Symbol stehen Form und Funktion grundsätzlich in einem arbiträren, nicht unmittelbar transparenten Verhältnis zueinander. In Terence Deacons semiotischem Evolutionsszenario, das bereits bei Charles Peirce angelegt

8 Tim Horton: The Compositionality of Tonal Structures: A Generative Approach to the Notion of Musical Meaning. In: Musicæ Scientiæ 5 (2001), H. 2, S. 131-156.

9 Ray Jackendoff: On Beyond Zebra. The Relation of Linguistic and Visual Information. In: Cognition 26 (1987), S. 89-114; Zenon W. Pylyshyn: Some Primitive Mechanisms of Spatial Attention. In: Cognition 50 (1994), S. 363-384.

10 Michael A. Arbib, Derek Bickerton (Hrsg.): The Emergence of Protolanguage. Holophrasis vs Compositionality. Amsterdam 2010; Derek Bickerton: Language and Species. Chicago 1990; Henry Brighton, Simon Kirby: The Survival of the Smallest: Stability Conditions for the Cultural Evolution of Compositional Language. In: Jozef Kelemen, Petr Sosík (Hrsg.): Proceedings of the $6^{\text {th }}$ European Conference on Artificial Life. Dordrecht 2001, S. 592-601; Elizabeth Spelke: What Makes Us Smart? Core Knowledge and Natural Language. In: Dedre Gentner, Susan GoldinMeadow (Hrsg.): Language in Mind. Cambridge 2003, S. 277-311.

11 Willi Mayerthaler: Ikonismus in der Morphologie. In: Zeitschrift für Semiotik 2 (1980), S. 19-37. 
ist, ${ }^{12}$ entwickeln Kommunikationssysteme, welche die ursprüngliche piktografische (im semiotischen Sinn ikonische) Phase überwunden und die höchste semiotische Entwicklungsstufe des Symbolischen erreicht haben, zwangsläufig Selbstreferentialität und prinzipiengeleitete Kombinatorik, wobei letztere die Grundlage für Kompositionalität bildet. ${ }^{13}$ In den Worten Deacons:

Because symbols do not directly refer to things in the world, but indirectly refer to them by virtue of referring to other symbols, they are implicitly combinatorial entities whose referential powers are derived by virtue of occupying determinate positions in an organized system of other symbols. Both their initial acquisition and their later use requires a combinatorial analysis. ${ }^{14}$

In diesem Beitrag werden wir zeigen, dass historisch tradierte Buchstabensysteme kompositionell und konstruktionell ikonisch sind. Die Buchstaben solcher Systeme bestehen aus wenigen Grundelementen, die durch eine regelgeleitete Kombination komplexere Einheiten bilden. Die Form der Einheiten und die Art und Weise ihrer Verknüpfung determinieren ihre sprachliche Funktion. Die wichtigste sprachliche Funktion von Buchstaben ist die Repräsentation der Lautstruktur der zugehörigen Sprache und steht im Zentrum unserer Überlegungen. Diese Funktion wird im phonografischen Prinzip der Buchstaben- bzw. Alphabetschriften verankert. Wir werden zeigen, dass Buchstabenteilen und -merkmalen Lautmerkmale entsprechen. Damit entwickeln wir eine merkmalsbasierte, kompositionelle Version des phonografischen Prinzips, das in seiner traditionellen Ausprägung Buchstaben und Laute (Phone bzw. Phoneme) als Ganzheiten zueinander in Beziehung setzt.

Die hier vertretene Sicht auf Schrift- und Buchstabensysteme ist relativ neu. In der traditionellen Sichtweise ist Schrift eine individuelle

12 Terrence Deacon: The Symbolic Species. The Co-evolution of Language and the Brain. New York, London 1997; Charles S. Peirce: What is a Sign? In: ders.: The Essential Peirce: Selected Philosophical Writings. Bd. II.: (1893-1913). Hrsg. von The Peirce Edition Project. Bloomington 1998, S. 4-10.

13 Derek Bickerton: Symbol and Structure: A Comprehensive Framework for Language Evolution. In: Morten H. Christiansen, Simon Kirby (Hrsg.): Language Evolution. Oxford 2003, S. 77-93.

14 Deacon: The Symbolic Species (wie Anm. 12), S. 99. 
Erfindung, wie die folgenden Ausführungen von Günther Klaffenbach über die Entstehung der griechischen Schrift belegen: ,Wie der griechische Genius aus allem, was er von anderen übernommen hat, in einzigartiger Schöpferkraft ein Neues schuf, so hat er es auch bei der Übernahme der phönikischen Schrift getan." Und weiter heißt es über die griechischen Schriftzeichen: „Da nun diese Zeichen allen Sonderarten, in die sich das griechische Alphabet aufgespalten hat, gemeinsam sind, kann es sich nur um die bahnbrechende Tat eines Mannes handeln. " ${ }^{15}$ Diese Auffassung über Schrift und Schriftentwicklung hat dazu geführt, dass lange Zeit punktuelle Umbrüche anstelle langsam emergenter Eigenschaften, normative Aussagen über die Schrift anstelle des Schriftsystems selbst, die Monumentalschrift der kulturellen Elite anstelle der Gebrauchschrift des Alltags in den Fokus genommen wurden.

Die neuere, systemtheoretisch geprägte Auffassung über die Genese und Dynamik komplexer kultureller Systeme im Allgemeinen und der Schrift im Besonderen setzt sich allerdings immer mehr durch. So schreibt William Watt, der hinsichtlich der kompositionalen Struktur der Buchstaben Pionierarbeit geleistet hat:

The letters of the ordinary English alphabet, some of which date back to Egyptian forms of five millennia ago [...], belong to a coherent set of signifying elements that have mostly been shaped gradually through changes introduced inadvertently as part of their casual transmission from generation to generation. In short, they have been shaped, in large degree, by processes comparable to those which shape language itself..$^{16}$

Im Folgenden werden wir Kompositionalität und konstruktionellen Ikonismus in den modernen lateinischen Buchstaben (Abschnitt 2), in der modernen arabischen Schrift (Abschnitt 3) sowie in der TifinaghSchrift der Hoggar-Tuareg (Abschnitt 4) nachweisen. Wir werden auch ein gemeinsames Strukturmerkmal lateinischer und griechischer Buchstaben aufdecken. Diese Buchstabensysteme haben sich natürlich in einer Sprachgemeinschaft entwickelt und wurden nicht von einer Person oder Institution konzipiert und verordnet. Um den Unterschied

15 Günther Klaffenbach: Griechische Epigraphik. Göttingen 1957, S. 32 f. Kursivierung im Original.

16 William C. Watt: What Is the Proper Characterization of the Alphabet? In: Semiotica 70 (1988), S. 199-241, hier S. 199. 
zwischen historisch tradierten und künstlich erschaffenen Systemen zu illustrieren, werden wir im zweiten Abschnitt auf das koreanische Hangul kurz eingehen. Um eine komparatistisch angemessene Datenbasis zu garantieren, haben wir neben dem lateinischen Alphabet das moderne arabische und das Tifinagh-Alphabet gewählt. Die Buchstabenformen der beiden letztgenannten Alphabete unterscheiden sich deutlich von den Buchstabenformen des lateinischen Alphabets und beziehen sich auf Sprachen, deren Verwandtschaftsgrad mit mitteleuropäischen Sprachen und untereinander gering ist.

\section{DAS MODERNE LATEINISCHE ALPHABET}

Die ältesten Vorgänger des modernen lateinischen Alphabets wurden im 2. Jahrtausend vor Chr. im Nahen Osten von den Phöniziern und anderen altsemitischen Völkern entwickelt. Auf der phönizischen Schrift basieren nach einigen Wandlungen die Alphabete der griechischen, etruskischen und römischen Kultur. Auch das kyrillische Alphabet gehört zu dieser Schriftfamilie. Andere Entwicklungszweige führen uns zur hebräischen, arabischen und indischen Schrift. Das lateinische Alphabet besitzt noch heute mit wenigen Änderungen Gültigkeit für uns und hat sich fast weltweit verbreitet. Die lange Entwicklung und große areale Verbreitung des lateinischen Alphabets weisen auf ein hohes $\mathrm{Ma} ß$ an prinzipiengeleiteter Selbstorganisation, um die es uns hier geht, hin.

Das Kerninventar des lateinischen Alphabets ist in (1) aufgelistet:

(1) Majuskeln und Minuskeln in einer serifenlosen Druckschrift (Arial)

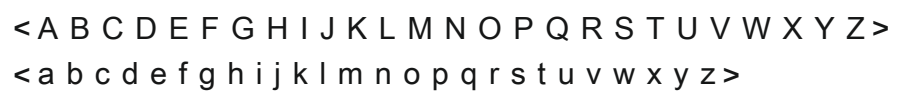

$\mathrm{Zu}$ diesen 26 Buchstaben kommen sprachspezifische Ergänzungen hinzu, wie z. B. der Buchstabe $<\beta>$ im Deutschen. ${ }^{17}$ Diakritische Zeichen - Punkte und Striche unter oder über einem Buchstaben - lassen wir für das lateinische Alphabet unberücksichtigt.

17 In diesem Beitrag folgen wir linguistischen Konventionen und notieren im Fließtext Buchstaben zwischen spitzen Klammern und Laute (Phoneme) zwischen schrägen Klammern. In Tabellen und Synopsen verzichten wir in klaren Fällen auf diese Notation. 
Das erste große Problem, das bei einer Merkmalanalyse auftaucht, ist die Buchstabenvariation. Es gibt Minuskeln versus Majuskeln und des Weiteren sehr viele Varianten dieser Varianten: verschiedene Druckversionen, normierte Handschriften und individuell schwankende Handschriften. Das Variationsproblem veranlasste Schriftsystemforscher ${ }^{18}$ eine Merkmalanalyse unseres Alphabets von vorneherein zu verwerfen. Dieser Schluss erscheint uns mit Blick auf die Phonologie voreilig. Denn auch Laute variieren erheblich in Abhängigkeit von benachbarten Lauten, vom Sprecher und vom Sprechtempo, um nur einige Faktoren zu nennen. Eine tragfähige Lösung des Variationsproblems wurde in der Phonologie mit der Unterscheidung zwischen konkretem Phon bzw. Allophon und abstraktem Phonem sowie mit der Unterscheidung zwischen phonetischen und bedeutungsunterscheidenden, phonologischen, Merkmalen bereitgestellt. Wir werden daher nur die sprachlich funktionalen Buchstabenmerkmale betrachten und sprachlich nicht-funktionale Merkmale wie etwa Serifen und Neigungsgrad außen vor lassen. Wir behandeln die Minuskeln als Grundvarianten. ${ }^{19}$ Dies lässt sich unter anderem damit begründen, dass Minuskeln im Normalfall und Majuskeln nur unter besonderen Bedingungen gebraucht werden.

Im Gegensatz zu Lauten, deren Merkmale gebündelt auftreten, sind Buchstaben syntagmatisch komplexe Gebilde, die aus vertikal oder horizontal angeordneten Komponenten bestehen. Syntagmatisch komplexe sprachliche Gebilde zeichnen sich dadurch aus, dass ein Element den Kopf oder Kern der Konstruktion bildet, von dem - falls vorhanden weitere Elemente, die wir für Buchstaben Codas nennen, abhängen. Die Unterscheidung zwischen Kopf und abhängigem Element ist für die Systematik der lateinischen Buchstaben von zentraler Bedeutung. Die meisten Regularitäten beziehen sich explizit entweder auf den Kopf oder die Coda eines Buchstabens oder auf die Art und Weise ihrer Verknüpfung. (2) illustriert die Kopf-Coda-Asymmetrie in der Komposition einer Minuskel und nennt die wichtigsten Unterscheidungskriterien:

18 Z. B. Manfred Kohrt: Problemgeschichte des Graphembegriffs und des frühen Phonembegriffs. Tübingen 1985, S. 441.

19 Herbert Brekle: Die Antiqualinie von ca. $-\mathbf{1 5 0 0}$ bis ca. +1500 . Untersuchungen zur Morphogenese des westlichen Alphabets auf kognitivistischer Basis. Münster 1999; Hartmut Günther: Schriftliche Sprache: Strukturen geschriebener Wörter und ihre Verarbeitung beim Lesen. Tübingen 1988. 
(2) Kopf-Coda-Unterscheidung am Beispiel des $<$ p $>$
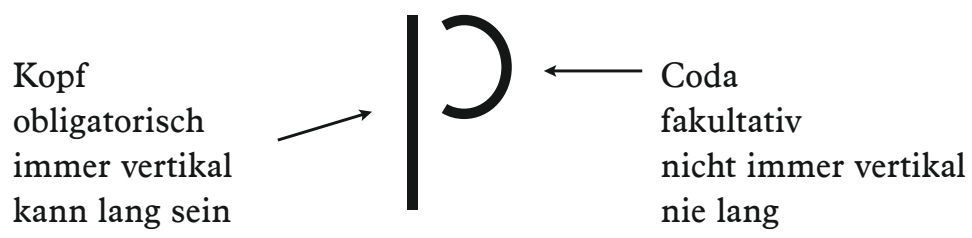

Ein erstes wichtiges Identifikationskriterium für Buchstabenköpfe folgt aus dem allgemeinen Prinzip, demzufolge der Kopf einer Konstruktion obligatorisch ist. Besteht ein Buchstabenkörper, wie etwa $<\mathrm{i}>$, lediglich aus einer Komponente, so ist diese Kopf. Des Weiteren sind Köpfe im lateinischen Minuskelsystem im Gegensatz zu Codas immer vertikal. Codas sind immer kurz (nie lang), während Köpfe, wie bei $<\mathrm{p}>$, lang sein können.

Wir illustrieren die Merkmale der modernen lateinischen Buchstaben anhand des Schriftsystems des Deutschen. Die Analyse lässt sich weitgehend auf andere phonografisch transparente Schriftsysteme wie Italienisch, Polnisch oder Ungarisch und weniger zuverlässig auf phonologisch opake Systeme wie Englisch übertragen. Die Analyse richtet sich am kanonischen Lautwert eines Buchstabens aus, wie er in Sprachhandbüchern des Deutschen angegeben wird.

Nachdem wir diese Vorüberlegungen getroffen haben, können wir uns nun den sprachlich relevanten Buchstabenmerkmalen zuwenden. ${ }^{20}$ Eine bestechende Eigenschaft unserer Minuskeln ist, dass einige einen langen Kopf entwickelt haben. Ein Buchstabenkopf hat das Merkmal [lang] genau dann, wenn er sich vertikal über das Mittelband und mindestens ein äußeres Band erstreckt oder im Mittelspatium schräg ist. ${ }^{21}$

20 Eingehender Beatrice Primus: A Featural Analysis of the Modern Roman Alphabet. In: Written Language and Literacy 7 (2004), H. 2, S. 235-274; dies.: Buchstabenkomponenten und ihre Grammatik. In: Ursula Bredel, Hartmut Günther (Hrsg.): Orthographietheorie und Rechtschreibunterricht. Tübingen 2006, S. 5-43; dies.: Die Buchstaben unseres Alphabets: Form - Entwicklung - Funktion. In: Dieter Boschung, Hansgerd Hellenkämper (Hrsg.): Kosmos der Zeichen - Schriftbild und Bildformel in Antike und Mittelalter. Wiesbaden 2007, S. 45-65.

21 Bei Primus: A Featural Analysis (wie Anm. 20) und dies.: Buchstabenkomponenten (wie Anm. 20) erscheint anstelle von [lang] das Merkmal [frei] und anstelle von [kurz] das Merkmal [nicht frei], d.h. die Absenz 
Sonst klassifizieren wir die Kopflinie als [kurz]. Die vertikale Ausdehnung unserer Minuskeln wird durch fünf virtuelle Linien und dementsprechend vier Bänder strukturiert, wobei die inneren Bänder zu einem in (3) schattierten Mittelband zusammenfallen:

(3) Die unterschiedliche vertikale Ausdehnung verschiedener Minuskeln

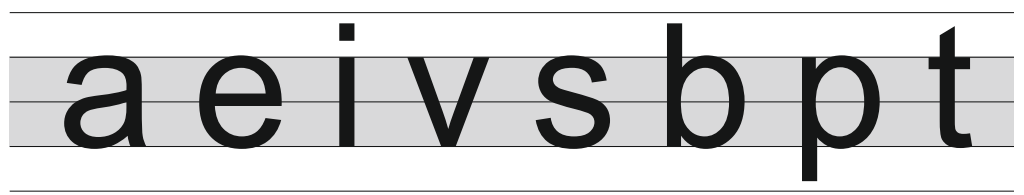

Der Längenkontrast visualisiert die lautliche Silbenstruktur in einem sehr zuverlässigen Ausmaß. Fünfzehn der siebzehn Buchstaben mit langer Kopflinie sind Lauten mit geringer Schallfülle vorbehalten, die keinen Silbenkern bilden können. Acht der neun Buchstaben mit kurzer Kopflinie entsprechen Lauten mit großer Schallfülle, die den Silbenkern bilden können. Vgl. (4):

(4) Buchstabenlänge und Silbenstruktur im modernen lateinischen Alphabet

\begin{tabular}{|c|c|c|}
\hline & transparent & opak \\
\hline $\begin{array}{l}\text { kurze Kopflinie } \\
\text { für silbenkernfähige Laute }\end{array}$ & $<\mathrm{i}$ u e o a $\mathrm{mn} r\rangle$ & $\begin{array}{l}<1> \\
<y>\text { in }<\text { lyrik }>\end{array}$ \\
\hline $\begin{array}{l}\text { lange Kopflinie } \\
\text { für nicht-silbenkernfähige } \\
\text { Laute }\end{array}$ & $\begin{array}{l}<p t k b d g h f j q> \\
<s \vee w z x> \\
<y>\text { in }<\text { yoga }>\end{array}$ & $<c>$ \\
\hline
\end{tabular}

Die in (4) als opak angegebenen drei Buchstaben lassen sich im Rahmen einer eingehenderen Analyse, die hier nicht präsentiert werden kann, gut erklären. ${ }^{22}$ So wird bspw. $<\mathrm{y}>$ nur für Fremdwörter verwendet, und zwar

des Merkmals [frei]. Eine ausgefeiltere Längenhierarchie, die wir hier übernehmen, weist auch die Schräge als Länge aus. Vgl. Nanna Fuhrhop und Franziska Buchmann: Die Längenhierarchie: Zum Bau der graphematischen Silbe. In: Linguistische Berichte 218 (2009), S. 127-155.

22 Vgl. Fuhrhop und Buchmann: Die Längenhierarchie (wie Anm. 21). 
für Laute mit widersprüchlichen Eigenschaften. Als silbenkernfähiges Vokalzeichen wie in $<$ lyrik $>$ ist $<y>$ opak; als Konsonantenzeichen wie in $<$ yoga $>$ ist der Buchstabe transparent.

Geht man davon aus, dass zwei benachbarte kurze Buchstaben in der Regel einem Silbenkern entsprechen, vgl. den ersten der beiden unterstrichenen Silbenkerne in der Aussprache /li $\underline{b} \underline{\underline{n}} /$ von lieben, so ist die Silbenstruktur der Wörter in unserer Alphabetschrift sehr zuverlässig angezeigt. Vgl. ein Beispiel in (5), in welchem jede Silbe durch genau einen langen Buchstabenkopf angezeigt ist (die initiale Majuskel von Brü-der erfüllt andere Funktionen, weshalb sie hier nicht aufgenommen wird):

(5) die lie-ben gu-ten brü-der

Der Längenkontrast visualisiert die lautliche Silbenstruktur auf eine sehr zuverlässige Weise auch in der modernen griechischen Minuskelschrift. Vgl. (6):

(6) Buchstabenlänge und Silbenstruktur im modernen griechischen Alphabet

\begin{tabular}{lll} 
& transparent & opak \\
\hline $\begin{array}{l}\text { kurze Kopflinie } \\
\text { für Silbenkern-Laute }\end{array}$ & $<\alpha \varepsilon$ । $\circ \cup \omega>$ & $<\eta>$
\end{tabular}

lange Kopflinie

$<\beta$ < $\delta$

für Silbenrand-Laute

Bsp. ,Philologe

$\varphi 1-\lambda 0-\lambda 0-\gamma \circ \varsigma$

Wir halten fest, dass in beiden Minuskelschriften die lautliche Silbengliederung auf recht zuverlässige Weise als regelgeleitete Abfolge von langen und kurzen Buchstabenköpfen transparent gemacht wird.

Die Artikulationsart, wie etwa die Unterscheidung zwischen Verschlusslauten (Plosiven), Reibelauten (Frikativen) und Sonoranten (Nasalen und Liquiden), ist für die Silbengliederung relevant und wird im modernen lateinischen Alphabet dementsprechend zusammen mit der Silbengliederung durch die Länge der Buchstabenköpfe repräsentiert. Verschlusslauten entsprechen in der Regel Buchstaben mit einem langen 
geraden (senkrechten) Kopf: $\langle\mathrm{p}, \mathrm{t}, \mathrm{k}, \mathrm{b}, \mathrm{d}\rangle$. Reibelauten entsprechen in der Regel Buchstaben mit einem nicht-geraden langen Kopf: $\langle\mathrm{f}, \mathrm{v}, \mathrm{w}, \mathrm{s}\rangle$. Sonoranten werden in der Regel durch kurze Buchstabenköpfe angezeigt: $<\mathrm{m}, \mathrm{n}, \mathrm{r}>$. Die - ggf. wie beim gerollten $/ \mathrm{r} /$ intermittierende - Schließung der Artikulatoren, die bei Konsonanten maximal und bei Vokalen aufgrund ihrer inhärenten Schallfülle weniger ausgeprägt ist, korrespondiert mit einer geraden vs. nicht-geraden Kopflinie. Vgl. (7):

(7) Artikulationsart im modernen lateinischen Alphabet am Beispiel des Deutschen

\begin{tabular}{lll} 
& transparent & opak \\
\hline $\begin{array}{l}\text { Gerade-senkrechte } \\
\text { Kopflinie }\end{array}$ & $<$ i u p b t d k q m n $\mathrm{r}>$ & $<\mathrm{g}>(<\mathrm{c}>)$ \\
für geschlossene & $<\mathrm{y}>$ in $<$ lyrik $>$ & \\
Artikulatoren & & \\
\hline nicht gerade-senkrechte $<$ e o a f v w s j $>$ & $<\mathrm{h}>(<\mathrm{x}>)$ \\
Kopflinie & \\
für nicht-geschlossene & $<\mathrm{y}>$ in $<$ yoga $>$ & \\
Artikulatoren &
\end{tabular}

In (7) erscheinen $<c>$ und $<x>$ in Klammern, weil diesen Buchstaben mit /ts/ bzw. /ks/ jeweils eine Lautverbindung mit widersprüchlichen Artikulationsarten entspricht, so dass sie partiell transparent sind. So gibt $<\mathrm{c}>$ bspw. den vorderen Laut /s/ in /ts/ transparent wieder.

Ein in mehreren Buchstabensystemen konstruktionell ikonisch repräsentierter Lautkontrast betrifft den Artikulationsort. Am zuverlässigsten kodiert wird der Kontrast zwischen vorderer und hinterer Artikulation, wobei die Grenze dieses Kontrasts sprach- bzw. schriftsystemspezifisch ausfällt. Für die hier untersuchten Schriftsysteme scheint der harte Gaumen (das Palatum) die Grenze zu markieren. Präpalatale Artikulation gilt für diese Schriftsysteme als vordere Artikulation, palatale und postpalatale als hintere Artikulation. Vordere Artikulation ist rein phonologisch betrachtet einfacher und besser wahrnehmbar als hintere Artikulation. Eine konstruktionell ikonische visuelle Repräsentation wird daher vordere Laute durch einfache, kanonische Buchstabenformen und hintere Laute durch komplexe, nicht-kanonische Buchstabenformen repräsentieren. Die Begründung ist einfach: Dem 
Prinzip des konstruktionellen Ikonismus zufolge korrespondiert eine einfache Form mit einer einfachen Funktion und dementsprechend eine komplexe Form mit einer komplexen Funktion. Was eine einfache oder komplexe Buchstabenform ist, gilt nur mit Bezug auf das jeweilige Buchstabensystem. Deswegen werden wir im Folgenden von kanonischen, d. h. systemkonformen, und von nicht-kanonischen, d. h. im System auffälligen, Buchstabenformen sprechen.

Die Ausrichtung kanonischer Buchstabenformen stimmt mit der Ausrichtung der Zeile überein. In modernen lateinischen Alphabetschriften ist die Zeile rechtsgerichtet und folglich sind nach rechts oder nicht-gerichtete Buchstaben kanonisch, linksgerichtete Buchstaben nichtkanonisch. Rechtsgerichtet ist ein Buchstabe, wenn sich seine gerundete Kopflinie nach rechts öffnet, wie etwa bei $\langle\mathrm{e}\rangle$ und $\langle\mathrm{f}\rangle$, oder wenn die Coda auf der rechten Seite des Kopfes liegt, wie etwa bei $\langle$ b $>$ und $\langle$ p $>$. Linksgerichtet ist ein Buchstabe, wenn sich seine gerundete Kopflinie nach links öffnet, wie z. B. bei $<j>$, oder wenn die Coda auf der linken Seite des Kopfes liegt, wie z. B. bei der Arialform von $\langle$ g $>$ sowie bei $<\mathrm{u}>$ und $<$ a $>$. Des Weiteren zeichnet sich eine kanonische Buchstabenform dadurch aus, dass sich Kopf und Coda einander zuwenden und damit eine möglichst geschlossene Figur bilden, wie bei $<$ b $>$ und $<$ p $>$ im Gegensatz $\mathrm{zu}<\mathrm{k}>$ und $<\mathrm{x}>$. Der konstruktionelle Artikulationsort-Ikonismus wird in (8) zusammengefasst:

(8) Artikulationsort im modernen lateinischen Alphabet am Beispiel des Deutschen

\begin{tabular}{|c|c|c|}
\hline & transparent & opak \\
\hline $\begin{array}{l}\text { kanonische Buchstaben } \\
\text { für vordere Laute }\end{array}$ & $\begin{array}{l}<\text { ietpbmnIrf } \\
\text { sc vwz> }\end{array}$ & $\begin{array}{l}<d> \\
<y>\text { in }<\text { lyrik }>\end{array}$ \\
\hline $\begin{array}{l}\text { nicht-kanonische } \\
\text { Buchstaben } \\
\text { für hintere Laute }\end{array}$ & $\begin{array}{l}<\text { u a q kj }(x)> \\
<y>\text { in }<\text { yoga }>\end{array}$ & $\langle\mathrm{ho}\rangle$ \\
\hline
\end{tabular}

In (8) erscheint $<x>$ in Klammern, weil diesem Buchstaben / $\mathrm{ks} /$, eine Lautverbindung mit widersprüchlichen Artikulationsorten, entspricht, so dass $<\mathrm{x}>$ partiell transparent ist.

Die obigen Analysen des modernen lateinischen Buchstabensystems ergeben, dass Buchstabenteile und deren Merkmale nicht einzelne Laute, 
sondern ganze Lautklassen anzeigen, wobei einige lautliche Kontraste nicht repräsentiert werden. So signalisiert unserer Analyse zufolge $<j>$, dessen Kopflinie linksgerichtet, lang und nicht-gerade ist, dass dieser Buchstabe mit einem hinteren Reibelaut korrespondiert. Mehr zeigt $<j>$ nicht an. Der genaue Lautwert ergibt sich aus dem Laut- und Graphemsystem der jeweiligen Sprache, z. B. / / / im Deutschen wie in jeder oder $/ 3 /$ im Französischen wie in Jean. Aus dieser Perspektive betrachtet überrascht es nicht, dass die modernen lateinischen Buchstaben für die Verschriftung vieler Sprachen verwendet werden können.

Als Fazit der merkmalsbezogenen Analyse des modernen lateinischen Buchstabensystems halten wir fest: Drei wichtige Lautkontraste, die Schallfülle und die damit verbundene Silbenkernfähigkeit, die Artikulationsart und der Artikulationsort, korrespondieren mit Buchstabenmerkmalen. Die kanonische oder nicht-kanonische Ausrichtung von Kopf und Coda dient der Wiedergabe eines vorderen oder hinteren Artikulationsortes. Der Kontrast zwischen gerader-senkrechter und nicht gerader-senkrechter Kopflinie entspricht der Unterscheidung zwischen geschlossener und offener Artikulation. Die Länge der Kopflinie korreliert mit der Schallfülle und der damit verbundenen Silbenkernfähigkeit.

Zum Abschluss dieses Abschnitts präsentieren wir einen Vergleich mit einem künstlich geschaffenen Buchstabensystem, dem koreanischen Hangul, das bereits von Geoffrey Sampson ${ }^{23}$ als merkmalsbasiertes System eingestuft wurde. Mitte des 15. Jahrhunderts wurde unter König Sejong ein Alphabet aus 17 Konsonanten- und 11 Vokalzeichen geschaffen. Nach der traditionellen Darstellung sind die grafisch einfachsten Konsonantenzeichen vereinfachte Abbildungen der Sprechwerkzeuge (Artikulatoren) bei der Aussprache der entsprechenden Laute. Die drei ursprünglichen Vokal-Grundzeichen bilden nach kosmologischen Vorstellungen Himmel, Erde und Mensch ab. Die Buchstaben-Komposition dieses Alphabets folgt mithin nach Maßgabe des Metadiskurses über das Hangul anderen Prinzipien als die in den historisch gewachsenen Systemen nachgewiesenen. Im Hangul sind Vokalzeichen kosmologisch motiviert; Konsonantenzeichen sind Piktogramme der aktiven Artikulatoren. So deutet der Buchstabe < $<>$ für /n/ an, wie sich der Zungenkranz bei der Artikulation dieses vorderen Konsonanten zum Zahndamm hebt. Buchstaben für hintere Konsonanten, wie $<\neg>$ für $/ \mathrm{k}, \mathrm{g} /$, visualisieren, wie

23 Geoffrey Sampson: Writing Systems: A Linguistic Introduction. Stanford 1985, S. 120-140. 
sich der Zungenrücken zum Velum hebt. Der weiter oben beschriebene konstruktionelle Ikonismus im lateinischen Alphabet ist nicht piktografisch, sondern sehr viel abstrakter. Er beruht auf der systeminternen Einfachheit bzw. Kanonizität von Lauten und Buchstaben. Allerdings lässt sich auch im Hangul konstruktioneller Ikonismus nachweisen. Wir beschränken unseren Nachweis auf den konstruktionellen Ikonismus bei den Buchstaben, die mit Verschlusslauten einschließlich Nasalen korrespondieren. Vgl. (9):

(9) Konstruktioneller Ikonismus im koreanischen Hangul

\begin{tabular}{|c|c|c|c|c|}
\hline $\begin{array}{l}\text { kanonische Buchstaben } \\
\text { für vordere Laute }\end{array}$ & $\begin{array}{l}\llcorner \\
\ln /\end{array}$ & $\begin{array}{l}\ulcorner \\
/ \mathrm{t}, \mathrm{d} /\end{array}$ & $\begin{array}{l}E \\
/ t^{\mathrm{h}} /\end{array}$ & $\begin{array}{l}\text { ㄷ } \\
/ t^{\prime} /\end{array}$ \\
\hline $\begin{array}{l}\text { nicht-kanonische Buchstaben } \\
\text { für hintere Laute }\end{array}$ & $\begin{array}{l}\neg \\
/ \mathrm{k}, \mathrm{g} /\end{array}$ & $\begin{array}{l}7 \\
/ \mathrm{k}^{\mathrm{h}}\end{array}$ & $\begin{array}{l}77 \\
/ k^{\prime} /\end{array}$ & \\
\hline
\end{tabular}

Die Schreibrichtung im Hangul ist rechtsgerichtet ebenso wie die Anordnung der Silbenblöcke in Zeilen. Kanonische Buchstabenformen sind mithin ebenfalls rechtsgerichtet, $d$.h. mit nach rechts geöffnetem Winkel. Die kanonischen Buchstabenformen in (9) korrespondieren konstruktionell ikonisch mit vorderen Konsonanten, die nicht-kanonischen, linksgerichteten Buchstaben mit hinteren Konsonanten. Hier erkennen wir das Prinzip des konstruktionellen Artikulationsort-Ikonismus, das wir im lateinischen Alphabet in (8) illustriert haben, wieder. Denn die in (9) gezeigten Buchstabenmerkmale des Hangul lassen sich nicht allein aus der Position der Artikulatoren ableiten. So hätte man bspw. die Hebung des Zungenkranzes zum Zahndamm bei vorderen Lauten piktografisch transparent, allerdings konstruktionell kontra-ikonisch durch eine linksgerichtete Figur wie etwa \langle\lrcorner$\rangle$ visualisieren können. Es gibt, wie (9) zeigt, auch subtilere Ausprägungen des konstruktionellen Ikonismus im Hangul. Die lautlich einfache, nicht aspirierte und nicht glottalisierte, Artikulation wird durch einfache Formen, die komplexe aspirierte oder glottalisierte Artikulation durch komplexe Zeichen repräsentiert. Diese Buchstabenmerkmale lassen sich nicht allein aus der Position der Artikulatoren ableiten, so dass man auch im koreanischen Hangul systemgesteuerte, auf implizitem Sprachwissen beruhende Aspekte der Buchstabenorganisation vorfindet.

Um unserer Untersuchung ein höheres Maß an schriftsystemvergleichender Repräsentativität zu verleihen, ergänzen wir unsere Datengrundlage 
durch das moderne arabische und das Tifinagh-Alphabet. Die Buchstabenformen dieser Alphabete, denen wir uns in den folgenden beiden Abschnitten widmen, unterscheiden sich deutlich von den Buchstabenformen des lateinischen Alphabets und beziehen sich auf Sprachen, deren Verwandtschaftsgrad mit mitteleuropäischen Sprachen und untereinander gering ist.

\section{DAS MODERNE ARABISCHE ALPHABET}

Das arabische Alphabet ist in den ersten Jahrhunderten unserer Zeitrechnung aus den aramäischen und nabatäischen Alphabeten entstanden. ${ }^{24}$ In den arabischen Dialekten haben sich mehrere Schriftvarianten entwickelt, aber in fast allen arabischen Ländern haben sich zwei Schriftarten durchgesetzt: die so gen. Buchschrift Nas'khi und die für die schnellere Handschrift geeignete Kursivschrift Ruq'a. Für unsere Analyse haben wir die Nas'khi-Buchstabenformen in einer serifenlosen Schriftart (Arial) ausgewählt, weil diese die spezifischen strukturellen Eigenschaften der arabischen Buchstaben deutlicher hervorheben.

Viele Buchstaben des arabischen Alphabets haben bis zu vier Varianten in Abhängigkeit von ihrer Position im Wort. Die weiter unten in (11) angegebenen Buchstabenformen kommen wortinitial, aber nicht nur dort, vor. Eine linksseitige kurze untere Linie, wie etwa bei $\langle\varepsilon>$ ist lautlich nicht funktional, sondern dient als Verbindungslinie zum nächsten linksseitigen Buchstaben. Die freistehende Variante eines Buchstabens ist in mehreren Fällen symmetrisch und mithin phonologisch opaker als die wortinitiale, eindeutig linksgerichtete Form, vgl. die freistehende Variante $<$ ب > gegenüber der wortinitialen Variante $<$ ب > für $/ \mathrm{b} /$.

Im Folgenden wird eine Eigenheit des Hocharabischen angeführt, die für die Untersuchung der Korrespondenz zwischen Buchstabenform und Lautwert von Bedeutung ist. Ein charakteristisches Merkmal des Hocharabischen und anderer semitischer Sprachen ist die Tatsache, dass viele Wörter durch die Alternation der Vokale in der Wortwurzel gebildet oder flektiert werden. ${ }^{25}$ Während im Deutschen und in anderen europäischen Sprachen Konsonanten und Vokale gleichermaßen

24 Das erste datierte Zeugnis ist eine dreisprachige, griechisch-syrischarabische Inschrift aus dem Jahr 512.

25 Wolfdietrich Fischer und Otto Jastrow: Lehrgang für die arabische Schriftsprache. Wiesbaden ${ }^{5} 1996$. 
zur Konstitution der Wortwurzel beitragen, gilt dies für die Vokale im Arabischen in viel geringerem Maße. In Anbetracht dieser Tatsache überrascht es nicht, dass die Schriftsysteme der beiden Sprachen Vokale unterschiedlich behandeln. So korrespondieren 25 der 28 Buchstaben des arabischen Alphabets mit Konsonanten. Die restlichen drei Buchstaben werden für die drei Langvokale des Hocharabischen /a:/, /i:/ und /u:/ verwendet, wobei diese Buchstaben auch einen konsonantischen Wert haben können (vgl. [11] weiter unten).

Dies ist ein entscheidender Unterschied zum lateinischen Buchstabensystem. Eine erste Folgeerscheinung dieses Unterschieds ist die unterschiedliche Behandlung silbischer Strukturen in der Schrift. Wie weiter oben gezeigt, bildet die Silbe nicht nur in Silbenschriften wie der mykenischen Linear-B und der kyprischen Syllabarschrift, ${ }^{26}$ sondern auch in Buchstabenschriften eine relevante Einheit. Dabei haben Buchstabenschriften verschiedene Verfahren der Visualisierung von Silbenstrukturen entwickelt. Neben der weiter oben gezeigten regelgeleiteten Abfolge von langen und kurzen Buchstabenköpfen im lateinischen und griechischen Alphabet gibt es das Anordnen der Buchstaben in Silbenblöcken wie im koreanischen Hangul. Die Silbe spielt auch in Konsonantenschriften wie der phönizischen, hebräischen und arabischen eine zentrale Rolle. In diesen Konsonantenschriften werden Silbenränder durch Buchstaben und Silbenkerne gar nicht, wie etwa in der phönizischen Konsonantenschrift, oder durch fakultative Diakritika, wie in der modernen arabischen Konsonantenschrift, repräsentiert. Aufgrund des Verfahrens der Vokalalternation in der Flexion und Wortbildung dieser semitischen Sprachen ist die graphematische Sonderbehandlung der vokalischen Silbenkerne systemangemessen. Als Folge dieser silbenstrukturellen Besonderheit des arabischen Alphabets, das Konsonanten und Vokale grundsätzlich anders behandelt, hat sich - soweit wir es beim jetzigen Kenntnisstand beurteilen können - keine graphematische Kennzeichnung silbisch relevanter Lautkontraste bei den Konsonanten herausgebildet. Silbisch relevante Lautkontraste beziehen sich auf die Schallfülle der Laute und damit zusammenhängend auf die Unterscheidung der Konsonanten nach Artikulationsart. Während die Artikulationsart im lateinischen Alphabet,

26 Vgl. David G. Miller: Ancient Scripts and Phonological Knowledge. Amsterdam 1994; Roger D. Woodward: On the Interaction of Greek Orthography and Phonology. Consonant Clusters in the Syllabic Scripts. In: William. C. Watt (Hrsg.): Writing Systems and Cognition. Dordrecht 1994, S. 311-336. 
wie weiter oben gezeigt, durch Kopflänge und Kopfform systematisch repräsentiert wird, wird dieser Lautparameter im arabischen Alphabet nicht systematisch visualisiert. Daraus folgt auch, dass das arabische Alphabet keine Buchstaben mit einer langen, senkrechten und geraden Kopflinie herausgebildet hat. Damit bleibt die graphematische Visualisierung des Artikulationsortes der entscheidende Lautkontrast für eine Konsonantenschrift wie die arabische.

Wie im lateinischen Alphabet wird der Kontrast zwischen vorderer und hinterer Artikulation konstruktionell ikonisch kodiert: Mit wenigen Ausnahmen entsprechen vorderen Lauten kanonische Buchstabenformen, hinteren Lauten nicht-kanonische. Der Kontrast zwischen kanonischen und nicht-kanonischen Buchstabenformen ist systemspezifisch und hängt unter anderem von der Zeilenrichtung ab. Das Schriftsystem des Hocharabischen ist im Gegensatz zu den Schriftsystemen, die sich des lateinischen Alphabets bedienen, linksläufig, womit linksgerichtete und nicht wie im lateinischen Alphabet rechtsgerichtete Buchstabenformen kanonisch sind. Des Weiteren ist der Bogen, ein Halb-, Viertel- oder ganzer Kreis, im Gegensatz zu einem spitzen, geschlossenen Winkel oder geraden Strich, kanonisch. Kanonisch ist mithin eine Buchstabenform, deren Grundelement zugrunde liegend ein Bogen ist, der sich nach links öffnet oder dem sich eine linksseitige Coda anschließt. Vgl. (10):

(10) Kanonische Buchstabenstruktur im modernen arabischen Alphabet am Beispiel des Buchstabens für /b/

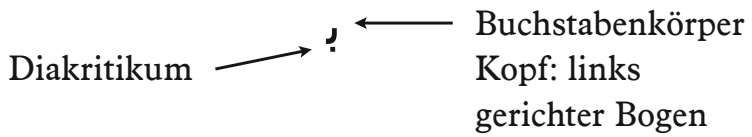

Punktförmige Diakritika, die über oder unter dem Buchstabenkörper stehen, sind seit dem 8. Jh. für 15 Konsonantenzeichen des Hocharabischen konstitutiv.

Nicht-kanonisch ist eine Buchstabenform, deren Grundelement kein Bogen (sondern ein spitzer Winkel oder ein Strich) ist oder deren Ausrichtung nicht linksgerichtet ist. Wie im lateinischen Alphabet wird der Kontrast zwischen vorderer und hinterer Artikulation konstruktionell ikonisch kodiert: Mit wenigen Ausnahmen entsprechen vorderen Lauten kanonische Buchstabenformen, hinteren Lauten nicht-kanonische. Die Tabelle in (11) illustriert den konstruktionellen ArtikulationsortIkonismus: 
(11) Konstruktioneller Artikulationsort-Ikonismus im modernen arabischen Alphabet ${ }^{27}$

Kanonische Buchstaben für vordere (präpalatale) Laute

\begin{tabular}{|c|c|c|c|c|c|c|c|c|c|c|c|}
\hline ب & ت & ث & j & נ & ز & $J$ & j & س & ش & 9 & ف \\
\hline$/ \mathrm{b} /$ & $/ \mathrm{t} /$ & $|\theta|$ & $/ \mathrm{n} /$ & $/ \mathrm{d} /$ & /ð/ & $|r|$ & $|z|$ & $/ \mathrm{s} /$ & $\mid S /$ & $\begin{array}{l}/ \mathrm{w} / \\
/ \mathrm{u}: /\end{array}$ & $/ \mathrm{f} /$ \\
\hline
\end{tabular}

\begin{tabular}{|c|c|c|c|c|c|}
\hline$J$ & 0 & $ص$ & ض & $b$ & b \\
\hline$/ I /$ & $/ \mathrm{m} /$ & $/ \mathrm{s} /$ & $/ \mathrm{d} /$ & $/ \mathrm{t} /$ & $\mid \mathrm{z} /$ \\
\hline
\end{tabular}

Nicht-kanonische Buchstaben für hintere (postalveolare) Laute

\begin{tabular}{|c|c|c|c|c|}
\hline$\varepsilon$ & $\dot{\varepsilon}$ & $>$ & $>$ & $\mathrm{I}$ \\
\hline$/ \Omega /$ & $/ \gamma /$ & $/ \hbar /$ & $/ \mathrm{x} /$ & $/ 2 / / \mathrm{a} / /$ \\
\hline
\end{tabular}

\begin{tabular}{|l|c|c|}
\hline $\begin{array}{l}\text { Opake } \\
\text { Buchstaben- } \\
\text { formen }\end{array}$ & g & $ه$ \\
\cline { 2 - 3 } & $/ \mathrm{q} /$ & $/ \mathrm{h} /$ \\
\hline
\end{tabular}

\begin{tabular}{|c|c|c|c|}
\hline \multirow{2}{*}{ Unklare Fälle } & ه. & ? & ك ك \\
\hline & $/ \mathrm{j} / / \mathrm{i}: /$ & $/ \mathrm{d} z /, / \mathrm{g} /$ & $/ \mathrm{k} /$ \\
\hline
\end{tabular}

$27 \mathrm{Zu}$ den Buchstaben-Laut-Korrespondenzen vgl. Hartmut Kästner: Phonetik und Phonologie des modernen Hocharabisch. Leipzig 1981; Wolfdietrich Fischer und Otto Jastrow: Lehrgang für die arabische Schriftsprache. Wiesbaden ${ }^{5}$ 1996. Die phonetischen Transkriptionen der Laute richten sich nach dem aktuell gültigen Internationalen Phonetischen Alphabet, lediglich bei der Notation der emphatischen (velarisierten) Konsonanten folgen wir der Notationskonvention der Semitistik und notieren sie mit einem Punkt unterhalb des Lautzeichens (Wolfdietrich Fischer und Otto Jastrow: Lehrgang für die arabische Schriftsprache. Wiesbaden ${ }^{51996) . ~}$ 
Eindeutig opak sind nur zwei Buchstaben. Dies sind die kanonischen Buchstabenformen $\langle\ddot{g}\rangle$ und $\langle\boldsymbol{Q}\rangle$ für die hinteren Laute /q/ bzw. /h/. Es gibt auch drei unklare Fälle. Die kanonische Form $<>$ ist für den palatalen Halbvokal /j/ opak, für den vorderen Vokal /i:/ transparent. Die nicht-kanonische Form < > > ist für die alveolare Affrikate /dz/ opak, nicht jedoch für den hinteren Laut /g/, der in lokalen Varianten des Arabischen und im Klassischen Arabisch anstelle von /3/ verwendet wird. Schließlich ist die Ausrichtung der wortinitialen Buchstabenvariante $\langle\zeta\rangle$ für den hinteren Laut /k/ unklar; allerdings ist die freistehende, kanonisch ausgerichtete Buchstabenvariante < ك > für die hintere Artikulation opak. In der Tabelle für unklare Fälle wurde die Quelle der Opazität grau hinterlegt. Wir verzichteten darauf, $<9>$ für den labio-velaren Halbvokal /w/ und den hinteren Vokal /u:/ als unklar zu klassifizieren. Dies liegt daran, dass beide Laute mit einer Lippenrundung ausgesprochen werden und daher vordere Artikulatoren involvieren. Die Beteiligung der Lippen scheint für diese Buchstabenform maßgeblich zu sein, wie die Korrespondenz für denselben Buchstabenkörper, nämlich $\langle\dot{9}>$, für den labio-dentalen Laut /f/ belegt.

In (12) und (13) illustrieren wir den konstruktionellen Artikulationsort-Ikonismus anhand von Wortbeispielen in der phonetisch transparenteren Arialschriftart und in der phonetisch opakeren Timesschriftart. Die Opazität der letzteren liegt daran, dass in ihr der Unterschied zwischen Bogen und Winkel weniger deutlich zum Vorschein kommt. Die Transliteration der Buchstabenfolge im phonetischen Alphabet mit der Angabe der linksgerichteten Schreib- und Leserichtung dient als zusätzliche Hilfestellung.

(12) Drei kanonische Buchstaben für drei vordere Konsonanten

\begin{tabular}{|c|c|c|}
\hline 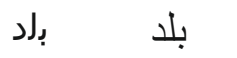 & $/ \mathrm{dlb} /$ & /balad/ ,Land \\
\hline درس & $/ \mathrm{srd} /$ & $\begin{array}{l}\text { /darasa/ ,studieren“ oder } \\
\text { /darrasa/ ,lehren“ }\end{array}$ \\
\hline
\end{tabular}

(13) Zwei kanonische Buchstaben für zwei vordere Laute und ein nichtkanonischer Buchstabe für einen hinteren Laut (grau hinterlegt)

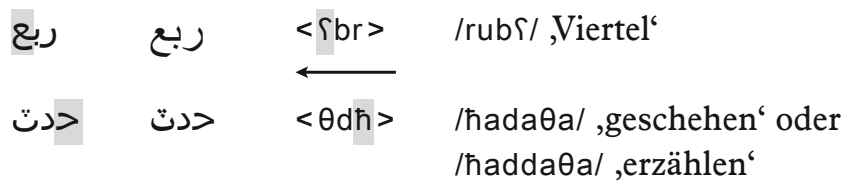


Die Beispiele enthalten auch wortmediale und wortfinale Buchstabenformen, die sich von den in (11) illustrierten wortinitialen Formen unterscheiden. Dennoch zeigen die Beispiele sehr deutlich, dass die Folge kanonischer Buchstaben in (12) ausschließlich aus linksgerichteten Bögen besteht, während in (13) das erste Wort mit einem rechtsgerichteten Bogen endet und das zweite Wort mit einem spitzen Winkel beginnt.

Zusammenfassend lässt sich für das arabische Alphabet feststellen, dass der Kontrast zwischen Bogen und Nicht-Bogen sowie die Öffnungsrichtung eines Bogens bei 23 von 28 Buchstaben den vorderen oder hinteren Artikulationsort konstruktionell ikonisch indizieren. Von den fünf restlichen Buchstaben sind nur zwei eindeutig opak.

\section{DAS TIFINAGH-ALPHABET DER HOGGAR-TUAREG}

Bereits mehr als 500 Jahre vor der Zeitenwende haben Berbervölker für die regionalen Dialekte ihrer Sprache, die nicht mit dem Arabischen verwandt ist, eigene Konsonanten-Alphabete entwickelt. Diese Alphabete werden in der Forschung als Libyco-Berber-Alphabete klassifiziert. Die Buchstaben dieser Alphabete sind auf zahllosen Inschriften in vielen Gebieten Nordafrikas und auf den Kanarischen Inseln erhalten. Ob die Berber die phönizischen Schriftzeichen nur modifiziert haben, lediglich die Idee einer Konsonantenschrift von den Phöniziern übernommen haben oder ob das Libyco-Berber eine autochthone Entwicklung ist, ist in der Wissenschaft umstritten. ${ }^{28}$ Diese Schrift ist ab dem 6. Jh. n. Chr. bei den Berbervölkern der Küstenregionen und im Norden Afrikas außer Gebrauch gekommen. Die Zeichen haben jedoch die Jahrhunderte der kulturellen Überfremdung in Nordafrika durch Römer, Araber und Franzosen bei den nomadisierenden Tuareg überlebt, die ihr Alphabet Tifinagh

28 Zur Diskussion über Alter und Herkunft der Tifinagh-Alphabete vgl. Salem Chaker und Slimane Hachi: À propos de l'origine et de l'âge de l'écriture libyco-berbère. In: Salem Chaker (Hrsg.): Études berbères et chamito-sémitiques, Mélanges offerts à Karl-G. Prasse. Paris, Louvain 2000, S. 95-111; Lionel Galand: Un vieux débat: l'origine de l'écriture libyco-berbère, In: Lettre de l'association des amis de l'art rupestre Saharien 20 (2001), S. 21-24; Salem Chaker: L'écriture libyco-berbère. État des lieux, déchiffrement et perspectives linguistiques et sociolinguistiques. Lyon 2002. Dort finden sich auch zahlreiche weitere Literaturhinweise. 
nennen..$^{29}$ Allerdings hat sich das Tifinagh in dieser Zeit auch diversifiziert und die Alphabete der einzelnen Tuareg-Völker werden jeweils durch Zeichen ergänzt, welche den spezifischen Lauten der Dialekte entsprechen. Andererseits repräsentieren dieselben Zeichen bei unterschiedlichen Volksstämmen teilweise andere Laute. Eine Ursache für diese Diversität ist darin zu sehen, dass das Tifinagh in der oralen Kultur der Nomaden nicht für die Kommunikation zwischen den Stämmen benutzt wurde, sondern als internes Wissen der Stämme für kurze Botschaften oder geheime Notizen verwendet und von Generation zu Generation weitergetragen wurde. ${ }^{30} \mathrm{Im}$ Rahmen des Wiedererwachens der kulturellen Identität der Tuareg und anderer Berbervölker in den 7oer Jahren des 20. Jh. ist das Tifinagh verstärkt wissenschaftlich dokumentiert und analysiert worden und dabei wurden auch die Libyco-Berber-Alphabete als gemeinsame Vorläufer des Tifinagh mit ihren Lautwerten rekonstruiert. Nach langen Diskussionen über Alternativen zu einer Einheitsschrift für die Berbervölker wurde aus mehreren Alphabeten der Tuareg das standardisierte Neo-Tifinagh kompiliert, das nunmehr mit 33 Buchstaben auch Zeichen für Vokale und arabische bzw. europäische Fremdlaute enthält. ${ }^{31}$

Da das Neo-Tifinagh nicht als historisch tradiertes, sondern vielmehr als künstlich geschaffenes Buchstabensystem betrachtet werden muss, ist dieses Alphabet für unseren Untersuchungszweck wenig geeignet. Für die Untersuchung einer phonologischen Ikonizität des Tifinagh ziehen wir daher das tradierte Alphabet derjenigen Tuareg-Stämme heran, die im Hoggar-Gebirge im Süden Algeriens im Grenzgebiet zu den Staaten Niger und Mali nomadisieren. Die Hoggar-Tuareg unterlagen am wenigsten von allen Berbervölkern dem Einfluss fremder Kulturen. Ihr Alphabet kann daher als ein von arabischen Einflüssen möglichst unverfälschtes traditionelles Buchstabensystem eingestuft werden.

Die 24 Buchstaben des Hoggar-Tifinagh repräsentieren 22 Konsonanten und zwei Halbvokale. Sie stehen ohne Bindung nebeneinander. Aus dem Konsonantencharakter des Alphabets leiten wir (wie im Fall

29 Die Tuareg selbst glauben, dass sie ihr Alphabet einem mythischen Stifter verdanken, vgl. Hélène Cloudot-Hawad: Les Tifinagh comme écriture du détournement: usages touaregs du XXI siècle. In: Études et Documents Berbères 23 (2005), S. 5-30, hier S. 5.

30 Cloudot-Hawad: Les Tifinagh comme écriture du détournement (wie Anm. 29), S. 7.

31 Vgl. dazu das Autorenkollektiv IRCAM, Institut Royal de la Culture Amazighe: Initiation à la langue Amazighe. Rabat 2004, S. 14. 
des Arabischen) ab, dass silbenstrukturell relevante Lautkontraste nicht aus der Form der Konsonantenbuchstaben, sondern aus der Präsenz versus Absenz eines Buchstabens für einen Konsonanten bzw. einen Vokal ableitbar sind. Damit bleibt die graphematische Visualisierung des Artikulationsortes der entscheidende Lautkontrast für die Konsonantenbuchstaben des Tifinagh.

Wie im lateinischen und arabischen Alphabet wird auch im untersuchten Tifinagh-Alphabet der Kontrast zwischen vorderer und hinterer Artikulation konstruktionell ikonisch kodiert: Abgesehen von wenigen Fällen entsprechen vorderen Lauten kanonische Buchstabenformen, hinteren Lauten nicht-kanonische. Dabei ist die Unterscheidung zwischen kanonischen und nicht-kanonischen Buchstabenformen systemspezifisch. Im Tifinagh gibt es keine vorgegebene Zeilenrichtung. ${ }^{32}$ Die historisch lang anhaltende Absenz einer standardisierten Zeilenrichtung korreliert mit der Tatsache, dass die Buchstaben des Tifinagh meist symmetrisch sind und nur in Ausnahmefällen eine Orientierung haben. ${ }^{33}$ Daher kann die Kanonizität der Buchstaben nicht, wie im lateinischen und arabischen Alphabet, aus der Zeilenrichtung abgeleitet werden. Vielmehr ist die Geschlossenheit des Buchstabens bzw. deren Absenz das entscheidende Kriterium. Wir erinnern uns daran, dass auch im lateinischen Alphabet kanonische Buchstaben möglichst geschlossene strichhaltige Figuren bilden. Dies unterscheidet nicht nur lateinische Buchstaben von Interpunktionszeichen wie $\langle$ : $>$ und $<;>$, sondern auch geschlossene Buchstaben für vordere Laute wie $<\mathrm{b}>$ von offenen Buchstaben für hintere Laute wie $<\mathrm{k}>$. Dieser Buchstabe ist insoweit offen, als sich Kopf und Coda voneinander abwenden. Das Kriterium der Geschlossenheit wenden wir für das Tifinagh so an, dass wir strichlose Buchstaben, die nur aus unverbundenen Punkten bestehen, als nicht-kanonisch, die strichhaltigen Buchstaben als kanonisch klassifizieren. In (14) zeigen wir, dass abgesehen von wenigen Fällen vorderen Lauten kanonische Buchstabenformen, hinteren Lauten nicht-kanonische Buchstabenformen entsprechen.

32 Als Lesehilfe für die Zeilenrichtung dienen zwischen den überwiegend symmetrischen Zeichen einzelne gerichtete Buchstaben. Für bestimmte Lautkombinationen, insbes. solche mit $/ \mathrm{n} /$, werden Ligaturen verwendet. Sie können als zusätzliche Indikatoren für die Zeilenrichtung dienen.

33 Zur Korrelation zwischen variabler Zeilenrichtung und Buchstabensymmetrie in der Geschichte des griechischen und lateinischen Alphabets vgl. Primus: Die Buchstaben unseres Alphabets (wie Anm. 20). 
(14) Konstruktioneller Artikulationsort-Ikonismus im Tifinagh-Alphabet der Hoggar-Tuareg

Kanonische Buchstaben für vordere (präpalatale) Laute

\begin{tabular}{|c|c|c|c|c|c|c|c|c|c|c|}
\hline$\circ$ & $\ominus$ & $\odot$ & $\odot$ & $w$ & $x$ & $I$ & + & ][ & $\#$ & $H$ \\
\hline$/ \mathrm{r} /$ & $/ \mathrm{b} /$ & $/ \mathrm{s} /$ & $/ \mathrm{J} /$ & $/ \mathrm{z} /$ & $/ 3 /$ & $/ / /$ & $/ \mathrm{t} /$ & $/ \mathrm{f} /$ & $/ \mathrm{z} /$ & $/ \mathrm{n} /$ \\
\hline
\end{tabular}

\begin{tabular}{|c|c|c|c|}
\hline$\sqsubset$ & $E$ & $E$ & $\Lambda$ \\
\hline$/ \mathrm{m} /$ & $/ \mathrm{d} /$ & $/ \mathrm{t} /$ & $/ \mathrm{d} /$ \\
\hline
\end{tabular}

Nicht-kanonische Buchstaben für hintere (postalveolare) Laute

\begin{tabular}{|c|c|c|c|c|}
\hline$\cdots$ & $\vdots$ & $\because$ & $::$ & $\vdots$ \\
\hline$/ \mathrm{q} /$ & $/ \mathrm{\gamma} /$ & $/ \mathrm{k} /$ & $/ \mathrm{X} /$ & $/ \mathrm{h} /$ \\
\hline
\end{tabular}

\begin{tabular}{|l|c|c|}
\hline $\begin{array}{l}\text { Opake Buchstaben } \\
\text { (kanonische Formen } \\
\text { für hintere Laute) }\end{array}$ & $\leqslant$ & $\mathrm{X} / \mathrm{j} /$ \\
\cline { 2 - 3 }$/ \mathrm{g} /{ }^{34}$ \\
\hline
\end{tabular}

\begin{tabular}{|l|c|c|}
\hline Unklare Fälle & $:$ & $\uparrow$ \\
\cline { 2 - 3 } & $/ \mathrm{w} /$ & $/ \mathrm{d} 3 /$ \\
\hline
\end{tabular}

Die unklaren Fälle bedürfen eines Kommentars. Der strichlose Buchstabe für den labio-velaren Halbvokal /w/ ist nicht-kanonisch. Damit wäre die velare Komponente des Lautes ikonisch abgebildet, die labiale nicht ikonisch. Der Buchstabe für die präpalatale Affrikate $/ \mathrm{d} z /$ enthält einen Strich, womit man ihn als kanonischen Buchstaben, der konstruktionell ikonisch diese vordere Affrikate abbildet, einstufen könnte. Allerdings enthält der Buchstabe zwei unverbundene Punkte, die bei Fehlen eines Striches nicht-kanonische Buchstaben charakterisieren. Daher muss das Kriterium der Punkthaltigkeit beim jetzigen Wissensstand vage bleiben.

34 In den Quellen zum Tifinagh wird dieser Laut als frikativiert, auch als „g doux“, bezeichnet und als /g'/ oder/g/transkribiert. 
Es bleibt mithin offen, ob es als Absenz von Strichen oder als Präsenz von Punkten formuliert werden muss. In (14) haben wir die erste Variante dieses Kriteriums angewandt, um die Ergebnisse nicht ggf. zu unseren Gunsten zu verfälschen.

Zusammenfassend lässt sich für das Tifinagh-Alphabet der HoggarTuareg feststellen, dass der Kontrast zwischen strichhaltigen und strichlosen Buchstaben bei 20 von 24 Buchstaben den vorderen oder hinteren Artikulationsort konstruktionell ikonisch indiziert. Zwei Buchstabenformen sind opak, zwei schwer einzuordnen. Auch muss beim jetzigen Wissensstand offen bleiben, ob sich ein nicht-kanonischer Buchstabe durch die Absenz von Strichen oder durch die Präsenz von Punkten auszeichnet.

\section{ZUSAMMENFASSUNG}

Buchstaben sind Symbole und nicht Piktogramme. Wie bei jedem Symbol stehen Form und Funktion grundsätzlich in einem arbiträren, nicht unmittelbar transparenten Verhältnis zueinander. Allerdings entwickeln Symbolsysteme, so auch historisch tradierte Buchstabensysteme, funktionale Transparenz durch Kompositionalität und konstruktionellen Ikonismus. Die phonologische Funktion eines Buchstabens lässt sich zu einem hohen Maß aus seinen Bestandteilen, seinen Formmerkmalen sowie aus der Art und Weise, wie diese Bestandteile zusammengefügt sind, ableiten.

In allen untersuchten historisch tradierten Alphabeten, im lateinischen und arabischen Alphabet ebenso wie im Tifinagh, konnten wir Kompositionalität nachweisen. Bestimmte phonologische Funktionen lassen sich aus den Merkmalen bzw. Komponenten der Buchstaben und der Art ihrer Verknüpfung bestimmen. Für das lateinische und arabische System konnten wir die mehrheitlich asymmetrischen Buchstaben in Kopf und Coda zerlegen. Für das Tifinagh müssen zukünftige Untersuchungen klären, ob eine solche asymmetrische Zerlegung der mehrheitlich symmetrischen Buchstaben fruchtbringend ist. Das lateinische Alphabet hebt sich von den anderen beiden untersuchten Konsonanten-Alphabeten dadurch ab, dass es gleichermaßen Konsonanten und Vokale durch Buchstaben repräsentiert und silbenstrukturell relevante Kontraste, zu denen neben der Konsonant-Vokal-Unterscheidung auch die Artikulationsart gehört, durch die Komposition der Buchstabenteile und deren Merkmale abbildet.

Alphabetübergreifend lässt sich eine konstruktionell ikonische Repräsentation des Kontrastes zwischen vorderer und hinterer Artikulation 
nachweisen. Mit wenigen Ausnahmen ${ }^{35}$ entsprechen vorderen Lauten kanonische Buchstabenformen, hinteren Lauten nicht-kanonische. Dabei ist die Unterscheidung zwischen kanonischen und nicht-kanonischen Buchstabenformen systemspezifisch. Nichtsdestotrotz haben wir zwei Kriterien entdeckt, die in mehreren Alphabeten gemeinsam oder getrennt gelten. Erstens sind kanonische Buchstaben gerichtet, und zwar in der Schreib- bzw. Leserichtung der Zeile. Zweitens bilden kanonische Buchstaben möglichst geschlossene Figuren. Für das lateinische Alphabet gelten beide Kanonizitätsmerkmale. Für das arabische Alphabet gilt die (aufgrund der Zeilenrichtung linksseitige) Buchstabenrichtung, für das Tifinagh gilt die Geschlossenheit der Figur als Merkmal kanonischer Buchstaben. Hinzu kommen systemspezifische Kriterien. Im arabischen Alphabet ist neben der linksseitigen Buchstabenausrichtung auch der Bogen ein Merkmal kanonischer Buchstaben. Im Tifinagh kommt die besondere Auslegung des Geschlossenheitskriteriums als systemspezifische Eigenschaft hinzu: Es gibt im Gegensatz zum lateinischen Alphabet strichlose Buchstaben, so dass die Präsenz oder Absenz von Strichen (bzw. Punkten) dieses Kriterium ausmacht.

Konstruktioneller Ikonismus ist nicht piktografisch, sondern viel abstrakter. Er beruht nicht auf einer piktografischen Abbildung der Artikulatoren bei der Produktion und Wahrnehmung der entsprechenden Laute, die im koreanischen Hangul, einer erst im 15. Jh. künstlich erschaffenen Schrift, die Buchstabenformen maßgeblich bestimmt. Vielmehr beruht konstruktioneller Ikonismus auf der systeminternen Einfachheit bzw. Kanonizität von Lauten und Buchstaben. Dabei spielen, wie in einem selbstorganisierenden System erwartet, die Relationen der Buchstaben zueinander als inner-graphematische Kontraste und die Relationen der Laute zueinander als systeminterne phonologische Kontraste eine entscheidende Rolle. Erst auf dieser Grundlage treten die beiden Systeme, Buchstabensystem und Lautsystem, in eine regelhafte Beziehung zueinander, die wir hier als konstruktionellen Ikonismus ausgemacht haben.

35 Verletzungen des Kompositionalitätsprinzips kommen auch in Lautsprachen vor. So haben wir viele kompositionell transparente Zusammensetzungen wie Türgriff, dessen Bedeutung aus der Bedeutung seiner Bestandteile Tür und Griff und der Art ihrer Verknüpfung mit Griff als Kopf ableitbar ist. Es gibt aber auch semantisch opake Zusammensetzungen wie Fernseher, dessen Bedeutung nicht aus der Bedeutung von fern und Seher ermittelt werden kann. 
STEFAN CLAUDIUS

\section{SCHRIFTPRODUKTION HEUTE}

\section{TECHNISCHE GEGEBENHEITEN}

Seit der Entwicklung der beweglichen Bleilettern verändern sich die Methoden zur Herstellung von Schriften ${ }^{1}$ kontinuierlich. Stets waren sie eng mit den sich ebenfalls verändernden Satztechniken verbunden, und die technischen Möglichkeiten Schrifttypen herzustellen, beeinflussten dabei die Gestalt der Buchstaben. Das historische Spektrum reicht hierbei vom manuellen Buchstabenschnitt bis zum digital codierten Beschreibungsverfahren. Das konkrete Aussehen der Buchstaben hängt also zumindest in den Gestaltungsdetails von den technischen Möglichkeiten derjenigen $\mathrm{ab}$, die die Buchstaben tatsächlich herstellten. Was also heißt es, einen Buchstaben zu formen, ihn anzufertigen?

Die ersten europäischen Drucktypen wurden ab der Mitte des 15. Jahrhunderts aus Blei hergestellt. Die einzelnen Bleilettern wurden so lange wiederverwendet, bis sie sich abgenutzt hatten - besonderen Wert hatten also die Gussformen. Zur Erzeugung einer Bleiletter goss man Blei in eine Form, an deren unterem Ende eine Matrize eingespannt war, deren Vertiefung das Blei aufnahm und im Abguss die druckende Erhöhung des Buchstabens ergab. Zur Herstellung der Schriftmatrize schlug man in eine Kupfer- oder Messingplatte die Form eines Buchstabens ein. Zum Einschlagen brauchte man zunächst eine spiegelverkehrte Letter aus Stahl, die sogenannte Patrize. Der Stempelschneider gestaltete durch die Bearbeitung eines Stückes Metall Buchstabe für Buchstabe und Schriftgröße für Schriftgröße. Nach heutigem Verständnis würde man sagen, dass jeder Schriftgrad eine eigenständige Schrift darstellte, weil

$1 \mathrm{Im}$ engeren Sinn ist die Herstellung von Schriftarten gemeint, also die Produktion von für den Druck bestimmten Drucktypen. 
jede leicht unterschiedliche Proportionen und jedes Mal einen anderen Strichstärkenkontrast aufwies. ${ }^{2}$ Diese Unterschiedlichkeit in der Ausführung der Schriften war der Erkenntnis geschuldet, dass zu dünne Striche in sehr kleinen Schriftgraden optisch ,aufreißen', man sie also schlechter wahrnimmt. In größeren Graden ließ sich eine sehr viel höhere Präzision erreichen, und man nutzte diese dazu, die Haarlinien ${ }^{3}$ in Relation zu den Schattenlinien dünner zu schneiden. ${ }^{4}$

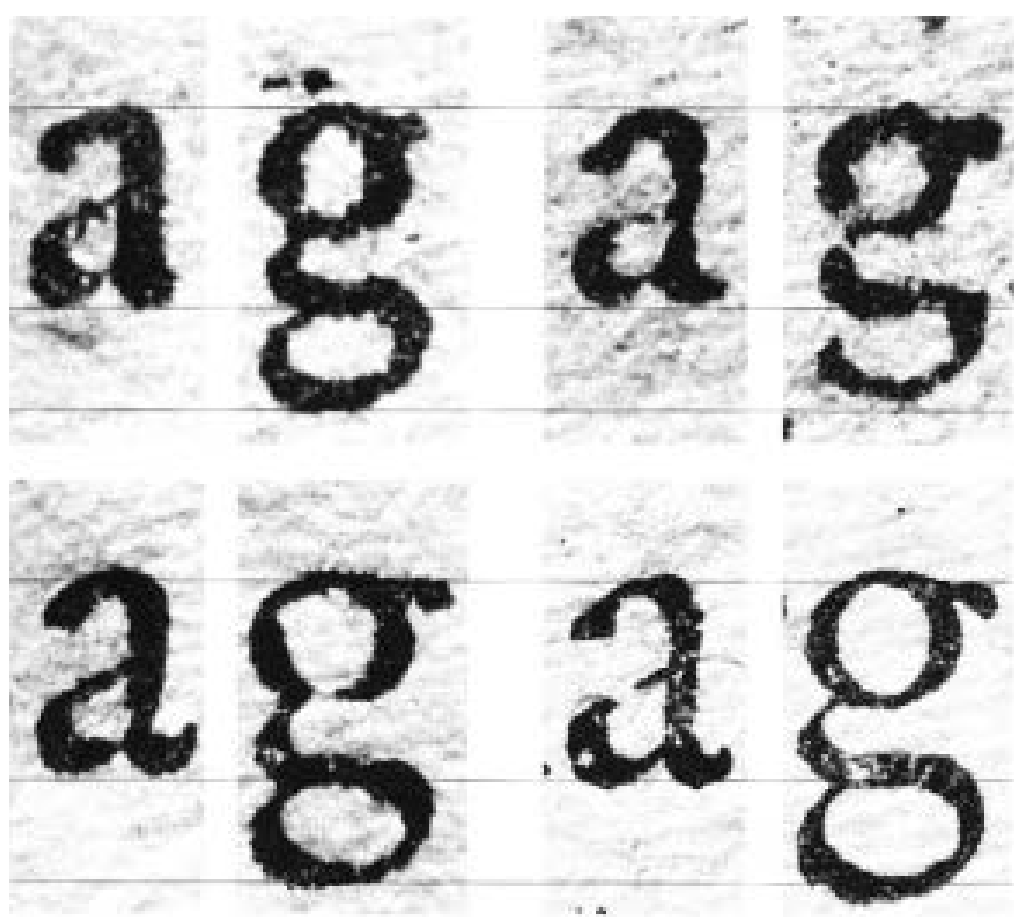

1 Vier unterschiedliche Bleisatzschriftgrade auf die gleiche Größe skaliert. Aufsteigender Schriftgrad von links oben nach rechts unten: Nonpareille Romaine, Coronelle Romaine, Philosophie Romaine, Augustine Romaine. Man sieht deutlich, wie der Strichstärkenkontrast zunimmt und die Zeichnung immer präziser wird.

2 Vgl. Fred Smeijers: Counterpunch. London 1996, S. 133-139.

3 Die frühen Drucktypen leiteten sich in ihrer Gestaltung von der Kalligrafie ab. Durch die Schrägstellung der Schreibfeder ergaben sich je nach Richtung eines Striches dickere oder dünnere Linien. Die dünnen heißen Haarlinien, die dicken Schattenlinien.

4 Vgl. Philip B. Meggs, Roy McKelvey (Hrsg.): Revival of the Fittest. New York 2000, S. 19. 
Die frühen Stempelschneider waren oft ursprünglich Goldschmiede oder Graveure, die sich irgendwann in ihrer Arbeit auf die Herstellung von Buchstabenstempeln spezialisierten ${ }^{5}$. In der Tat erforderte die Arbeit vor allem eine hohe handwerkliche Spezialisierung und viel praktisches Wissen, das vor allem persönlich weitergegeben wurde. ${ }^{6}$ Die Formung der Buchstaben wurde auch eher als praktisches denn als gestalterisches Problem wahrgenommen.

Obwohl sich im Laufe der Zeit das Verfahren der Matrizenherstellung veränderte, blieb doch das Grundprinzip des Stempelschneidens bis zum 19. Jahrhundert ${ }^{7}$ im Wesentlichen das Gleiche. Zu dieser Zeit begann sich die Gestaltung der Buchstaben von ihrer Produktion abzukoppeln, so dass Gestalter die Buchstaben zunächst vorzeichneten und diese dann von Spezialisten in eine Schrift umgesetzt wurden. ${ }^{8}$

Mit der Einführung des Fotosatzes ab Mitte der 1960er Jahre wurde es möglich, Buchstaben direkt auf einen Film zu belichten. Dazu bedurfte es einer Schablone mit einem Satz aller Buchstaben der jeweiligen Schrift. Zur Belichtung rotierte diese an die entsprechende Buchstaben-Position. Mit diesem Verfahren konnte man nun nun auch Skalierungen und Verzerrungen der Buchstaben vornehmen. Während man für den Bleisatz jede Schriftgröße einzeln anfertigen musste, war es nun möglich mit einer Schriftschablone (vgl. Abb. 2) viele unterschiedliche Schriftgrößen darzustellen. Außerdem wurde die Herstellung einer Schrift wesentlich vereinfacht. Man brauchte lediglich eine sauber gezeichnete Vorlage jedes einzelnen Buchstabens. Diese wurde abfotografiert und auf die Größe der Schablone verkleinert. ${ }^{9}$ Dieses Verfahren zur Schriftherstellung war damit sehr viel preiswerter und einfacher als es die Herstellung von Bleilettern oder Matrizen gewesen war. Dadurch kamen viele neue Schriften mit eher illustrativem Charakter auf den Markt, die nicht für den Mengensatz geeignet waren. Man darf bezweifeln, dass solche Schriften in dieser Menge erschienen wären, wenn sie aufwändig und teuer für den Bleisatz hätten hergestellt werden müssen. ${ }^{10}$

5 Vgl. Harry Carter: A View of Early Typography up to about 160o. London 2002, S. 102.

6 Eine der wenigen Anleitungen stellt das Manuel Typographique von Pierre Simon Fournier von 1764 dar.

7 Vgl. Smeijers: Counterpunch (wie Anm. 2), S. 118.

8 Vgl. ebd.

9 Vgl. Meggs, McKelvey (Hrsg.): Revival of the Fittest (wie Anm. 4), S. 26.

10 Als Beispiel mögen vielleicht die Schriften von Roger Excoffon dienen, die zum größten Teil als Fotosatz-Schriften erschienen und zu ihrer Zeit 


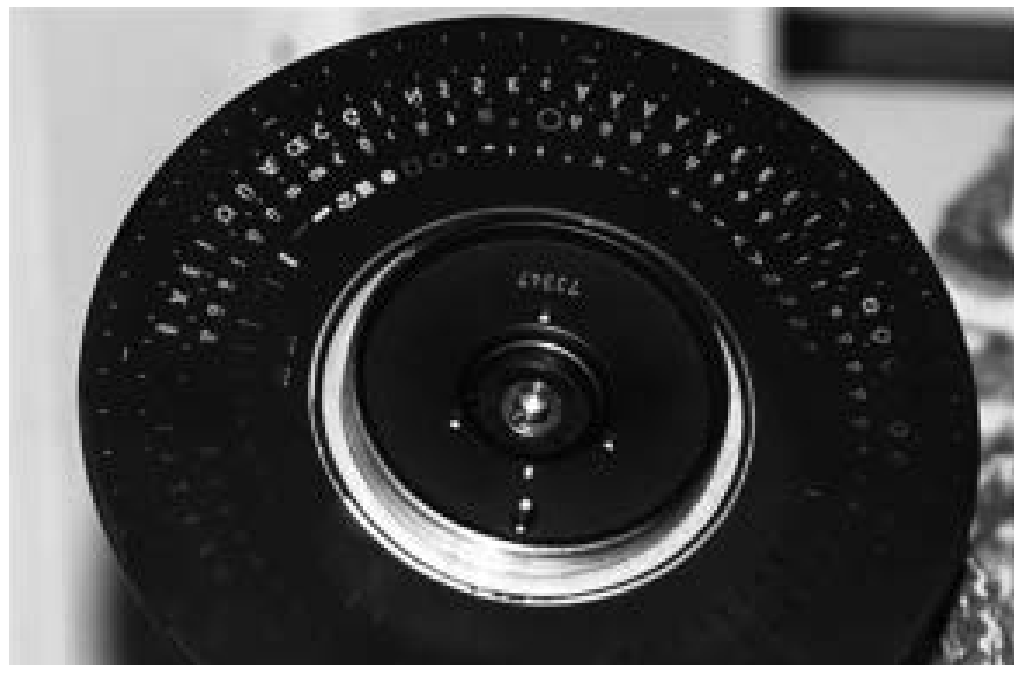

2 Schriftscheibe der Diatype der Berthold AG, Markeinführung 1960

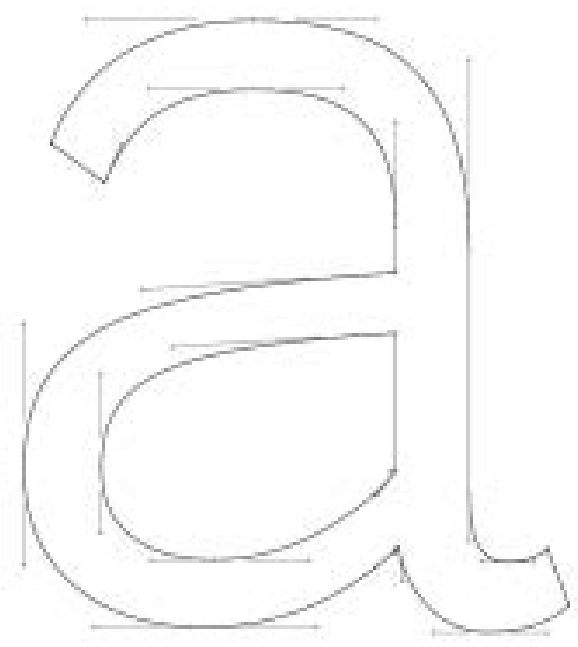

3 Darstellung eines Buchstabens mit seinen Ankerpunkten und Vektoren in der Kurvenbeschreibungssprache PostScript

recht beliebt waren, vgl. Sebastian Carter: Twentieth Century Type Designers. Veenendall 1987 , S. $131 \mathrm{f}$ und S. $135 \mathrm{f}$. 
Ab Mitte der 1980er Jahre setzte sich das Desktop-Publishing durch. Texte wurden am Computer gesetzt und die zu belichtenden Schriften mit einem Laser auf den Druckfilm belichtet. Dazu brauchte man eine digitale Beschreibung des Buchstabens, die entweder über Punkte, Striche oder Vektoren stattfinden konnte.

Von den Möglichkeiten der digitalen Beschreibung ist die vektorielle (Abb. 3) die präziseste und hat sich mittlerweile durchgesetzt. ${ }^{11}$ Um eine Schrift zu erstellen in der für jeden Buchstaben Vektoren definiert werden, bedarf es eines speziellen Programms, das einerseits die Werkzeuge zur Verfügung stellt um die jeweiligen Buchstaben zu zeichnen, andererseits aber auch die Möglichkeit bietet, die Abhängigkeiten der Buchstaben untereinander zu definieren. Die erste Abhängigkeit sind die Abstände der Lettern zueinander. Vor und hinter jedem Buchstaben gibt es Leerräume, das so genannte Fleisch. In dem Zurichtung genannten Prozess werden die Weißräume definiert. ${ }^{12}$ Ziel ist dabei, die Räume so festzulegen, dass auch Buchstaben ganz unterschiedlicher Ausformungen in optisch ähnlichen Abständen zueinander stehen. In einem Wort ergeben sich zwischen den Buchstaben viele unterschiedlich ausgeformte Weißräume. Ziel des Schriftgestalters ist es, diese in ein harmonisches Gleichgewicht zu bringen, so dass es nicht zwischen einigen Buchstaben einen sehr kleinen und zwischen zwei anderen einen sehr großen Weißraum gibt. ${ }^{13}$ Trotz aller Bemühungen kann es jedoch Buchstabenkombinationen geben, deren Formen sich so ungünstig zueinander verhalten, dass ein übermäßiger Weißraum entsteht. Um auch diese Spezialfälle ästhetisch zufriedenstellend setzen zu können, werden Unterschneidungen (vgl. Abb. 4) definiert, die jeweils nur ein Buchstabenpaar betreffen. Darüber hinaus gibt es seit der Einführung von OpenType ${ }^{14}$ die Möglichkeit unterschiedliche

11 Bei einer vektoriellen Beschreibung wird ein Pfad mittels Ankerpunkten und Kurven definiert. Eine Alternative stellt z. B. die Darstellung eines Buchstabens in einzelnen Punkten dar, man spricht von BitmapSchriften.

12 Zunächst durch die Festlegung einer Vorbreite, also dem Fleisch vor einem Buchstaben und der Nachbreite, dem Fleisch hinter dem Buchstaben. Im zweiten Schritt erst folgt die Festlegung von Unterschneidungen, im Englischen Kerning.

13 Vgl. Jost Hochuli: Das Detail in der Typografie. Sulgen 2005, S. 30 f.

14 OpenType wurde 1996 eingeführt, seit 2000 wuchs die Bedeutung und Verwendung, heute sind OpenType Schriften defacto Standard. Sie funktionieren Plattformübergreifend und erlauben u. a. durch die Unicode Adressierung sehr viel mehr Zeichen in einer Schrift unterzubringen als 
Zusatzfunktionen in einen Font ${ }^{15}$ hinein zu programmieren. ${ }^{16}$ Typische Anwendungsbereiche sind die Einbindung alternativer Buchstabenformen

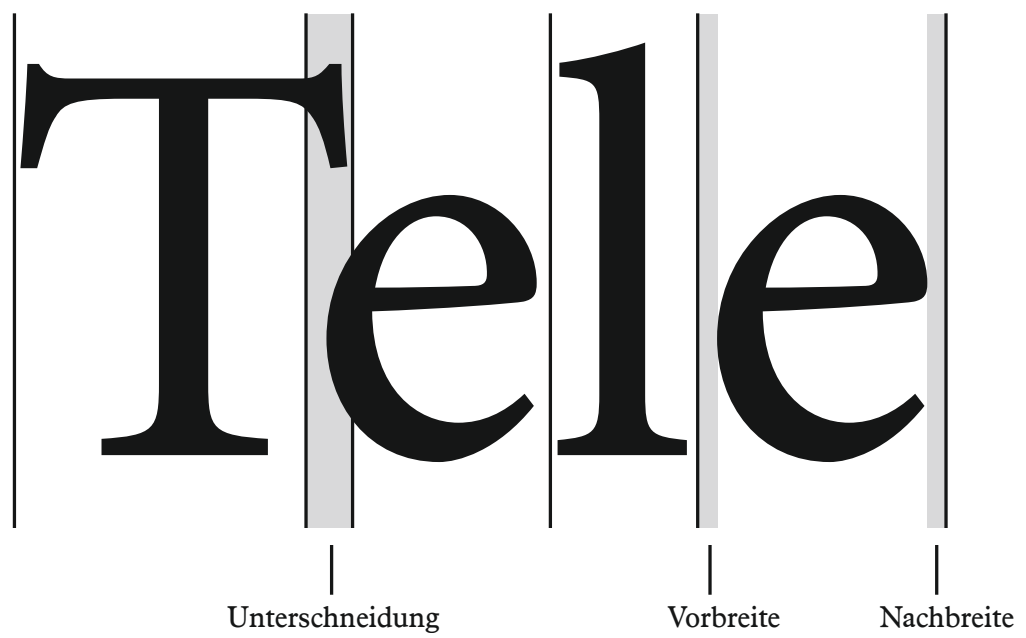

4 Jeder Buchstabe hat eine so genannte Vor- und Nachbreite auch Fleisch genannt. Um unerwünschte Weißräume zu verhindern, werden Unterschneidungen wie zwischen $\mathrm{T}$ und e definiert.

bei vorherigen Formaten, was sie auch für umfangreichere nichtlateinische Schriftsysteme geeignet macht. Seit der jüngesten Programmversion unterstützen auch Officeprogramme von Microsoft (dem ursprünglichen Entwickler des Formates) eine begrenzte Anzahl von OpenType-Features. Vgl. http://de.wikipedia.org/wiki/OpenType (24.1.2013).

15 Mit einem Font ist eine einzelne Schriftdatei gemeint. Der Begriff Schrift oder Schriftart beschreibt eine Gestaltungseinheit. Bei einer Schriftfamilie kann diese Gestaltungseinheit aus mehreren Schnitten bestehen, die Variationen dieser Gestaltung darstellen. Jeder dieser Schnitte wird als einzelner Font generiert und zur Benutzung installiert. Der Begriff Font leitet sich vom Englischen Fount ,Schmelzung' ab und bezeichnete im Bleisatz eine Schriftgröße einer Schriftart, also z. B. Bembo Italic $9 p t$, was dem Inhalt eines Setzkastens entsprach. Im Deutschen müsste man korrekterweise immer von Glyphensätzen sprechen, doch ist dieser Ausdruck eher ungewöhnlich. 16 So genannte OpenType-Features erlauben über einfache Ersetzungsfunktionen, Sets von Buchstaben gegeneinander auszutauschen. Dadurch kann man, sofern die Zeichen in der Schrift angelegt sind, z. B. Text in echte Kapitälchen umwandelen, Zeichen hoch- oder tiefstellen, alternative Zeichen aktivieren und vieles mehr. 
oder -sätze. So kann man zum Beispiel unterschiedliche Zahlenformate wie Versal- und Mediävalziffern in eine Schrift integrieren.

Die Arbeit eines Schriftgestalters umfasst heute also mehrere Schritte: die Zeichen der Schrift zu zeichnen, die Zurichtung der Schrift vorzunehmen, OpenType-Funktionen einzubetten, die Schrift in den geeigneten Formaten zu exportieren und gegebenenfalls ein Hinting der Schrift vorzunehmen. ${ }^{17}$

\section{DEMOKRATISIERUNG DES SCHRIFTENMARKTES}

Eines der ersten Programme, mit denen Fonts mittels Zeichen-Outlines und -Abständen digital definiert werden konnten, war Ikarus von der Firma URW++, welches jedoch mit einem Preis von über $\$ 100000^{18}$ nur für größere Firmen bezahlbar war. 1986 kam die Software Fontographer auf den Markt - zu einem Bruchteil des Preises, ${ }^{19}$ so dass auch Schriftgestalter ohne das entsprechende Kapital beginnen konnten, ihre eigenen Schriften zu produzieren. Dies führte zu einer Demokratisierung der Schriftgestaltung. ${ }^{20}$ Plötzlich entstanden viele neue Schriftenlabel und -Vertriebe auf einem Markt, der vorher nur von einigen wenigen Firmen bedient worden war. ${ }^{21}$ Die immer stärker werdende Verbreitung des Internets in

17 Vgl. http://en.wikipedia.org/wiki/Font_hinting (24.1.2013). Das Hinting einer Schrift gibt der Rendering-Engine des Betriebssystems Hinweise (Hints) wie die vektoriellen Outlines eines Buchstabens in Pixeln für die Bildschirmdarstellung umzusetzen ist. In den meisen Fällen wird das Hinting automatisch vom Schriftprogramm erstellt, doch kommt es hierbei oft zu Ungenauigkeiten. Für eine perfekte Bildschirmdarstellung muss die Schrift manuell Buchstabe für Buchstabe und Schriftgröße für Schriftgröße nachbearbeitet werden. Dadurch ist ein manuelles Hinting sehr aufwendig und teuer und wird nur selten gemacht, da die Displays immer besser werden (und sich die Notwendigkeit eines Hintings ab einer gewissen Auflösung erledigt haben wird) und das Druckergebnis einer Schrift vom Hinting nicht betroffen ist. 18 Vgl. http://en.wikipedia.org/wiki/Fontographer (20.12.2012).

19 Vgl. http://en.wikipedia.org/wiki/Fontographer (20.12.2012).

$20 \mathrm{Vgl}$. http://jyanet.com/cap/o03-1.htm (20.12.2012).

21 Anfang der 1990er Jahre war die Firma Linotype auf dem deutschen Markt als letztes tradionelles Schriftenhaus übrig geblieben, nachdem D.Stempel, Gebr. Klingspor, Bauersche Gießerei und Berthold von ihr aufgekauft, bzw. Konkurs gegangen waren. International waren nur Monotype und ITC nennenswerte Konkurrenten. 
den 1990er Jahren trug dazu bei, dass direkte Vertriebskanäle zwischen Produzent und Anwender entstanden. Damit sind jedoch nicht nur kommerzielle gemeint - sehr viele Schriften wurden kostenlos als Freefonts zur Verfügung gestellt. Solche Fonts sind oft gestalterische Experimente und in den seltensten Fällen zum Mengensatz geeignet. Häufig handelt es sich um so genannte Zierschriften.22

Vor der Einführung preiswerter Software zur Schriftengestaltung und dem Aufkommen des Internets war das Publizieren einer Schrift nur möglich, wenn einer der wenigen großen Schriftverlage einen Schriftentwurf für kommerziell verwertbar hielt und ihn unter großem Aufwand produzierte. Die Entwerfer gaben lediglich die sauber gezeichneten Buchstaben ab, die Digitalisierung und Zurichtung besorgte der Verlag. Dadurch nahmen die Verlage eine Stellung zwischen Schriftgestalter und Anwender ein, die nicht umgangen werden konnte. Gleichzeitig stellten Sie aber auch eine qualitätssichernde Instanz dar. Nachdem es durch preiswerte Schrifterstellungssoftware und das Internet als neuem Vertriebskanal prinzipiell jedem möglich war Schriften selbst zu erstellen und direkt an die Nutzer zu verkaufen oder zu verschenken, fiel diese Qualitätssicherung weg. Selbstverständlich wurde diese Entwicklung zwiespältig betrachtet. Während die einen einen Qualitätsverfall beklagten, sahen die anderen die Vorteile einer größeren Auswahl an Schriften, experimentelleren Designs und niedrigeren Kosten. Schnell setzte sich jedoch die Erkenntnis durch, dass die neue, ,unkontrollierte' Schriftenszene keine direkte Konkurrenz zu den etablierten Unternehmen, sondern eher eine Ergänzung darstellte. Während die etablierten Schriftenhäuser den Bereich der seriösen Typografie bedienten, lieferte die Szene der Selfmade-Schriftgestalter das Material für die in den 1990er Jahren populäre dekonstruktivistische Typografie ${ }^{23}$ und die sogenannte Grunge-Ästhetik. ${ }^{24}$

22 Im Englischen spricht man oft von display-fonts oder headline-fonts, um ihren dekorativen Charakter hervorzuheben und sie von Satzschriften für den Einsatz im Fließtext abzugrenzen.

23 Ihr populärster Vertreter war David Carson, bekannt für seine Gestaltung des Magazins Raygun und seien Bücher The End of Print (Schopfheim 1995) und The End of Print Bd. 2 (Schopfheim 1997). Mehr zur dekonstruktivistischen Typografie in: Roxane Jubert: Typography and Graphic Design. Paris 2006, S. 396-415, und Steven Heller und Véronique Vienne: 100 Ideen verändern Grafikdesign. Köln 2012, S. 192.

24 Mit ,Grunge-Ästhetik' ist das Bemühen gemeint, etwas kaputt oder verschlissen aussehen zu lassen. Man kann das Phänomen möglicherweise als 
Für Schriftgestalter stellte zu der damaligen Zeit der direkte Verkauf ihrer Schriften ein Problem dar. Um Bezahlungen erhalten zu können, musste man Verträge mit Kreditkartenunternehmen machen, was schwierig und teuer war. Zwei Unternehmen die 1999 gegründet wurden, hatten entscheidenden Einfluss auf die weitere Entwicklung. Das eine war das Online-Bezahlsystem PayPal. Es machte direkte Verträge mit den Kreditkartenunternehmen überflüssig, so dass viele Gestalter anfingen Schriften auf ihren eigenen Webseiten zum Verkauf anzubieten. Das andere wichtige Unternehmen war Myfonts. Es versteht sich nicht als Verlag, sondern als eine offene Verkaufsplattform für Schriften, die keine Wertung oder Auswahl vornimmt. Jede Schrift, die die rechtlichen und technischen Voraussetzungen erfüllt, kann zum Verkauf angeboten werden. Myfonts übernimmt den Verkauf und die digitale Auslieferung der Schriften, der Gestalter erhält einen Teil des Erlöses. Nach eigenen Angaben hat Myfonts aktuell ca. 103.00o Schriften von über 1100 Schriftgestaltern oder Foundries im Programm. ${ }^{25}$ Die Möglichkeit mit eigenen Schriften auch Geld verdienen zu können stellte für viele Gestalter einen Anreiz dar, qualitativ bessere Schriften anzubieten. Außerdem war die Zeit der ,wilden' Typografie abgeklungen, der Markt verlangte wieder nach seriöseren Entwürfen. Dadurch nahm das Angebot an Textschriften sprunghaft zu.

\section{TENDENZEN IN DER SCHRIFTGESTALTUNG}

Der Impuls eine neue Schrift zu gestalten, kommt heute in den seltensten Fällen von außen. Wenn ein Auftrag für eine Schriftgestaltung vergeben wird, so handelt es sich dabei in der Regel um eine Hausschrift für ein größeres Unternehmen. Eine unverwechselbare Schrift kann im grafischen Erscheinungsbild eines Unternehmens eine wichtige Rolle spielen und die Wiedererkennbarkeit und Prägnanz der Marke stärken. ${ }^{26}$

eine Gegenbewegung zu der durch Computer und Satzprogramme begünstigte Perfektionierung und Vereinfachung des Layoutens im Grafikdesign sehen. $25 \mathrm{Vgl}$. http://www.myfonts.com/about/ (Stand 20.12.2012). Es kommen jedoch jeden Tag mehrere neue Schriften hinzu.

26 Man denke nur an die Hausschrift von Mercedes-Benz. Auch wenn sie auf den ersten Blick recht gewöhnlich wirkt, so ist sie doch eigen genug, so dass eine Werbekampagne, in der lediglich Begriffe in der Hausschrift Corporate $S$ in Schwarz vor hellgrauem Grund standen, sofort als zu Mercedes gehörig verstanden wurde. 
Zum anderen stellt die Anfertigung einer Schrift - und die in diesem Fall meist daran geknüpfte Übertragung aller Nutzungsrechte - oft die kostengünstigere Variante zu einer umfangreichen Lizensierung einer existierenden Schrift dar.

In der Regel entstehen Schriften jedoch ohne Auftrag. Was die Gestalter dabei antreibt, kann ganz unterschiedlich sein - mal ist es die Neugier am Experiment oder die Weiterverwertung einer speziell für ein Projekt erstellten Schrift. Zum Teil sehen Schriftgestalter ihre Entwürfe auch als Lösung bestimmter typografischer Probleme. Manches Mal mag es die Spekulation auf wirtschaftlichen Erfolg sein, denn man kann gewisse Parallelen zum Musikmarkt sehen. Wie auch dort verkaufen sich einige wenige Schriften sehr oft - die absolut überwiegende Zahl der veröffentlichten Schriften fristet jedoch ein Schattendasein. Wie auch in der Musik, muss das jedoch kein Makel sein: Gerade im Grafikdesign wird oft nach Alternativen zum Mainstream gesucht, so dass auch selten gekaufte Schriften dankbare Kunden finden. Andererseits besteht immer die Möglichkeit, dass eine Schrift sich überraschend zu einem Verkaufsschlager entwickelt. ${ }^{27}$

$\mathrm{Ab}$ den 1990er Jahren gab es mehrere wichtige Strömungen in der Schriftgestaltung. Das eine waren Revivals historischer Schriften. Die etablierten Schriftenverlage hatten zwar historische Schriftentwürfe wie zum Beispiel Bembo oder Baskerville im Programm, doch waren ihre digitalen Versionen meist in den 1980er Jahren von den Fotosatz-Vorlagen abgenommen worden ${ }^{28}$ die wiederum von Drucken der Bleilettern abgenommen worden waren - vorzugsweise von höheren Schriftgraden, da diese größer waren und damit ein saubereres Bild lieferten. Damit ergaben sich zum Teil erhebliche Abweichungen zu den originalen Vorbildern aus dem 16. bis 18. Jahrhundert, die in den 1920er Jahren für die damaligen Nachschnitte der jeweiligen Schriften Pate gestanden hatten. ${ }^{29}$

27 In dem Fall winkt nicht nur finanzieller sondern auch ideeller Ertrag in Form von Anerkennung und Bekanntheit.

28 Vgl. Meggs, McKelvey (Hrsg.): Revival of the Fittest (wie Anm. 4), S. 50-53

29 In den 1920er Jahren gab es ein erstes Revival von Schriften aus dem 16. Jahrhundert. Stanley Morison veröffentlichte als Type-Director von Monotype 1929 zum Beispiel die Bembo, die sich an Drucken von Aldus Manutius aus dem Jahr 1495 orientierte. Natürlich war der Nachschnitt sehr viel homogener und sauberer als das Original, aber wie damals üblich, besaß jeder Schriftgrad eine für ihn spezifische Anpassung der Strichstärkenkontraste. Bei der Übertragung auf den Fotosatz wurden solche Drucke 
1495

BERNARDVS BEMBVS PATER. quin haccum ripae, tum arborum, tum.
etian fluminisamoenitate:neq; eft, quod

1929

When jobs have type sizes fixed quickly margins of error will widen unless all the determining calculations are based upon ABCDEFGHIJKLMNOPQRSTUVW

When jobs have type sizes fixed quickly

1990 margins of error will widen unless all the determining calculations are based upon ABCDEFGHIJKLMNOPQRSTUVW

5 Drei Stadien der Schrift Bembo. Oben: Die von Francesco Griffo 1495 geschnittene Schrift. Mitte: Die Bleisatzversion der Bembo von 1929, die Griffos Schrift zum Vorbild nahm. Unten: Die digitale Version der Bembo von 1990. Man sieht, dass die Schrift immer schärfer und kontrastreicher wurde.

Die neuen Revivals hatten unterschiedliche Zielrichtungen; manche versuchten das Gefühl von handgesetzten Bleilettern wiederzugeben, indem die Outlines amorph und nicht geometrisch sauber gezeichnet wurden, während andere versuchten klare und saubere Schriften zu zeichnen, deren Proportionen und Strichstärkenkontraste sich strenger an die Originale hielten (vgl. Abb. 6) ${ }^{30}$ Im Zuge dessen wurden auch die

abfotografiert oder die Formen direkt von den Lettern abgenommen, um daraus die Fotosatzschablonen herzustellen. Dabei wurden recht große Grade der Bleisatzschriften abgedruckt und fotografiert um eine maximale Qualität der Abbildung zu erhalten. Damit entstand eine Schrift in einem Kontrastverhältnis, das eigentlich nicht für Fließtexte gedacht war, sondern eher für Überschriften. Diese Schrift wurde dann digitalisiert, so dass der ,Fehler' mit übernommen wurde.

30 Vgl. Meggs, McKelvey (Hrsg.): Revival of the Fittest (wie Anm. 4), S. $154-159$. 
so genannten Designgrößen ,wiederentdeckt'. Das bedeutet, dass es von einer Schrift mehrere Varianten gibt, die sich vor allem in ihrem Strichstärkenkontrast unterscheiden und für bestimmte Schriftgrößen optimiert sind. Typische Größen sind (aufsteigend von klein nach groß): Caption, Text, Subhead und Display, wobei Caption den geringsten Kontrast und Display den größten hat.
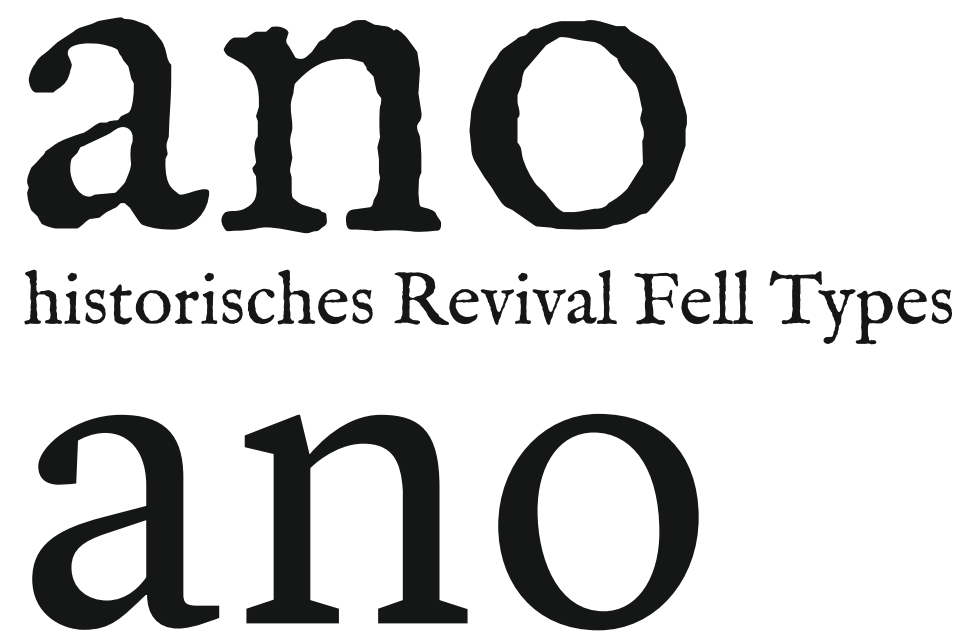

\section{historisches Revival Lyon Text}

6 Zwei unterschiedliche Ansätze mit Historischen Vorbildern umzugehen. Oben: ungeometrische, rauhe Outlines simulieren das Erscheinungsbild eines kleinen Buchstabens im Bleisatz. Unten: Das historische Vorbild wurde in klaren, vereinfachten, geometrischen Strichen nachgezeichnet und um geringfügige eigene Interpretationen ergänzt, wie die Tropfenserife beim a.

Nach den Revivals der Textschriften gab es eine große Zahl von Veröffentlichungen im Bereich der statischen Groteskschriften, deren bekannteste Vertreterin zweifelsohne die Helvetica ist. Dabei handelte es sich zum Teil um Schriften, die der Helvetica extrem ähnlich sehen, wie die Akkurat von Laurenz Brunner aus dem Jahre 2004 oder um Versuche, den ungelenken Charme früher Groteskschriften in zeitgemäß systematischere Bahnen zu lenken: u. a. FF Bau (2002), Theinhardt Grotesk (2009), Founders Grotesk (2010). Nach den statischen Grotesken gab es eine Reihe Veröffentlichungen, die den Bereich der geometrischen Groteskschriften ausloteten, wie zum Beispiel die sehr erfolgreiche Brandon Grotesque von Hannes van Döhren (2009), LL Brown (2011), PTL Superia (2011) oder 
Proxima Nova (2005), um nur einige zu nennen. Bei diesen wird zum Teil auch eine andere Strömung deutlich - der Versuch Zwischenräume zu besetzen. Manche der neuen Groteskschriften (z. T. auch unter dem Begriff Neo-Grotesque zusammengefasst), sind keine rein statischen oder rein geometrischen Schriften mehr wie ihre historischen Vorbilder. Vielmehr zeigen sich des öfteren dynamische Elemente, wie man sie von der Gill Sans (Eric Gill 1928-1930) kennt. Während dieser Weg der Fusion eher im Sinne einer Retro-Ästhetik zu sehen ist, gibt es es auch andere Strömungen, die aus der Idee der Fusion neue Formen schöpfen. Ein Beispiel stellen die so genannten Square-Sans-Schriften dar. Ihre an sich geometrischen Konturen stellen Buchstaben mit offenen Formen dar, wie sie für humanistische Groteskschriften typisch sind (vgl. Abb. 7). Einige der bekanntesten Vertreter sind die Klavika von Eric Olson (2004), Apex von Chester Jenkins (2003) oder FF Sanuk von Xavier Dupreé (2006).

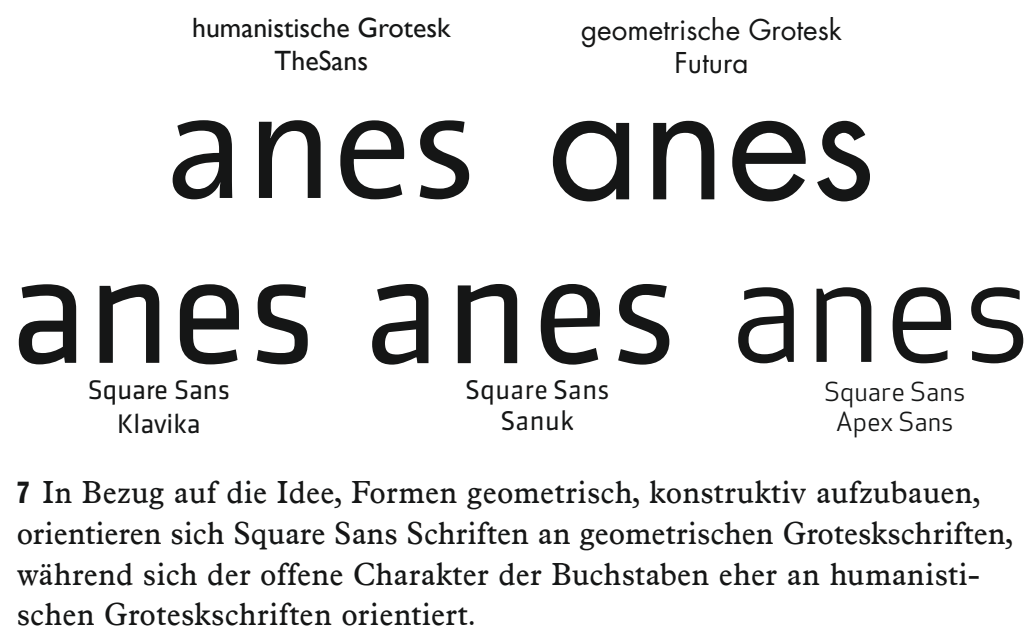

\section{AKTUELLE SCHRIFT-TECHNIK}

Die Programme, ${ }^{31}$ in denen heutzutage die meisten Schriftgestalter arbeiten, erlauben ihnen die wichtigsten Arbeitsschritte wie BuchstabenZeichnung, Zurichtung und OpenType-Features in einem geschlossenen

31 Nachdem Fontographer bis Anfang des neuen Jahrtausends das vorherrschende Programm war, übernahm Fontlab in der Folge die Marktführung, bekommt aber heute Konkurrenz von Programmen wie Glyphs und Robofont. 


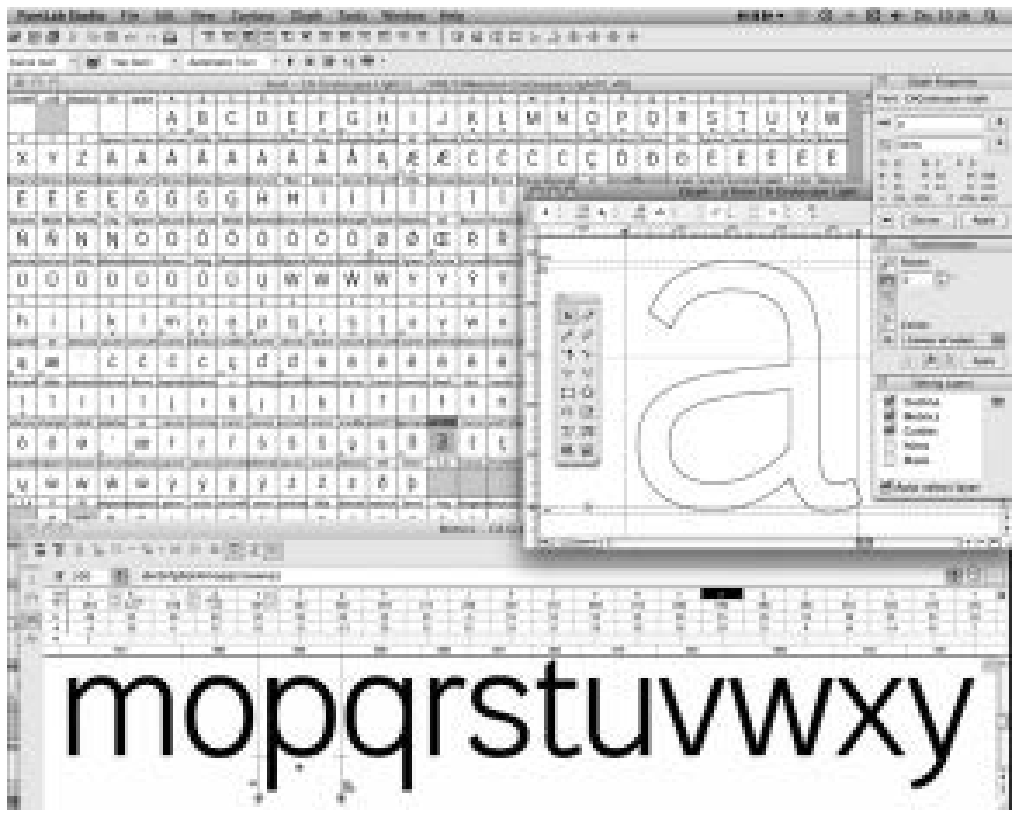

8 Arbeitsbereich von Fontlab mit der Glyphenpalette (alle Zeichen des Schriftsatzes) im Hintergrund, dem Zeichenbearbeitungsfester und dem Zurichtungsfenster (unten).

\begin{tabular}{|c|c|c|c|c|c|c|c|c|c|c|c|c|c|c|}
\hline \multirow{2}{*}{\multicolumn{2}{|c|}{$\frac{\mathrm{A}}{\mathrm{L}}$}} & \multicolumn{2}{|c|}{ B } & \multicolumn{2}{|c|}{ C } & D & B & F & $\mathrm{G}$ & H & \multicolumn{2}{|c|}{ I } & \multicolumn{2}{|c|}{$\mathrm{K}$} \\
\hline & & \multicolumn{2}{|c|}{ M } & \multicolumn{2}{|c|}{$\mathrm{N}$} & 0 & $\mathrm{p}$ & $Q$ & $\mathrm{R}$ & S & \multicolumn{2}{|c|}{$\mathrm{T}$} & \multicolumn{2}{|c|}{$\mathrm{U}$} \\
\hline 1 & 2 & 3 & 4 & 5 & 6 & \begin{tabular}{l|l}
7 & 8 \\
\end{tabular} & \begin{tabular}{l|l}
9 & 0
\end{tabular} & $-\mathrm{J}$ & $\mathrm{V}$ & W & $\mathrm{x}$ & $\mathrm{Y}$ & Z & $\&$ \\
\hline á & a & ì & $X$ & if & 3 & ft & 1 & b & u & " & 1. & . & $t$ & 5 \\
\hline e & $\mathrm{e}$ & e & $\ddot{\mathbf{c}}$ & Gi & f & \multirow{2}{*}{ t } & \multirow{2}{*}{ a } & \multirow{2}{*}{ I } & \begin{tabular}{l|l}
$x$ & $y$ \\
\end{tabular} & \begin{tabular}{l|l}
$z$ & 1 \\
\end{tabular} & 0 & [] & 1 & $?$ \\
\hline i & 1 & $i$ & i & $s$ & & & & & $v$ & $w$ & \multicolumn{2}{|c|}{ - } & : & ; \\
\hline$\delta$ & 8 & 8 & 0 & \multicolumn{2}{|c|}{ h } & \multirow{2}{*}{$\mathbf{m}$} & i & \multirow[b]{2}{*}{ a } & \multirow{2}{*}{0} & \begin{tabular}{l|l}
$\mathrm{R}^{2}$ & $\mathrm{q}$ \\
\end{tabular} & \multicolumn{2}{|c|}{. } & \multicolumn{2}{|c|}{ 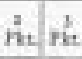 } \\
\hline ú & ù & ù & $\mathrm{U}$ & \multicolumn{2}{|c|}{1} & & $\begin{array}{l}\text { Fost: } \\
\text { Spuize. }\end{array}$ & & & P & \multicolumn{2}{|c|}{, } & Ger: & \\
\hline $\begin{array}{l}E \\
k\end{array}$ & EE & b & $k$ & ck & $\mathrm{c}$ & & & & $d$ & \begin{tabular}{l|l}
${ }^{2}$ & fin \\
\end{tabular} & A & $\mathrm{ft}$ & & \\
\hline $\begin{array}{l}\mathrm{d} \\
\alpha \\
\end{array}$ & $\begin{array}{l}\mathrm{c} \\
\mathrm{E} \\
\end{array}$ & c & b & b & & $\pi$ & nethes & c & d & $\mathrm{f} / \mathrm{ff}$ & $g$ & & $b \mathrm{~s}$ & \\
\hline
\end{tabular}

9 Plan eines Setzkastens für eine Antiquaschrift. 
Arbeitsumfeld auszuführen. Sie bieten ähnlich einem Setzkasten (vgl. Abb. 8 und 9) eine Übersicht über alle in der Schrift enthaltenen Zeichen. Jedes einzelne kann individuell bearbeitet werden. Dazu stehen dem Gestalter Zeichenwerkzeuge zur Verfügung, wie sie bei anderen vektorbasierten Zeichenprogrammen wie Illustrator ${ }^{32}$ üblich sind. Bei der Gestaltung einer neuen Schrift beginnt der Gestalter in der Regel nicht bei A und arbeitet sich bis $Z$ durch, sondern er wird versuchen, mit Buchstaben zu beginnen, die für die Schrift besonders prägend sind - entweder weil sie Formen aufweisen, die sich besonders oft wiederholen (wie Bögen und Einläufe beim $n$ oder Rundungen wie bei o) ${ }^{33}$ oder besonders ungewöhnlich sind und es wenig formale Entsprechungen im Alphabet gibt wie z. B. das zweigeschossige a, das s oder das dreigeschossige g. ${ }^{34}$ Dadurch dass diese Buchstaben nicht an sich wiederholende Formen gebunden sind, eröffnen sich dem Gestalter hierbei mehr Freiheiten, die er nutzen kann, um unkonventionelle Formlösungen anzubringen. Oft sind auch gerade diese Buchstaben diejenigen, anhand derer sich am leichtesten Schriften voneinander unterscheiden lassen.

Beginnt man mit einem $\mathrm{n}$, so lassen sich hiervon viele weitere Buchstaben direkt oder indirekt ableiten. Die Art, wie beim $\mathrm{n}$ der Bogen in den Stamm läuft (Einlauf) (vgl. Abb.10) und wie der Bogen (die Schulter) selbst beschaffen ist, sind Formmerkmale, die sich in der Schrift bei Buchstaben wie $h, m$ und $u$ wiederholen. Reduziert man ein $\mathrm{n}$ oder $\mathrm{h}$ auf den Stamm so kommt man zu Buchstaben wie i und 1 . Die Ausformung einer Rundung wie beim o beeinflusst wiederum das Aussehen von Buchstaben wie $\mathrm{c}$ und e. In der Kombination beeinflussen Stamm, Einlauf und Rundung das Aussehen von b, d, p, q. Sind diese Formen gefunden, so kann man erste Wörter setzten oder durch die Aneinanderreihung von Buchstaben den Rhythmus der Schrift beurteilen und gegebenenfalls korrigieren. Nun kann man die ungewöhnlicheren Buchstaben wie a, s und g gestalten. Die nächste größere Arbeitseinheit stellen Buchstaben mit Diagonalen dar. Sind auch diese gestaltet, so

$32 \mathrm{Vgl}$. http://www.adobe.com/de/products/illustrator.html (29.1.2013). Illustrator ist ein Zeichenprogramm, dass im Gegensatz zu einem Bildbearbeitungsprogramm wie Photoshop nicht auf die Bearbeitung von Pixeln sondern von Vektoren abzielt.

33 Vgl. Smeijers: Counterpunch (wie Anm. 2), S. 123.

34 Man spricht bei einem klassischen Antiqua-a von einem zweigeschossigen a, weil es zwei weiße Binnenräume hat. Im Gegensatz dazu hat ein kursives $a$ nur einen Weißraum und wird eingeschossig genannt. 


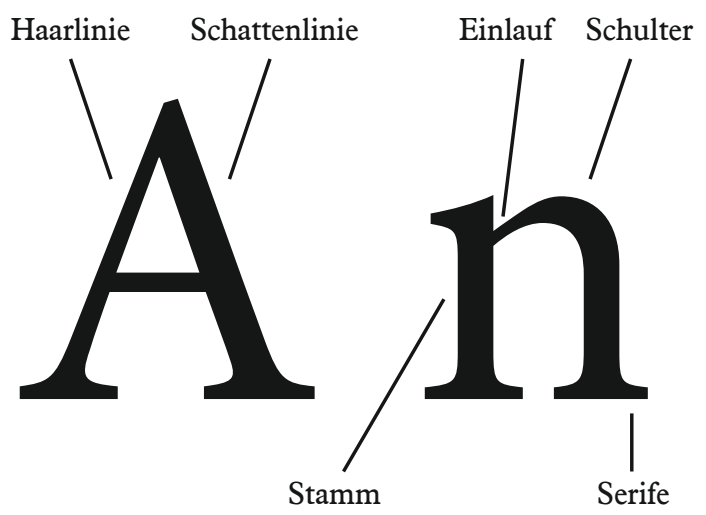

10 Die Namen einiger wichtiger Buchstabenteile.

werden nach und nach die letzten fehlenden Zeichen ergänzt. Selbstverständlich wird bei dieser Arbeit sehr viel von dem Prinzip des Copy \& Paste Gebrauch gemacht, doch stößt man hier schnell an Grenzen. In den seltensten Fällen kann man Elemente unmodifiziert von einem Buchstaben in den nächsten Übertragen. ${ }^{35}$ Fast immer müssen subtile Veränderungen vorgenommen werden, die den spezifischen Bedürfnissen des Buchstaben Rechnung tragen. Bei den Großbuchstaben wird ähnlich verfahren, wobei sie tendenziell etwas einfacher sind, da ihre Formen prinzipiell geometrischer sind.

Hat ein Gestalter einen kompletten Zeichensatz fertig, so folgt als nächstes die Zurichtung. Diese kann auf verschiedene Weise vorgenommen werden. Wichtig ist die Zuweisung von Vor- und Nachbreite für jeden einzelnen Buchstaben. Die am weitesten verbreitete Methode der Zurichtung basiert auf Überlegungen von Walter Tracy, ${ }^{36}$ wonach ähnliche Buchstabenformen auch ähnliche Weißräume erhalten sollten. Wenn also ein d auf der linken Seite eine bestimmte Vorbreite erhält, sollte ein q genau die gleiche haben. Hat man die Schrift nach der manuellen Methode einmal zugerichtet, und stellt fest, dass beispielsweise die Vorbreite von $\mathrm{d}$ nicht stimmt, so muss man diese Änderung nicht nur auf q sondern auch auf o, c, e und alle auf diesen Buchstaben basierenden

35 Am ehesten geht so etwas noch, wenn man man aus einem $\mathrm{n}$ ein $\mathrm{h}$ macht, indem man den Stamm nach oben verlängert, doch schon ein $\mathrm{m}$, das aus zwei n zusammengesetzt wird, wirkt optisch zu breit und muss schmaler gestaltet werden.

36 Vgl. Walter Tracy: Letters of Credit. Boston 1986, S. 70-8o. 
diakritischen Zeichen übertragen. Dies ist sehr aufwändig und es können leicht Fehler entstehen. Aus diesem Grund empfiehlt sich eine klassenbasierte Zurichtung, ${ }^{37}$ bei der man Buchstaben entsprechend ihrer jeweiligen linken oder rechten Ausformung in Klassen einteilt. Danach muss man nur noch jeder Klasse eine bestimmte Vor- oder Nachbreite zuweisen. Änderungen lassen sich dann leicht auf alle Buchstaben einer Klasse übertragen. Diese Methode der Zurichtung wurde erst in der digitalen Schriftproduktion möglich (und auch erst mit der Einführung von OpenType, weil erst dieses Format in der Lage ist, Klassen zu speichern) und macht es deutlich leichter, einen wesentlich höheren Grad an Perfektion in der Zurichtung einer Schrift zu erreichen, als es früher möglich gewesen wäre. Am stärksten werden jedoch die Vorteile der digitalen Schriftproduktion deutlich, wenn man sich die Unterschneidungen anschaut, die bei Blei- und Fotosatzschriften kaum zu realisieren waren. Wie schon erwähnt, erlauben es Unterschneidungen, auch noch die letzten unerwünschten Weißräume im Schriftbild zu eleminieren. Insofern kann man sagen, dass ein besonders homogenes Schriftbild ein Charakteristikum digitaler Schriften ist.

Interessanterweise führt maximale Homogenität jedoch nicht zu bestmöglicher Lesbarkeit. Ein gutes Beispiel dafür ist die GaramondVariante, in der die Wochenzeitung Die Zeit gesetzt ist. Ihr Schriftbild ist nicht völlig homogen, sondern weist immer wieder Lücken auf, die z. B. von Buchstaben wie $\mathrm{R}$ verursacht werden. Solche minimalen Irritationen halten das Auge des Lesers wach, und der Text gewinnt mehr Struktur, als wenn er einen völlig gleichmäßigen Grauwert hätte.

\section{EINFLUSS DER TECHNIK AUF DIE ÄSTHETIK}

Die Möglichkeiten der digitalen Schriftproduktion nehmen wie bereits angedeutet einen erheblichen Einfluss auf die Ästhetik der entstehenden Schriften. Eine nicht unerhebliche Rolle dürfte die Methode des Kopierens und Einfügens spielen. Sie macht den Arbeitsablauf schneller und

37 OpenType ist in der Lage Klassen in einem Font abzuspeichern. Eine Klasse beschreibt eine vom Gestalter definierte Gruppe von Zeichen. Im Falle der Zurichtung ist es sinnvoll Buchstaben die auf einer Seite gleich oder ähnlich ausgeformt sind in eine Klasse zu legen. Damit stellt man sicher, dass zum Beispiel c, ç, o, ö, ó, ò etc alle die gleiche Vorbreite kriegen, wenn sie alle in der gleichen Klasse ,links rund' liegen. 
effizienter, kann aber auch zu einer Reduzierung der Formenvielfalt führen. Wenn jeder Buchstabe individuell gezeichnet werden muss, so kann es viel leichter passieren, dass sich Buchstaben mit prinzipiell ähnlichen Formen dennoch in Details unterscheiden. Doch ist die Idee nicht neu, bestimmte Formen innerhalb einer Schrift mehrfach einzusetzen - immerhin trägt dies zu einer größeren Konsistenz im Schriftbild bei, was in der Regel ein wichtiger erwünschter Effekt ist. Schon bei der Produktion von Bleilettern wurden Punziereisen zum Erzeugen von BuchstabenInnenräumen für mehrere Buchstaben verwendet. ${ }^{38}$
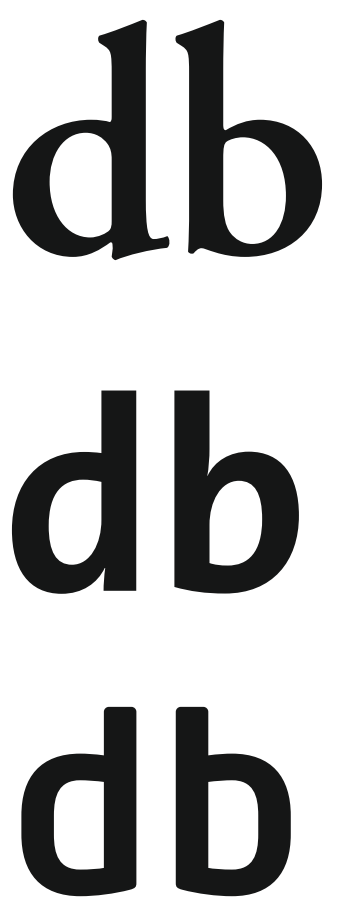
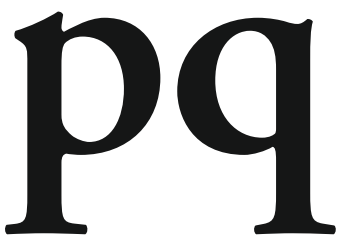

Adobe Garamond Pro

Semibold

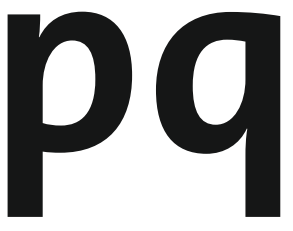

TheSans Bold

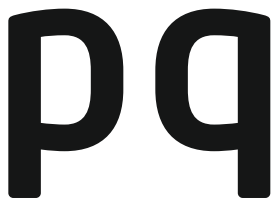

Neo Sans Bold

11 Bei der obersten Schrift sieht man, dass alle Buchstaben sehr individuell gestaltet sind - speziell im Bereich der An- und Abstriche und der Serifen. Bei der zweiten Schrift wurden mit minimalen Modifikationen jeweils die Buchstabenpaare $\mathrm{d}$ und $\mathrm{p}$, sowie $\mathrm{b}$ und $\mathrm{q}$ aus der gleichen Zeichnung erstellt. Im unteren Beispiel reichte dank Drehung und Spiegelung eine einzige Zeichnung für die Buchstaben $d, b$, p, und q.

38 Smeijers: Counterpunch (wie Anm. 2), S. 104. Smeijers zitiert Pierre Simon Fourniers Manuel Typografique von 1764 (S. 92). 
In der Abbildung 11 kann man sehen, wie eine Wiederverwendung von Formen stattfinden kann. Im Falle der TheSans wurden $\mathrm{d}$ und $\mathrm{b}$ jeweils gedreht (und minimal modifiziert), während bei der Neo Sans eine einzige Zeichnung für vier Buchstaben Pate stand.

Theoretisch sind die Möglichkeiten der Buchstaben-Gestaltung am Computer nicht begrenzt. Prinzipiell kann jede Form umgesetzt werden, es gibt lediglich gewisse praktische Aspekte die dazu führen, dass dennoch nicht alles Machbare auch gemacht wird. Die Schriftformate, die von den heutigen Betriebssystemen verarbeitet werden können, kennen keine farblichen Halbtöne - es gibt also in einem Buchstaben nur Schwarz und Weiß. ${ }^{39}$ Will man dennoch Zwischentöne erzeugen, so muss man dies über ein Halbtonraster ${ }^{40}$ machen. Die meisten Schriftenprogramme stellen als Arbeitsfläche für einen Buchstaben eine Fläche mit einer Größe von 1000 mal 1000 Einheiten zur Verfügung. Dies könnte man als die Auflösung eines Buchstabens bezeichnen. Man kann diese zwar vergrößern, doch kann das zu Komplikationen bei der Verwendung der Schrift im Betriebssystem führen. Um dies zu vermeiden, bleiben fast alle Gestalter bei der gewohnheitsmäßig etablierten Auflösung. Für ein gutes Halbtonraster ist dies jedoch eine sehr geringe Auflösung, so dass in der Regel von der Verwendung von Halbtönen abgesehen wird. Außerdem würde die Dateigröße der entstehenden Schrift so groß, dass auch dies erfahrungsgemäß zu Problemen bei der Verwendung der Schrift führt. ${ }^{41}$

Doch auch jenseits der Frage der Auflösung gibt es Schriftgestalter, die eine Einschränkung der Gestaltungsfreiheit beklagen, die sich aus der Verwendung von Vektoren als Formbeschreibung ergeben. Nicht jede per

39 Eine Ausnahme stellt Photofont dar. Die Firma Fontlab entwickelte ein Schriftformat, bei dem jeder einzelne Buchstabe ein Foto ist. Über Plug-ins können diese Schriften dann in einigen Programmen benutzt werden. Vgl. http://www.photofont.com.

40 Bei einem Halbtonraster werden Flächen die grau erscheinen sollen in Punkte mit regelmäßigen Abständen aufgelöst. Je nach Feinheit des Rasters und Betrachtungsabstand können die einzelnen Punkte nicht mehr wahrgenommen werden, so dass die Fläche einheitlich grau erscheint. Durch die Größe der Punkte lässt sich der Helligkeitseindruck steuern.

41 Obwohl heutige Drucker im Gegensatz zu älteren Druckern über genug Arbeitsspeicher verfügen müssten, führen Schriften mit zu vielen Vektoren (und jeder Rasterpunkt muss über vier Vektoren definiert werden) oft zu Abstürzen. 


\section{JEDER \\ JEDER $P Q$}

12 Beide Methoden, die Unschärfe einer gedruckten

Vorlage zu imitieren, liefern nur mangelhafte Resultate.

Links: Aufrasterung, rechts: Ungerade Konturen

Hand gezeichnete Linie lässt sich über Vektoren darstellen. ${ }^{42}$ Dies wird am deutlichsten bei Buchstaben mit einer rauen Kontur. Hier wirkt jede Umsetzung als Font bei genauerer Betrachtung unauthentisch.

In der Regel bestehen Satzschriften heute nicht mehr aus nur einem Schnitt. Als Minimalausbau gilt in der Regel ein Satz von vier Schriften: Regular, Italic, Bold und Bolditalic. Die meisten Satzschriften haben jedoch wesentlich mehr Schnitte. Während 1957 der stringente Ausbau der Univers auf zunächst 21 und später 27 Schnitte noch ein Alleinstellungsmerkmal darstellte, sind heute im professionellen Bereich gerade bei Grotesk-Schriften 12 Schnitte (Light, Regular, Medium, Semibold, Bold, Extrabold und das noch einmal in Kursiv) keine Seltenheit, ${ }^{43}$ was sich noch einmal multipliziert, wenn es die Schrift in mehreren Breiten gibt. ${ }^{44}$ Die Schriftsippe Thesis umfasst in der engeren Definition sogar 112 Schnitte. ${ }^{45}$ Durch das große Angebot wird die Vielfalt der Schnitte an

42 Vgl. Eduardo Berliner: Problems relating to the translation of a drawn letterform to a digital typeface. Reading 2003.

43 Bei serifenlosen Schriften ist das Phänomen des üppigen Ausbaus stärker zu beobachten, da Schnitte wie Light oder Thin bei Schriften mit Strichstärkenkontrast oft keine Sinn machen, da dieser verloren gehen würde.

44 Ebenfalls eine Eigenart, die vor allem bei serifenlosen Schriften anzutreffen ist.

45 Hinzu kommen noch Schnitte mit fester Zeichenbreite, so genannte Monospaced-Schnitte. Bei ihnen steht jedem Zeichen gleich viel Platz zur Verfügung. Dadurch stehen Zeichen, die in zwei Zeilen untereinander stehen exakt übereinander, was für Tabellensatz sehr praktisch sein kann. Andererseits ergeben sich im Schriftbild unharmonische DunkelheitsVerteilungen, da Buchstaben mit nur einem senkrechten Strich (wie ein i), auf dem gleichen Raum untergebracht werden müssen wie Buchstaben mit drei senkrechten (wie ein $\mathrm{m}$ ). Gerade diese Einschränkungen sehen aber Schriftgestalter immer wieder als eine Herausforderung an, und versuchen das Schriftbild im Rahmen der Möglichkeiten zu optimieren. 
sich schon zu einem Charakteristikum, das für einen Gestalter bei der Auswahl einer Schrift eine entscheidende Rolle spielen kann und dabei mit anderen Überlegungen wie zum Beispiel ästhetischen und finanziellen konkurriert. ${ }^{46}$

Möglich wird diese Form des Ausbaus durch die Interpolation, die ebenfalls ein für die digitale Schriftproduktion charakteristisches Merkmal darstellt. Hat man zwei Schriftschnitte gestaltet deren Gewichte ${ }^{47}$ möglichst weit auseinander liegen, so kann man theoretisch beliebig viele Zwischengewichte erstellen. Möglich sind sogar Extrapolationen, die wiederum neue Möglichkeiten in der Schriftproduktion ermöglichen. ${ }^{48}$ Darüber wie viele Zwischengewichte sinnvoll oder notwendig sind, gehen die Meinungen auseinander. So kann man einerseits die Ansicht vertreten, man solle den Anwender nicht überfordern und nur so viele Zwischenschnitte erzeugen wie nötig, und zwar so, dass die Gewichte sich klar voneinander unterscheiden. Auf der anderen Seite kann man dies für eine Bevormundung des Anwenders halten und sich auf den Standpunkt stellen, dass es besser sei, ihm möglichst viele Optionen zur Verfügung zu stellen. Aus Sicht der Schriftenvertriebe sind möglichst viele Schnitte wünschenswert, da diese die Option auf möglichst viele Verkäufe geben ${ }^{49}$ Vergleicht man heutige Schriftfamilien mit solchen aus der Zeit des Bleisatzes (z. B. Monotype Grotesque oder Trade Gothic ${ }^{50}$ ), so fällt auf, dass letztere wesentlich weniger konsistent waren. Die einzelnen Schriftschnitte sind viel individueller gestaltet. Dies liegt ganz offensichtlich an

46 Die Aspekte einer Schriftwahl sind vielfältig und werden gut dargestellt in: Stephanie und Ralf De Jong: Schriftwechsel. Mainz 2008, S. 88-112.

47 Mit Gewichten sind bei einer Schrift Abstufungen der Fettheit einer Schrift gemeint, also z. B. Light, Regular, Medium und Bold.

48 Vgl. Stefan Claudius: Jenseits der Interpolation. In: Slanted 19 (2012), S. 107-109.

49 Dies führt bei dem Label Fontshop dazu, dass manche Schriften in zwei Paketen angeboten werden, die jeweils die Hälfte aller verfügbaren Schnitte enthalten, das eine startet beispielsweise mit Light und das andere mit Thin. Vgl. http://www.fontshop.com/fonts/downloads/fontfont/ff_sanuk_ot_1 und http://www.fontshop.com/fonts/downloads/fontfont/ff_sanuk_ot_2.

50 Es ist bezeichnend, dass gerade zu solchen Schriften, die Pioniere im Bereich der Schriftfamilien waren, heute perfektere Überarbeitungen angeboten werden. Ausgerechnet in der Überarbeitung geht aber genau von dem, was den besonderen Charakter dieser Schriften ausmachte, viel verloren. Vgl. http://www.linotype.com/690o/classicgrotesque.html oder http://www.linotype.com/de/5737/tradegothicnext.html. 
Ursprüngliche digitale Version nach der BleiletternVorlage

\section{Hamburgefonstiv}

Trade Gothic Bold

\section{Hamburgefons}

Trade Gothic Bold No. 2

\section{Hamburgefonstiv}

Trade Gothic Condensed Bold No. 20

Überarbeitete Version 2008

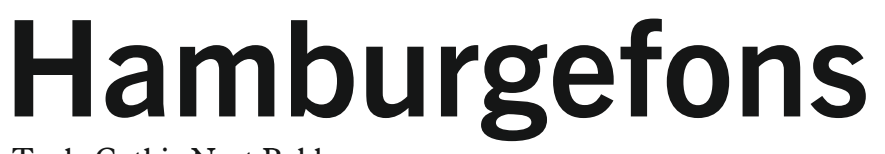

Trade Gothic Next Bold

\section{Hamburgefonstiv}

Trade Gothic Next Condensed Bold

13 Die unterschiedlichen Varianten der Trade Gothic in ihrer ursprünglichen Fassung und in der überarbeiteten Version von 2008

der heute praktizierten Interpolation, die zu sehr gleichmäßigen Veränderungen der Buchstaben-Dicken führt. Interessanterweise gilt heute ein Schriftenausbau nur dann als gut, wenn er besonders homogen ist. Das zeigt sich daran, dass von vielen Schriftenklassikern ,homogenisierte Versionen herausgebracht werden, in denen die ,Fehler' der Vergangenheit behoben werden. ${ }^{51}$ In der Grafik (vgl. Abb. 13) sieht man, dass die

51 Die Firma Linotype bringt unter dem Namenszusatz ,Next‘ Überarbeitungen erfolgreicher Schriften heraus, wie Avenir Next, Univers Next, Din Next, Trade Gothic Next etc. Vgl. z. B. http://www.linotype.com/de/90672/AvenirNextfamily.html (21.1.2013). 
ursprüngliche Trade Gothic über zwei unterschiedliche Versionen der Bold verfügte - eine mit runden und eine mit geraden Bögen - aber nur eine Condensed Bold, die dem Formprinzip der Bold folgte. In der Überarbeitung entschied sich Linotype, die Version Bold zu streichen und die Rundungen bei Buchstaben wie b, e und o an den Charakter der Trade Gothic Bold No. 2 anzugleichen. Die Rundungen wurden gegenüber der Vorlage runder gemacht und verlaufen nicht mehr in der Mitte senkrecht. Man kann dies als das Beheben eines Fehlers sehen, denn natürlich kann man argumentieren, dass Rundungen eigentlich rund sein sollten. Interessanterweise ist aber gerade der alte Schriftschnitt Bold Condensed No. 20 bei Gestaltern besonders beliebt. Speziell im kulturellen Bereich sieht man diese Schrift sehr oft.

Die Inkonsistenz einer Schrift, ihre Lebendigkeit, macht häufig einen großen Teil ihres Charakters aus. Schriften, die mit gewissen Fehlern, wie Inkonsequenzen in der Wiederholung von Buchstabenformen oder einer gewissen Unregelmäßigkeit des Schriftbildes behaftet sind, strahlen keine kühle Perfektion aus, sondern werden eher als ,warm wahrgenommen.

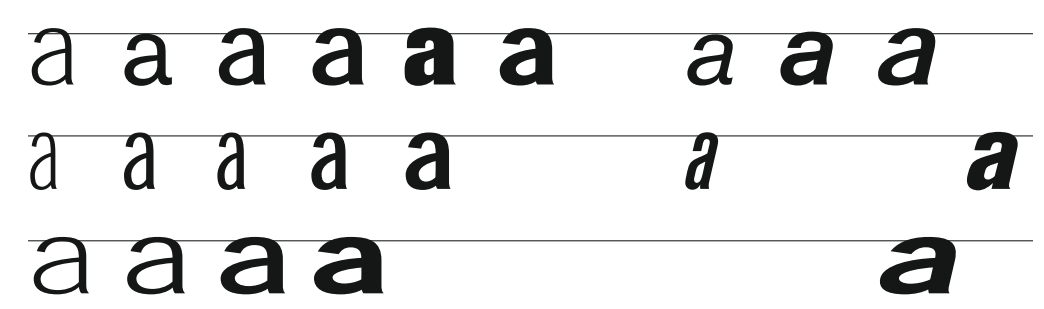

14 Die Grafik zeigt, wie Lückenhaft der Ausbau der Akzidenz Grotesk ist und wie starke Unterschiede das Design von Schnitt zu Schnitt aufweist.

Für genau diese Qualität werden viele klassische Schriften geschätzt, doch scheint zur Zeit kaum jemand interessiert daran zu sein, Schriften herzustellen, die versuchen eine ,warme' oder ,menschliche' Atmosphäre einzufangen. Wenn überhaupt wird dies über das Abrunden von Ecken versucht zu erreichen - wie in der sehr erfolgreichen Schrift Calibri.

Dass Abrundungen sich in letzter Zeit größerer Beliebtheit erfreuen, könnte daran liegen, dass es seit einiger Zeit eine Software namens Rounding UFO gibt, die es erlaubt per Knopfdruck Rundungen für eine komplette Schrift zu erzeugen, während vorher jede einzelne Ecke an 


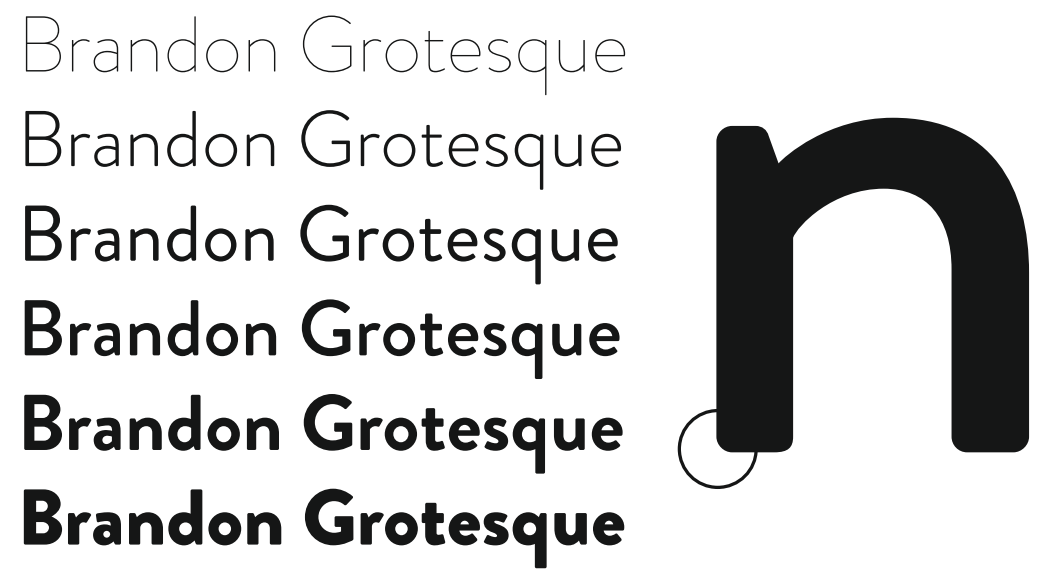

\section{Calibri Regular Calibri Bold Calibri Italic Calibri Bold Italic

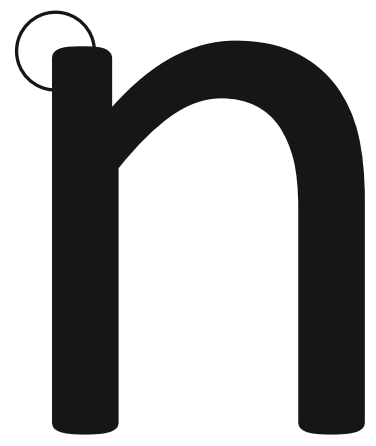

15 Die Calibri ist ein gutes Beispiel für einen Schriftentwurf, der als humanistische Groteske heute als sehr wenig auffällig wahrgenommen werden dürfte, durch die abgerundeten Ecken jedoch eine gewisse ,Wärme' bekommt.

jedem einzelnen Buchstaben per Hand bearbeitet werden musste - ein ausgesprochen aufwändiges Verfahren. Rounding UFO ist nur ein Beispiel für eine ganze Reihe von Hilfsprogrammen, die in den letzten Jahren auf dem Markt gekommen sind, mit denen man zwar keine kompletten Schriften generieren kann, die jedoch spezielle vorher sehr zeitaufwändige Arbeiten übernehmen können. Oft geht es darum, den Prozess der Interpolation zu erleichtern oder zu verbessern (z. B. Superpolator, Prepolator oder UFO Stretch). Diese externen Programme arbeiten mit einem speziellen Dateiformat, das sich UFO nennt und von den beiden Niederländern Just van Rossum und Erik van Blokland entwickelt wurde, die beide auch an der KABK in Den Haag im Rahmen des „Type and 
Media Master programs" unterrichten ${ }^{52}$ und die RoboFab ${ }^{53}$ entwickelt haben, eine Code-Bibliothek für die Programmiersprache Python, ${ }^{54}$ in der Befehle für das Speichern und Manipulieren von Schrift-Daten und das Dateiformat UFO (unified font object) gespeichert sind. Im „Type and Media"-Kurs gehört Programmieren zum Curriculum, da sie die Ansicht vertreten, dass der technische Aspekt der Schriftproduktion heute immer wichtiger wird, und erst die Unabhängigkeit von vorgegebenen Werkzeugen (in diesem Falle Schrifterstellungs-Programme) auch zu wirklicher gestalterischer Freiheit führt. Viele ihrer Schüler begeistern sich für das Thema Scripting (die Programmierung kleinerer Programme), verfolgen dies auch jenseits des Studiums weiter und veröffentlichen ihre Programme. So stehen dem Schriftgestalter heute viele Hilfsprogramme zur Verfügung, ohne die einige Aufgaben kaum noch zu bewältigen wären.

Diese Tendenz, Schriftgestaltung eher als eine Programmierleistung zu sehen ${ }^{55}$ steht im Gegensatz zu einer anderen Haltung, aus der heraus zum Beispiel das Programm Glyphs konzipiert wurde. Hier war die Maxime, dass der Benutzer sich nicht mit der Technik belasten solle, und möglichst viele Routineaufgaben von der Software im Hintergrund erledigt werden sollen. ${ }^{56}$ Beide Denkschulen haben ihre Berechtigung: Einerseits stimmt es, dass letztlich die Buchstabenformen und die Qualität der Zurichtung für eine Schrift entscheidend sind, doch ist es auch eine Tatsache, dass heute zur Erstellung einer Schrift wesentlich mehr gehört als nur das Zeichnen der Buchstaben. Eine Schrift ist in erster Linie ein Stück Software - ein Programm, das auf einem Computer läuft.

Trotz der enormen Menge an neu erscheinenden Schriften ist die Vorstellung, dass das Formenrepertoire in der Gestaltung von Buchstaben ausgeschöpft sei, wahrscheinlich so unzutreffend, wie sie es immer war. Dennoch werden die Unterschiede zwischen neu erscheinenden Schriften und den bereits existierenden immer subtiler. Neben den ästhetischen

52 Vgl. http://www.kabk.nl/pageEN.php?id=0016.

$53 \mathrm{Vgl}$. http://www.robofab.org.

54 Vgl. http://www.python.org/.

55 Das Programm Robofont wird damit beworben, dass es in erster Linie eine Schnittstelle darstellt, in die man eigene Skripte einbinden kann. Der tenor ist, dass der Gestalter seine eigenen Werkzeuge herstellen sollte, da diese den kreativen Prozess beeinflussen: „The tools you choose influence your creative process." (http://doc.robofont.com/ - 12.2.2013).

$56 \mathrm{Vgl}$. http://www.typografie.info/3/topic/27646-artikel-der-intuitivefonteditor-glyphs-ist-erschienen/ (12.2.2013). 
Kriterien werden Fragen nach dem Ausbau der Schrift immer wichtiger - also die Frage wieviele Schriftschnitte es gibt, was für (Sonder-)Zeichen in der Schrift enhalten sind und welche Sprachen sich mit ihr schreiben lassen. Ein weiterer wichtiger Aspekt ist die Lesbarkeit am Bildschirm. Dadurch, dass Browser nicht mehr auf die auf dem jeweiligen Computer installierten Schriften zurückgreifen müssen, sondern diese wie Bilder von einem Server laden können, entsteht ein großes Interesse an Schriften für die Nutzung im Netz. Das Problem besteht darin, dass das Lesegerät von sehr unterschiedlicher Qualität sein kann. Während ein normaler Computer-Monitor nur 72 Pixel pro Zoll darstellen kann, hat ein E-Paper bis zu 200 Pixel und ein Retina-Display, wie es im aktuellen iPad verbaut wird, sogar 326 Pixel pro Zoll. Damit liegt die Auflösung sogar über den gängigen Druckrastern, was zur Folge hat, dass über kurz oder lang die Frage nach der Lesbarkeit einer Schrift wieder die gleiche sein wird wie vor den Zeiten der Computerisierung. ${ }^{57}$

Ebenso bleiben Fragen nach ästhetischer Innovation von der Wahl der Mittel erstaunlich unbeeinflusst. So wenig wie es von der Hand zu weisen ist, dass die Werkzeuge die man nutzt, die Gestaltung beeinflussen, so richtig ist es, dass es Schriftentwürfe gibt, die heute noch Gültigkeit haben, obwohl sie mit den technischen Mitteln von $1722^{58}$ entstanden. Auch kann man durchaus sagen, dass aktuelle Schriftentwürfe auch mit den technischen Mitteln des 18. Jahrhunderts machbar gewesen wären. Schwer umsetzbar wäre dagegen der sehr homogene Ausbau von Schriftfamilien gewesen, da die Möglichkeit zur Interpolation fehlte, und auch das sehr ruhige Schriftbild das wir heute gewohnt sind wäre aufgrund der eingeschränkten Möglichkeiten, Unterschneidungen einzurichten, kaum erreichbar gewesen.

Spannend ist die Frage, wie kommende Schriftgestaltungsprogramm das Aussehen neuer Schriften beeinflussen werden. Ein wegweisender Ansatz ist Typegenerator ${ }^{59}$ von Remo Caminada und Ludovic Varone. Mit diesem Programmm zeichnet der Gestalter Pfade nicht mehr selbst, sondern er kontrolliert nur noch gewisse Gestaltungs-Parameter wie

57 In der Zwischenzeit haben jedoch einfache, offene Buchstabenformen Vorteile. Schaut man sich die Listen der am meisten verkauften Schriften von Linotype, FontShop und Myfonts an, so überwiegen die serifenlosen Schriften.

58 Die Schriftart Caslon entstand 1722, um nur ein Beispiel zu nennen.

59 Das Programm gewann einen wichtigen Designpreis, ist aber noch nicht auf dem Markt. Vgl. http://de.red-dot.org/2482.html (19.3.2013). 
Proportionen, Kontrast und Serifen. Die eigentlichen Buchstaben werden von der Software generiert. Damit ein solches Programm funktionieren kann, müssen sehr viele Designentscheidungen schon bei seiner Programmierung gefällt werden, was wiederum zu einer Einschränkung der zu erwartenden Resultate führt. Dennoch birgt ein solche Ansatz etwas Revolutionäres in sich. Die Schriftgestaltung wird dann nicht mehr Spezialisten vorbehalten sein, sondern jedem interessierten Anwender zugänglich sein.

\section{ABBILDUNGSVERZEICHNIS}

1 Fred Smeijers: Counterpunch (wie Anm. 2), S. 139.

2 Foto: Peter Reichardt.

5 Oben: Pietro Bembo: De Aetna, Aldus Manutius, Venedig 1495. Aus: James Sutton, Alan Bartram: An Atlas of Typeforms, 1988, S. 28. / Mitte: Monotype Type-Specimen. / Unten: Grafik des Autors.

9 Foto: www.druckhandwerk.de

3, 4, 6, 7, 8, 10, 11, 12, 13, 14, 15 Grafiken des Autors. 

CHRISTIAN STETTER

\section{BUCHSTABE UND ZEICHEN}

\section{DIE SCHRIFT: ZEICHEN DES ZEICHENS}

„Es sind also“, so eine bekannte deutsche Übersetzung des Beginns von Aristoteles' Peri hermeneias, „die Laute, zu denen die Stimme gebildet wird (ta en tê phōne ), Zeichen der in der Seele hervorgerufenen Vorstellungen (tōn en tē psychē pathèmatōn symbola), und die Schrift (ta graphomena) ist wieder ein Zeichen der Laute“" Diese Übersetzung, so prominent sie ist, ist falsch: Die bekannteste englische Ausgabe übersetzt das „ta en tē phōnē" richtig mit „words spoken“ und das „ta graphomena“ mit „written words" - was durch den Beginn des zweiten Kapitels der Schrift zweifelsfrei bestätigt wird. Dass einem ansonsten hochkompetenten Übersetzer ein solcher Lapsus unterläuft, zeigt, dass hier ein mächtiger Mythos am Werk war: die Auffassung, die Alphabetschrift sei eine Lautschrift. Auch diese Auffassung - so weit verbreitet sie auch ist - ist falsch: Zwar beruht das Prinzip dieses Schrifttypus auf der phonematischen Analyse einer oralen Sprache, des Altionischen, die etwa im 8. Jhd.v. Chr. ,fertig' ist, ${ }^{2}$ doch sie funktioniert nicht so. Vielmehr beruht ihr Funktionsprinzip auf einer abstrakten Kombinatorik: Dass nämlich für sich nichts bedeutende Elemente zu einem etwas bedeutenden Ausdruck zusammengefügt werden: zu einem Wort einer Schriftsprache. Denn Wörter sind die kleinsten

1 Aristoteles: Kategorien, Lehre vom Satz (Peri hermeneias). Übersetzt, mit einer Einleitung und erklärenden Anmerkungen versehen von Eugen Rolfes. Unveränderte Neuausgabe der 2. Auflage von 1925. Unveränderter Nachdruck. Hamburg 1974, S. 95.

2 Vgl. Rhys Carpenter: The Greek Alphabet Again. In: American Journal of Archeology 42 (1938), S. 58-69. Dt.: Noch einmal das griechische Alphabet. In: Gerhard Pfohl (Hrsg.): Das Alphabet. Entstehung und Entwicklung der griechischen Schrift. Darmstadt 1968, S. 84-105. 
in der Empirie, d.h. literalen Performanz begegnenden Zeichen einer jeden Schriftsprache.

Dies bedarf in verschiedenen Hinsichten der Erläuterung. Zum ersten: Buchstaben werden als Zeichen nur verwendet in einem speziellen schulischen Sprachspiel, in dem graphische Abbildungen von Buchstabenfiguren wie A, a, B, b usw. als Namen für etwas verwendet werden, das in demselben Sprachspiel als ,Laute' bezeichnet wird, aus dem die Wörter der oralen Sprache bestehen sollen, wiewohl zu den Voraussetzungen der Phonologie die Einsicht gehört, dass die Silbe die kleinste, nicht weiter segmentierbare Einheit der gesprochenen Sprache ist. ${ }^{3}$ Erst das macht ja aus dem Phonem die bedeutungsdifferenzierende Funktion, die es de facto in der klassischen Phonologie geworden ist. Genauer handelt es sich beim Phonem um eine abstrakte Relation, die eine Menge von phonologischen Oppositionen, etwa \{/a:/:/i:/ : /u:/\} auf eine Menge von Wörtern abbildet, die sich in ihrer Bedeutung systematisch unterscheiden: \{/da:/ :/di:/ : /du:/, ...\}, also nichts, das sinnlich wahrnehmbar wäre. Die auch unter Linguisten weitverbreitete Auffassung des Phonems als einer Spracheinheit verdankt sich offensichtlich einer Analogie, in der das als Buchstabenfolge geschriebene Wort als Modell für den Artikulationsmodus des gesprochenen genommen wurde und weithin bis heute wird: das Grundmodell für das, was seit den 9oer Jahren des 20. Jahrhunderts als ,Skriptizismus' beschrieben worden ist. ${ }^{4}$

Zweitens: Als die kleinsten ,bedeutungstragenden' Einheiten einer Sprache gelten der systematischen Linguistik die Morpheme, lexikalische Morpheme wie sag-, in, baum-, ..., grammatische Morpheme wie -e, $-s t,-t, \ldots$ Doch diese sind konzipiert als Systemeinheiten: ${ }^{5}$ theoretische Konstrukte, die der Beschreibung und Erklärung der Wortbildung als eines sprachsystematischen Prozesses dienen. Die kleinsten Einheiten der aktualen Rede sind in jeder menschlichen Sprache die Wörter. Auch

3 Vgl. Roman Jakobson: Kindersprache, Aphasie und allgemeine Lautgesetze. Frankfurt a. M. 1969.

4 Vgl. Christian Stetter: Schrift und Sprache. Frankfurt a. M. 1997; Sybille Krämer: Das Medium als Spur und Apparat. In: dies.: Medien, Computer, Realität: Wirklichkeitsvorstellungen und neue Medien. Frankfurt a. M. 1998, S. 73-94, und Christa Dürscheid: Einführung in die Schriftlinguistik. 3., überarbeitete und ergänzte Aufl. Göttingen 2006.

5 Vgl. André Martinet: Grundzüge der Allgemeinen Sprachwissenschaft. Autorisierte, vom Verf. durchgesehene Übersetzung aus dem Französischen von Anna Fuchs, unter Mitarbeit von Hans Heinrich Lieb. Stuttgart 1963. 
das bedarf keiner weiteren Erläuterung, das Faktum wird durch die Evolution eines Registers der Alphabetschrift bestätigt: der Getrennt- bzw. Zusammenschreibung von Wörtern, die sich ab dem 2. vorchristlichen Jahrhundert entwickelt. Dieses Register belegt augenfällig, wie das Wort als Grundeinheit der Sprache im Bewusstsein der Schriftkundigen Gestalt annimmt.

Das Funktionsprinzip der Alphabetschrift beruht also auf einer abstrakten Kombinatorik, die analog der subsemantischen Artikulation der oralen Sprache verfährt. Es gilt heute als das universelle Funktionsprinzip von Schrift überhaupt. Beleg dafür ist das Schriftsystem des Japanischen. Es umfasst neben dem welthistorischen Antipoden der Alphabetschrift, den Hanzì, im Japanischen Kanji, nicht nur eine aus diesen abgeleitete Silbenschrift, die beiden Register der Kana, die sich zwar figürlich unterscheiden, aber in ihrem Wertebestand identisch sind: die kursiven Hiragana und die Katakana, die aus Fragmenten von Kanji bestehen. Heute ist dieses Schriftsystem durch ein weiteres Register ergänzt: die Romaji, das lateinische Alphabet, das - wie in China auch - zur Transkription insbesondere von Namen verwendet wird. Die offensichtlich gelungene ,Kohabitation' dieser verschiedenen Schriftregister in einem umfassenden Schriftsystem ist wohl eines der faszinierendsten Phänomene in der Evolution von Schriften, kulturhistorisch ebenso bedeutsam wie linguistisch oder soziologisch.

Schließlich und endlich: Zeichen sind für den Menschen - wenigstens im normalen Sprachgebrauch - externe visuelle oder akustische oder auch taktil erfassbare Erscheinungen der Umwelt, die wir kognitiv erfassen und mittels unserer Sprachkompetenz auf unser Wissen abbilden. Die durch Spatien getrennten Buchstabenfolgen eines Textes - selbst eine isolierte Inskription wie ,Eingang - werden für uns lesbar und verständlich, indem wir sie auf unser internes Sprachwissen abbilden, sie eben mittels dieser, entziffern'. Damit erst werden diese Erscheinungen zu unserem Wissen - und damit sind sie, in strengem Sinn, für uns keine Zeichen mehr, sondern Wissensbestand.

\section{DER MYTHOS DER REPRÄSENTATION}

Universell scheint das Funktionsprinzip der Alphabetschrift insbesondere aus dem Grund zu sein, weil es auch in einem weiteren Sinn auf einem abstrakten Prinzip beruht: Jedes Wort einer Alphabetschrift stellt stets und allein die Form dieses Wortes dar, nicht seine Bedeutung. Bestätigt wird 
dies gerade durch eine Schriftsprache wie das Englische, das durch eine ,tiefe' Orthographie gekennzeichnet ist: Man kann der Buchstabenfolge eines Wortes nur selten die orale Lesart entnehmen. Hat man jedoch ein Grundrepertoire von Wörtern einmal gelernt, so wird man auch in dieser Sprache viele Texte lesen und vorlesen können, ohne indessen die Bedeutung aller Wörter zu verstehen. Ja es kann vorkommen, dass man etwa einen Fachtext Wort für Wort zu lesen imstande ist, ohne jedoch seinen Sinn zu begreifen. Die Bedeutung eines Wortes lernt man, indem man erstens seine syntaktische Verwendung erlernt. Zweitens, welche Bezugnahmegebiete mit den kategorematischen Wörtern eröffnet werden, den Nomina, Verben und Adjektiven, und worauf mit ihnen Bezug genommen wird. Darauf gibt eine wie auch immer geartete Buchstabenfolge nie Hinweise - im Gegensatz zu den sogenannten ursprünglichen Bildern des Chinesischen wie 人, 女, 手, 曰, 月, ..., Mensch, Frau, Hand, Sonne, Mond, die zumindest in der Frühphase der Entwicklung dieser Schrift wohl als Piktogramme, schematisierte bildliche Darstellung von Lebewesen, Gegenständen und anderem gedient haben mochten. Die chinesische Schrift ist daher aus europäischem Blickwinkel traditionell als Ideographie begriffen worden, als eine Art Begriffsschrift - eine Auffassung, die zurecht kritisiert und korrigiert worden ist. ${ }^{6}$ Der linguistisch entscheidende Punkt dieser Debatte ist der folgende: Ein Hanzì, gleich ob einfaches oder komplexes Zeichen, stellte offenbar schon in der Frühphase der Entwicklung dieser Schrift stets ein Wort der chinesischen Sprache dar, so wie jede durch zwei Spatien begrenzte Buchstabenfolge in einem deutschen, englischen, französischen, spanischen Text stets ein Wort der betreffenden Sprache darstellt - und dieses, darstellt ${ }^{t}$ ist hier nicht im repräsentativen Sinn zu lesen, sondern im exemplikativen: Jede entsprechende Buchstabenfolge etwa auf dieser Seite dieses Textes exemplifiziert, ist also ein Muster für das, was ein Wort der deutschen Schriftsprache ist. Auch die Hanzì sind somit eine Logographie, ebenso wie das ,Mischsystem ' aus Kanji und Hiragana - keine sonderliche Erkenntnis, denn dies gilt, cum grano salis, für jedes traditionell gewachsene Schriftsystem dieser Welt.

Dies macht - nebenbei - deutlich, wie irreführend der Topos des Repräsentativen ist, mit dem die Schrifttheorie geradezu kontaminiert ist. Wo wir ein Schriftverfahren verwenden, um Einheiten der oralen Sprache darzustellen, verwenden wir stets besondere Notationen, um anzudeuten,

6 Vgl. John DeFrancis: Visible Speech: The Diverse Oneness of Writing Systems. Honolulu 1989, und Geoffrey Sampson: Chinese Script and the Diversity of Writing Systems. Linguistics 32 (1994), S. 117-132. 
dass hier die Schriftelemente nicht in ihrer genuinen Funktion verwendet werden: nämlich um andere, gegenwärtig oft nicht präsente Personen zu grüßen, etwas zu berichten oder über etwas zu informieren usw., insbesondere dann, wenn das Mitgeteilte verlässlich oder nachprüfbar sein sollte. Also in einem Modus, der nicht der intersubjektiven Kommunikation dient, sondern der Dokumentation. Dies zeigen schon die Lautumschriften in fremdsprachigen Wörterbüchern. Das orale Wort, das wir als, weiß schreiben, könnte man etwa als /vaes/ darstellen, ein Graphismus, der jedoch viele dialektale Varianten nicht erfasst, die im Deutschen von /ve:s/ bis /voas/ reichen. Also wiederum eine skriptizistische Weise der Darstellung, die einen bereits schriftlich geregelten Normaldialekt voraussetzt, dessen Extension weit über die regionale Verbreitung oraler Dialekte hinausgeht, im Grunde genommen alle Orte auf dieser Erde umfasst, wo er verwendet wird oder wo deutschsprachige Bücher in einer Bibliothek sehen, und sei dies auch in einem japanischen Seminar. Dies schon deswegen, weil schriftliche Texte die Phänomeneigenschaft haben, zeitlich pertinent, dauerhaft zu sein, die orale Äußerung dagegen ephemer, vorübergehend. Die Geschichte gleicht somit sehr der von Hase und Igel. Das Repräsentationsmodell ist, wie diese Überlegungen zeigen, logisch zirkulär. Der Grund dafür muss in einem undurchschauten logischen Verhältnis zwischen oraler und Schriftsprache liegen, das von diesem Schriftmodell nicht erfasst wird. Wie ist also dieses Verhältnis logisch widerspruchsfrei zu denken?

\section{NOTATIONALE EIGENSCHAFTEN UND TYPENKONZEPTE}

Um dieses Problem zu lösen, muss man die unterschiedlichen logischen, d. h. die notationalen Eigenschaften von oraler Sprache und Schrift betrachten: Jedes alphabetschriftliche Wort ist darstellbar in einer logischen ,Normalform', die geradezu ideal durch ein mit einem Schreibprogramm erzeugtes Wort repräsentiert wird - wie etwa die Wörter dieses Textes. Denn die Erzeugung jeder Buchstabenfigur ist in einem Teilprogramm $P_{i}$ eindeutig beschrieben, das verschieden ist von jedem Programm zur Erzeugung eines anderen Buchstaben. Dieses Programm $P_{i}-$ z. B. $P_{y}$, das Programm zur Erzeugung der Minuskel y, die Zuweisung des Formats sei hier ausgeklammert - kann man als den Typen y auffassen: die - in philosophiehistorischen Termen - realistische Version des Typenbegriffs: Der Typ gilt als das Urbild aller Kopien, die einander nicht nur ähnlich, sondern gleich sind. Dagegen sind die Tokens eines nominalistischen 
Typen-Konzepts als Kopien eines wie auch immer beschaffenen Musters begriffen, die einander nur mehr oder weniger ähnlich, nie aber exakt gleich sind. Prototyp eines solchen Typenkonzepts ist das orale Wort: Es existiert stets und nur als Menge von dialektal mehr oder weniger verschiedenen Kopien, die einander kaum einmal exakt gleichen, selbst nicht in den Artikulationen derselben Person, denn die können ruhig oder erregt, laut oder leise, deutlich oder undeutlich gesprochen sein.

Nelson Goodman hat die technischen Mittel geschaffen, den wesentlichen Unterschied logisch zu fassen, auf dem diese Sachlage beruht ${ }^{7}$ : Jedes Wort eines alphabetschriftlichen Textes, etwa dieses Satzes, besteht aus einer Folge von Figuren - J, e, d, s, W, ... - deren jede wiederum qua Buchstabe Element einer Menge von Figuren $\operatorname{MBF}\{A, a, B, b, \ldots, Z, z$, ...) ist. Diese wird von Sprache zu Sprache leicht variieren, insbesondere aufgrund von Sonderzeichen wie $\tilde{\mathrm{n}}$, ç, å, ... Doch solange ein deutscher oder spanischer oder französischer oder ... Text hinreichend deutlich geschrieben ist, wird man jede Figur als Element von MBF identifizieren und von Tintenkleksen, Fliegendreck oder anderen Erscheinungen in der betreffenden Inskription unterscheiden können. Jede so identifizierte Figur ist somit eindeutig einem bestimmten Figurentyp zugewiesen, d.h. ,effektiv differenziert'. Ist ein ganzes Inskriptionsschema, etwa ein Wort dieses Textes, durchgängig effektiv differenziert, dann ist es logisch digital. Dasselbe gilt aus den genannten Gründen für diesen ganzen Text. Die logische Eigenschaft der Digitalität einer Inskription, sei diese ein einzelnes Wort oder ein ganzer Text, ist die Voraussetzung dafür, ihn auch technisch digital erzeugen zu können. Nehmen wir dagegen ein Bild wie Klees Schwarzer Fürst, so ist kein ,Element' dieses Bildes, etwa die Striche, die in diesem Bild als Nase oder Lippen der dargestellten Figur ,gelesen ${ }^{6}$ werden, als Element eindeutig einem Strich-Typ zuzuordnen. Jede noch so genaue Kopie dieses Bildes wird in jedem Detail in irgendeiner Nuance vom Original abweichen. Es ist durchgängig nicht effektiv differenziert und damit logisch analog.

In einer Alphabetschrift mit einer hinreichend ,gefestigten' Normorthographie - im Deutschen beginnen sich allmählich die Turbulenzen zu legen, die die sogenannte Rechtschreibreform von 1995 erzeugt hat lässt sich somit jedes Wort der betreffenden Sprache durch genau eine Buchstabenfolge digital darstellen. Eben dies macht die Entstehung

7 Vgl. Nelson Goodman: Languages of Art. An Approach to a Theory of Symbols (1968). Indianapolis, New York 1997. 
exakter Homographen wie ,weiß‘, ,Kiefer' usw. möglich. Dem literalen Wort des Deutschen <weiß> lässt sich dann eine Menge oraler dialektaler Varianten zuordnen wie etwa /vaes/, /ve:s/, /voas/ usw. Keineswegs repräsentiert also ein schriftliches Wort ein orales: Vielmehr handelt es sich um eine Abbildung einer insgesamt unüberschaubaren Menge oraler Varianten auf genau eine, weil digitale literale Wortform. Denn allein diese digitale Darstellung, die nicht die Repräsentation eines - wie auch immer gedachten - oralen Wortes ist, sondern die Exemplifikation eines literalen Musters, ermöglicht die Konstruktion eines Schemas, in dem per se jedem Wort der betreffenden literalen Sprache sein exakter Ort in einem zweidimensionalen, alphabetisch doppelt geordneten Schema zugewiesen ist: dem Wörterbuch. Das Verhältnis von oraler und Schriftsprache lässt sich also - wie oben schon angedeutet - nur extensional fassen, als Abbildung einer unfassbaren Menge von dialektalen Varianten auf ein digital organisiertes, alphanumerisch geordnetes Wörterbuch. Erst diese Abbildung macht es ja möglich, eine Variante von $\mathrm{x}$ als Variante von $x$ identifizieren.

Der Extensionsbereich eines oralen Dialekts - und sei dies auch einer von Sprecherziehern gern als ,Hochsprache' bezeichneten Verkehrssprache wie des oralen ,Hochdeutschen' - ist eben zu jedem Augenblick der und nur der, wo auf der Welt eben dieser Dialekt gerade gesprochen wird, und dies ändert sich von Tag zu Tag, wenn auch statistisch relevant nur in begrenzten Regionen. Der Extensionsbereich einer Schriftsprache, sei dies nun Deutsch oder Englisch oder Chinesisch usw., umfasst dagegen alle Gebiete, wo die betreffende Schriftsprache mehr oder weniger dauerhaft verwendet wird. Allein der pertinente Extensionsbereich der englischen Schriftsprache - in welchen Varianten auch immer - dürfte heute den gesamten Globus umfassen. ${ }^{8}$

\section{DIE DEFRANCIS-THESE}

Jede Alphabetschrift ist also - zumindest in ihrem Kernbestand an Buchstabenfiguren, orthographischen Zeichen usw. - logisch digital. Wie verhält es sich nun mit ihrem großen Antipoden, den Hanzì, den

8 Hierzu wäre allerdings anzumerken, dass mit dem Internet alle diese Überlegungen auf dem Prüfstand stehen, denn damit ist potentiell jede Nachricht in jeder beliebigen Schriftsprache universell verbreitbar bzw. zugänglich. Dies zeigt, dass die Zeiten von ,Sprachgeographie‘ definitiv vorüber sind. 
chinesischen Zeichen? Der Sinologe John DeFrancis hat in einem vieldiskutierten Buch nicht nur die verbreitete Meinung widerlegt, die chinesische Schrift sei eine Art Begriffsschrift in dem von Leibniz beschriebenen Sinn einer ars characteristica: dass nämlich ihre Schriftzeichen direkt die Bedeutung des betreffenden Hanzì wiedergeben, logisch gesprochen also die Intension, den Sinn des Zeichens. Vielmehr seien die Hanzì eine vollgültige Schrift in linguistischem Sinne: Jedes Zeichen repräsentiere ein Wort der chinesischen Sprache, die Hanzì seien also eine vollentwickelte Logographie - so wie ja auch jede moderne Alphabetschrift eine Logographie ist. ${ }^{9}$ Darüber hinaus hat er die These vertreten, die chinesische Schrift beruhe wie jede andere Schrift auch auf einem phonetischen Prinzip..$^{10}$ Dies ist von Geoffrey Sampson partiell bestätigt, als generelle These jedoch in Frage gestellt, wenn nicht widerlegt worden. ${ }^{11}$ Er teilt mit DeFrancis die Datenbasis: Etwa 90\% aller Hanzì, deren Gesamtzahl auf über 90.000 geschätzt wird, sind komplexe Zeichen, die ihrerseits je aus zwei Hanzì bestehen, einem sogenannten ,Signifikum', das einen Hinweis auf die Bedeutung des Gesamtzeichens gibt, und einem zweiten Zeichen, dem ,Phonetikum', das die orale Lesart des Zeichens andeutet. Grundlage dafür sind zwei das orale Chinesische charakterisierende Fakten: Fast sämtliche Wörter dieser Sprache sind Einsilber, und deren Anzahl ist entsprechend klein: Durchschnittlich entsprechen einer Silbe acht verschiedene Bedeutungen. Homonymie ist gleichsam der Normalfall im oralen Chinesisch. Auf diesem Faktum beruht die Evolution der komplexen Hanzì. Allerdings handelt es sich hier um einen weiten Begriff von Homonymie. Die Aussprache der betreffenden Wörter ist in den meisten Fällen nicht genau gleich, sondern einander ,nur ähnlich. Dies nutzt das System der Hanzì aus: Das Phonetikum eröffnet sozusagen einen Spielraum von möglichen oralen Lesungen des Zeichens, und dieser wird durch das Signifikum eingegrenzt. So besteht das Zeichen mit der Bedeutung ,Mutter, 妈, gesprochen ,mā', d. h. im sogenannten ersten Ton, aus dem Phonetikum 马, má' (Pferd), gesprochen im dritten Ton, und dem Signifikum, 女“ (Frau). Die Töne haben bedeutungsdifferenzierende Funktion. Beide Verfahren beruhen - wie unmittelbar zu sehen ist - auf Analogie. Das System der Hanzì ist so von der Form- wie von der Bedeutungsseite her betrachtet ein logisch analoges System. Natürlich lässt sich heute für jedes Hanzì eine digitale technische Normalform erzeugen,

9 Vgl. Anm. 2.

10 Vgl. DeFrancis: Visible Speech (wie Anm. 6).

11 Vgl. Sampson: Chinese Script (wie Anm. 6). 
der ihrerseits verschiedene Formate zugewiesen werden können. Aber das digitale Gesamtsystem lässt sich nicht in ein alphanumerisches System übertragen, das für Menschen lesbar wäre. Daher auch die verschiedenen Ordnungssysteme für Hanzì- oder Kanji-Wörterbücher, die traditionell auf den sogenannten Radikalen beruhen. Die Mengen der Striche, Radikale und der Hanzì bzw. Kanji überschneiden sich jedoch: Logisch sind diese Systeme daher nicht in einen digitalen Zustand zu überführen.

Diese Fakten bilden insgesamt die Basis für Sampsons Argument gegen DeFrancis: Er räumt ein, dass in den ersten Phasen der Entwicklung der Hanzì dank der Funktion des Phonetikums dieses Schriftsystem in der Tat auf einer phonetischen Basis beruht habe. Doch aufgrund der Lautwandelprozesse, die die Evolution des oralen Chinesischen als einer ,natürlichen' Sprache ebenso charakterisieren wie die jeder andere orale Sprache auch, sei dieser phonetische Grundcharakter des Systems der Hanzì mehr und mehr verschwunden und existiere heute de facto nicht mehr. Daher charakterisiert Sampson das heutige System der Hanzì als eine ,Glottographie' in dem Sinne, dass es eben ein vollgültiges Sprachsystem sei, und in näherem Sinn als eine ,Logographie', eine WortSchrift - eine Kategorisierung, die zweifellos zutrifft, aber eben nicht spezifisch ist. Denn jedes alphabetschriftliche orthographische System, das über das Register der Getrennt- und Zusammenschreibung verfügt, ist trivialerweise eine Logographie, denn es trennt eben die Wörter durch Spatien. Jeder Text einer solchen Schriftsprache definiert somit diese rekursiv erneut als eine solche. Nur so konnte etwa mit der Differenz der Schreibungen ,sogenannt' und ,so genannt' ein Bedeutungsunterschied verbunden werden.

Philosophisch ist Sampsons Argument indessen hochbedeutend, gerade weil es die logisch analoge Funktionsweise des chinesischen Schriftsystems so klar herausarbeitet, auch wenn Sampson selbst nicht diese Kategorisierung verwendet. Denn wenn wir mit W. von Humboldt oder Wittgenstein die Sprache als das Medium menschlichen Denkens begreifen, dann muss dies auch für die logischen medialen Eigenschaften von Schriftsystemen gelten. Medien sind der ,Botschaft' gegenüber, die sie vermitteln, nie neutral: Sie formen sie. ${ }^{12}$ Dass formales Denken intern mit der logisch digitalen Natur der Alphabetschrift zusammenhängt, demonstriert schon die dialektische Übung in Platons Parmenides. ${ }^{13}$

12 Vgl. Krämer: Das Medium als Spur und Apparat (wie Anm.4). 13 Vgl. Stetter: Schrift und Sprache (wie Anm. 4), S. 331-355. 


\section{DIE AUTONOMIE-THESE}

DeFrancis wie Sampson verwenden in ihrer Argumentation den Begriff ,phonetisch' in einem weiten, vom üblichen linguistischen Sprachgebrauch abweichenden Sinn: Dort versteht man unter der phonetischen Beschreibung eines oralen Wortes die Darstellung seiner lautlichen Artikulation durch eine Art von ,Partiturlesart ${ }^{14}$, gebildet aus einem Buchstaben- und Zeicheninventar, der man die Aussprache des betreffenden Wortes ablesen kann. Diese Lesart hat sich historisch stets an bestimmten oralen Dialekten orientiert, ist aber - wie bereits gezeigt in letzter Instanz immer die literale, d. h. pertinente Konstruktion der ,Aussprache' eines Wortes, das als ein Wort trivialer wie buchstäblicher Weise auch nur in dieser Konstruktion existiert. Gleiche oder ähnliche Artikulationen werden entsprechend auch gleich oder ähnlich dargestellt, ,weiß' und ,weise' etwa als /vaes/ und /vaeze/. Keinem Phonetikum eines komplexen Hanzì jedoch ist seine Aussprache abzulesen. Sein Graphismus gibt darüber keinerlei Information: Kein Fragment des Phonetikums 马 etwa lässt sich als /m/ oder als /a/ lesen. Man muss die Gesamtlesart des Zeichens kennen. Das macht die Hanzì von jedem oralen chinesischen Dialekt in einer Weise unabhängig, wie sie für eine Alphabetschrift undenkbar wäre. Die Funktion der Repräsentation des oralen Wortes, der sie ihre Konstitution verdankt, fesselt sie einerseits an diese, je länger, je mehr. Andererseits deutet schon jede ,tiefe' Orthographie wie die des Englischen - und für Sprecher schwäbischer wie niederdeutscher Dialekte dürfte die Orthographie des Schriftdeutschen ähnlich ,tief' sein - darauf hin, dass eine Schriftsprache, je, älter' sie wird, sich um so mehr von der engen Bindung an jeden oralen Dialekt emanzipiert. Das Verhältnis kehrt sich sogar um: Der ,Hochdeutsch' genannte heutige Normdialekt verdankt sich einer schriftlichen Normierung, die im 16. Jahrhundert beginnt und Ende des 19. Jahrhunderts ,kodifiziert' wird. Die Funktion der Repräsentationsfunktion wandelt sich in die der Präskription. Die Schrift emanzipiert sich und gewinnt gegenüber der oralen Sprache eine gewisse Autonomie, die jedoch eine relative bleibt, ${ }^{15}$ nicht nur wegen der

14 Vgl. Christian Stetter: System und Performanz. Symboltheoretische Grundlagen von Medientheorie und Sprachwissenschaft. Weilerswist 2005, S. 127-134. 15 Hartmut Günther und Otto Ludwig: Schrift und Schriftlichkeit. Ein interdisziplinäres Handbuch internationaler Forschung. Berlin, New York. 
präskriptiven Funktion, die die literale Sprache gegenüber der oralen gewonnen hat, sondern insbesondere wegen des morphematischen Prinzips, das in jeder Orthographie einer Alphabetschrift im Zuge ihrer Evolution mehr oder weniger stark ausgebildet wird. Einerseits stattet es den ,kompetenten' Leser, seiner' Schrift mit einer Art Autonomie gegenüber dieser aus: ${ }^{16}$ am Ende wird er jeden in seiner Schrift geschriebenen Text lesen können, auch wenn er möglicherweise nicht jedes Wort versteht. Andererseits bindet genau dies - jenseits der phonetischen oder phonematischen Repräsentationsfunktion - orale und literale Sprache auf einer strukturell höheren Ebene um so enger aneinander: eine Dialektik eigener Art.

Absolute Autonomie gegenüber der oralen Sprache gewinnt die Schrift erst in den sogenannten formalen Sprachen von Logik oder Mathematik, die ja Schriftsysteme sind. In diesen können Argumentationen und Beweise mit rein literalen Mitteln geführt werden, und dies ist auch nur insofern möglich, als jedes Zeichen eines derartigen symbolischen Systems und die mit oder über ihm möglichen Operationen wohldefiniert sind. Ein einfaches Beispiel dafür ist die Wahrheitstafelmethode der Aussagenlogik. Auch dies ist von höchster philosophischer Relevanz: Hier eröffnen sich im Medium der Schrift aufgrund seiner Pertinenz, die Definitionen von Sprachgebräuchen ebenso ermöglicht wie sie die Überprüfbarkeit des Textes garantiert, Argumentationsweisen und Erkenntnismöglichkeiten, die dem Medium der oralen Sprache aufgrund seiner Fluktuanz prinzipiell nicht gegeben sind.

1. Halbband 1994, 2. Halbband 1996; Ursula Enderle: Autonomie der geschriebenen Sprache? Zur Theorie phonographischer Beschreibungskategorien am Beispiel des Deutschen. Berlin 2005, Kap. 1 = S. 11-16.

16 Vgl. Stetter: System und Performanz (wie Anm. 4), S. 145-147. 



\section{BUCHSTABEN DER WELT}



HELWIG SCHMIDT-GLINTZER

\section{EINE SCHRIFT, DIE KEINER KENNT}

\section{Ostasiatische Schriftzeichen und das}

„Alphabet der Bitternis“ - Bemerkungen

zum Traum von einer Begriffsschrift

\section{VORBEMERKUNGEN - DIE BESONDERHEIT DES CHINESISCHEN}

Ich beginne mit einem Gedicht von Günter Eich (1907-1972), der nach abgebrochenem Ökonomie- und Sinologiestudium in den Jahren 1925 bis 1932 in Leipzig, Berlin und Paris als freier Schriftsteller lebte, dann von Berlin aus gelegentlich in den Ostseebadeort Poberow fuhr, wo folgendes Gedicht entstand:

\section{Winterliche Miniatur}

Übers Dezembergrün der Hügel

eine Pappel sich streckt wie ein Monument.

Krähen schreiben mit trägem Flügel

eine Schrift in den Himmel, die keiner kennt.

In der feuchten Luft gibt es Laute und Zeichen:

die Hochspannung klirrt wie Grillengezirp, die Pilze am Waldrand zu Gallert erbleichen, ein Drosselnest im Strauchwerk verdirbt,

der Acker liegt in geschwungenen Zeilen, das Eis auf den Pfützen zeigt blitzend den Riß, Wolken, schwanger von Schnee, verweilen überm Alphabet der Bitternis. 
Am Himmel eine Schrift, die keiner kennt - über einem Alphabet der Bitternis. - Das waren Fluchtvorstellungen mit Blick nach dem Fernen Osten.

Eine andere Perspektive nimmt Samuel Johnson im Gespräch mit James Boswell ein (Auszug aus einem Gespräch am 8. Mai 1778):

Johnson called the East-Indians barbarians.

- Boswell: "You will except the Chinese, Sir?"

- Johnson: "No, Sir."

- Boswell. "Have they not arts?"

- Johnson: "They have pottery."

- Boswell: "What do you say to the written characters of their language?"

- Johnson: "Sir, they have not an alphabet. They have not been able to form what all other nations have formed."

- Boswell: "There is more learning in their language than in any other, from the immense number of their characters."

- Johnson: "It is only more difficult from its rudeness: as there is more labor in hewing down a tree with a stone than with an axe." 1

Die chinesische Schrift hat immer schon Rätsel aufgegeben, und sie hat die verschiedensten Deutungen zur Folge gehabt, auch in China. Das hängt auch damit zusammen, dass es einerseits immer eine Art Missverständnis über den Charakter der chinesischen Schrift gegeben hat - d. h. ob es sich um eine ureigenste chinesische Entwicklung oder doch um ein Resultat der Vermischung unterschiedlicher Kultureinflüsse handelt -, und andererseits aber die Schrift einen solchen Zauber entfaltete, dem sich auf Dauer keiner entziehen konnte. Das Missverständnis soll hier nur kurz dargelegt werden, und dem Zauber will ich mich dann etwas ausführlicher widmen.

Das Missverständnis hängt damit zusammen, dass mit der Vereinheitlichung der Schrift und der Durchsetzung einer Vereinheitlichung der Verwaltung im ganzen Herrschaftsgebiet - ein Prozess, der sich wohl vor allem im Laufe des ersten vorchristlichen Jahrtausends abspielte und der natürlich zunächst ein viel kleineres Territorium umfasste als das heutige China -, dass also mit dieser Vereinheitlichung ein Schriftsystem zum

1 Zit. nach George Birkbeck Hill (Hrsg.): Boswell's Life of Johnson. Durchges. von L. F. Powell. New York 1986 (1934). 6 Bde., hier Bd. 3, S. 339. 
Standard wurde, dem sich alle Sprachen anzupassen hatten. Nicht alle haben sich daran gehalten, und einige Minoritätensprachen etwa im Südwesten waren bis ins 20. Jahrhundert dieser Akkulturation ferngeblieben und wurden dann nur alphabetisch verschriftet. Andere Regionalsprachen aber integrierten sich - und nur so ist die heutige Dialektvielfalt zu erklären, die nicht als Auseinanderentwicklung einer „Ursprache“, sondern als Folge der Integration von zunächst gänzlich unterschiedlichen Sprachen $\mathrm{zu}$ verstehen ist. ${ }^{2}$

Damit hängt auch zusammen, was gemeinhin als Diglossie beschrieben wird, nämlich die starke Differenz zwischen gesprochener und geschriebener Sprache. Diese Differenz war in China besonders groß, und es war dieser Abstand, der um 1900 dazu führte, dass grundlegende Reformen gefordert wurden, von manchen sogar die Abschaffung der Schrift und die Einführung des lateinischen Alphabets. ${ }^{3}$ Mit dieser Spreizung hängt aber auch jener Zauber zusammen, auf den ich später zurückkommen will. Zunächst aber will ich mich den Ursprüngen dieser Schrift widmen.

\section{DIE URSPRÜNGE CHINAS IN SEINER SCHRIFT}

Die Anfänge der chinesischen Schrift liegen bis heute noch weitgehend im Dunkeln. Aus den letzten Jahrhunderten des 2. vorchristlichen Jahrtausends stammende und fast unvermittelt in großer Zahl auftauchende Texte lassen den Schluss zu, dass vorher eine längere Entwicklung der Schrift stattgefunden haben muss. Insbesondere aber die früh formulierte Vorstellung, dass es sich bei der Schrift im Grunde um eine Naturerscheinung handelt, ist für die Entwicklung der chinesischen Literatur bestimmend gewesen. Der Gedanke, dass die Schrift etwas der Natur Abgeschautes sei, wird bereits in dem als Xici zhuan („Abhandlung zu den angefügten Urteilen“) oder Dazhuan („Große Abhandlung“)

2 Siehe hierzu Robert H. Gassmann, Wolfgang Behr: Antikchinesisch - Ein Lehrbuch in drei Teilen. Bd. 3: Grammatik des Antikchinesischen. Bern 2005, S. 387-407, und Wolfgang Behr: Role of Language in Early Chinese Constructions of Ethnic Identity. In: Journal of Chinese Philosophy 37 (2010), S. 567-587.

3 Siehe Elisabeth Kaske: The Politics of Language in Chinese Education, 1895-1919. Leiden 2008; zur Diglossie siehe Erhard Rosner, Schriftsprache - Studien zur Diglossie des modernen Chinesisch. Bochum 1992. 
bezeichneten Text im Yijing („Buch der Wandlungen“) formuliert und immer wieder aufgegriffen. Noch im 11. Jahrhundert schreibt der vor allem als Reformer in die Geschichte eingegangene, aber auch als Meister des Essays berühmte Wang Anshi (1021-1086) in einem zur Vorlage beim Kaiser verfassten Bericht über sein neues Werk Zishuo („Erklärung der Schriftzeichen"):

Obwohl die Schriftzeichen von einem Menschen ausgeführt wurden, haben sie ihren Ursprung doch in der Natur. Die (Fünf) Musterungen (wen) des Phönix und die Zeichnung (hua), die (auf dem Rücken eines Drachens) aus dem Gelben Fluss erschien, sind nicht von Menschen gemacht, sondern wurden von diesen nur nachgeahmt. ${ }^{4}$

Damit verweist Wang Anshi auf die himmlischen Bestallungsurkunden Hetu und Luoshu, Gelbe Fluss-Tafel und Luo-Fluss-,Schrift', von denen es im Teil Xici zhuan des Yijing heißt: „Der Gelbe Fluss brachte eine Tafel, der Luo-Fluss eine Schrift hervor. Der Heilige benutzte sie als Muster." Dieses Verständnis von der Natürlichkeit der Schrift legte den Gedanken nahe, dass es sich bei allem Geschriebenen um etwas der Weltordnung im weitesten Sinne Entsprechendes handeln müsse, und führte zu dem Schluss, dass Literatur der Ausdruck eben dieser jeweiligen Ordnung sei und darüber hinaus die Pflege der Literatur auf die Ordnung der Welt zurückwirke. Daraus erklärt sich die von den Anfängen bis in unsere Tage in China feststellbare unmittelbare Beziehung zwischen Literatur und Politik, aber auch die Bedeutung, welche man der Handschrift als Ausdruck der Persönlichkeit und ihrer Beziehung zur Weltordnung beimaß.

Heute allerdings scheint auch diese Gewissheit zerbrochen. Die mit der Herausforderung durch den Westen entstandene Sinnkrise dauert an, und die mit der Revolution in Frage gestellten Begründungsstrukturen sind weithin offen. Der Himmelssohn und der Anspruch auf Geltung für die Ordnung der Welt blieben zerbrochen. So war es nur folgerichtig, dass der Künstler Xu Bing 徐冰 (Jahrgang 1955) sein „Buch vom Himmel“ (tianshu 天書) öffentlich vorlegte, im Oktober 1988 in der China Art Gallery in Peking, in dem die Tausende von Schriftzeichen bei näherem Hinsehen alle keine Schriftzeichen mehr waren, sondern nur noch die Anmutung von Schriftzeichen hatten. Es war ein Kulturschock, eine

4 Wang Linchuan ji. Taipei 1959, Kap. 68, S. 354. 
Dekonstruktion. ${ }^{5}$ - Wenn dann im Sommer 2008 Xu Bing eine LifestyleZeitschrift mit dem Cover-Titel aufmacht: „Der Himmel beschützt China“, während im Inneren lauter Briefumschläge lagen mit Bildern von durch das Erdbeben in Sichuan verwüsteten Orten, vor allem Grundschulen mit Eltern, die den Verlust ihres einzigen Kindes beklagen, dann wird diese Parole „Der Himmel beschützt China“ zur einzigen Ironie, zur Provokation! ${ }^{6}$

Brüche zur Tradition hatte es allerdings schon seit langem gegeben, und am stärksten war wohl jener um 1900, als man China neu zu erfinden sich anschickte und dabei auch die alte Schrift abschaffen wollte. Bis heute gibt es namhafte Vertreter dieser Tendenz, die in den Spuren des Samuel Johnson wandeln.

So bewertet John DeFrancis (1911-2009) die chinesischen Schriftzeichen folgendermaßen: „Linguistically speaking, the only good thing that can be said about Chinese Characters is that they help solve problems which their own use has created."7

Doch inzwischen gibt es nach einer Infragestellung der Programmatiken der 4.-Mai-Tradition (exemplarisch: Li Xuejins 走出疑古時代) einen erneuten Paradigmenwechsel hin zur Betonung der Einzigartigkeit und Überlegenheit der Schriftzeichen gegenüber dem Alphabet; dafür steht die Apotheose der zwei in den Wüstensand (womit ja wiederum auf den Xici zhuan-Bericht zurückgegriffen wird!) geschriebenen Zeichen 天下 in Zhang Yimous Hero. (Man müsste sich einmal in den Internetforen umtun, um diesem Paradigmenwechsel nachzuspüren.)

\section{KOMMUNIKATION MIT DEN AHNEN, DEN GÖTTERN UND DER AUSSENWELT}

In China blieb die seit den Inschriften auf Orakelknochen und Schildkrötenpanzern (ca. 1250-1000 v. Chr.) und den Bronzeinschriften (ab 11. Jh. v. Chr.) etablierte Schriftlichkeit praktisch für alles Handeln bis

5 Siehe Howard Y. F. Choy: Playing with a Language of Six Thousand Years: Xu Bing's A Book from the Sky. In: minima sinica 1 (2010), S. 60-78. 6 Siehe die Abbildungen in Helwig Schmidt-Glintzer: Wohlstand, Glück und langes Leben. Chinas Götter und die Ordnung im Reich der Mitte. Frankfurt a. M. 2009, S. 389 und S. 391.

7 Siehe John DeFrancis: Foreword. In: Wm. C. Hannas: Asia's Orthographic Dilemma. Honolulu 1997, S. viii.

8 Weitere Recherchen hierzu wären wünschenswert. 
in die Gegenwart bestimmend und prägte auch den Veränderungsprozess in der von manchen als "Late Imperial China“ bezeichneten Frühen Neuzeit. Diese Dominanz der Schriftlichkeit hatte sich seit der Bronzezeit in den Kulten und der religiösen Kommunikation mit den Göttern, Geistern und Ahnen herausgebildet. Dabei standen Texte im Vordergrund, die schon in der Frühzeit ein eigenes Gewicht gewonnen hatten, selbst jene der Wahrsager. Dabei ist es ein bis heute nicht befriedigend erklärtes Phänomen, dass mit dem ersten Auftauchen von Schriftdokumenten um 1250 v. Chr. bereits ein Repertoire von mehreren tausend Schriftzeichen existierte, ${ }^{9}$ dessen Umfang sich in den folgenden Jahrhunderten vervielfachte. Dies geschah als Folge von Ausdifferenzierungsprozessen ebenso wie zur Integration und Adaptierung der kulturellen Vielfalt auf dem Territorium des heutigen China. ${ }^{10}$ Schriftlichkeit wurde so sehr als Erfolgsrezept der chinesischen Zivilisation angesehen, dass benachbarte Völker entweder die chinesische Schrift übernahmen oder doch für ihre z. T. grundlegend andersartigen Sprachen (wie z. B. das Japanische oder Koreanische) Schriften nach dem Vorbild Chinas entwickelten.

So wie sich in den im chinesischen Mittelalter entwickelten Höllenvorstellungen die Hierarchie der Bürokratie und das Personal des Kaiserhofs spiegelt, so findet auch dort Schriftlichkeit Anwendung, wenn auch in abgewandelter Form, als Geheimschrift oder in Schriftamuletten. ${ }^{11}$

Die für den Erfolg in den Palastprüfungen als der Zugangsvoraussetzung für den öffentlichen Dienst erforderlichen Kenntnisse der kanonisierten Schriften beförderte die Schriftlichkeit ebenso, wie die Verwaltung und die Durchsetzung geltenden Rechts der Schriftlichkeit bedurften. Die vielfältigen sozialen und wirtschaftlichen Veränderungen im Gefolge einer rapide wachsenden Bevölkerung (von 85 auf über 250 Mio. von 1400 bis 1650) wirkten sich auch auf das Bildungs-, Schul- und Examinierungswesen aus, ${ }^{12}$ so dass sich zu Beginn des 19. Jahrhunderts

9 Robert W. Bagley: Anyang writing and the origin of the Chinese writing system. In: Stephen D. Houston (Hrsg.): The First Writing. Script Invention as History and Process. Cambridge 2004, S. 190-249.

10 Mark Edward Lewis: Writing and Authority in Early China. Albany 1999.

11 Siehe Monika Drexler: Daoistische Schriftmagie. Interpretationen zu den Schriftamuletten Fu im Daozang. Stuttgart 1994.

12 Siehe Benjamin A. Elman: A Cultural History of Civil Examinations in Late Imperial China. Los Angeles 2000, und Lee Thomas H. C. Lee: 
dann eine sich zunehmend verschärfende Krise dieses Systems der Rekrutierung durch Bildung abzeichnete. Die Abschaffung des traditionellen Prüfungssystems 1905 war deshalb nur eine notwendige Konsequenz aus dieser Entwicklung.

Dem Bedürfnis nach Kanonisierung entsprach insbesondere nach Einführung des Buchdrucks im 9. Jh. ein Bedürfnis nach Kontrolle, ${ }^{13}$ das insbesondere in den Verbots- und „Inquisitionslisten“ der Mandschu-Zeit (zweite Hälfte 18. Jh.) einen Höhepunkt fand ${ }^{14}$ Die Publikation von großen Textkorpora in gedruckter Form, einschließlich des buddhistischen Kanons in tibetischer Sprache, erfolgte wegen dieser unhintergehbaren Bedeutung der Schriftlichkeit. Besonders unter der Mandschu-Herrschaft (seit 1644) wurden umfangreiche Editionsvorhaben sowohl von staatlicher Seite wie auch durch private Förderer durchgeführt. ${ }^{15}$ Das durch weiter entwickelte Buchdrucktechnik und die Nachfrage nach Literatur hoch entwickelte Verlagswesen ${ }^{16}$ begünstigte überlieferungskritische Editionsvorhaben, wodurch die Überlieferung ebenso wie die Geschichtsschreibung und die Wissensorganisation insbesondere seit dem 18. Jahrhundert auf eine neue Grundlage gestellt wurde. ${ }^{17}$

Die Möglichkeit der Aufzeichnung von Sprache hatte im Rahmen der Entstehung von neuen Öffentlichkeiten und einer das Land überziehenden Unterhaltungskultur zur Verbreitung von Opern- und Singspiel-Libretti geführt, in denen seit der Zeit der Mongolenherrschaft (1271-1368)

Education in Traditional China. A History [Handbook of Oriental Studies]. Leiden, Boston, Köln 2000.

13 Hok-lam Chan: Control of Publishing in China. Past and Present: The Forty-fourth George Ernest Morrison Lecture in Ethnology 1983. Canberra 1983.

14 L. C. Goodrich: The Literary Inquisition of Ch'ien-lung. Baltimore 1935; 2. Auflage New York 1966.

15 Helwig Schmidt-Glintzer: Geschichte der chinesischen Literatur von den Anfängen bis zur Gegenwart. München 1990, S. 377-482; S. 47-51; s. a. Benjamin A. Elman: From philosophy to philology. Intellectual and social aspects of change in late imperial China. Los Angeles 2001.

16 Joseph P. McDermott: A Social History of the Chinese Book. Books and Literati Culture in Late Imperial China. Aberdeen, Hong Kong 2006; sowie L. Chia: Of Three Mountains Street: The Commerical Publishers of Ming Nanjing. In: C. J. Brokaw und K. W. Chow (Hrsg.): Printing and Book Culture in Late Imperial China. Berkeley 2005, S. 107-151.

17 Kai Wing Chow: Publishing, Culture, and Power in Early Modern China. Stanford 2004. 
verstärkt Elemente der gesprochenen Sprache wiedergegeben wurden. Damit und mit der Zunahme einer städtischen Leserschaft in den Zeiten wirtschaftlicher Prosperität im 16./17. Jahrhunder ${ }^{18}$ wurde Schriftlichkeit und literarische Kultur über den engeren Kreis der Bildungselite hinaus zum bestimmenden Bestandteil der chinesischen Kultur. ${ }^{19}$

Die Schriftlichkeit Chinas führte im Westen zu Spekulationen über den Ursprung des Chinesischen, so dass etwa der vor allem als Architekt bekannte John Webb (1611-1672) im Jahre 1669 behauptete, in China habe sich die Sprache Adam und Evas erhalten. Die Bewunderung für die Literaten Chinas und die durch literarische Bildung ihrer Beamtenschaft geprägte Zivilverwaltung Chinas bestimmte weithin das Chinabild im Europa der Frühen Neuzeit. Wie sehr die Schriftlichkeit an die chinesischen Schriftzeichen gebunden blieb, zeigen auch die Bemühungen um den Druck chinesischer Schriftzeichen in Europa. ${ }^{20}$ Insbesondere die Jesuiten bemühten sich um die Aneignung nicht nur der Sprache, sondern auch der Schrift des Chinesischen.

Die erwähnte kulturelle Neubesinnung zu Beginn des 20. Jahrhunderts führte deswegen vielleicht nur scheinbar zu einem Bruch. ${ }^{21}$ Vieles spricht dafür, dass sich die Traditionen der Schriftlichkeit im China des 21. Jahrhunderts fortsetzen werden, ungeachtet auch der durch die neuen Medien verursachten Verschiebungen.

18 A. E. McLaren: Constructing New Reading Publics in Late Ming China. In: Brokaw und Chow (Hrsg.): Printing and Book Culture in Late Imperial China (wie Anm. 16), S. 152-183.

19 Schmidt-Glintzer: Geschichte der chinesischen Literatur von den Anfängen bis zur Gegenwart (wie Anm. 15), S. 400-482.

20 Georg Lehner: Der Druck chinesischer Zeichen in Europa: Entwicklungen im 19. Jahrhundert. Wiesbaden 2004.

21 Siehe auch Christoph Harbsmeiers Neuinterpretation der baihua-Bewegung als ein mit der nationalsprachlichen Formierung des Russischen, Dänischen, Norwegischen, Deutschen, Türkischen und Italienischen vergleichbares Phänomen der „Hellenisierung“, d. h. dass die literarischstilistischen, rhetorischen und linguistischen Modernisierungstendenzen als ein komplexer Prozess, die zwar durch die Begegnung mit dem Westen angestoßen, aber wesentlich auf frühere, nachbuddhistische autochthone Entwicklungen der Grammatik, Logik und Rhetorik aufbauen; vgl. Christoph Harbsmeier: „May Fourth Linguistic Orthodoxy and Rhetoric“. In: Michael Lackner, Iwo Amelung und Joachim Kurtz (Hrsg.): New Terms for New Ideas. Western Knowledge and Lexical Change in Late Imperial China. Leiden u. a. 2001, S. 373-410. 


\section{BILDER ODER ZEICHEN? - DIE ÄSTHETIK DER WELT UND DAS GESTALTHAFTE DER SCHRIFT}

Die Rolle, die die Handschrift spielte, hing von den Anfängen an aufs engste mit den Schreibtechniken zusammen. Bevor sich in China Pinsel, Tusche und Papier als Schreibutensilien einbürgerten und eine eigene ästhetische Tradition einschließlich der Grundlagen für die Malerei schufen, war eine Vielzahl weicherer oder härterer Materialien zur Beschriftung verwendet worden, Materialien tierischer Herkunft wie Knochen, Muscheln, Elfenbein und Seide, solche mineralischen Ursprungs wie Bronze, Eisen, Gold, Silber, Stein, Jade und Ton oder solche vegetabiler Herkunft wie Bambus und Holz. In der Verwendung all dieser Materialien erreichten die Chinesen ein vergleichsweise hohes Maß an Verfeinerung, jedoch wurden erstaunlicherweise niemals Schriftträger wie Tierhäute oder Pflanzenblätter verwendet.

Bereits in den Texten auf den Orakelknochen ist das Chinesische weit davon entfernt, eine reine Bilder- oder Begriffsschrift zu sein. Das Chinesische der Orakelknochen weist schon alle von späteren Kommentatoren unterschiedenen sogenannten „sechs Bildungsarten für die Schriftzeichen“ (liushu 六書) auf, woraus auf eine lange vorausgehende Schriftentwicklung geschlossen werden kann. Bei der Bestimmung der liushu sind sich die Gelehrten im einzelnen wie in der Reihenfolge zwar nicht immer einig. Im allgemeinen werden aber die Schriftzeichen entsprechend ihrer Bildung folgenden sechs Gruppen zugeordnet:

1. „Die Form abbilden“( xiangxing 象形; Piktogramme). Diese Zeichen sind Abbildungen des Gegenstandes, der durch sie benannt wird, was etwa bei den Zeichen für „Sonne“, „Mond“, „Mensch“, „Schaf“ der Fall ist.

2. „Auf eine Sache oder einen Sachverhalt verweisen“ (zhishi 指事; Symbole). Hierbei handelt es sich um Begriffszeichen wie jene für die Zahlen oder um aus Bildzeichen mit einem oder mehreren $\mathrm{Zu}-$ satzstrichen gebildete Ausdrücke wie jene für „Wurzel“ (ben 本) oder „Wipfel“ ( $m o$ 末).

3. „Vereinigung von Bedeutungen“ (huiyi 會意). Durch Kombination von zwei Begriffszeichen kommt ein Zeichen mit einer neuen Bedeutung zustande, wie im Zeichen ming (明 Sonne + Mond = klar, hell; verstehen, erklären) oder im Zeichen nan 男 (Feld + Kraft $=$ Mann).

4. „Angeglichene Lautung“ (xiesheng 諧聲). Hierbei handelt es sich um solche Zeichen, in denen ein Bestandteil des Zeichens den Laut 
angibt; entsprechend hat man gelegentlich diesen Teil „Lauter“ und den anderen Teil des Zeichens „Deuter“ genannt. In dem zhong 忠 ausgesprochenen Zeichen für „Loyalität“ etwa, das aus Zeichen zhong („Mitte, treffen") und xin („Herz") zusammengesetzt ist, ist zhong der „Lauter" und xin der „Deuter“.22 Diese Form der Zeichenauflösung zeigt bereits die Begrenztheit solcher Systematisierung.

5. „Abgeleitete Bedeutung“ (zhuanzhu 轉注). Zu dieser Zeichengruppe gehören solche bereits vorhandenen Zeichen, die für neue Wörter verwendet werden und damit eine zusätzliche Bedeutung erhalten.

6. Bei der „Entlehnung“ (jiajie 假借) handelt es sich um die stellvertretende Verwendung solcher Zeichen, die gleich ausgesprochen werden.

Während die auf den Orakelknochen belegten Schriftzeichen in der Mehrzahl der ersten der sechs Gruppen, den Bildern, zuzurechnen sind, gehört um $100 \mathrm{n}$. Chr. bereits die ganz überwiegende Zahl der geläufigen Schriftzeichen der vierten Gruppe (Zusammensetzung aus Bedeutungsund Lautbestandteil) an. Von den etwa 9500 Schriftzeichen des Shuowen jiezi („Aufklärung über die Schriftzeichen“), eines im Jahre $121 \mathrm{n}$. Chr. dem Thron vorgelegten Wörterbuches, sind dies 7697 Zeichen, während 1167 Zeichen, das sind mehr als 10 Prozent, der dritten Gruppe huiyi (Kombination von zwei Begriffszeichen) zugewiesen werden müssen. Die Anzahl der Schriftzeichen der ersten Gruppe hat demnach gegenüber der Shang-Zeit abgenommen.

Neben der Bedeutung, die der Lexikographie und überhaupt philologischen Studien, d.h. einer wissenschaftlichen Beschäftigung mit der Sprache und Schrift, seit der Han-Zeit (221 v. Chr. bis 220 n. Chr.) stets zukam, fanden doch auch weiterhin die mythischen Berichte von dem legendären Erfinder der Schrift, Cang Jie, der Historiograph des legendären Gelbkaisers, Huangdi, gewesen sein soll, mindestens ebenso weite Beachtung. Für das allgemeine Verständnis vom Charakter der Sprache war die in diesen Berichten niedergelegte Legende sogar weit prägender als alle sonstigen philologischen Theorien. Xu Shen (ca. 30 bis ca. 124 n. Chr.), der Verfasser des bereits erwähnten ältesten umfassenden erhaltenen chinesischen Wörterbuches Shuowen jiezi stellt im Nachwort zu seinem Werk einen Zusammenhang zwischen der Einführung der in

22 Siehe den Artikel von Pang Pu über die Häufigkeit von mit dem HerzRadikal geschrifteten Schriftzeichen in den Mawangdui- und GuodianManuskripten, was u.a. für zhong 中 im Zhongyong die Bedeutung von „die sich auf das Maß der Mitte beziehende Grundhaltung“ nahelegt. 
der Orakelpraxis verwendeten Trigramme und der chinesischen Schrift her und zitiert daher ausführlich aus dem zum Textkorpus des Yijing gehörigen Xici zhuan die Legende von der Einführung der Schriftzeichen:

Als in der Urzeit Bao Xi (trad. 2852 v. Chr.) die Welt beherrschte, da blickte er empor und betrachtete die Bilder am Himmel, blickte nieder und betrachtete die Vorgänge auf Erden. Er betrachtete die Muster (wen 文) auf Vögeln und Tieren und ihre Anpassung an die Orte. Unmittelbar ging er von sich selbst aus, mittelbar ging er von den Dingen aus. Dann erfand er die acht Zeichen (das sind die Trigramme) des Yijing, um die Bilder späteren Zeiten zu überliefern. Später machte der göttliche Landmann (Shen Nong) geknotete Stricke, um die Angelegenheiten der Welt zu ordnen und zu kontrollieren. So vervielfältigte sich die Anzahl der Tätigkeiten, und es wuchs die Menge der künstlich hergestellten Erzeugnisse.

Der Schreiber des Gelbkaisers, Cang Jie, der die von Vögeln und Tieren hinterlassenen Spuren betrachtete, erkannte, dass man die verschiedenen Formen unterscheiden konnte, und schuf daher Schriftzeichen, um damit die hundert Berufe zu kontrollieren und das Volk zu beaufsichtigen. Bei all dem ließ er sich von dem Hexagramm guai leiten: ,Entschlossen muss man am Hof des Königs die Sache bekanntmachen' - was bedeutet, dass die Muster (wen) die Lehre (jiao) verbreiten und am Hofe des Königs die Kultivierung (hua) zum Leuchten bringen. ,Der Edle gewährt Vergünstigungen seinen Untertanen und scheut es, bei seiner Tugend zu verweilen.'

Als Cang Jie die Schrift schuf, hielt er sich bei der Abbildung der Gestalt an die Eigenart; daher wird dies ,Muster' [vielleicht besser: ,Graphem'] (wen) genannt. Als später Schriftgestaltungen und Töne sich miteinander verbanden, nannte man dies ,Schriftzeichen' (zi). , Grapheme' bezeichnet das Grundgerüst in der Abbildung von Dingen. ,Schriftzeichen` bedeutet eine Erweiterung der Nachahmung. Auf Bambus oder Seide aufgebracht spricht man von ,Schrift' $\left(s h u\right.$, [früher oft ,Urkunden ${ }^{6}{ }^{23}$

Die hier zum Ausdruck kommende Verbindung von Geschriebenem als Muster [Graphem] (wen) mit der Verbreitung einer Lehre (jiao) und der Kultivierung (hua) schlechthin ist zu einem Grundzug der Kultur des älteren China geworden. Zugleich hat die Anschauung von der Gewinnung

23 Shuowen jiezi zhu (Ausg. von 1808, Nachdr. Taipei 1963), Kap. 15 A, 1a-2b. 
der Schriftzeichen durch Beobachtung von den Dingen der belebten und unbelebten Natur (wu) die Auffassung gefördert, dass das Geschriebene und damit die Sprache ein Abbild der Wirklichkeit sei.

Die chinesischen Zeichen verselbständigten sich sehr früh und beeinflussten andere Schriften, unter denen das prominenteste Beispiel die Xixia-Schrift ist. Auch innerhalb Chinas gab es aus Schriftzeichen gebildete oder die Schrift imitierende Amulette, von denen bereits die Rede war. Die dekorative Funktion reicht von den Teekannen bis hin zu den freilich primär funktional verstandenen astrologischen Tafeln.

Von besonderer Bedeutung wurde die Kalligraphie als Kunstform und Ausdruck der Persönlichkeit, aber auch als Repräsentant für ästhetische Differenzierung wie bei der Beschreibung der Reimregeln in der Mitte des ersten nachchristlichen Jahrtausends oder in Form von Spielereien wie im Falle der Darstellung des Gottes der Literatur. Zudem darf man davon ausgehen, dass die Gedächtniskultur in China und speziell mnemotechnische Verfahren nicht unwesentlich durch die Zeichenkultur geprägt worden sind. Jedenfalls kam der graphischen Seite der Schrift eine sehr viel größere Bedeutung zu als in allen Alphabetkulturen. Das Problem der Legasthenie stellt sich nicht so wie in BuchstabenschriftKulturen.

Die ornamentale Verwendung der Schrift führte auch dazu, dass mit den Schriftzeichen eigene Formen gebildet und Figuren dargestellt wurden. So gibt es in China wie in anderen Kulturen Figuren-Gedichte, ${ }^{24}$ aber weit darüber hinaus sind Zeichen immer wieder zu Elementen geistiger Landschaften oder Tafeln $(t u)$ geworden.

Nachdem wir uns lange vorwiegend mit gedruckten Materialien beschäftigt haben, sind wir inzwischen auch vertrauter mit der Manuskriptkultur, das heißt mit der Schreibpraxis im älteren China, auch wenn hierzu noch viel Forschungsarbeit ansteht, womit man sich in einem Hamburger Projekt seit einigen Jahren intensiver beschäftigt. Zugleich ist das Lesenlernen ganz anders als bei Buchstabenschriften. Alle Hinweise deuten darauf, dass durch Wiederholung, durch lautes Lesen, Aussprache und Inhalt gelernt wurden - und so ein Verständnis von schriftsprachlicher Überlieferung entstand, welches eine ganze andere Referentialität zu vorgängigen Sprach- und Kulturmustern etablierte.

24 Siehe Herbert Franke: Chinese Patterned Texts. In: Dick Higgins: Pattern Poetry. Guide to an Unknown Literature. Albany 1987, S. 210-219. 


\section{SCHRIFT, EMOTIONALITÄT UND AUSDRUCK DES HERZENS}

Im Verabschiedungstext für den Mönch Gaoxian des Han Yu (768-824), einem seiner späten Texte, betont er die Einheit von Spiritualität und Literatur. ${ }^{25}$

Han Yu nämlich hatte gegenüber einem Mönch namens Gaoxian bezweifelt, dass dieser überhaupt in der Lage sei, ein guter Kalligraph zu sein, weil eben zur Kalligraphie, die überhaupt einen Anspruch erheben kann, als solche bezeichnet zu werden, genau so wie zur Lyrik kraftvolle Emotionen die Voraussetzung seien. Der Mönch, der seine Emotionen überwindet und zurücklässt, ist demnach zum künstlerischen Ausdruck nicht mehr fähig. Und gewissermaßen als Echo auf diese von Han Yu in einem Text anlässlich eines Abschieds von Gaoxian formulierten Ansicht schreibt Su Dongpo zum Abschied des ihm freundschaftlich verbundenen Canliao: ${ }^{26}$

Tuizhi [i. e. Han Yu] meinte von der Kursivschrift Sie könne sämtliche Lagen auskosten

Alle Sorgen und Aufwallungen

Könnten im Strich eines Tuschepinsels ihren Ort finden.

Doch bei einem Buddhisten,

Der sich selbst als einen leeren Brunnen sieht

Und sich selbst nur der Stille und Einfachheit (danpo 淡泊) aussetzt $^{27}$

Wer würde von ihm Kühnheit oder Gewalt erwarten?

Doch denke ich darüber nach, verhält es sich nicht so.

Wahre Fertigkeit hat nichts mit Illusion zu tun.

Will man, dass ein Gedicht wunderbar wird, Musst Du weder Leere noch Ruhe meiden.

In der Ruhe erfasst Du das Insgesamt aller Bewegungen!

In der Leere schaffst Du Raum für die Zehntausend Erscheinungen

25 Die folgenden Überlegungen sind meinen in Marbach am Neckar am 21. Januar 2011 vorgetragenen Ausführungen zur Heiterkeit in China entnommen.

26 Ronald C. Egan: Word, Image, and Deed in the Life of Su Shi. Cambridge, Mass. 1994, S. $198 \mathrm{f}$.

27 Danpo zhuyi 淡泊主义 ist übrigens eine Übersetzung für ,Stoizismus! 
Beobachtend die Welt beim Gehen unter den Menschen

Und sein Selbst betrachtend beim Sitzen auf dem umwölkten Grad.

Die Menge mag das Salzige oder das Sauere;

Doch der wahre und ewige Geschmack liegt dazwischen (in der Mitte)

Dichtung und Buddhismus schließen einander nicht aus

Das lege ich vor als Gegenposition.

Auch wenn sich Su Dongpo selbst nicht immer an „Stille und Leere“ hält, so geht er doch über die stoische Haltung der Vermeidung anklagender Töne hinaus, wie sie auch von anderen, wie etwa Ouyang Xiu, vertreten wurde..$^{28}$ Die Grundhaltung war aber eine Art von „In-der-Welt-“, nicht aber „Von-der-Welt-Sein“. Über die Fähigkeit Canliaos, Empfindungen zu haben, sagt er: „Sein Körper war verdorrt und sein Geist verbrannt, und doch liebte er Verse zu verfassen, die von den Umständen angeregt wurden, und er freute sich an den Dingen und übersah nicht die menschlichen Gefühle." Freilich hielt er sie an der Oberfläche, wie Su, wie wir oben sahen, preisend formuliert.

Um diesen Zusammenhang noch etwas weiter auszuleuchten, gehe ich nun zurück auf den Text Han Yus, der von einem selbstgenügsamen Intellekt ausgeht, der nur auf die Welt reagiert und sich nicht wirklich selbst auf die Welt einlässt:

Wenn jemand seine Fähigkeiten und sein Wissen in einem Gebiet festlegen kann, so dass er mit seinem Sinn ganz auf die Gegebenheiten eingehen kann, ohne dass seine Vitalität gebrochen wird, dann wird sein Geist ganz bleiben und er wird ungerührt sein. Die äußeren Dinge lässt er an sich heran, doch sie werden seinem Sinn (Herzen) nichts anhaben.

So haben Yao, Shun, Yu und Tang das Reich in Ordnung gehalten, so hat Yang Shu den Bogen gemeistert und der Koch Ding den Ochsen zerlegt. Meister Kuang beherrschte die Töne, Bian Que die Medizin, Liao das Jonglieren, Qiu das Schachspiel und Bo-lun (d.i. Liu Ling) den Weinkonsum. Sie alle hatten ihre Freude daran bis zum Ende ihrer Tage, ohne je zu versagen, aber auch, ohne es sich zu erlauben, sich für etwas anderes zu interessieren. Denn wer sich begeistern lässt und andere als die ihm zukommenden Tätigkeiten verfolgt, wird nicht mal in die Halle aufsteigen (Ly XI.15) oder die feinsten Happen erlangen.

28 Egan: Word, Image, and Deed in the Life of Su Shi (wie Anm. 26), S. 199. 
In früherer Zeit war Zhang Xu ein Meister in der Grasschrift (caoshu) und sonst verstand er sich auf nichts anderes. Immer wenn sein Sinn von Freude, Ärger, Mutlosigkeit, Kummer, Beglückung, Hass oder Zuneigung bewegt war, von Nüchternheit oder Trunkenheit oder Ungerechtigkeit, so brachte er dies immer in seiner Kalligraphie (der Grasschrift) zum Ausdruck. Welche Dinge er auch immer erblickte, Berge, Flüsse, Gipfel, Täler, Vögel, wilde Tiere, Insekten, Fische, Blumen, Früchte, Sonne, Mond, Sterne, Wind, Regen, Überschwemmungen, Feuersbrünste, Donner, Blitz, Gesang, Tanz, Krieg oder Waffenstillstand, alle Wandlungen des Himmels und der Erde, seien sie freudig oder betrüblich fanden alle ihren Ort in seiner Schrift. So war die Wandlungsfähigkeit seiner Kalligraphie wie die von Seelen und Geistern und blieb daher rätselhaft. Dies praktizierte er bis an sein Lebensende und blieb darob berühmt bei der Nachwelt.

Wenn nun Gao Xian die Grasschrift praktiziert, hat er da auch den Sinn des Zhang Xu? Wenn er nicht dessen Sinn hat und nur dessen Spuren folgt, so wird er niemals an Gao Xian heranreichen. Um an Zhang Xu heranzureichen, muss man jeden Nutzen und Schaden kennen, und man darf selbst die kleinsten Nuancen nicht unberücksichtigt lassen. Und wenn eine Leidenschaft entflammt, wird sie ihr Ziel suchen, und ob nun erfolgreich oder nicht, wird man daran festhalten und alles in die Schrift einfliessen lassen und so an Zhang Xu heranreichen.

Doch dieser Gao Xian nun ist ein Buddhist, hält Tod und Leben für gleichgültig und hat sich von allen äußeren Anhaftungen gelöst. Und da er seinen Sinn stets in Ruhe hält, kann dort nichts aufwallen, und gegenüber der Welt bleibt er interesselos und wird daher nichts begehren. Wenn Ruhe und Interesselosigkeit zusammen kommen, dann liegt man danieder, erschöpft, verloren und ohne Hoffnung. Wie sollte man da in der Kalligraphie nicht ganz genau so sein?

Nun habe ich gehört, dass Buddhisten gut in Illusionen (善幺𠃌) sind und darin viele Talente haben. Vielleicht ist Gao Xian darin beschlagen, doch kann ich darüber nichts wissen. ${ }^{29}$

Gegen diese Polemik also richtet sich Su Dongpo und wendet sich damit einem Weltverständnis zu, welches die Heiterkeit an die Stelle der Ergriffenheit und des inneren Feuers setzt. Es dies eine Haltung, die aus

29 Siehe Han Changli wen ji jiaozhu (Zhongguo wenxue mingzhu, 3. Serie). Taipei 1967, 157-159; vgl. Charles Hartman: Han Yü and the T'ang Search for Unity. Princeton, N. J. 1986, S. 222 f. 
der Stille heraus entsteht und doch die Abgründe nicht übersieht. Dann erst wird man offen für die Begeisterung der anderen und muss selbst die Abgründe nicht meiden. Die Weltoffenheit haben beide, der auf seine Fertigkeiten, gewissermaßen professionalisierte Experte bei Han Yu ebenso wie der „Dilettant“ bei Su Dongpo, der sich auf alles einzulassen bereit ist. Während aber Han Yu auf der Beurteilung nach Nutzen und Nachteil, nach Vorteil und Verlust beharrt, kennt der Protagonist bei Su Dongpo das interesselose Wohlgefallen.

\section{SCHLUSSBEMERKUNG}

Die Schrift war zum zentralen Selbstverständigungsmittel geworden. Noch Liang Qichao betonte in einem Vortrag über Kalligraphie im Jahre 1925: „In der Kunst gibt es auf der ganzen Welt die drei Sparten Malerei, Bildhauerei und Architektur. Nur in China gibt es eine weitere Kunstform, das Schreiben von Schriftzeichen." In der Schrift sah man Kraft (shi 势), Anmut (yun 韵) und Geist [vielleicht hier eher: Virtuosität] (shencai 神采), und in den verschiedenen Schreibstilen, die zum Teil, wie die Kleine und die Große Sigelschrift, historisch verortet wurden, sah man den Ausdruck von Bewegung ebenso wie von Gefühlen (qinggan 情感) von Freude ( $x i$ 喜), Zorn (nu 怒), Kummer ( $a i$ 哀) und Glück ( $l e$ 乐). Ja selbst Verrücktheit oder die Grenze zur Ekstase konnte sich in der Schrift ausdrücken.$^{30}$ Es gab Meister der Schreiblunst und darunter solche, die speziell einen Stil in besonderer Weise beherrschten.

Dabei trat etwas weiteres hinzu: die Zahl der Schriftzeichen vermehrte sich ständig, von den 9.353 Zeichen des Shuowen jiezi der Späteren HanZeit über die 47.035 des Kangxi cidian bis hin zu den heute weit über 53.525 Schriftzeichen. Und es werden immer mehr, einmal ganz abgesehen davon dass auch die Bedeutungsnuancen zunehmen, nicht zuletzt durch mutwillige semantische Überlagerungen und Vertauschungen infolge der staatlichen Zensur.

Diese Fülle, der Zauber der Schriftsprache und ihrer Auslegungsund Verständigungspotentiale und nicht zuletzt die mit der Schrift verknüpften Ausdrucksmöglichkeiten, die manuelle, geistige und emotionale Ebenen verknüpfen und verschränken, werden mit dazu beitragen, dass

30 Siehe Adele Schlombs: Huai-su and the Beginnings of Wild Cursive Script in Chinese Calligraphy. Stuttgart 1998. 
die Schrift des Chinesischen eine tragende Rolle in Ostasien behalten wird. Noch heute wird nicht nur in China, sondern auch in Japan, die Handschrift einer bedeutenden Persönlichkeit verehrt, und die Eingangstore zu den wichtigsten Institutionen sind geschmückt mit Schrifttafeln, die nicht von einem Designbüro stammen, sondern die Handschrift einer Persönlichkeit darstellen. - Vor diesem Hintergrund bekommt der Diskurs über Individualität und Persönlichkeit im Diskurs zwischen Ost und West eine neue Dimension. Zugleich erhält die Erforschung der Schriftlichkeit und der "Orthographie“ mit den neuen Funden der Archäologen eine neue Aufmerksamkeit. Die weitere Erforschung der Lehnschreibungen und „Fehlschreibungen“ offenbart jenseits der Alternative Lautschrift oder Schrift mit chinesischen Schriftzeichen die Komplexität der Verständigung im älteren China und weist möglicherweise den Weg zu einem neuen Umgang mit der über lange Zeit nur unter pragmatischen Gesichtspunkten bewerteten Schrift Chinas. ${ }^{31}$

31 Siehe den Rezensionsartikel von Matthias L. Richter: The Fickle Brush: Chinese Orthography in the Age of Manuscripts: A Review of Imre Galambos's Orthography of Early Chinese Writing: Evidence from Newly Excavated Manuscripts. In: Early China 31 (2007), S. 171-192. 

RYOZO MAEDA

\title{
SYMBOLISCH-IMAGINÄR?
}

\section{Manga und die ,kulturfigurative' Wirkung der japanischen Schrift}

\author{
Zum Gedächtnis meiner Mutter, \\ die auch Kalligrafie-Meisterin war
}

I.

Manga ist längst ein internationales Kulturphänomen. ${ }^{1}$ Im Kontrast zu Anime (japanischer Zeichentrickfilm), der schon seit den frühen 1980er Jahren auch in Europa in Serie ausgestrahlt worden war, erfuhr der Comic-Strips aus Japan zunächst nur zögernd eine breite Akzeptanz zumal im deutschsprachigen Raum. Es gehört indes zur visuellen Grunderfahrung von Jugendlichen im Bereich der Populärkultur. Jetzt wird Manga nicht nur übersetzt und gelesen, sondern auch von jungen europäischen Zeichnern produziert und publiziert.

Mit der Internationalisierung des Manga stellt sich die Frage: Inwiefern ist er ,japanisch'? Als visuelles Medium der Populärkultur, das sich im 20. Jahrhundert entwickelt hat, ist Manga genauso international und transkulturell geprägt wie der Film. Seine medialen Grundelemente wie

\footnotetext{
1 Zur Schreibweise der japanischen Wörter: Japanische Familiennamen werden gänzlich in Großbuchstaben wiedergegeben, wenn sie im Haupttext und in den Fußnoten zum ersten Mal erscheinen. Die Familiennamen werden den Vornamen nachgestellt, wenngleich in Japan der Familienname immer vor den Vornamen gesetzt wird. Das Dehnungszeichen über den Vokalen, die gedehnt ausgesprochen werden [ā, ō, ù, usw.] wird bei den Personen- und Ortsnamen nicht verwendet.
} 
Panel, Sprechblase und Speedline gehen direkt auf die amerikanischen Comic-Strips des frühen 20. Jahrhunderts zurück. Diese Elemente, die teilweise noch ältere westliche Ursprünge haben, werden seit den späten 1950er Jahren von japanischen Manga-Zeichnern in einer ausgesprochen originellen Weise weiter entwickelt. ${ }^{2}$ Anders als oft behauptet, handelt es sich hier also nicht um ein Medium, dessen Spezifikum primär auf traditionelle visuelle Medien Japans wie etwa Bildrolle (Emaki) zurückzuführen ist.

Dennoch ist es auch nicht zu leugnen, dass sich Manga wegen ihrer unverwechselbaren visuellen Eigenschaften von den übrigen Arten von Comics (Cartoon, Bande dessinée usw.) klar unterscheiden lässt. Nicht selten versucht man, diese Eigenschaften mit Hilfe der traditionellen japanischen Ästhetik zu erklären - nämlich als Ausdruck einer Ästhetik, die sich in ihrer kühnen Schlichtheit auszeichne. Eine solche Ästhetik, die auf den Prinzipien der Auslassung basiert, könne man nicht nur in der traditionellen Malerei, sondern auch im Steingarten eines zen-buddhistischen Tempels oder im Raum für die Tee-Zeremonie wiederfinden. Und in der Diskussion über Manga wird sie mit dessen einfacher, aber höchst ästhetisierter Visualität in Verbindung gebracht. Besteht doch unter Kennern und Fans ein Konsens darüber, dass Manga in dieser Hinsicht innerhalb des Comics der Gegenwart, der im Kontrast zu Fotografie, Film oder Malerei ohnehin als „kühles, detailarmes Medium“ („cool medium in low definition") definiert wird, ${ }^{3}$ auch eine besondere Stellung einnimmt.

Dieses Erklärungsmuster ist nicht nur in Japan bekannt und beliebt, geht allerdings mit der Gefahr einer kulturessentialistischen und eigentlich tautologischen Behauptung einher: Manga sei japanisch, denn ihm liege die japanische Ästhetik zugrunde. Dagegen wird im Folgenden versucht, seine spezifische Visualität im Zusammenhang mit der

2 Bekanntlich ist es der amerikanische Comic-Zeichner und Zeichentrickfilm-Regisseur Winsor McCay (1871-1934), der mit seiner 1905-1911 in der Tageszeitung New York Herald publizierten Comic-Serie Little Nemo in Slumberland eine freie Aufteilung der Seiten durch die Panels einführte. Davor waren die Panels des Comic-Strips wie die Einzelbilder des Films gleichförmig und von derselben Größe. Mit McCay beginnt in diesem Sinne der moderne Comic-Strip. Vgl. Inuhiko Yomota: Manga Genron (Manga Principia). Tokyo 1999, S. 32.

3 Marshall McLuhan: Understanding Media. The Extensions of Man (1964). With a new introduction by Lewis H. Lapham. Cambridge (Mass.), London 1994, S. 22. 
japanischen Schrift zu erörtern, und zwar unter besonderer Berücksichtigung des Modus der Bildwahrnehmung im Kontext der japanischen Schriftpraxis. In Frage kommt Manga dabei m. E. einerseits als visuelles Medium, in dem eine ,implosive' Konfiguration von Bild und Zeichen bis aufs Äußerste getrieben wird. ${ }^{4}$ Andererseits hängt diese konfigurierende Operation damit eng zusammen, dass Manga wesentlich dazu bestimmt sind, massenhaft und extrem schnell rezipiert zu werden.

Wie Zahlen und Daten aus den Jahren des Manga-Booms zeigen, handelt es sich um ein Paradebeispiel dafür, dass Quantität in Qualität umschlägt. Nach statistischen Erhebungen sind fast 38 Prozent der 1997 in Japan publizierten Titel insgesamt Manga-Bücher und ca. 22 Prozent der Zeitschriften auf Manga spezialisiert. 1995 betrug die Gesamtauflage der Manga-Publikation (Bücher und Zeitschriften) etwa 2300 Millionen. In der Regel wird ein Story-Manga zunächst in einem Wochenmagazin publiziert, bevor er in einer meist mehrbändigen Buchausgabe erscheint. Jump, das in den 1980er und den frühen 1990er Jahren das meistverkaufte Manga-Magazin war, erreichte 1995 einen Rekordumsatz von durchschnittlich 6,53 Millionen Exemplaren pro Woche. ${ }^{5}$

Dieser konsumierenden Massenrezeption entspricht die hohe Geschwindigkeit, mit der Manga ,gelesen' werden. Frederik L. Schodt berichtet über einen Japaner, der in nur 20 Minuten ein 320 Seiten starkes Manga-Wochenmagazin bis zum Ende durchlas. ${ }^{6}$ Ein Tempo von 3,75 Sekunde pro Seite ist allerdings für einen erfahrenen Manga-Konsumenten keine besondere Leistung. Was diesem Japaner ein solches, an westlichem Standard gemessen ,extrem' schnelles Lesen ermöglicht, sucht Schodt in einer Verwandtschaft zwischen dem japanischen Schrifttext und dem Manga im Sinne von konfigurierten Bildzeichen. Er zitiert nämlich die Aussage mehrerer Japaner, die zwischen dem Lesen eines japanischen

4 Vgl. Ryozo Maeda: Rasender/Lesender Stillstand. Zur Wahrnehmung von Schrift- und Textbild im Manga. Ryozo Maeda, Teruaki Takahashi, Wilhelm Voßkamp (Hrsg.): Schriftlichkeit und Bildlichkeit. Visuelle Kulturen in Europa und Japan. München 2007, S. 197-218, hier S. 198 (ursprünglich in: Matthias Bickenbach und Axel Fliethmann [Hrsg.]: Korrespondenzen. Visuelle Kulturen zwischen Früher Neuzeit und Gegenwart. Köln 2002, S. 89-107).

5 Sharon Kinsella: Adult Manga. Culture and Power in Contemporary Japanese Society. Honolulu 2000, S. 41-43.

6 Frederik L. Schodt: Dreamland Japan. Writings on Modern Manga. Berkeley ${ }^{2}$ 1998. S. 26. 
Texts und dem eines Manga keine prinzipiellen Unterschiede fänden. Er kommt somit zur Ansicht, dass es sich in den beiden Medien jeweils um „a streamliened visual representation of reality" handelt. ${ }^{7}$ Manga ist ihm zufolge genauso wie die japanische Sprache eine ,Bildsprache'. Hier sind Lesen und Sehen wohl keine zwei verschiedenen Tätigkeiten.

Einerseits wird die Ansicht dieses amerikanischen Manga-Spezialisten, der im seinem Erklärungsversuch kein Einzelgänger ist, auch von nicht wenigen japanischen Manga- und Anime-Zeichnern geteilt. ${ }^{8}$ Andererseits ist seine These, die die Verwandtschaft zwischen Manga und dem Japanischen in ihrem bildsprachlichen Charakteristikum sehen will, einer weiteren Differenzierung bedürftig. Eine allzu grobe Auffassung der japanischen Sprache könnte zu einer vom heutigen Stand der Sprachwissenschaft gesehen problematischen Schlussfolgerung führen wie etwa: die japanische Sprache - genauer: die japanische Schrift (écriture) - habe eine viel direktere Beziehung zum Imaginären als die alphabetische. So geht z. B. Jaques Lacan offensichtlich von der Unterstellung dieser besonderen Affinität aus, wenn er im Vorwort zur japanischen Ausgabe seiner Ecrits $I$ (1972) die Unmöglichkeit erwähnt, einen Japaner psychoanalytisch zu behandeln. Lässt sich doch diese Aussage umformulieren im Sinne: In der japanischen Schrift sei es nicht möglich, klare Trennlinien zwischen dem Symbolischen und dem Imaginären zu ziehen. ${ }^{9}$

In der japanischen Manga-Diskussion wird die folgende Auffassung ebenfalls breit geteilt: Die chinesische Schriftzeichen seien Hieroglyphen. Diese in der europäischen Denktradition tief verwurzelte Auffassung geht wohl bis auf Leibniz zurück, ${ }^{10}$ stiftet jedoch gewisse ,Verwirrungen', wenn

\section{Ebd.}

8 Isao Takahata (geb. 1935), einer der führenden Anime-Regisseure in Japan, vertritt auch diese Ansicht. Vgl. Isao Takahata: Jūniseiki no Animation. Emakimono ni miru Eigateki Animeteki narumono (Zeichentrickfilm im 12. Jahrhundert. Filmische und anime-hafte Elemente in Bildrolle). Tokyo 1999.

9 Eine solche Ansicht wird auch in der Diagnose der postmodernen japanischen Gesellschaft geteilt. Vgl. Hiroki Azuma: Dōbutsuka suru Postmodern. Otaku kara mita Nihonshakai (Postmoderne auf dem Weg zum Animalischen. Japanische Gesellschaft aus der Perspektive des Otaku). Tokyo 2001.

$10 \mathrm{Vgl}$. Brief von Leibniz an Joachim Bouvet (1703), zit. in: Jacques Derrida: Grammatologie. Übersetzt von Hans-Jörg Rheinberger und Hanns Zischler. Frankfurt a. M. 1974, S. 141. Leibniz' Vorstellung der chinesischen Schriftzeichen als Hieroglyphen steht unter dem Einfluss des ziemlich 
sie direkt auf die Wesensbestimmung der ostasiatischen Schriftkultur angewendet wird. Auf diesen Punkt komme ich am Ende der vorliegenden Ausführungen zurück.

Im Folgenden geht es in erster Linie darum, die neuere Diskussion über Wirkungen der japanischen Schrift auf Manga unter zwei Aspekten zu skizzieren: Ikonografismus und Kontextabhängigkeit. Sie beziehen sich beide in erster Linie auf den Rezeptionsmodus des Manga und der japanischen Schrift. Statt auf die oft irreführende Frage einzugehen, inwieweit die japanische Sprache eine Bildsprache sei, sollen die Betrachtungen auf die Frage konzentriert werden: In welcher Verwandtschaft können die beiden visuellen ,Medien' adäquat zusammen diskutiert werden. Mit einer solchen Beschränkung soll versucht werden, einige der Zentralaspekte der neueren Ansätze, die Genese und Formation dieses populärkulturellen Phänomens von der kulturspezifischen Schriftpraxis her zu beleuchten, umso schärfer zu konturieren.

II.

Fassen wir zunächst einmal den Zeichenbestand des Japanischen zusammen, wie er in der heutigen Schriftpraxis verwendet wird: Hauptbedeutungsträger sind einige tausend chinesische Schriftzeichen, Kanji (漢字). Und es gibt zwei phonetisch identische, aber grafisch verschiedene, je 46 Zeichen umfassende Silbenschriften, Hiragana (ひらがな/平仮名) und Katakana (カタカナ/片仮名). Hiragana werden für die Flektionsendungen der Verben, für grammatische Elemente wie Kopula und Objekt- und Themamarker sowie für Begriffswörter, deren Kanji außer Gebrauch gekommen sind, verwendet. Katakana dienen dagegen der Umschrift von westlichen Fremdwörtern, die sie deutlich als fremd aus dem Schriftbild hervortreten lassen, für Hervorhebungen sowie zur Niederschrift von Telegrammtexten. Dazu kommen chinesische und arabische Zahlzeichen, sowie Rōmaji, das lateinische Alphabet (für Akronyme, z. B. ,NHK' oder ,IBM $)$. Schließlich gibt es seit etwa hundert Jahren rein textuelle Operatoren wie Interpunktions- und Anführungszeichen. Die japanische Schrift der Gegenwart ist also ein Mischsystem aus chinesischen Schriftzeichen

verworrenen Konzepts von Athanasius Kircher, der die Ursprünge des chinesischen Schriftzeichen in den ägyptischen Hieroglyphen sucht. Vgl. Umberto Eco: Die Suche nach der vollkommenen Sprache. Übersetzt von Burkhart Kroeber. München 2002, Kapitel 7 u. 14. 
Kanji und den beiden aus ihnen entstandenen phonetischen Zeichen, Kana, sowie Rōmaji. Diese Mischform wird im Japanischen als Kanji kana majiri bun (Mischschrift von Kanji und Kana) bezeichnet und ist in ihrer Form wahrscheinlich einzigartig in der Welt.

Von ihren Anfängen im 5. Jahrhundert her ist die Schriftpraxis des Japanischen durch eine kulturelle Hybridität geprägt. Und seit der $\mathrm{He}-$ rausbildung vom phonetischen Schriftzeichen Kana im 9. Jahrhundert funktioniert die japanische Schrift auch als Medium für die Visualisierung dieser Hybridität, wie sie die Kultur Japans im Allgemeinen charakterisiert. Sichtbar gemacht wird sie gerade in der oben genannten grafischen Kanji-Kana-Mischform. Die japanische Schrift muss also als mehrfaches Palimpsest gelesen werden, in das mehrere Schichten der kulturellen Hybride eingeschrieben sind. Auf den ältesten und älteren Schichten dieses Palimpsests ist die chino-japanische Hybride aufgeschrieben, die inzwischen zur zweiten Natur in dieser Schriftkultur geworden ist. Sie ist quasi der ,Grund' dieses Schriftbildes, auf dem sich solche ,fremden' Elemente, deren Herkunft jetzt außerhalb des traditionellen chino-japanischen Kulturraums zu suchen sind, wie ein ,Muster hervorheben.

III.

Richtet man sein Augenmerk auf die besondere Zeichenhaftigkeit der Manga-Figur, so kommt die ikonografische Tendenz als eines der Hauptmerkmale in Frage, das nach einer breit geteilten Ansicht Manga mit der japanischen Schrift verbindet. ${ }^{11}$ Der Manga-Zeichner und -kritiker Fusanosuke Natsume (geb. 1950) ist einer der ersten, die den Ikonografismus zum Thema der Manga-Analyse gemacht haben. In seinem preisgekrönten Buch Manga no Chikara (Energie des Manga) aus dem Jahr 1999 weist er darauf hin, dass im Manga, dessen eigenartig schlichte Bildlichkeit bereits eingangs erwähnt wurde, sehr detaillierte Bilder mit künstlerischer Perfektion vom Lesern nicht selten als störend empfunden werden, weil damit ein schnelles Lesen verhindert wird.12

11 Hier wird leicht revidiert wiedergeben, was ich in meinem Aufsatz: Rasender / Lesender Stillstand (wie Anm.4) bereits veröffentlicht habe.

12 Fusanosuke Natsume: Manga no Chikara. Seijuku suru Sengo Manga (Energie des Manga. Zur künstlerischen Reifung des Nachkriegsmanga). Tokyo 1999, S. 14-21. 
Grundkonzeption der ,vereinfachenden' Operation sucht Natsume im Buch Manga no Kakikata (Wie Manga zeichnen) von Osamu Tezuka (1928-1989), das 1956 erschien und heute als Klassiker der MangaTheorie gilt. Tezuka, der ,Vater' des Gegenwartsmanga und -Anime, präsentiert hier systematisch eine neue Methode, wie man einen psychischen Vorgang einer Person in einer schlichten Darstellung , sichtbar ${ }^{6}$ macht. Er hat somit einen wesentlichen Teil der,Grammatik des Manga' erfolgreich entworfen.

Das ikonografische Verfahren, das Tezuka demonstriert, liegt erstens darin, das Gesicht einer Person in seine ,Grundelemente' wie die Augen, die Nase, den Mund und die Augenbrauen zu zerlegen, die dann zu einem bestimmten Mienenausdruck wieder zusammengestellt werden. Was Tezuka hier entwickelt, könnte man als physiognomisches System der Kombination bezeichnen. ${ }^{13}$

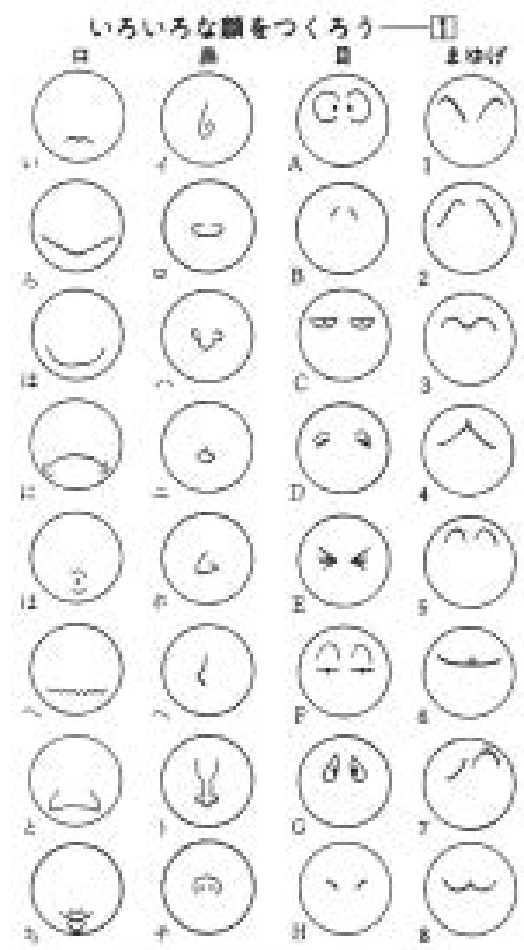

1 Grundelemente des Mienenausdrucks

13 Hierzu vgl. auch: Yomota: Manga Genron (wie Anm. 2), S. 142-150. 


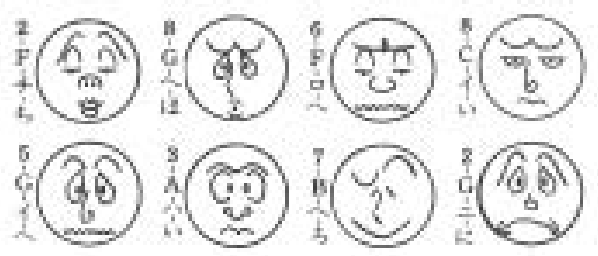

2 Mienenausdrucke als zusammengestellte Grundelemente

Zweitens ordnet Tezuka eine ganze Reihe der zusätzlichen Hilfszeichen $(M a n p u)$ in ein umfassendes semantisches System ein. Dieser Zeichen bedient man sich, um einen bestimmten psychologischen Vorgang bzw. eine Körperbewegung noch deutlicher zum Ausdruck zu verhelfen. In diesem System stehen z. B. ,triefende Schweißtropfen', die einer Menschenfigur zugefügt werden, für Angst, Schock oder Unsicherheit. Ein schwarzes Wölkchen über dem Kopf stellt dagegen Unzufriedenheit oder Frustration dar. Solche Zeichen gibt es zwar auch im Comic allgemein, sogar bereits in europäischen Karikaturenbildern des 19. Jahrhunderts. Aber keiner hatte vor Tezuka unternommen, sie in ein so umfassendes semantisches System einzuordnen.

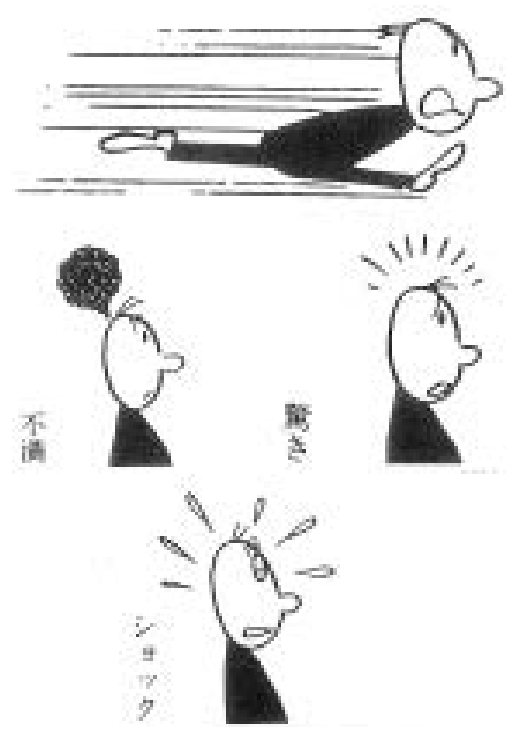

3 Zusätzliche Hilfszeichen 
So sieht Natsume den großen Verdienst Tezukas darin, mit seiner ,Physiognomik' möglichst verschiedene Muster des Mienenausdrucks entwickelt und somit die Ausdrucksmöglichkeit des Manga sprunghaft erweitert zu haben. Wie oben skizziert, besteht diese ,Physiognomik' aus einem Doppelverfahren: Zergliederung in die semantischen Grundelemente und Kombination dieser Elemente zu einer Figur. Der gleiche ikonografische Vorgang gilt auch der Ergänzung der Figur mit einem Manpu. Inzwischen gehört ein solcher Ikonografismus zu einer Grundoperation auch der Gattung Gekiga (Bilddrama), die sich im Kontrast zu den Comics für Kinder durch ihre detailliertere Zeichnungsweise charakterisiert.
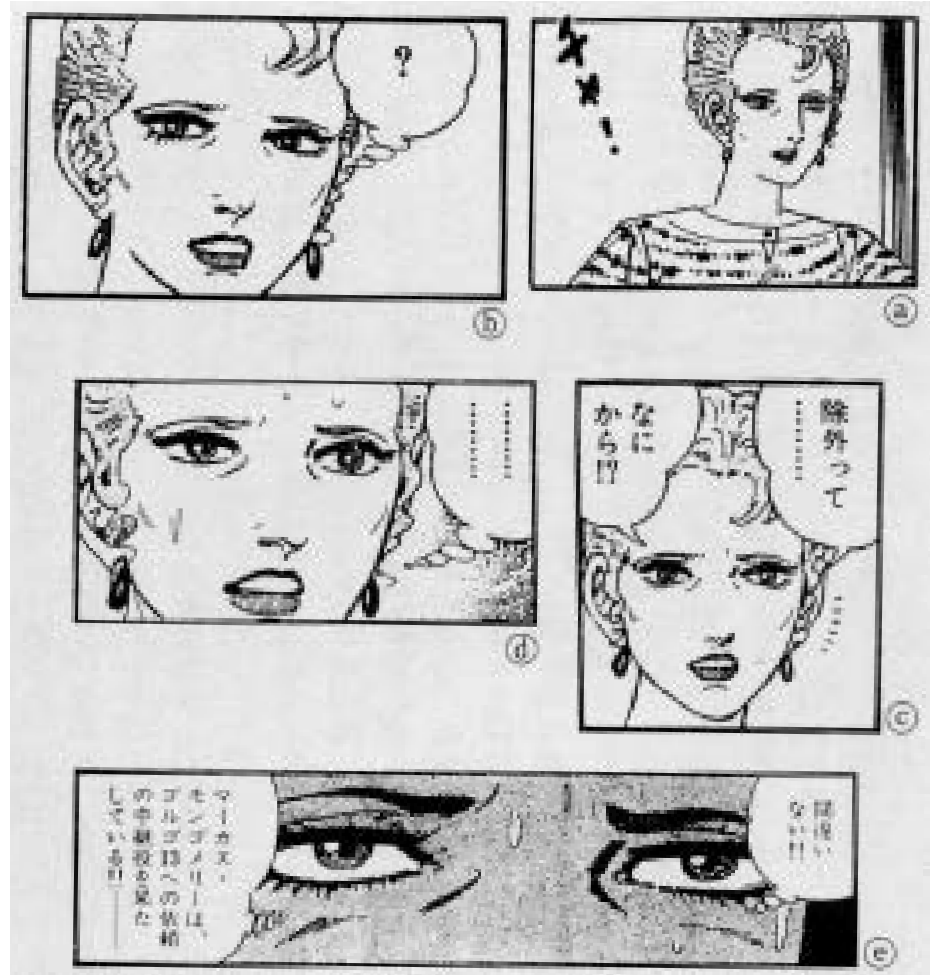

4 Hilfszeichen in Gekiga

Diese Zeichenhaftigkeit der Manga-Figuren versucht Natsume in ihrer analogischen Beziehung zum mit chinesischen Schriftzeichen zu erklären. Geprägt wird im Chinesischen ein neues Zeichen in der Regel ebenfalls aus der Komposition zweier schon vorhandener Zeichen, die 
ihrerseits oft als ,einfaches Bildzeichen' betrachtet werden. Und dabei fungiert das eine als ,Determinativ', das begrifflich eine Sachkategorie andeutet, und das andere als ,Phonetikum', weil dieses für die Schreibweise des neu zu bildenden Wortes, das (fast) homophon ist, herangezogen wird. ${ }^{14}$ Auch hier geht es um ein Doppelverfahren von Zergliedern und Kombinieren.

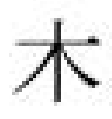

(a)

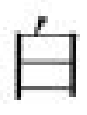

(b)

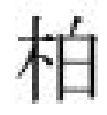

(c)

5 (a) „Baum“, (b) „weiß“ (a)+(b)=(c): „Birke“

Den Ausführungen von Natsume sei noch ein weiterer Aspekt hinzugefügt. Dank dieses ikonografisch-physiognomischen Verfahrens kann sich eine Manga-Figur flexibel variieren, ohne dabei ihre ikonische Identität zu verlieren. Auch in dieser Hinsicht ist sie mit einem Schriftzeichen vergleichbar. Die aus den Grundelementen zusammengestellte ,Protofigur' bildet also in sich eine grafische Einheit wie eine Glyphe. Diese variiert sich, um möglichst mannigfaltige Mienenausdrücke einer Person darzustellen. Eine solche Differenzierung könnte man wohl mit der grafischen Variierung der Glyphe in verschiedene Schriftarten vergleichen.

In jedem Schriftzeichensystem gibt es verschiedene Schriftarten wie romanische, kursive oder gotische im Alphabet. Aber zumal in der traditionellen ostasiatischen Kalligrafie wurde die Schriftart sehr früh schon systematisch differenziert. Bekanntlich hat die chinesische Schrift fünf Kategorien solcher Schriftarten: die Siegelschrift (䇡書), die Kanzleischrift (隸書), die Regelschrift (楷書), die Kursivschrift (行書) und die Grasschrift oder Konzeptschrift (草書). Diese Kategorien wurden teilweise auch im Hiragana aufgenommen und in der Kalligrafie weiter differenziert.

14 Vgl. Rolf Trauzettel: Bild und Schrift oder: Auf welche Weise sind chinesische Schriftzeichen Embleme? In: Werner Stegmeier (Hrsg.): ZeichenKunst. Frankfurt a. M. 1999, S. 130-163, hier S. 145. 


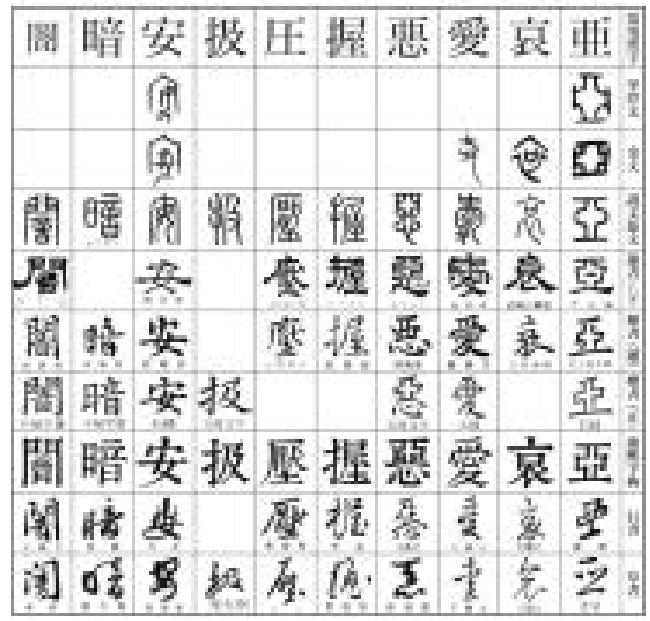

6 Chinesische Schriftarten

Vor dem Hintergrund einer solchen ostasiatischen Schriftkultur lässt sich vermuten, dass das von Tezuka entwickelte Methode sowohl für Manga-Zeichner als auch für -Leser umso leichter akzeptierbar ist. In diesem Sinne ist - so lautet die Schlussfolgerung von Natsume - das Manga-Bild wie ein japanischer Text lesbar: Personenfiguren werden im Prozess der Rezeption wie Kanji visuell bearbeitet, während zusätzliche Zeichen (Manpu) wie Hiragana gelesen werden. ${ }^{15}$

IV.

Gegen die hier skizzierte Fokussierung auf den ikonografischen Aspekt werden Einwände vorgebracht, und zwar von der Position, die bei der Thematisierung der Verwandtschaft zwischen Manga und der japanischen Sprache die Kontextabhängigkeit als gemeinsames Merkmal hervorhebt. Der Psychiater und Kulturkritiker Tamaki Saito (geb. 1960) ist einer, der diese Position vertritt und die ,zeichenzentrierte' Sichtweise kritisch in Frage stellt. Seine Ansichten sind es wert, hier eingehend erörtert zu werden.

15 Natsume: Manga no Chikara (wie Anm. 12), S. 196-203. 
Saito sieht das entscheidende Moment, das ein Schriftzeichensystem von einem anderen unterscheidet, nicht im Zeichen selbst, sondern in der Art und Weise, wie es kodiert wird - eine Ansicht, die auch etwa von Vilém Flusser vertreten wird. ${ }^{16}$ Und in diesem Zusammenhang kommt die Kontextabhängigkeit als Entscheidendes in Frage.

In seinem Buch über die Anime-Figur Sentoubishōjo (das kämpfende Mädchen) aus dem Jahr 2000 greift Saito Manga und die japanische Sprache gemeinsam unter dem Gesichtspunkt der Zeichenkodierung auf und definiert das Erstere als multikodierten Raum:

Manga ist in höchst eigenartiger Weise kodiert. Bekanntlich erfolgt die Code-Mitteilung im Manga durch mehrere Kanäle wie Bild und Sprache sowie zusätzliche Onomatopoetikon bzw. mimetische Worte. Und dabei ist das Bild selbst nicht einfach kodiert. Der Hintergrund [des Bildes] darf detailliert dargestellt werden, während die Figuren zeichenhaft vereinfacht gezeichnet werden müssen. Dies ist ein notwendiges Verfahren, um die bildliche Identität der Figuren in jeden einzelnen Bildpanels zu erhalten. [...] Mit dem Wort „zeichenhaft“ meine ich, dass hier die Darstellung selbst quasi als einfacher Code eine Botschaft direkt mitteilt, die ihrerseits mehrere Deutungsmöglichkeiten nicht zulässt. [...] Betrachtet man ein Panel im Manga genau, so stellt es sich sofort heraus, dass es multikodiert ist. [...] Der Ausdruck des Manga basiert auf einer ganzen Reihe verschiedener Codes. Vielmehr kann man sagen: Im Manga gibt es überhaupt keine Beschreibung, die „sinnlos“ da ist. Die Zeichnungslinien, die Aufteilung der Seite mit den Panels, sogar der Weißraum und die Auslassung - alle tragen zu einer Mitteilung der Bedeutung bei. ${ }^{17}$

Sicherlich ist Multikodierung kein Spezifikum für Manga, sondern gilt auch für Film oder Schauspiel. Die Eigenschaft des Manga liegt seiner Ansicht nach vielmehr darin, dass sie im Gegensatz zu diesen überhaupt keine polyfonischen Effekte hervorbringt. Hingegen funktionierten die verschiedenen Codes hier wie im Unisono. Sie wirkten nämlich nur dazu

16 Vilém Flusser: Alphanummerische Gesellschaft. (1989) In: Ders.: Medienkultur. Herausgegeben von Stefan Bollmann. Frankfurt a. M. 1997, S. 41-60, hier S. 43.

17 Tamaki Saito: Sentoubishōjo no Seishinbunseki (Schöne Mädchen kämpfen tapfer. Psychoanalytische Studie über einen Typ der AnimeFiguren). Tokyo 2006 ('2000), S. 277-279. 
zusammen, um eine einzige Bedeutung, eine einzige Stimmung oder eine einzige Situation mitzuteilen. Für diesen Zweck seien sie, synchronisiert‘.

Die Synchronisation der Codes korreliert Saito weiter mit dem rasanten Lesetempo des Manga: Je genauer und rhythmischer synchronisiert werde, desto schneller werde Manga gelesen. Das schnelle Lesen ist ihm zufolge ein Effekt eines solchen Unisono. Umgekehrt werden durch das schnelle Lesen die Mitteilungen der Codes umso leichter synchronisierbar. ${ }^{18}$

Das entscheidende Moment sieht Saito jedoch nicht in dieser synchronisierten Multikodierung. Entscheidend ist für ihn vielmehr die Tatsache, dass im Manga keiner dieser Codes allein vollständig funktionieren kann. Hier ist nämlich weder die Figur noch der Text in der Sprechblase noch das Hilfszeichen in der Lage, unabhängig von den anderen die Bedeutung eines Panels vollständig mitzuteilen. Alle Codes müssen daher komplementär zusammenwirken. Erst durch dieses Zusammenwirken kommt die Synchronisierung der Codes zustande, was einen UnisonoEffekt hervorbringt. Gerade in dieser Komplementarität der Codes sieht Saito die Voraussetzung für die hohe Kontextabhängigkeit des Manga.

Der Darstellungsraum des Manga ist in diesem Sinne semantisch überdeterminiert. Er ist daher einer der hohen Redundanz, die ihrerseits nichts Anderes ist als Effekt der geschilderten hohen Kontextabhängigkeit. ${ }^{19}$ Diese Redundanz hängt, wie bereits erörtert, einerseits mit der dem Manga spezifischen Zeitlichkeit, andererseits mit der hohen Geschwindigkeit beim Rezipieren eng zusammen.

Hieraus macht Saito auf eine vergleichbare Kontextabhängigkeit in der japanischen Sprache aufmerksam. Sie lässt sich auf verschiedenen sprachlichen Ebenen beobachten und geht eigentlich auf die oben erwähnte historische Hybridität der chino-japanischen Mischschrift zurück. Hier wird ein und dasselbe chinesische Zeichen für die Bezeichnung mehrerer Begriffe und Wörter von heterogener Herkunft in Anspruch genommen. Ein chinesisches Zeichen kann also in unterschiedlichen Weisen gelesen werden. Wie es wirklich gelesen wird, wird allein durch den Kontext, in dem es steht, entschieden.

Erweiternd kommt in diesem Schriftsystem eine große Zahl von Homonymen hinzu. Die Unterscheidung zwischen ihnen erfolgt dabei nicht auf Grund eines dem Wort zugehörigen Unterscheidungsmerkmals wie

18 Saito: Sentoubishōjo no Seishinbunseki (wie Anm. 17), S. 279. 19 Ebd., S. 280. 
etwa Genus oder Pluralform (diese gibt es im Japanischen nicht), sondern hängt wiederum allein vom Kontext ab. In der Schrift werden sie zwar dadurch voneinander unterschieden, dass sie bedeutungsgemäß mit unterschiedlichen chinesischen Schriftzeichen geschrieben werden. Es gibt jedoch auch viele Wörter, die mit denselben Schriftzeichen geschrieben werden, aber durchaus andere Bedeutungen haben. Hierfür ein Beispiel: Das Wort ninki (Popularität) wird mit denselben chinesischen Schriftzeichen (人気) geschrieben wie das Wort hitoke (Hinweis auf menschliches Leben). Das Erstere ist eigentlich ein chinesischer Begriff, während das Letztere von japaniser Herkunft ist. Der Kontext ist auch hier die einzige Instanz, die darüber Entscheidung trifft, welche von beiden beim Lesen aktualisiert werden soll.

Jetzt liegt es auf der Hand, dass es sich auch bei dieser Kontextabhängigkeit um ein komplementäres Zusammenwirken der zwei verschiedenen Codes handelt: einerseits der Kanji- und andererseits der Kana-Code. Und in der japanischen Kanji-Kana-Mischschrift ist weder Kanji (Bedeutungszeichen) noch Hiragana (Lautzeichen) imstande, als selbstständiges sprachliches Zeichen zu fungieren, wird doch ein Text erst durch die ,Unisono-Synchronisation' der beiden Codes überhaupt ,lesbar'.20

In einer solchen Doppel- bzw. Multikodierung sieht Saito eines der Hauptmerkmale, die die japanische Schrift von einer ,alphanumerisch ${ }^{6}$ kodierten (Vilém Flusser) unterscheiden. Flusser weist darauf hin, dass „flexionierende Sprachen“ „die Informationen zu Prozessen, agglutinierende zu Gestalten, isolierende zu Szenen“ verschlüsseln. ${ }^{21}$ Folgt man dieser Unterscheidung der drei Code-Typen, könnte man vielleicht sagen: In der Schrift des Japanischen als agglutinierender Sprache erfolgt die Verschlüsselung der Informationen in Richtung von multikodierten ,Gestalten‘.

Fassen wir zusammen: Sowohl im Manga als auch im japanischen Schrifttext teilen die Codes, einmal isoliert, die Informationen nur mangelhaft oder kaum mit, während sie, einander komplementär ,synchronisiert', durchaus in der Lage sind, überreichlich mitzuteilen. Dies ist die Parallele, die es ermöglicht, Manga so zu lesen, als wäre er japanische Schrift.

20 Vgl. ebd., S. 281-282.

21 Flusser: Alphanumerische Gesellschaft (wie Anm. 16), S. 43. 
V.

Von hier aus greift Saito das eigenartige Verhältnis des Manga zum Imaginären auf. Und dabei geht es ihm darum, die Ansicht, Manga sei wie die japanische Schrift an sich imaginär geprägt, kritisch in Frage zu stellen. Von ihren medialen Anfängen an tendieren Comics im Allgemeinen dazu, Fantasie oder Traumwelt zu thematisieren. Manga und Anime sind dennoch die Gattungen, in denen diese Neigung am deutlichsten zum Ausdruck kommt. Saito versucht das dargestellte Imaginäre im Manga aus dessen eigenartiger ,Zeitlosigkeit' ${ }^{\star}$ u erklären.

Im Anschluss an den Psychiater Hisao Nakai (geb. 1934) unterscheidet er die ,erlebte Zeit' (Kairos) von der messbaren, regelmäßig ablaufenden Zeit (Chronos). Im Manga und Anime wird die letztere, die eigentlich für die zeitliche Ordnung der Handlung einsteht, sehr oft durch die erstere ersetzt. Hier wird ein besonderer Augenblick quasi ,verewigt", indem er in mehreren Panels, sogar in mehreren Seiten geschildert wird. Somit wird in den Manga eine gewisse Zeitlosigkeit eingeführt. ${ }^{22}$ Saito weist darauf hin, dass der Manga die Neigung zu einer solchen Kairos-Zeit viel stärker hat als der westliche Comic oder Film, der medientechnisch dazu bestimmt ist, der Chronos-Zeitordnung zu folgen. ${ }^{23}$ Wie kein zweites Medium macht sich Manga zum Medium des Imaginären, indem er die Darstellungstechnik der ,Zeitlosigkeit' exzessiv in Anspruch nimmt.

Diese Zeitlosigkeit widerspricht der hohen Geschwindigkeit nicht, mit der Manga gelesen werden. In den, verewigten' Augenblicken nämlich, die die Zeitordnung der Handlung sprengen, erreicht die oben erwähnte Informationsredundanz ihre Höhepunkt. Diese Redundanz ergibt sich vor allem daraus, dass die Schilderung eines einzigen Augenblicks mehrere Panels und Seiten in Anspruch nimmt. Im schnellen Lesen wird das

22 Ein Beispiel für diese Zeitlosigkeit ist Astro-Kyüdan (Baseball-Team Astro) von Norihiro Nakajima (geb. 1950), ein Story-Manga, der 1972-1976 im Wochenmagazin Jump veröffentlicht wurde. In diesem Manga nimmt der Zeichner drei Jahre und über zweitausend Seiten in Anspruch, um ein einziges Baseball-Game zu schildern. Der Höhepunkt der Handlung wird auf diese Weise quasi grenzenlos verlängert.

23 Saito: Sentoubishōjo no Seishinbunseki (wie Anm. 17), S. 273. Im Film erfolgt eine solche Suspendierung der Chronos-Zeitordnung fast allein durch die Zeitlupenaufnahme. 
so Geschilderte vom Leser quasi als zeitlose, nämlich stillstehende Welt rezipiert. Rasender Stillstand - das ist die Welt des Imaginären, die in der Kairos-Zeit des Manga dargestellt wird.

Das Imaginäre, diesen bevorzugten Bereich des Manga, darf man jedoch keineswegs mit der Funktionsweise des Manga selbst verwechseln. Das Verfahren dieses Mediums ist - wie oben erörtert - durchaus schriftsprachlich kodiert und gehört daher eindeutig zum Symbolischen. Deshalb definiert der überzeugte Lacanianer Saito, der keine Verwechselung des Symbolischen mit dem Imaginären toleriert, Manga und die japanische Schrift gleicherweise als Medium, das uns „eine hochraffinierte Technik anbietet, mit dem Imaginären durch eine durchaus symbolische Operation umzugehen". ${ }^{24}$

Jetzt stellt sich die Frage: In welchem Verhältnis stehen der Ikonografismus und die Kontextabhängigkeit zueinander? Trotz des polemischen Arguments, mit dem Saito dem Ersteren die Bedeutung des entscheidenden Faktors absprechen will, schließen sich die beiden Aspekte prinzipiell nicht aus. Im von Tezuka entworfenen physiognomischen Verfahren wird das Zusammenwirken zumindest zweier Codes erwähnt: Figur und $\mathrm{Zu}-$ satzzeichen (Manpu). Insofern ist dieser ikonografischen Operation die von Saito zentral aufgegriffene Synchronisation mehrerer, jeweils nur unvollständig funktionierender Codes, wenn auch nicht explizit, vorausgesetzt.

Indes erscheint die Warnung Saitos vor gewissen ,Verwirrungen', wie sie eingangs erwähnt wurden, legitim, insofern es sich um die oft inadäquate Vereinfachung handelt, die die genannte imaginäre Neigung im Manga ohne weiteres auf die anscheinende Bildhaftigkeit der japanischen Schrift, zumal des Kanji zurückführen will.

Von seinem kritischen Standpunkt aus gesehen, kann man jene Tendenz in der ostasiatischen Schriftkultur, Schrift(-zeichen) als etwas Besonders zum Gegenstand eines Kultes zu machen, nicht mehr als Indiz für die unterstellte Affinität zwischen Kanji und dem Bildlich-Imaginären betrachten. Die traditionelle Kalligrafie, die nichts Anderes ist als ein hoch verfeinerter künstlerischer Ausdruck eines solchen Zeichenfetischismus, ist also ein Effekt der sprachlichen, aber auch kulturellen und sozialen Kodierung des chinesischen Schriftzeichens. Zum einen lassen Kanji an sich, solange sie sprachliches Zeichen sind, mehrere Lese- und Deutungsmöglichkeiten genauso wenig zu wie das Alphabet. Ihre verschiedenen Lesemöglichkeiten gehen nämlich auf die Kodierung der japanischen Schriftzeichenpraxis zurück. Zum anderen haben sowohl das Alphabet als auch Kanji und Kana

24 Saito: Sentoubishōjo no Seishinbunseki (wie Anm. 17), S. 282 f. 
in sich mehrere Schriftarten entwickelt. Diese Tatsache deutet umgekehrt darauf hin, dass eine zeichenfetischisierende Neigung kein Spezifikum ist, das exklusiv für die ostasiatische Schriftkultur gilt. ${ }^{25}$

VI.

Zum Schluss seien noch einige kulturtheoretische Überlegungen angestellt. Einem mediologischen Kulturvergleich, der Kulturen aufgrund ihrer Leitmedien zu klassifizieren unternimmt, wird die oft nicht unbegründete Skepsis entgegengehalten: $\mathrm{Ob}$ es sich dabei, wenn nicht um eine mediendeterministische, so doch um eine kulturtypologische Vereinfachung handelt, die leicht zu einer voreiligen, manchmal sogar ideologisierten Gegenüberstellung von Kulturen führt. ${ }^{26}$ Die kritische Position Saitos gegenüber der in der japanischen Manga-Diskussion dominierenden Tendenz, zwischen dessen ,Eigenartigkeit' und der des japanischen Schriftzeichensystems einen vermeintlich kausalen Zusammenhang automatisch aufstellt, geht offensichtlich von dieser Skepsis aus. Indem er seine Analyse um die Frage der Kodifizierung zentriert, verschiebt er das Thema vom Bereich der Medien auf den der Medienpraxis, also den der Kultur und distanziert sich somit von der mediendeterministischen Gefahr. ${ }^{27}$

Andererseits sei jedoch darauf hingewiesen, dass der von Saito beschriebene Raum des Manga dem im westlichen Japan-Diskurs wiederholten klischeehaften Japan-Bild zum Verblüffen ähnlich sieht - dem Bild jener Gesellschaft nämlich, deren organisatorische Prinzipien in der absoluten Priorität der ,harmonischen', d. i. gut synchronisierten Zusammenarbeit ihrer Bestandteile liegen. Hier wird auch synchronisiert, damit

25 Flusser vertritt auch diese Ansicht. Ihm zufolge werden die alphabetischen Zeichen, die auch hieroglyphische Elemente in sich haben, zu den Buchstaben, indem sie im prozessuellen Schema kodiert werden. Flusser: Alphanummerische Gesellschaft (wie Anm.16), S. 46.

26 So kritisiert Saito die „ungehemmte Idealisierung/Ideologisierung“ des Alphabets von Marshall McLuhan. Saito: Media ha sonzai shinai (Es gibt keine Medien). Tokyo 2007, S. 82.

27 Saito führt gegen die mediologischen Ansätze eine fundamentale Polemik. Seine These lautet dabei: Es ist unmöglich, Medien, die prinzipiell nur nachträglich gefunden werden, zum ontologischen Grund zu machen. Saito: Media ha sonzai shinai (wie Anm. 26), S. 7. 
ein Unisono-Effekt hervorkomme. Die Frage lautet jetzt, in welchem Verhältnis Manga, die japanische Schriftpraxis und die japanische Gesellschaft zueinander stehen - eine Frage, die den thematischen Rahmen des vorliegenden Texts nicht nur übersteigt. Versucht man, sie mit einem kausalen Modell zu beantworten, führt sie sogleich in die Irre.

Eine systemtheoretisch orientierte Betrachtungsweise wäre daher ein methodisches Gebot. Sie würde uns nicht nur erlauben, die Beziehung zwischen Manga, japanischer Sprache und der japanischen Gesellschaft aus der Perspektive gewisser Korrespondenzen differenziert zu beschreiben, ohne dabei das eine oder das andere als Determinierendes hervorzuheben. Aus dieser Perspektive wäre auch jene Tendenz in Japan, die Schriftzeichen in ihrer vermeintlichen Affinität zum Imaginären zu sehen, als kultureller Effekt der Schrift, nämlich als Reaktion des Systems Kulur auf seine mediale Umwelt, zu analysieren. Umgekehrt würde die Gefahr einer anderen typologischen Gegenüberstellung, die vielleicht im Argument von Saito steckt, noch klarer sichtbar.

In diesem Zusammenhang sei für das Konzept dieses Bandes, ,Kulturen von ihren Buchstaben her zu beleuchten', aus einer anderen Perspektive kurz noch plädiert: Versucht man, Kulturpraktiken der Gegenwart in ihrer Dynamik zu beschreiben, so kommt der ausgesprochen transkulturelle Prozess als Zentralaspekt dieser Praktiken in Frage. In diesem Prozess wird nämlich die kulturelle Differenz von Eigenem und Fremdem ständig und prozessual aufgelöst, was gleichzeitig die Genese bzw. Formation einer neuen Kultur bedeutet.

Aus dieser Perspektive erweist sich Grenzüberschreitung als entscheidendes Moment, durch das diese Kulturpraktiken geprägt sind. Und keiner wird es leugnen, dass Manga geradezu ein Kind der nicht abgeschreckten transkulturellen Grenzüberschreitung ist. Wie das Konzept einer Universalsprache bei Leibniz uns lehrt, kann die voreilige Identifizierung eines fremden Phänomens mit einem bekannten, die die kritische Position als Verwechselung vom Tisch weist, nicht selten Motor für solche kühnen Grenzüberschreitungen sein. Und bei jener, Verwechselung' des Symbolischen mit dem Imaginären wirkt eine Neigung mit, die in der Natur des Menschen tief verwurzelt ist. Es ist die Neigung, Sprachzeichen, dieses formale und daher in sich leere Zeichen für reine Differenz, nachträglich mit einem Sinn versehen zu wollen.

In den Vorlesungen von Roman Jakobson, die 1943-44 in New York gehalten wurden, lesen wir einen schönen Passus, der die Bedeutung dieser menschlichen Neigung für Kultur gegen den Formalismus dezidiert verteidigt: 
Contrarement à la thèse de Saussre, le lien entre le significant et le singnifié, autrement dit entre la série des phonèmes et le sens, est nécessaire; mais la seule liaison nécessaire entre les deux aspects, c'est l'association reposant sur la contiguité, donc sur un rapport externe, tandis que l'association reposant sur la ressenblance (sur un rapport interne) n'est que facultative. Elle ne se manifeste que dans les marges du lexqique conceptuel, dans des mot onomatopoétiques et expressifs [...]. Mais la question du rapport interne entre les sons et le sens du mot ne se clot pas là. [...] Nous avons dit que, tout en remplissant une function significative, les propriétés distincitives sont en elles-mêmes vides de signification. Ni une qualité distinctive prise en elle-même ni un faisceau de qualités distinctive, bref un phoneme pris en lui-même, ne signifie rien [...]: Or ce vide cherche à être rempli. L'intimité du lien entre les sons et le sens du mot donne envie aux sujets parlants de compléter le rapport eterne par un rapport interne, la contiguité par une ressenblance, par le rudiment d'un caractère image. ${ }^{28}$

Was Jakobson hier über die innere Beziehung zwischen dem phonetischen Signifikanten und dem Signifizierten sagt, können wir ohne weiteres auf den visuellen Signifikanten und das Signifikat übertragen. Der zwanghafte Drang dieser Sinngebung ist ein anderer Name dessen, was wir oben ,Zeichenfetischismus' genannt haben. In diesem Sinne haben, Verwechselungen' und ,Verwirrungen' über Manga und die japanische Sprache auch das Recht, legitime Gegenstände einer kulturwissenschaftlichen Analyse zu sein.

\section{ABBILDUNGSVERZEICHNIS}

1-3 Osamu Tezuka: Manga no Kakikata. Tokyo 1956, zit. in: Fusanosuke Natsume: Manga no Chikara (wie Anm. 12), S. 200-202.

4 Takao Saito: Golgo 13, Bd. 75. Tokyo 1990, zit. in: Inuhiko Yomota: Manga Genron (wie Anm. 2), S. 189.

5 Ryozo Maeda: Rasender/Lesender Stillstand (wie Anm. 4), S. 209.

6 http://tonan.seesaa.net/article/20516478.html.

28 Roman Jakobson: Six leçons sur le son et le sens. Préface de Claude Lévi Strauss. Paris 1976, S. 118. 



\title{
THE AESTHETIC CHARACTERISTICS OF KANA SEEN IN CHIRASHI-GAKI
}

\author{
INTRODUCTION
}

I was given the opportunity to join a series of workshops at Morphomata. China and Japan both have well-established cultures of calligraphy, so one of the goals of the workshop was to explore the elements common both of those cultures, while at the same time introducing as much of their contrasting features as possible. For the demonstration part of the workshop, the initial plan was to take up the topic of brush strokes. I went about preparing with that intention, but as I thought back, I realized that my interests were drawn to features that I had not anticipated. What is more, I came to realize then that those features might be rooted in the essence of Japanese calligraphy, or to put it differently, what makes Japanese calligraphy Japanese.

I ended up spending most of the actual presentation on styles of calligraphy -Chinese calligraphy in particular-whose emphasis is on characters. The reason is that before even touching on the topic of Japanese calligraphy, I decided it was necessary to go over certain principles and concepts that are key to developing an understanding of the history of Chinese characters, calligraphy in China, and the unimaginably rich cultural traditions they represent. Nevertheless, it occurs to me now that what captured the most attention during the demonstration was not the specimens of calligraphy in kanji (Ch. hanzi), with their trinities of shape, pronunciation, and meaning, but rather the appearance of kana, characters which in themselves lack any conceptual meaning. This was probably in part because my practical experience and degree of technical prowess lend themselves more to kana than kanji. But even if one were to make allowances for that, there seems to be some measure of value in 
exploring the pure aesthetic qualities perceived by people of cultures not intimately familiar with these writing systems. In this paper, I build upon my past studies of kana, draw upon those experiences at the workshop, and discuss the aesthetic characteristics of calligraphed kana, particularly as seen in chirashi-gaki. ${ }^{1}$

This discussion bypasses the conventional first step of talking about how the characters came about. Instead, the focus is placed on how one engages with the characters when doing calligraphy. When one considers the matter of the aesthetic characteristics of calligraphy, what one is concerned with are not the characters themselves. Nevertheless, the issues that arise can never entirely be separated from characters. Issues such as the postwar trend towards more refined strokes in calligraphy and the role and nature of characters in the composition of calligraphic works have arisen because they involve writing as a historical product of calligraphy. To explore that relationship, one must look at issues such as how calligraphers engage with characters and how characters manifest themselves. This paper will discuss characters in the context of how the experience of calligraphers intersects with characters as historical artifacts, and examine the origin of characters insofar as it relates to this theme.

\section{HOW TO NOT WRITE}

Chirashi-gaki is a style typical of kana calligraphy in which the calligrapher does not align line heights or spaces. It is said to originate in the writing of personal letters (shōsoku), where authors were prone to write spontaneously and impulsively, resulting in unintentional uneven lines.

1 The current paper is a substantial revision to the following works with changes made to reflect the particular themes explored here. Noriko Kaya: Chirashi-gaki'-kō ("A study of chirashi-gaki”). In: Kambayashi Tsunemichi (ed.): Nihon no Geijutsu-ron: Dentō to Kindai ("Japanese Artistic Theory: Tradition and Modern”). Minerva Shobo 2000, p. 153-173; Noriko Kaya: Mizukuki no 'yawarageru' chikara: Kana no sho no biteki kachi ni kansuru ichi-kōsatsu ("The power of the brush-written word to 'soften': An examination of the aesthetic values of kana calligraphy"). In: Shogaku Shodoshi Gakkai (eds.): Shogaku Shodō-shi Ronsō("A Collection of Papers on Calligraphy and Calligraphic History") 2011, p. 218-421.-Translated titles of Japanese works in this paper are intended strictly for reference purposes. In no way do they imply the approval of the authors or the respective accuracy thereof. 
The beauty perceived in the asymmetry produced by an unfettered brush gradually became formalized as a composition technique. ${ }^{2}$ Here it is important to take particular note of two conventions that came to be a part of formalized chirashi-gaki: one is the practice of visually representing intervals, and the other is the art of subtly revealing the significance of blank spaces that is ordinarily repressed or hidden in everyday writing.

One of the most important attributes that one must be mindful of in order to master this style is space-as in blank space. It goes without saying that there is an internal consistency to brush strokes and the use of space that characterizes every style of calligraphy, but one of the most salient stylistic differences that separates kana from kanji-and more pertinently chirashi-gaki from other writing styles of kana written in ordered, aligned, and symmetric lines-is the attention given to what is not written.

For example, if a calligrapher begins a line high up on the paper, the calligrapher will go about writing that line while simultaneously making decisions about how far down to end it, or conversely how much space to leave at the end, where to start the second line, and how bold it should be. The decisions continue in the second line, when determining where to start and finish with consideration of its balance with the first line. The 'relationship' that forms between the written lines involves such elements as the size of the characters, the thickness of the strokes that make up the individual characters, the distance between them, the relative shape and size of adjacent characters, the amount of ink contained in the bristles of the brush, etc. What gives life to this relationship is the internal consistency of the brush strokes. The calligrapher is aware that the brush strokes are defined by the features that repeat themselves and must be sustained throughout the work. The calligrapher's aim is for the changes in the speed and urgency with which those continuous elements are drawn to appear as variations in shape and form in the end result.

2 The predecessor of the term chirashi-gaki is found in Yühitsu Jōjō (The Chronicles of the Chronicler Jō), an esoteric work entrusted to Akita Jnosuke and Adachi Yasumori by Sesonji Tsunetomo in the year Bun'ei 12 (1275): “書仮名色紙様。大略者同真名色紙形。其内或草或普通二低昂テ可被 散書 ('The style of writing kana on decorative paper is largely the same as writing kanji on decorative paper, but one should vary the [line] heights and write hurriedly[chirashi-gaki] when writing sōgana or hiragana')", where it says that one should write in “書 (chirashi-gaki)" when writing kana on shikishi. 
The shapes formed by the characters have an inherent diversity by virtue of just being two-dimensional, but unless those shapes resonate with each other, the space present has no tension to it. A single stroke viewed out of context might look frail and feeble, but within a work it can impart a sense of flowing speed within the work depending on its placement. The areas around it where nothing is written are what bring attention to the features of the stroke. A character that, fixed to the paper, seems to embody a certain action, and might be likened to a single frame of cinematic film. But what makes chirashi-gaki so fascinating is not just the temporal quality that results from those frame-like brush strokes appearing in succession, but also the fact that a single brush stroke can have an effect on the appearance of an adjacent line or another stroke some distance away from it. In that sense, there is a certain 'drama' to works of chirashi-gaki, and by discovering the relationships present among the various elements within, one can is able to experience multiple modes of appreciating the work.

In formal chirashi-gaki, the calligrapher is confined to the limited amount of space offered by the sheets of paper that are conventionally used. As a consequence, from the perspective of composition, the calligraphy has to exist as a complete microcosm that is perfectly self-contained on a single sheet of paper. That said, this aesthetic microcosm is not created by characters merely being arranged inside the predetermined confines of the paper. The elements of expression linked organically to one another through the act of writing require as a matter of necessity a certain amount of space for the writing process. Put differently, the act of writing is governed by absolute spatial constraints, but at the same time, the size of the space is determined in the process of the brush running along the paper. With calligraphy in the chirashi-gaki style, the visual dynamism generated by concord among groups of characters and connecting strokes imposes order between lines of characters. This order connects dynamically with other aesthetic elements only because the limited space allowed by the paper causes blank space to take on artistic value that is more than passive. This space represents shape that is never at the forefront of the layout, and in this sense it serves as a background; however, if one takes note of the fact that this kind of space is created at the same time as lines are drawn-that lines and space are inextricable-one sees the significance of its latent potential. 


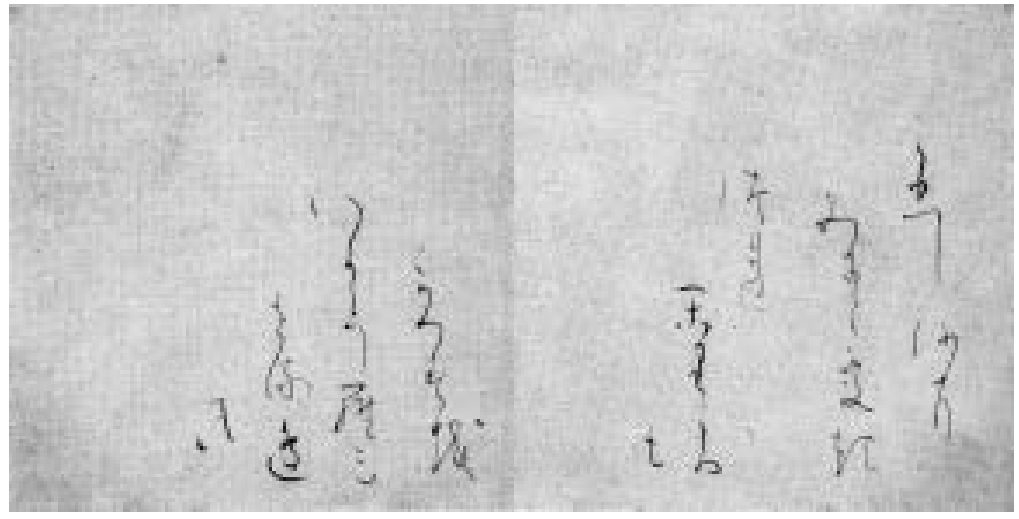

1 Fragment known as "Tsugi-shikisi". Traditionally attributed to Ono no Tôfu, $13.2 \times 26.6 \mathrm{~cm}$, Heian period (second half of the $10^{\text {th }}$ century)

\section{EXPRESSING STRUCTURE AND SPACE: TSUGI-SHIKISHI (FIGURE 1)}

\section{II.1. COMPOSITION}

Let us look at a sheet of calligraphy from a classic work and see how these concepts actually play out. This example of calligraphy is one sheet from Tsugi-shikishi, which is an archetypical example of the chirashi-gaki style. Tsugi-shikishi was originally compiled as a single bound volume of mid-Heian waka poems written in different spatial layouts. With the development of the tea ceremony in the Muromachi period and later, the way that people enjoyed calligraphed poetry began to change. Specifically, it became increasingly common to remount the sheets of calligraphy onto hanging scrolls or into albums and enjoy them individually. This practice is discussed in more detail in a later section in connection with the development of kire (cuts or fragments). In the present section the focus of the discussion will be on the shapes that occur in an extant article of calligraphy from Tsugi-shikishi and their significance.

This sheet presents a relatively even composition, with a large amount of space left at the top of the horizontally oriented paper and the 'feet' of the lines fairly well-aligned. The arrangement of the lines forms two large groups. In terms of groups of characters, the five half-lines up to “閑里ころも (karikoromo)" on the right page of the decorative sheet make up one group and all the characters after that make up the other. The end of the first half also coincides with the cutoff point for the first half of 
the poem. Judging by the number of the characters and the complexities in terms of form, there is a kind of motion present on the sheet in which the first half is dominant and the second is subordinate. This compositional feature, however, can only be observed when the sheet is viewed all at once in its entirety. Diverse groupings are formed through visual perception in the sense that they change depending upon how the eye of the observer registers such things as where the calligrapher has re-dipped the brush, the number of characters per line, and the starting positions.

\section{II.2. STROKE FULLNESS AND INK TONE}

The volume of ink present in the first line, which begins at a higher position than the remaining lines, gradually decreases as the brush progresses to the bottom of the line. This change becomes even more evident as the calligrapher goes from the first line to the second and from the second to the third. Nevertheless, within each line one can see places where there is more ink in a character than in the one that immediately precedes it. The same applies to individual strokes. Stroke fullness is not merely a matter of the volume of ink in the brush progressively decreasing. Rather, it is related to a number of variables, including the speed of the stroke and the amount of pressure applied to the paper with the brush. Changes in stroke fullness impart a sense of visual continuity as well as an almost three-dimensional quality. Here, the flow that was created from the first through the third lines is temporarily interrupted because the calligrapher has replenished the ink at the beginning of the fourth line. Note, however, that it is an interruption, and not a cessation. The beginning of the fourth line is located close to the terminus of the third line, and the visual rhythm of the third line is carried unbroken into the fourth. Hence, because the calligrapher re-dipped the brush and began the fourth line at that particular location, a new visual group is established that extends from the third to the fifth line.

Taking a more careful look at the flow of the lines, it is evident the center of nearly all of the lines shifts to the right. This is very much in keeping with the principles governing brush strokes when joining characters together. Among the many styles of handwriting, renmen, or joined writing, is an abbreviated writing technique born out of the need to write quickly. Given the way the brush is used when writing Japanese, maintaining joining strokes in a straight, perpendicular line and positioning characters directly under the characters that precede them is unnatural. Ordinarily, the last stroke of a character tends to get omitted as one writes, thereby shortening the path to the succeeding character. As a result of 
the ink application method used as well as the shift of the line centers, “徒連 (tsure)" in the third line is part of both the group formed by the first three lines and the group formed by the last three lines on the right page of the sheet. This results in a dynamic overall composition.

The last line of the right page consists of the single character " $\theta$ (mo)". As the calligrapher wrote the preceding four lines, the center of each line shifted towards the right, causing the visual dynamics of the whole of the right page to slope downwards and to the right. The result is that the space produced on the left of the right page appears to the observer to encompass a larger physical space than it actually does. The area where nothing is written counterbalances the area occupied by writing. The sense of evenness in the composition of the right page is achieved by the character "も" of the last line through the use of this blank space. Used in such a manner, blank space takes on the significance of artistic space.

The replenishing of ink at the beginning of the fourth line on the right page likewise serves to form a small visual group comprising the fourth and fifth lines. This small group is situated along the imaginary gently waving line formed by the beginning of the left page, and is also contained within the curve formed by the feet of the lines on the right page. The "も" of the last line of the right page is incorporated in the space in the upper left-hand portion of the right page and generates a visual continuity with the tops of the first two lines on the left page. The sharp rightward slant of the first line on the left page has an even stronger effect on the space spanning both pages. The second line of the left page, with its somewhat high starting point and gentle rightward slant, serves as a buffer that softens the sharp rightward slope of the page's first line. The concord among the features does not end here. The gentle rightward slant of the second line of the left page transforms into a leftward flow beginning with the character “羅 $(r a)$ " (fourth character, second line, left page). The swell of this line is balanced out by the character “き $(k i)$ ", which concludes the left page as well as the whole work.

When looking at the work in this light, one can see that what connects the left and right pages are the lengths of the individual lines, the positions at which they begin and end, the spaces in between them, and the degree to which they slant.

Let us take another look at the work as a whole. There is a large physical space present at the junction of the left and right pages. If one focuses one's attention on the way the ink is applied and the way the lines are structured, one can see that this space forms another composition, 
one in which the dominant and subordinate parts are reversed, i.e. the first line to the third line on the right page becomes the subordinate part and the fourth line on the right page with the entire left page becomes the dominant part. The ink is applied in a way that makes physical space a part of the composition and helps bring about a sense of visual continuity.

\section{II.3. CHARACTER USE}

In the above, the discussion of this work has centered on the structure of lines and use of ink. In order to maximize the aesthetic effect of those techniques, one must focus on the characters used.

For example, in order for the pair of characters “徒連” in the third line of the left page to act in concert with the second line to form a group, they must have a natural rightward slant. The choice of “徒”, which has a square shape to it, as the first character of the third line, and the vertically slender “連” as the second, effects a degree of undulation in the second line and closely links it with the lines on either side. If instead the calligrapher had used as the first character of the third line "つ" instead of “徒” to represent the syllable $t s u$, there would inevitably have been a stylistically undesirable repetition of shapes with the clockwise-swirling line in “夕 ( $m i)$ ", which is the first character of the preceding line. Likewise, if he had chosen the character “れ” instead of “連” to represent re, there would be no rightward flow to the third line.

We shall now turn our attention to the use of "羅” (fourth character, second line, left page) and surrounding characters. In the second line of the left page, the simplicity of “う (u)" (third character) is counterbalanced by the complexity of “羅” (fourth character), which also shifts the center of the line to the left. Because "羅” is relatively square-shaped, even though the center of the line slants leftwards, it is still in concordance with the line to the right of it. The character “春 $(s u)$ " located to the right of "羅” is vertically long. Furthermore, the first line of this page exhibits a strong rightward slant. If, for example, a simple character like the character " $(r a)$ " that is more vertical in shape were to be used as the fourth character of the second line, the first and second lines would be visually discordant. The use of "羅" as the fourth character of the second line ensures that the resulting shape has an effect on the space between the second and third lines, as well as enhancing the effect of the replenished ink on the brush in “邊 (be)” (third character, third line, left page).

The characters chosen for the feet of the lines on the left page are “越 $(w o)$ ”, “三 (mi)”, “邊 (be)”, and “き $(k i)$ ”. A pleasing balance between the sparse and dense is produced along in the feet of the lines as a result 
of alternating characters of complex shapes with those of simple shapes. There is also a connection between this pattern and the characters “耳 (ni)”, “起 (ki)”, “ろ(ro)”, and “も (mo)” comprising the feet of the lines on the right page.

All of the factors above work in concert and aesthetically affect one another. The factors that affect the quality of a calligraphed waka poem include the syllabic characters chosen, the brush techniques used to join those characters, and in the case of different characters used to represent the same kana, the individual features that make up that character, such as the length and angle of each stroke. With Tsugi-shikishi, which uses a mixture of sogana and hiragana, the correlations among these factors are organic. In this respect, the character as a unit is broken up into its constituent parts, and the 'symphonic' correlations that arise between individual brush strokes manifest themselves as visually dynamic relationships.

\section{THE TRANSFORMATION OF SPACE}

\section{III.1. KIRE}

As demonstrated in the above, it is possible to analyze the relationships among manifest shapes. These shapes represent spatial transformations that occur within the small confines offered by sheets of paper. However, such transformations were not completed in the contextual vacuum of single sheets of paper but evolved over time. The following is a discussion of how changes in the ways in which people were exposed to and enjoyed calligraphy led to modes of presentation on sheets of paper such as those described above.

Many articles of classical calligraphy were originally part of long scrolls and booklets, but later came to be cut out for viewing purposes and mounted to hanging scrolls or pasted into albums called tekagami (collections of exemplars of classical calligraphy). This is how kire (cuts or fragments) came about. Tradition holds that the practice of kire began when Muromachi-era tea master Takeno Jōo recognized the essence of the tea ceremony in essays on poetry by Fujiwara no Teika, and as a result hung samples of Ogura-shikishi calligraphy (i.e. calligraphy by the hand of Teika himself) in his tearoom. After this, kire grew in popularity in conjunction with the rise of the tea ceremony in the Muromachi period onwards. Articles of calligraphy hung on walls had to meet high standards in terms of poetic content, skill with the brush, aesthetics of 


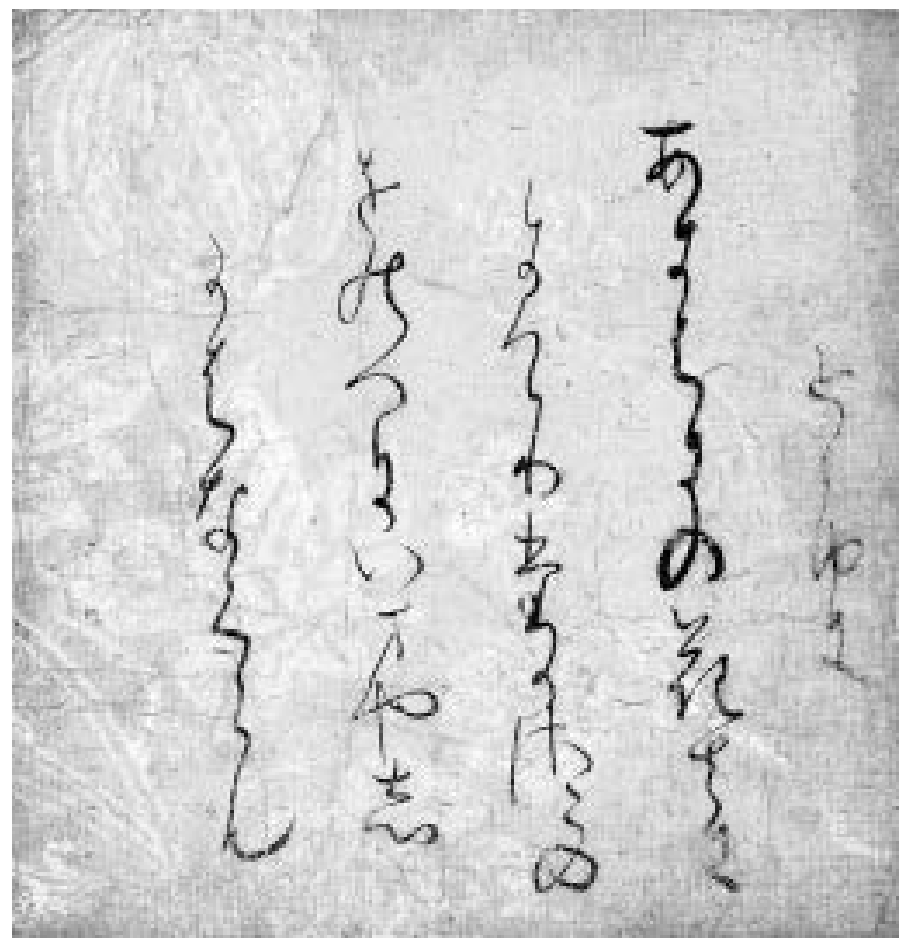

2 Fragment known as "Sunshōan-shikishi". Traditionally attributed to $\mathrm{Ki}$ no Tsurayuki, $13 \times 12.3 \mathrm{~cm}$, Heian period (end of the 11th century)

the decorative paper, and more. The present section is an exploration of the ways in which the significance of calligraphy changed and grew over time with the advent of kire, particularly as it relates to the issue of space in chirashi-gaki.

In terms of cultural history, the development of kire calligraphy brought about new modes of viewing calligraphy in addition to a rediscovery of classical calligraphy in general. Still, aesthetic experiences with calligraphy differed depending on what bound works ended up as kire.

In the case of Sunshōan-shikishi (Figure 2), where the calligraphy therein was originally bound in one booklet, the medium prompts one to ask just what blank spaces around the brush strokes constitute space that is essential to the work. The act of cutting out a piece of calligraphy and framing its boundaries determines the latent artistic potential of the blank space. The resulting article may have beauty in its brush strokes 
as well as the beauty in its use of space. ${ }^{3}$ In such cases, later generations of calligraphers will have added to the aesthetic quality of the work in terms of both brush stroke and space.

However, even if the work was part of a bound booklet, when separated from the whole and turned into kire it could present a spatial composition that is wholly different from when the work was penned. The original binding of Tsugi-shikishi was such that the sheets of decorative paper were folded in two and came into view when the booklet was opened. The fold in the middle corresponds to the margin or "gutter" left from the glue-bound spine. Poems were divided into couplets, with the first and second stanzas appearing on separate pages. In most cases this style meant arranging waka poems so that they could be read in their entirety when one opened the booklet (i.e. on opposing pages), but there are also cases in which the first stanza appeared on the left page and the second stanza appeared on the following page, i.e. the right half of the following sheet (called watarigaki; Figure 3). In the latter case, a bound book was taken apart and the two pages comprising one waka poem were spliced together into one sheet. Tsugi-shikishi, which literally means 'spliced colored paper', gets its name from this practice. This specimen

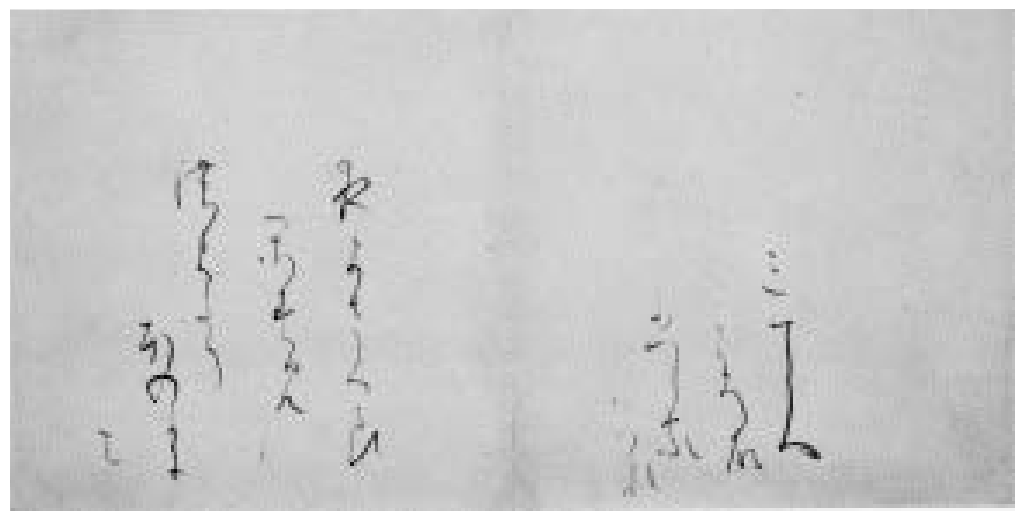

3 Fragment known as "Tsugi-shikisi". Traditionally attributed to Ono no Tôfu, $13.2 \times 26.6 \mathrm{~cm}$, Heian period (second half of the $10^{\text {th }}$ century)

3 Because extant specimens have been restored to repair insect and other damage, the (restored) blank spaces seen now are not, in some cases, the same as those that were present when the practice of kire was established. 
was made into kire in 1906, long after it was penned. The pages therein are thought to have consisted not only of layouts in which the left and right margins were the same, but also of layouts in which the margins were offset left-to-right and top-to-bottom (Figure 4).

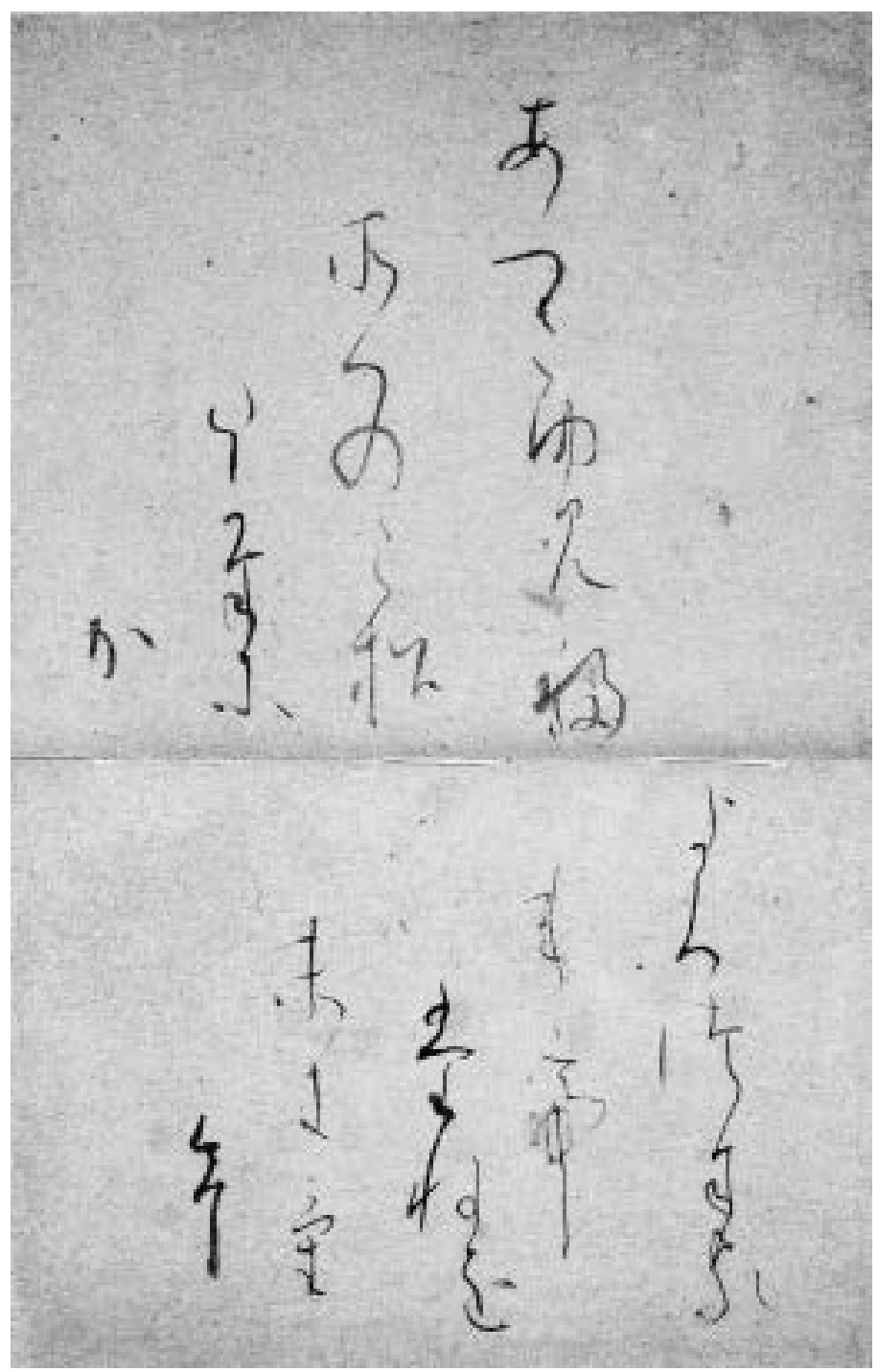

4 Fragment known as "Tsugi-shikisi". Traditionally attributed to Ono no Tôfu, $19 \times 12 \mathrm{~cm}$, Heian period (second half of the $10^{\text {th }}$ century) 
In what ways did the spatial composition take on new significance when the volume was taken apart compared to the spatial composition when the Tsugi-shikishi was written? One thing that one must keep in mind is that all of the pages contain just half a poem, and that the orientation of the binding was watarigaki. In the booklet, a single waka poem is written over two pages, so the visual image of the succeeding passage is pushed to the next page. The sense of anticipation on the part of the observer is heightened due to the fact that part of the waka is written on a page that cannot be viewed simultaneously. This style of viewing is predicated on the continuation of poems across more than one page. What is more, the spatial composition is interchangeable because only half a poem is written on a single page. Put differently, in the watarigaki portions of the booklet, the observer visually takes in a double-paged composition (in which the right page features the second stanza of a separate poem), but at the same time there arises in the mind of that observer a visual image of what should come next on the right side of the following sheet (corresponding to the second stanza of that poem). The very fact that the succeeding page cannot be viewed concurrently makes the viewing experience more dynamic, as it enables the observer to contemplate the interchangeable possibilities of spatial composition.

One can see in Tsugi-shikishi not only undeniable relationships that are self-contained within each sheet of the work, but also a continuous order formed from page to page. For that reason, new spatial compositions created by later generations should not be thought of as separate from the act of writing, but as receptacles for it.

Let us now look at an example of a calligraphic work, namely Koya-gire (Figure 5), that was originally compiled in scroll form. The orientation of scrolls means that there are not preset limitations to the amount of space that can be used. The volume of space occupied by text grows longer or shorter in size depending upon how much the calligrapher writes. Works in scrolls mostly come into existence through the development of constituent parts. One reads or appreciates scrolls by unfolding them in the same order in which they were written. Scrolls are not written in a way that would allow someone to view the entirety of the work all at once. When a portion of text is cut away from a scroll, it is up to the person doing the cutting to decide on what part and how much needs to be cut, a decision which in turn determines the differences in spatial composition between the original work and the cutout portion. It goes without saying that characters themselves do not change, but character 
groupings, line compositions, ink application, and other intricately related aesthetic factors may very well change, so much so that a difference in just one line in the cutout part can drastically change its appearance. To turn a portion of a scroll into kire is to impose spatial confines upon an otherwise continuous area of space. This act marks the point at which composition is born.

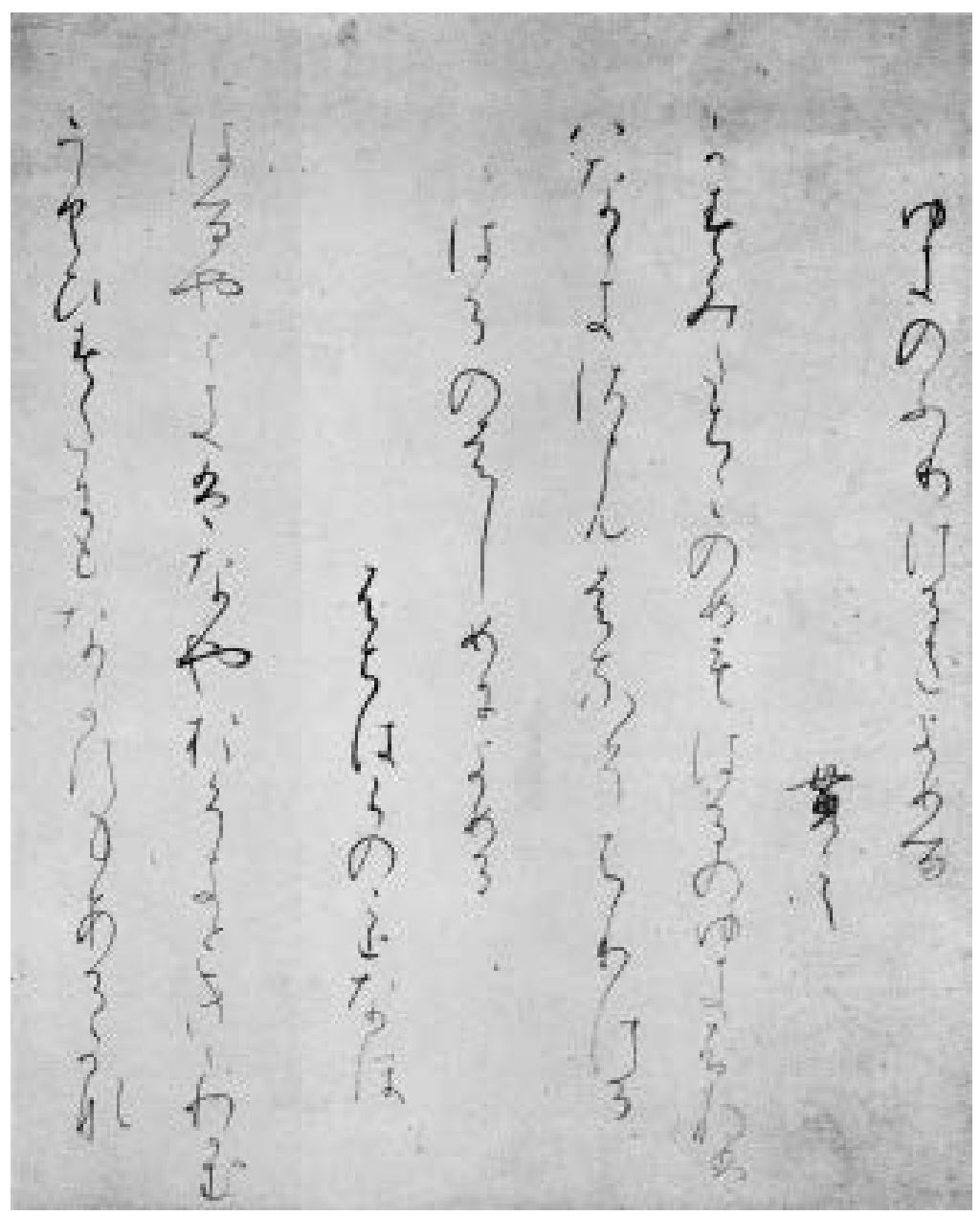

5 Fragment known as "Kōya-gire". Traditionally attributed to Ki no Tsurayuki, $26.3 \mathrm{~cm}$, Heian period ( $\mathrm{mid} 11^{\text {th }}$ century) 


\section{III.2. KIRE-TSUZUKI (CUT-CONTINUITY) AS A CHARACTERISTIC OF JAPANESE AESTHETICS}

In 'Kire' no Kōzō, Ryôsuke Ôhashi has this to say about the essence of what beauty is in Japanese aesthetics. "Kire-tsuzuki (cut-continuity) refers to a construct in which disparate things encroach upon one another but stand out as entirely different entities." If one looks again at the fragments of classical calligraphy examined above and view them as manifestations of kire in the context of Japanese writing culture, one can see with greater clarity the significance of the brushstrokes and shapes that occur in kana calligraphy.

Ôhashi expands upon the essence and potential of kire.

Above all, 'kire' becomes apparent wherever the subject himself 'cuts out' his own mundane nature and attains an awareness of himself. There is another, deeper meaning to the word 'kire'. This second sense of the word corresponds to 'nature' in its second sense, namely, 'nature' as in the 'true nature' of things or people, a sense which derives from 'nature' in the sense of 'natural surroundings'.

In Japan and China, where 'external nature' and 'internal (or true) nature' are not seen as opposing viewpoints, nature has conventionally been viewed as 'that which is so of itself', and the dichotomized, dual meaning of the word has only come to be recognized in modern times. ${ }^{6}$ Following this line of reasoning, it seems more likely that one would find direction for the modern and post-modern world among the possibilities of kire in its pre-modern conception. Those possibilities "lie in the unique relationship between 'nature' and 'art' that go beyond kire [...] The very 'nature' that was 'cut out' through art lives reborn within that art." The aesthetic domain described here is where natural beauty and artistic

4 Ryôsuke Ôhashi: 'Kire' no Kōzō: Nihon-bi to Gendai Sekai ("The Structure of 'Kire': Japanese Aesthetics and the Modern World"). Chuokoron-sha 1986, p. 85 .

5 Ibid., p. 285.

6 On the concept of nature in calligraphy, see Noriko Kaya: Shogeijutsu no Chihei: Sono Rekishi to Kaishaku ("The Horizon of Calligraphic Arts: Their Hisotry and Interpretations"). Osaka 2000.

7 Ôhashi: 'Kire' no Kōzō: Nihon-bi to Gendai Sekai op. cit. (note 4), p. 287. 
beauty merge. To use ikebana (flower arrangement) as an example, 'art' is cutting a flower in such a way that the (true) nature of the cut flower is brought back to life.

How have nature and art interacted in calligraphy, which as a matter of course involves the human artifact of writing? Let us again consider the mindset of the calligrapher in the midst of calligraphy. The artistic awareness needed to ensure that characters complement each other or that blank space is used effectively is inextricably linked to the pursuit of visual effects in the most basic sense of the term. When a calligrapher views the cropped sheet of paper as a microcosm and tries to compose characters tightly within, it is essential not to perturb the structure of the characters; on the other hand, if approached with a structuralist frame of mind, the desire to create shapes could lead the dissolution of the characters. The ideal of calligraphy is not to seek the right number of strokes or the necessary lengths to make characters sufficient to function as symbols, but instead to draw characters in a way that naturally fulfills the requirements to function as symbols. In other words, whether calligraphy is aesthetically pleasing depends on whether it captures what one might call the true nature of characters. When one seeks out this true nature, one will begin to see the importance of imitating the brush strokes of the pioneers of calligraphy. Put differently, the only way one can know if the true nature of characters is being captured or not is to actually write them. Copying the works of masters (known as rinsho) is an important act that has always been practiced in the world of calligraphy. Through the act of practicing brush strokes, one explores and masters the true nature of characters. In the same vein, understanding the true nature of characters is to discover them as historical shapes.

The most important aspect of brush strokes in kana calligraphy is the how the strokes are connected and severed in renmen (joined writing). A single kana syllabogram by itself has no conceptual meaning. It is only when two or more are strung together that they form words and sentences. Given this linguistic utility, the significance of brush strokes is not readily apparent in everyday writing. One must learn through repetition where and how to sever strokes and where to join them up again, and in the process, one becomes more aware of the above-mentioned spaces where 'nothing is written'. 


\section{THE NATURE OF BEAUTY IN KANA}

\section{IV.1. THE FORCES LEADING TO THE EMERGENCE OF RENMEN}

The following is a discussion of how renmen came to be. The linguistic features of kana contributed greatly to the spread of renmen as a custom and to the development of renmen techniques that connect two or more characters at a time. ${ }^{8}$ Writing depends on the use of words with some level of cohesion, rather than just individual characters. As one kana syllabogram represents one syllable, the smallest unit that requires brush strokes is a word. The earliest signs of a joined writing system can be traced back to the borrowing of forms of kanji for use in Japanese. Hiragana, which developed out of man'yogana as the latter was increasingly written in flowing styles, came to be abbreviated to such a degree that they could no longer be recognized as the kanji from which they derived. Written with subdued strokes, hiragana evolved into a soft and curvy script. Renmen was developed out of the need for a way to write words quickly, easily, and with efficient movements. The brush stroke patterns were applied directly to the continuous motion of the strokes, resulting in styles of writing that flowed freely and naturally from the hand, which constituted the basics of calligraphy.

"Letters written spontaneously about profoundly wistful thoughts of the transience of things are well worth seeing（もののあはれに思 ひながさる〉ま〉に、筆にまかせて書きながされたるは、みど ころあり).”

8 "There is not one example of 'renmen-s' to be found among works with kunten (guiding marks for reading Chinese) in hiragana. Hiragana as seen in old documents and kana works are very different in this respect. Hiragana in old documents and kana works was an unfiltered expression of 'pure' Japanese writing, but kana in kunten works, on the other hand, were, speaking in extremes, just pronunciation notations of a sort, put there as notations giving kanji readings in Japanese" (Hiroshi Tsukishima: Heian Jidai no Kana to Kotoba ["Kana and language in the Heian period"]. In: Shodō Kenkyū ["Calligraphy Research"]. Kayahara May 1990, p. 52).

9 Atsuyoshi Sakakura (ed.): Yoru no Nezame ("Wakefuless at Night"), Vol. 1, Nihon Koten Bungaku Taikei ("Collection of Classical Japanese Literature") 78 (1964), p. 61. 
This passage is from a work of narrative literature in which there is shosoku (a personal letter written in kana) saying that well-received calligraphy is that which is written spontaneously about deeply felt emotions. There are two important perspectives to note here. One is the clear flow of each line, and the other is the undulations present in that flow. Shossoku were written in conversational Japanese, and the mood and sentiments of the author are in no small measure reflected in the brushwork of the letter. If writing prose, the author would try to write coherent units of language such as single words or phrases all at once. In addition, shōsoku are normally longer than waka. Punctuation marks are not used in shōsoku, so the rhythm of the language is conveyed through breathing techniques in the joined writing and the density of spaces between characters. ${ }^{10}$ The emotions of the author are apparent not only from what is said in letter but also in the brush strokes. ${ }^{11}$ In letters, brush strokes are important as a medium for conveying the author's mood and sentiments.

The writing of shosoku and waka is rooted in the uncontrollable desire to articulate one's inner thoughts. ${ }^{12}$ When written Chinese (written in

10 The rhythm in terms of language is closely tied to the breathing of the author. This relationship has been noted before in ancient texts created after man'yogana texts: "Once prose is composed in hiragana, there are generally no hints left for dissecting and deciphering strings of kana other than the speed with which they were written as seen in the ink re-dipping, joined writing, the positioning of characters, etc." (Takashi Inukai: Jōdai Moji Gengo no Kenkyū [“A Study of Ancient Japanese Writings”]. Kasamashoin 1992, p. 317).

11 The passage describing the response to the shosoku from the emperor is as follows: “萩がさねの紙に、もるからにあささぞ見ゆるなかぐ にいはでをやみ水岩がきの水 墨うすく、ほのかにまぎらはして、

’あてやかに書かれたり’と見給ひて...” (“On a piece of paper colored pale purple of the front and blue on the back, written in thin, pale, barely legible ink was, 'By revealing your thoughts to people they appear shallow. If it is something you feel deep in your heart, you should keep it to yourself.' It was seen and remarked of, 'It is written sublimely." "), Sakakura (ed.): Yoru no Nezame op. cit. (note 9), p. 61.

12 “思ふことを人にいひ續けん言の葉は、もとよりだに、はかぐ しからぬ身を、まいて、なつかしく、ことわるべき人さへなけれ ば、た ゙硯にむかひて、思ひあまるをりには、手習いをのみ、たけ き事とは、書きつけ給ふ。” “I have never been of the constitution to reveal my inner feelings to people. What is more, I am not close enough to anyone to explain the circumstances, so whenever I am overcome with such thoughts, I spend that time in front of the inkstone, occupying myself 
$k a n j i$, also called mana) was the language used in official documents, it was customary to use kana for correspondence of a personal and social nature. The overall cultural focus was on kanji, so it is easy to see how $k a n a$, as a medium of expression based on people's true feelings, was all the more suited to conveying emotions.

The very word shōsoku (消息) evokes the essential elements of kana brush strokes. The first half of the compound, 消, means 'to die', while the second half, 息, means 'to live'. As a compound, shōsoku refers to people's movements and developments and their safety and health. The reason the word shosoku also refers to handwritten correspondence is that letters were one of the few means of letting people know you were safe and well. On a more fundamental level, shossoku is likely related to the movements that appear in a person's brush strokes, and in turn, a person's breathing.

Whenever flow or undulation is perceived in a brush stroke, there had to have been a rhythm to the series of brush movements made when writing that stroke. Based on this principle, the flow of brush strokes is present not only where they are visible as joining strokes, but also where they do not appear as actual brush strokes, i.e. where the brush slid over the paper without touching it. The eye perceives these uneven blank spaces that appear in some places between characters as full intervals.

\section{IV.2. KANA INTERVALS AND BRUSH STROKE CHARACTERISTICS: FROM THE PERSPECTIVE OF MORPHOMATA}

Kana brush strokes done with a calligraphy brush possess a wavelike rhythm. It is a rhythm generated by brush strokes which, combined with factors such as the amount of brush pressure and the speed of the strokes, move along the paper in three dimensions. These three-dimensional waves, which are the result of combined horizontal (i.e. parallel to the plane of the paper) and perpendicular motions, are reduced to shapes on a two-dimensional plane (i.e. the paper) when transferred to the paper by the brush. When we view the shapes that appear on paper in terms of waves, we see that differences in amplitude and velocity give rise to variations in character size, the density of spaces between characters, and the amount of ink in the brush, in addition to concord among different lines.

whole-heartedly by writing practice poems to take my mind off those things."); Tokuhei Yamagishi (ed.): Genji Monogatari ("The Tale of Genji"), Vol. 5, (Tenarai), Nihon Koten Bungaku Taikei (18). Iwanami Shoten 1963, S. 391-392. "Tenarai-no-maki" ("Practicing calligraphy scroll") provides frequent examples of this. 
While the simplified forms of kana are all asymmetrical with a sideways tilt, they possess the visual equivalent of musical phrases. What define the 'flow' of kana are these phrases, which, when coupled together, form the flow of lines. Kana cease being individual characters, instead taking on artistic significance as components of phrases and playing a major role in composing 'lines'.

These artistic sensibilities are similar to the concept of 'morphomata'. 'Morphomata', or 'morphome', is a neologism that derives from the Greek mórphoma. It has yet to appear in published dictionaries, but means something akin to 'fragment', but instead of the fragment being seen as part of a whole, it represents a fragment in the sense of a being a creative element that acts as a vehicle for forming whole perspectives. Kana brush strokes are manifestations of unique waves, and from these waves are formed 'phrases' that, in addition to playing an integral role in the shaping of curves, guide and direct the whole. These 'phrases' are meaningful units in that they intermediate between individual hiragana characters (the smallest whole unit of the kana syllabary) and poems or whole calligraphic works. The beauty of renmen (joined writing) emerges with the intervals that appear between these phrases.

A cultivated sensitivity to and mastery of such intervals is what leads to a heightened awareness of the true significance of renmen techniques (how to sever and connect strokes) in kana calligraphy, which is itself a heightened awareness of the true nature of characters. One of the ideals of calligraphy is to express temporal qualities with strokes of the brush and spatial qualities with the form of the calligraphic work, and doing so in a manner that results in an overall appearance that integrates both. This paper has referred to the surface of the paper upon which calligraphy is written not as a flat surface, but as a space, as its form is more implicative of the latter. When the blank spaces become more apparent, the intervals in the calligraphy come to surface as well. This facet is most clearly embodied in the chirashi-gaki style discussed above.

Nowadays, there are around 10 million people in Japan who practice calligraphy and truly engage with characters. The perspectives of production and appreciation that have been explored in this paper are being put into practice in modern-day calligraphy (although theoretical frameworks have yet to be established). It appears as though modern Western European views of art and Japanese views of art are blending together, and that modern calligraphy has been passed on in these two disparate worlds without contradiction. The wave of modernization that began to overtake Japan in the Meiji Era meant that the world of calligraphy now 
had in exhibitions a new forum for appreciation. The microcosm born of fine strokes and lines is expanding to include more giant characters and embarking down the road of mural art. One thing, however, that has survived to this day is the ongoing endeavor to explore through classical articles of calligraphy the possibilities of brushwork and artistic shapes.

\section{LIST OF ILLUSTRATIONS}

1 Tokyo National Museum.

2 The Gotoh Museum, Tokyo.

3 Tekisui Museum.

4 Kōsetsu Museum.

5 Tōyama Memorial Museum. 

MATHIAS OBERT

\section{CHINESISCHE SCHRIFT UND LEIBLICHES DENKEN}

\section{SCHRIFTGESTALT UND SPRACHVERHALTEN}

Dieser Beitrag stellt aus einer phänomenologischen Perspektive Überlegungen zur anschaulichen Gestalt der chinesischen Schrift vor. Ziel ist der Entwurf einer philosophisch bedeutsamen Alternative zu jenem Grundriß, den die gelehrte Beschäftigung mit China seit Leibniz vorgezeichnet hat. Angesichts der Fragwürdigkeit verbreiteter Lehrmeinungen geht es um eine neuartige Annäherung an die alte Streitfrage nach der Bildhaftigkeit jener Schrift. Quer zur Sprachwissenschaft soll ein anderer Blick auf deren Gestalt- und Ausdruckswerte eröffnet werden. Letztlich lässt sich auch auf diesem transkulturellen ,Umweg' das logozentrische Paradigma des alten Europa hinterfragen und eine sprachphilosophische Neuorientierung anbahnen. ${ }^{1}$ Allerdings ist es diesem Beitrag nicht um die chinesische Sprache im Ganzen zu tun, ${ }^{2}$ sondern um Aspekte der Schriftlichkeit in ihrer philosophischen Bedeutung für die Gegenwart.

1 Einen ähnlichen Ansatz verfolgt Rolf Elberfeld in Sprache und Sprachen. Eine philosophische Grundorientierung (Freiburg i. Br. und München 2012, S. 113-126 und 148-165).

2 Vgl. John DeFrancis: The Chinese Language, Fact and Fantasy. Honolulu 1984; Christoph Harbsmeier: Wilhelm von Humboldts Brief an Abel Rémusat und die philosophische Grammatik des Altchinesischen. Stuttgart-Bad Cannstatt 1979; Christoph Harbsmeier: Language and Logic in Traditional China. Cambridge 1998; Mathias Obert: Philosophische Sprache und hermeneutisches Sprechen: Kritische Überlegungen zur chinesischen Sprache und ihrer Beschreibung aus philosophischer Sicht. In: Zeitschrift der deutschen morgenländischen Gesellschaft 155 (2005), S. 545-575. 
In der führenden linguistischen Forschung zu China bleibt der für jede Schriftdeutung wichtige Vorgang der Sinnstiftung in der Anschauung systematisch ausgeblendet. Es wird vorausgesetzt, dass Schrift lediglich die gesprochene Rede sichtbar mache und auf dem Umweg über die Kundgabe intelligible Gegenstände anzeige. Ein platonisierender Theorierahmen verschließt von vornherein sinnträchtige Alternativen, wie sie der chinesische Schriftgebrauch eindringlich fordert. Insbesondere eine Neuerkundung dessen, was ein ,Bild' ausmacht, am Leitfaden der besonderen Bildhaftigkeit jener Schriftzeichen wurde bislang nicht als Forschungsaufgabe wahrgenommen.

Die Frage nach den Gestaltwerten der chinesischen Schrift führt auf die Verwurzelung der Sprachlichkeit im Weltbezug des leiblichen Selbst zurück. Vor allem Maurice Merleau-Ponty setzt entschieden bei der Rede als einem "gestischen Bedeuten“ (signification gestuelle) ${ }^{3}$ an und markiert damit eine Hinwendung der Sprachphilosophie zum Gesamtverhalten des Menschen. Auf diesem Wege können nicht nur oft übersehene Phänomene der Zeitlichkeit und der Bewegung im menschlichen Sprachverhalten Berücksichtigung finden, auch eine tiefere Verbindung zwischen Rede und Schrift tritt nun zutage. Das mentale „Wortbild“ (image verbale) wird nach Merleau-Ponty strukturiert durch „eine gewisse leibliche Haltung, eine spezifische Art und Weise dynamischer Spannung" (une certaine attitude corporelle, un mode spécifique de tension dynamique). Auch sei sprachlicher Ausdruck stets aus dem leiblichen Zusammenspiel der Sinne heraus zu verstehen; in unauföslicher Einheit stelle jeder Sprachausdruck zugleich etwas Gesagtes, Gehörtes und Angeschautes dar. ${ }^{4}$ Ausdrücklich betont Merleau-Ponty, dass das gesprochene wie das geschriebene Wort noch vor der Entfaltung seiner sprachlichen Bedeutsamkeit ursprünglich durch leibliche Zustände hindurch erschlossen werde. Dementsprechend vollziehe sich das Erlernen eines neuen Wortes als Nachahmung eines Stils, gleich der einer Geste. Ein unbekanntes Wort bilde zuallererst eine „Modulation meines Leibes als eines Seins-zur und in-der-Welt" (modulation de mon corps comme être au monde) aus. ${ }^{5}$

Merleau-Pontys Sprachauffassung mag befremden; gerade für ein Verständnis der chinesischen Schrift erweist sie sich jedoch als äuBerst hilfreich. Es gilt, diese Schrift als ein entscheidendes Moment im

3 Maurice Merleau-Ponty: Phénoménologie de la perception. Paris 1945, S. 208.

4 Ebd., S. 272 f.

5 Ebd., S. 461. 
praktischen Verhalten des Menschen zu seiner Umwelt in den Blick zu nehmen. Entgegen der kulturhistorischen Überzeugung von einer nachträglichen Verschriftung verlautbarter Rede muss aus phänomenologischer Sicht grundsätzlich auf die Sprachpraxis, das heißt auf die Gebrauchsweisen der Schrift innerhalb des Sprachverhaltens im ganzen, zurückgegangen werden. Weil jede Sprache eine organische Einheit darstellt, wirkt jede Schrift nach ihrer Einführung zurück auf das gesprochene Wort. Die Gestaltfülle des chinesisch Geschriebenen entfaltet ebenfalls in allen sprachlichen Vollzügen ihre affektive Bindungskraft, leistet ihrerseits eine Sinnstiftung.

Bis heute sind alle Versuche einer radikalen Phonetisierung oder gar Alphabetisierung des chinesischen Schriftsystems gescheitert. Ein Grund dafür liegt gewiss in dessen Offenheit für unterschiedliche Aussprachen und Verwendungsweisen von China über Korea und Japan bis nach Südostasien, ein weiterer darin, dass mit einer reinen Lautschrift die Fülle an Homophonen im buchstäblich einsilbigen chinesischen Wortschatz offenkundig nicht beherrschbar wäre. Der Hauptgrund freilich dürfte darin liegen, dass im Laufe der Geschichte das Geschriebene sich innerhalb des chinesisch Gesprochenen einen unverzichtbaren Platz erobert hat, dass die Schrift integraler Bestandteil jeder Äußerung geworden ist. Damit weisen alle sprachlichen Phänomene des Chinesischen eine prinzipielle Diskrepanz gegenüber dem Paradigma der sekundären Verschriftung auf.

Auch wenn die chinesische Schrift ein Stück weit unabhängig von der Rede funktioniert, weshalb sie oft als ,Bilderschrift' angesehen wurde, muss sie doch gerade umgekehrt als ein integrales Moment der Sprachlichkeit selbst begriffen werden. Wo vom Leser mitunter einzelne Schriftzeichen in einem Text verstanden, gleichwohl nicht laut gelesen werden können, da fehlt diesem nicht etwa die Aussprache; vielmehr zeigt sich darin der von Alphabetschriften abweichende Grundcharakter dieser Schrift. Schon vor der lautlichen Kundgabe legen die Schriftzeichen allein vermittels ihrer Anschauungsgestalt ein Verständnis nahe; infolgedessen ist die chinesische Sprachlichkeit insgesamt durchdrungen von gestalthafter Anschauung. Im Zuge einer einzigartigen Symbiose zwischen Schrift und Kundgabe wirken schriftliche Äußerungsformen tief in die Ausdrucksmöglichkeiten der Rede zurück. Anders als im Falle von Alphabetschriften wird die Sinnstiftung insgesamt weithin begleitet - wenn nicht getragen - von der Anschauung oder Vorstellung relevanter Schriftzeichen. Der volle semantische Gehalt der Rede erschließt sich letztlich erst im Hinblick auf Schriftkontexte, über das Imaginieren geschriebener Zeichen. Semantische Bezugnahme, Differenzierung und Präzisierung im Sprechen 
und Sprachhören werden maßgeblich gestützt durch die Schreibgestalt. Vermittels assoziativer Mitvergegenwärtigung ist jede Äußerung umgeben von bedeutungs- und erlebnismäßigen Kontexten, welche die Schriftgestalt als solche entfaltet. Diese gegenseitige Durchdringung von Schrift und Rede reicht bis ins Alltagsleben, beispielsweise in die ,Zeichenspiele ${ }^{6}$ der Warenwerbung hinein. Eine Betrachtung der chinesischen Sprache unabhängig von der Schrift ist daher prinzipiell verfehlt.

Die fraglose Übernahme der klassischen aristotelischen Lehre, wonach ein mit Buchstaben oder Silbenzeichen geschriebenes Wort für einen lautlichen Bedeutungsträger, dieser wieder für eine Idee oder Sache stehe, leitet die gesamte moderne Erforschung des Chinesischen, führt indes zu einer Verkennung der spezifischen Sachverhalte. Üblicherweise wird die Betrachtung der Schrift verengt auf eine Analyse des einzelnen Zeichens, denn im Rückgang auf dieses lässt sich der Wortschatz scheinbar schlüssig in selbständig bedeutsame Elemente gliedern: Ein Zeichen steht für ein Wort und eine Bedeutung. Doch entgegen dem aristotelischen Schema hält das Zeichen auch nicht annähernd eindeutig eine bestimmte Lautfolge fest. Infolge des gängigen Sprachverständnisses muss eine solche Schrift daher als einigermaßen unpraktikabel erscheinen; ihre bildhafte Besonderheit muss ihr zwangsläufig als Nachteil ausgelegt werden. Gegen diese Unterstellung spricht andererseits ihre kaum je angefochtene Verwendung im Rahmen höchst komplexer Lebens- und Kulturformen. Zudem scheint gerade in der verleugneten Bildhaftigkeit die bis in die moderne Computer-, Telekommunikations- und Netzsprache hinein nachweisbare Anziehungskraft und praktische Durchsetzungsfähigkeit des alten chinesischen Schriftsystems zu gründen. Es gilt daher, um nicht von der scheinbaren praktischen Untauglichkeit des einzelnen Schriftzeichens in die Irre geführt zu werden, mit Merleau-Ponty den Gesamtstil nicht bloß der Sprache, sondern auch der Schrift - verstanden als Gestaltganzheit eines gelebten Weltbezuges ${ }^{6}$ - in den Blick zu nehmen.

Die Erkundung der chinesischen Schrift sollte auch deshalb auf den Organismus der ganzen Schrift und den lebendigen Schriftgebrauch abzielen, weil der theoretische Primat des gesprochenen Wortes als Grundeinheit der Sprache im Falle des Chinesischen schwerlich einlösbar ist. Empirisch gesehen muss sich gerade die Sprachwissenschaft selbst - zumal in allen wegweisenden Untersuchungen zu den vormodernen Verhältnissen - noch vor den verlautbarten Ausdrücken immer

6 Ebd., S. 209. 
auf Geschriebenes beziehen. Die grundlegenden Urteile der Linguistik stützen sich in einem unkritischen quid pro quo paradoxerweise in erster Linie auf schriftlich angezeigte Elemente der Sprache, so gut wie niemals hingegen unmittelbar auf die verlautbarte Rede. Ohne die Schriftebene wäre die Fachwissenschaft aufgrund der wuchernden Homophonie und buchstäblichen Einsilbigkeit des Chinesischen kaum in der Lage, diskrete semantische Grundeinheiten zu isolieren und in Ableitungsverhältnissen zu verknüpfen.

Nicht von ungefähr schwankt bis heute die Übersetzung des europäischen Begriffs ,Wort' zwischen,Wort der Rede' ( $c i$ 詞) und ,Zeichenwort' ( $z i$ 字). Aus kulturhistorischer Sicht hat nicht die phonematische Bildung, sondern das ,geschriebene Zeichenwort' (wén zi 文字) als linguistisch elementar zu gelten. Ein ,Zeichenwort' ist freilich keine bloße Anzeige für einen Redeteil, ist kein ,Wortzeichen'. Zeichenwort' bedeutet gerade nicht ausschließlich das sichtbare Zeichen. In dem Begriff ,Zeichenwort' sind von vornherein die schriftliche und die lautliche Ebene als sich gegenseitig durchdringend zu denken. Die gesprochene Rede ist ihrerseits in ihrer Gesamtheit als gelebte Praxis integraler Bestandteil jedes Zeichenwortes. Dieses kennt eine, bisweilen mehrere Lesungen, und es besitzt jeweils bestimmte sprachliche Verwendungsmöglichkeiten und -kontexte. Darin unterscheidet es sich ebenso vom kontingenten ,Zeichen' anderer Schriftkulturen wie von ,Symbol' und ,Abbild' in der Kunst. Auch tut eine weit verbreitete Schriftunkundigkeit dem heuristischen Prinzip von der Gleichrangigkeit der Schrift keinen Abbruch, denn die historisch gewachsene chinesische Sprachlichkeit kann insgesamt nicht mehr auf den Gebrauch der Schriftzeichen verzichten - was zumal angesichts derjenigen komplexeren Äußerungsformen unmittelbar einleuchtet, die das öffentliche Leben, philosophisches Denken wie literarischen Ausdruck tragen.

Ausschlaggebend für die These von der theoretischen Bedeutsamkeit des Zeichenwortes für die Sprachphilosophie ist also erstens, dass das Zeichenwort in sprachpragmatischer Hinsicht durch die Geschichte hindurch maßgeblich an der Ausbildung des Sprechens beteiligt ist. Sodann kann das schriftliche Zeichen genau in dem Maße nie auf Lautelemente reduziert werden, in dem es in der Anschauung gestalthaft wirkt und seine eigenständige Sinnerfüllung gegenüber der Ebene einer rein lautlichen Sinnanzeige mit sich führt. Insofern die Schrift über die Anschauung und im bildhaften Vorstellen gegenüber der gesprochenen Rede eine eigene Bewegung inmitten des gesamten sprachlichen Verhaltens anstößt, geht sie nicht restlos in einem phonologisch-lexematisch erschlossenen Sprachsystem auf. 


\section{WAS IST EIN CHINESISCHES SCHRIFTZEICHEN?}

Weit verbreitet ist bis heute die Ansicht, chinesische Schriftzeichen seien Bildzeichnungen, ,Piktogramme'; ebenso ist selbst in Fachkreisen mitunter noch die Rede von ,Symbolen' und ,Ideogrammen'? Demnach stünde jedes Zeichen - unabhängig von der Aussprache - für eine bestimmte Sache oder ,Idee', etwa das Zeichen 人 (rén) ${ }^{8}$ für ,Mensch', 性 (xing) für ,Natur', 善 (shàn) für ,gut'. Der deutsche Satz, des Menschen Natur ist gut $^{`}$ könnte somit wie das klassische Zitat aus dem Buch Meng $Z i$ 孟子 chinesisch geschrieben werden: 人性善. Befestigt hat die Rede von der Ideographie sicherlich der Umstand, daß diese Schrift in unterschiedlichen Lautsystemen wie dem Pekinesischen einerseits und dem Kantonesischen andererseits ohne Abstriche zum Einsatz gelangt, auch dass sie selbst in dem fremden Sprachgefüge des Japanischen, losgelöst von der ursprünglichen Aussprache und Grammatik, noch leidlich ,funktioniert'.

Der Begriff der ,Ideographie‘ ist indes überholt, weil er allzu sehr vereinfacht und überdies auf einer idealisierenden Unkenntnis der chinesischen Sprachstruktur und des Schriftgebrauchs beruht. Gegen diese populäre Auffassung hat die neuere Forschung in der Konzentration auf eine phonetische Rekonstruktion der chinesischen Schriftentwicklung eine Reihe von ebenso ernüchternden wie berechtigten Erkenntnissen gewonnen. ${ }^{9}$ In der euro-amerikanischen wie sogar in der chinesischen Linguistik hat sich daher mittlerweile die gegenteilige Doktrin durchgesetzt, die chinesischen Schriftzeichen seien durchweg nach dem aristotelischen Muster einer Aufzeichnung von phonematischen Redeteilen zu verstehen. Als namhafter Vertreter dieser Forschungsmeinung hält es beispielsweise William Boltz kategorisch für unnötig, die Alternative

7 Vgl. Bernhard Karlgren: Sound and Symbol in Chinese. London 1923. 8 Wo im folgenden ausdrücklich auf die Gestalt der Zeichenwörter abgehoben wird, ist entgegen den Gepflogenheiten die Lesung in Umschrift in Klammern gesetzt.

9 Peter A. Boodberg: Some Proleptical Remarks on the Evolution of Archaic Chinese. In: Harvard Journal of Asiatic Studies 2 (1937), S. 329-372; P.A. Boodberg: ,Ideography' or Iconolatry? In: T’oung Pao 35 (1940), S. 266-288; Ulrich Unger: Aspekte der Schrifterfindung: Das Beispiel China. In: ders.: Kleine Schriften. Hrsg. von Hans Stumpfeldt und Martin Hanke. Gossenberg 2009, S. 31-52. 
zwischen ,Bilderschrift" und ,Lautschrift' weiter auszudifferenzieren. ${ }^{10} \mathrm{Als}$ Hauptargument dient ihm, daß ein Großteil der Schriftzeichen seine Bedeutung tatsächlich einem Verfahren lautlicher ,Entlehnung' (jiă jiè 假借) verdankt. Es handele sich daher weder um begriffsanzeigende ,Ideogramme' noch um sachanzeigende ,Piktogramme', vielmehr um wortanzeigende ,Logogramme': Mit einem Schriftzeichen werde eine diskrete sprachliche Äußerungs- und Sinneinheit festgehalten. ${ }^{11}$

Nun steht außer Zweifel, dass das chinesische Schriftsystem sich schon im frühen Altertum von ,piktographischen' Ursprüngen gelöst und hauptsächlich nach einem phonetischen Schematismus fortentwickelt hat. ${ }^{12}$ Das Geschriebene darf daher sicherlich nicht als Abbild der sichtbaren Welt verstanden werden. Andererseits kann es indes ebensowenig restlos auf die Aufzeichnung mündlicher Verlautbarungen, das Sichtbarmachen von Gehörtem reduziert werden. Offenkundig operieren die chinesischen Schriftzeichen irgendwo zwischen diesen beiden Extremen - und von Fall zu Fall an einer anderen Stelle in diesem Feld.

Als Argument für die ,Logographie' kann gelten, dass in einer Reihe von Fällen einzelne Schriftzeichen zwar bis zu einem gewissen Grade semantischer Allgemeinheit losgelöst von einer bestimmten phonematischen Entsprechung als bedeutungtragende Anzeiger für Dinge, Sachverhalte oder abstrakte Vorstellungen fungieren können, daß diese bildähnliche funktionale Übertragbarkeit von Schriftzeichen jenseits einer natürlichen Sprache gleichwohl prinzipiell stets einen sozio-linguistischen Äußerungshorizont voraussetzt. Der Sinngehalt der allermeisten Zeichenwörter ergibt sich auch gewiss nicht in reiner Anschauung, sondern nur durch den Bezug auf ein bestimmtes Sprachgefüge und dessen geschichtlichen Gebrauch. Im Kampf der Linguistik gegen Vorurteile zur Bildhaftigkeit der chinesischen Schriftzeichen sind diese Überlegungen zweifellos berechtigt; sie vereinfachen indes über Gebühr und verdecken so, was eigentlich in Frage steht. Bis heute haftet selbst nach Boltz der $A n-$ schauungsgestalt einzelner Schriftzeichen noch das Erbe piktographischer Ursprünge, eine nicht restlos aufgelöste ,Semantizität' an..$^{13}$ Diese gleichsam bildliche Sinnstiftung tritt sicherlich in den meisten Fällen hinter

10 Vgl. William Boltz: The Origin and Early Development of the Chinese Writing System. New Haven 1994, S. 3-6 bzw. 16 f.

11 Vgl. ebd., S. 5-7 bzw. 31.

12 Vgl. Bernhard Karlgren: Grammata Serica Recensa. Stockholm 1957; Boltz: Chinese Writing System (wie Anm. 10), S. 90-125.

13 Vgl. Boltz: Chinese Writing System (wie Anm. 10), S. 13 bzw. 168-172. 
die komplexere Bedeutsamkeit auf der Lautebene zurück. Allerdings entfaltet diese Bildkraft im Hintergrund des Leseverständnisses ihre eigene Wirksamkeit; sie sollte daher nicht lediglich als Makel eines im Prinzip längst von der Bildhaftigkeit befreiten Schriftsystems betrachtet werden. Dieser, anschauliche Überschuss' in der Semantik der Zeichenwörter ist gerade das Problem. ${ }^{14}$ Angesichts eines über zweieinhalb Jahrtausende fortgesetzten schöpferischen und wirkungsvollen Umganges mit eben diesem vermeintlichen Gestaltrest kann das Phänomen nicht mit dem Hinweis auf eine, unvollendete' historische Entwicklung abgetan werden.

Darüber hinaus bleibt im Begriff des ,Logogramms' die Frage, was nun wiederum ein, Wort' sei und wie ein Wort sich zum Gesamtgefüge der Sprache und zum Denken verhalte, gänzlich ungestellt. Gerade im Hinblick auf das Zusammenspiel von Verlautbarung und Zeichenwort in der ostasiatischen Kulturgeschichte ist dies indes ganz und gar kein triviales Problem. Noch augenfälliger als im Chinesischen tritt diese Thematik im Japanischen ans Licht. In der parallelen, teils sogar alternierenden Verwendung von chinesischen Schriftzeichen und japanisch-phonetischen Silbenzeichen in ein und demselben Kontext tritt die Verschränkung von Zeichen- und Lautwort unmittelbar zutage; zugleich wird so die von der Linguistik unterschlagene Kluft greifbar, die zwischen einem Wortzeichen und einem chinesischen Zeichenwort besteht. Im japanischen Umgang mit der chinesischen Schrift tritt die theoretische Diskrepanz zwischen einer sichtbaren, kodifizierten Bezeichnung für ein Phonem oder ein Wort der verlautbarten Rede einerseits und einem nur als sichtbare Gestalt sinnerfüllten Zeichenwort unmißverständlich zutage. Beispielsweise kann für das ,Ding/Geschöpf' mal das chinesische Zeichenwort 物 - japanisch mono gelesen -, mal die Silbenschriftform $\circlearrowright$ (mono) Verwendung finden. Während nun auch eine Person ein ,Geschöpf' (mono) ist, sperrt sie sich gegen die Schreibung mit dem Radikal für, Vieh ‘ 牛 beziehungsweise 牛. Dasselbe Wort ,Geschöpf' erhält deshalb im Falle des Menschen die Schreibung 者 (mono), welche keine falsche Bildvorstellung weckt. Die gebräuchliche Anrede あなた (anata) für ,du/Sie‘ kann je nach Kontext - bei einheitlicher Lesung - mal mit den Zeichen 彼方 beziehungsweise respektvoll 貴方für, werter Herr' oder 貴女 für ,werte Dame', mal wieder mit 貴夫, ,mein Gatte', ausgeschrieben werden. Wo auf der Lautebene

14 Eine beachtenswerte Perspektive gegen die allgemeine Forschungsrichtung bietet Tze-wan Kwan: Abstract Concept Formation in Archaic Chinese Script Forms: Some Humboldtian Perspectives. In: Philosophy East \& West 61, no. 3 (2011), S. 409-452. 
der situative Bezugssinn unterschlagen wird, wirkt die Schreibung mit chinesischen Zeichen auf Anhieb differenzierend und klärend - und zwar unausweichlich schon in der Anschauung. Ein ,Elephant' kann entweder als Zeichenwort 象 oder in phonetischer Silbenschrift als ぞう $(z \bar{o})$ erscheinen - wenn nicht sogar die ,modernistische' Lesung als europäisches Fremdwort エレファント (erefanto) bevorzugt wird. Die Frage ist also: Bezeichnet tatsächlich das chinesische 象 wie ein bildlicher Anzeiger jene Sache, die ganz ebensogut mit ぞう,エレファント oder ,Elephant ${ }^{\star}$ bezeichnet wäre? Verweist es als ,Logogramm' auf ein chinesisches oder sino-japanisches Wort? Oder besteht nicht doch eine offenkundige Diskrepanz zwischen dem mit Gestaltcharakteren aufgeladenen Zeichenwort 象, der japanischen Wortsilbe ぞう, dem Fremdwort エレファント und dem Wortsinn ,Elephant'?

Aufgrund einer Vernachlässigung solcher gängigen Erfahrungen im Umgang mit der Schrift wird seitens der Sprachwissenschaft die Frage nach den funktionalen Grundelementen der Sprache immer schon in europäischer Weise vorentschieden. Im Zuge dessen wird die geschichtliche Komplexität lebendiger ,Sprachspiele ${ }^{\text {` }}$ die immer auch ,Zeichenspiele sind - unterschlagen und die Fülle der Zeichengestalt reduziert auf eine mentale Sinneinheit beziehungsweise auf ein Lexem. Eine eurozentrische Logik des Abgrenzens und Definierens verstellt da systematisch den Blick auf anschauliche Zusammenhänge wie nicht minder auf zeitlichereignishafte Vollzüge der Sinnstiftung. Übersehen wird die Struktur der Zeichengestalt als solche mit ihrer Binnengliederung und ihren vielschichtigen wahrnehmungs-, empfindungs- und bewegungsmäßigen Wertigkeiten. Ebenso wenig Beachtung findet der Assoziationshorizont aller Zeichen im Schriftsystem als einem regelhaft gebildeten Gestaltgefüge, das Ganze eines Textes oder der für die chinesischen Schriftzeichen entscheidende Akt des Schreibens. Ausgehend von der Streitfrage zwischen ,Piktographie', ,Ideographie' und ,Logographie' kann gleichwohl die bildhafte Eigentümlichkeit der chinesischen Schrift erhellt werden, sofern eine kritische Epoché hinsichtlich der eigenen Vorannahmen die Beobachtungen anleitet.

Zunächst einmal verdanken gerade die gebräuchlichsten Zeichenwörter ihren Ursprung tatsächlich einem Verfahren der Bildgebung, und bis heute haben sie eine unmittelbar bildhafte Ausdruckskraft bewahrt. Beispiele dafür sind zunächst alle ,sachanzeigenden' ( $z h \grave{\imath}$ shì 指事) Zeichen, etwa die unmittelbar eingängigen Zahlzeichen - (y) $)$, 二 (èr), 三 $(s \bar{a} n)$, ebenso die Zeichenwörter 上 (shàng) und 下 (xià) für, oben' und ,unten'. Vor allem aber gehören zu dieser Gruppe alle Grundwörter, die in 
der Frühzeit durchaus in piktographischer Manier ,dem Aussehen von Gegenständen ihre Zeichengestalt abschauten' (xiàng xíng 象形). Stellvertretend für gut zwei Dutzend tauglicher Beispiele betrachte man die ursprünglich runde, Sonne' 日 (ri), den teilweise abgeschatteten ,Mond 月 (yuè), den aufragenden ,Berg' 山 (shān), den astreichen, Baum' 木 $(m \grave{u})$, den geöffneten ,Mund' 口 (kǒu), den ,Menschen' auf zwei Beinen 人 (rén) oder die breithüftige, Frau'女 $(n \ddot{u})$. Bis in die Druckschrift hinein entfalten diese Zeichengestalten zumindest eine Ahnung von ihrer bildlichen Herkunft, und bildhaft unmittelbar verweisen sie schon in der Anschauung - also unabhängig von und vor lautlichen Verbindungen auf Sachen.

Weithin unbeachtet bleibt sodann dieser überaus wichtige Umstand: Die allermeisten Schriftzeichen sind aus einem oder mehreren Bestandteilen zusammengesetzt. Diese werden nach zwei Kategorien in lautanzeigende Komponenten und generische Denominativa gegliedert. Auf diese Weise sind insbesondere bildhafte Grundzeichenwörter wie die angeführten als eines oder mehrere Versatzstücke in eine beträchtliche Anzahl von Schriftzeichen integriert. Zumal aber immer dann, wenn diese bildhaft prägnanten Denominativa in einem zusammengesetzten Zeichen an markanter Stelle als eines der 214 ,Radikalzeichen' (bùshòu 部首) fungieren, gewährleisten sie im Leseakt unweigerlich eine anschauungsmäßige Vorstrukturierung der Sinnerwartung. Ein ,Brett' 板 (băn) ist offensichtlich ,aus Holz' (木), essen` 吃 (chī) tun wir mit dem, Mund (口), ,Mitgift‘ 妝 (zhuāng) gehört der, weiblichen` (女) Sphäre an. Die Radikale tragen nicht nur maßgeblich zur semantischen Ausdifferenzierung des Zeichenwortschatzes bei; während der Lektüre spielen diese rekurrierenden Gestaltelemente gerade aufgrund ihrer unmittelbar hervorstechenden Eingängigkeit eine entscheidende Auffassungshilfe. Aus diesem Grund ist es durchaus nicht übertrieben, in so gut wie allen chinesischen Schriftzeichen ein unmittelbar in der Anschauung entfaltetes Sinn- und Wirkungsmoment hervorzukehren. Selbstverständlich darf diese Beobachtung nicht mit der unhaltbaren Behauptung verwechselt werden, es handele sich bei jedem Zeichenwort im ganzen um ein ,Piktogramm'.

Gegen solche Überlegungen wird nun oft eingewendet, die denominativen Zeichenelemente hätten im Zuge des Abstraktionsprozesses fortschreitender Schriftbildung ihren bildlichen Ursprung hinter sich gelassen, was sie ursprünglich bezeichnet hätten, sei längst nicht mehr kenntlich. Doch die entscheidende Frage lautet nicht, ob die besagten Zeichenbestandteile dem Leser automatisch durch Ähnlichkeit bestimmte Dinge sichtbar machen - wie dies Piktogramme dem Prinzip nach leisten. 
Ein der chinesischen Sprache und Schrift Unkundiger vermag keinesfalls aus generischen Denominativa die richtigen Rückschlüsse auf die Bedeutung zusammengesetzter Zeichen anzustellen. Die Schrift muss sicherlich ,gelesen', das heißt mit sprachlich vermitteltem Sinn erfüllt werden, sie will nicht lediglich wie ein Bildsymbol betrachtet sein. Gleichgültig für die Frage nach der prinzipiell bildhaften Wirksamkeit der Schrift ist ebenfalls, ob generische Gestaltelemente stets auf die jeweilige Wortbedeutung hinführen oder mitunter eher davon ablenken. Zur Frage steht hier allein die anschaunngsmäßige Wirksamkeit der Zeichengestalt. Diese fußt auf einer Ausfächerung der Assoziationsfelder, die schon mit den bildhaft eingängigen Denominativa in rund 200 verschiedene Sachrichtungen weist. Aus vielfältigen Gestaltmomenten entspringt unmittelbar und mit Notwendigkeit ein feinsinniges Spiel mit unterschiedlichen Vorstellungshorizonten. Allein in diesem nicht-europäischen Untersuchungshorizont können assoziative Schichten der Schrift als leibliches Motiv und Movens gehoben werden. Insgesamt bleibt die Sprachwissenschaft dagegen in ihrem Feldzug gegen die Bildhaftigkeit zu Unrecht auf Probleme der Zeichenbildung und -ableitung fixiert.

\section{GESTALTHAFTE BEDEUTUNGSASSOZIATION}

Insbesondere wo Denominativa als ,Radikale in zusammengesetzten Zeichen auftreten, lenken sie infolge ihrer Häufigkeit und gestalthaften Prägnanz die Wahrnehmung auf Anhieb auf bestimmte Bedeutungsfelder hin; sie sortieren das Gelesene schon in der Anschauung nach bestimmten Hinsichten. Mit der Lautung haben diese markanten Zeichenbestandteile meist nichts zu tun. Doch diesen Schritt hat der Lesende unwillkürlich immer schon vollzogen, noch bevor er die wesentlich unschärferen phonematischen Indikatoren an einem Schriftzeichen wahrgenommen, es über eine erlernte Lesung als einen bestimmten Redeteil erkannt hat. Noch ehe also im Akt des Verstehens dem Schriftzeichen seine Bedeutung im Text entnommen wird, ist sein Verständnis bereits in sinnlich erfüllter Weise vorgeprägt von hervorstechenden Gestaltmomenten.

Ein paar schlichte Beispiele können dies veranschaulichen. Gerade weil Wasser sichtlich trüb und verschmutzt sein kann, darum ist auch

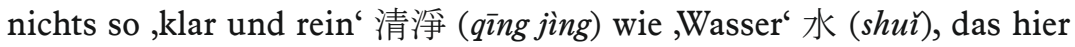
als Radikal die Gestalt von drei Tropfen \& angenommen hat. Zweifellos veranschaulichen diese Tropfen nicht die Idee der Reinheit; dazu sind sie viel zu unpräzise. Zudem finden sie sich in rund 1800 weiteren 
Schriftzeichen mit ganz anderen Bedeutungen ebenso wieder. Allerdings leiten diese Tropfen die Assoziation des Lesenden unweigerlich auf sinnliche Vorstellungen von Wasser. Noch bevor er ,klar und rein' liest, schaut er bereits in der Zeichengestalt Flüssiges an - er sieht Transparenz, spürt kühles Naß auf der Haut, riecht ein Gewässer, vernimmt das Sprudeln einer Quelle. Im assoziativen Zusammenwirken aller Sinne erhält der Lesende durch die Anschauung schon eine leibhaftig empfundene Ahnung von der Bedeutungsrichtung, noch ehe die semantische Klärung im Redekontext stattfindet.

Im Zeichen 味 (wei) springt der geöffnete, Mund' 口 (kǒu) ins Auge. Noch bevor dieses Zeichenwort vom Lesenden als ,schmecken' verstanden ist, hat er diese Bedeutung in der Anschauung leibhaftig mit dem eigenen Mund vorgeformt. Nicht die Idee des Geschmackssinnes oder die Erinnerung an Akte des Schmeckens, der Mund des Lesenden ist es, der in der Anschauung bereits affektiv angerührt und als sinngebend aufgerufen wird. Die zweigliedrige Wendung 腐爛 (fǔ làn) für, verwest/ verdorben' hinwiederum mag schon darum unmittelbar ekelerregend aussehen, weil der Lesende auf den ersten Blick das ,Fleisch' 肉 (ròu) wahrnimmt, welches sich bei Hitze zersetzt - wie beim zweiten Zeichen im Radikal ,Feuer'火 (hио̆) angedeutet. Die Vorstellung von Fleisch in der Hitze kann gewiß sowohl positiv als auch negativ besetzt sein. Demnach könnte auch ein nach Erhitzung zum Verzehr geeignetes Stück Fleisch in der Vorstellung auftauchen. Der Sprach- und Schriftkundige wird allerdings auf einen Blick eine Differenzierung zu einem Ausdruck wie 煮熟 (zhǔ shóu) für, gargekocht' vollziehen; in den vier kursiv ausgeschriebenen Punkten … für dasselbe Radikal ,Feuer 火 火 (huŏ) häuft sich sichtlich die Hitze, hier indes zu einem guten Zweck. Zweifellos erst im Zusammenhang mit dem ganzen System von Schrift und Sprache kann also der Ausdruck 腐爛 seine abstoßende Färbung erhalten. Diese aber kann unter Umständen infolge der mehr oder weniger bildhaft ausstrahlenden Zusammenspiels der Radikale ,Fleisch“肉 und ,Feuer ${ }^{\text {火 } 火 ~}$ geradezu leibhaftig erfahren werden. Die entsprechenden Assoziationen werden hier nicht ausschließlich lautlich induziert, sie tauchen schon vor der vollständigen semantischen Erschließung in der Vorstellung auf. Wo die Einnahme verdorbener Speisen im eigenen Leibsein Spuren hinterlassen hat, kann ein leiblich empfundenes Abgestoßensein schon in der Anschauung den Wortsinn aufrufen, sowie der Blick die beiden Radikale als einen bildhaften Sinnbezug erfasst.

Dass Lautassoziationen in der Sprachgeschichte zu einer feineren Bedeutungsdifferenzierung führen, steht außer Frage. Insbesondere 
dichterische Wendungen ziehen indes erheblichen Vorteil aus jenem vermeintlichen, Überschuss an Gestalt', um den es hier zu tun ist. Diese gestalthafte Bedeutsamkeit strahlt nicht nur vom Radikal auf das ganze Zeichen aus, sondern ebenso auf längere Ausdrücke, letztlich den ganzen Satz. Schlechtwetter beispielsweise kann geschildert werden als ,wirbelnder Wind, plötzlicher Regen' 蝡風驟雨 (piāo fēng zòu yūu). Sogleich ersehen wir das besondere, Wirbeln' des Windes im Radikalzeichen,Wind“ 風 (fēng), weil nur der Wind so unstet, dahintreibt‘ 飄 (piāo). Was im Wasser ,forttreibt' 漂 ( $p i \bar{a} o$ ), treibt in der Tat auf andere Weise, wird daher mit

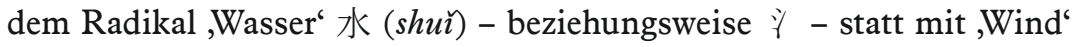
風 geschrieben. Linguistisch dasselbe Lautwort piāo wird somit erst als Zeichenwort semantisch prägnant. Die unterschiedene Schreibung markiert indes nicht nur einen Bedeutungsunterschied; durch die Häufung des,Windes' (風) wird der Blick des Lesenden mit einem Schlag auf diejenige Gestalt gelenkt, welche das geschilderte Wetterphänomen in der leibhaftigen Erfahrung verkörpert - die Unrast des Windes. Das ,plötzliche 驟 (zòu) Aufbrausen des, Regens'雨 (yũ) steht dem an evokativer Kraft nicht nach. Im Radikal ,Pferd‘ 馬 (mă) tost der Regen so heftig und ungestüm wie wilde Pferde. Das Phänomen des Sturmregens findet über die Wahl des Radikals seine sinnliche Verkörperung in der Zeichengestalt, und an dieser Stelle wird es beim Lesen vorab schon in der leiblichen Bewegungserfahrung vom Ungestüm eines Pferdes erlebt.

Wohlgemerkt steht in diesen Beobachtungen nicht zur Frage, ob das Radikalzeichen 馬 überhaupt erkennbar aussieht wie ein Pferd. Nicht auf die alte Frage der Piktographie zielt das Argument, sondern auf eine Phänomenologie des Lesevorganges. Weil der Schriftkundige vertraut ist mit dem Zeichenwort 馬 für,Pferd', darum sieht er in der angeführten Wendung auf einen Schlag ein Pferd sich aufbäumen, wo Regen braust. Die Vorstellung von der Gestalt eines ungezähmten Pferdes stellt sich unmittelbar im Blick auf die Gestaltkomponente 馬 ein, ohne daß das ganze Zeichenwort 驟 (zòu) zuvor laut ausgesprochen und semantisch erschlossen sein müßte. Natürlich soll hiermit wiederum nicht behauptet werden, daß die ganze Vierzeichenwendung nicht auch auf der Ebene des gesprochenen Wortes ihre eigenen Ausdruckswerte entfalte. Herausgestellt wird lediglich, daß bereits auf der Ebene der Anschauung ausgehend von der Schriftgestalt eine eigene Assoziationskette ausgelöst wird. Auch vermag sich der Lesende dieser bildhaften Strahlkraft der Zeichen nicht zu entziehen, sie ergreift sein Denken unmittelbar, leibhaftig und mit Notwendigkeit. Selbst wenn er gemäß der Lautgleichheit im ersten Ausdruck ein ,Fortspülen im Wasser - also 漂 (piāo) - assozieren wollte, wird ihm 
dies schwerlich gelingen: Der,Wind“風 in dem Zeichenwort 飄 (piāo) wird diese Assoziation mit sinnlicher Gerwalt in den Hintergrund drängen.

Naturgemäß sind die Vorzüge dieser eigentümlichen Gestaltfülle auch mit Schwächen verbunden. Doch gerade das Phänomen der Fehlassoziation ist dazu angetan, das bisher Festgestellte ex negativo zu bestätigen. So unterscheiden sich etliche Schriftzeichen untereinander nur durch winzigste Gestaltvariationen, so dass oft erst Kontext und Sinnhorizont eine angemessene Differenzierung im Lesevorgang gewährleisten. Die ,Kauri-Muschel' 貝 (bei) ist noch recht leicht von, sehen'見 (jiàn) zu unterscheiden. Auch die homophonen Zeichenwörter für, echt' 真 $(z h \bar{e} n)$ und ,keusch'貞 $(z h \bar{e} n)$ dürften noch kenntliche Unterschiede im Aussehen aufweisen. Anfällig für Fehlschreibungen ist schon eher eine Gestaltähnlichkeit wie die von,vermeiden“免 (miăn) und,Hase“兔 (tù). Und sind die Zeichenwörter 戒戎戌戍戊 -, warnen' (jiè 戒), Waffe' (róng 戎), ,zwölfter Erdast/zwölf' ( $x \bar{u}$ 戌), Grenzgarnison' (shù 戍), fünfter Himmelsstamm / fünf' (wù 戊) - wirklich ohne Mühe zu unterscheiden? Und wie sieht es mit der Reihe 胃冒䡒 für ,Magen“ (wei 胃), ,Nachfahre“ (zhòu 胄) und ,Helm‘ (zhòu 贵) aus? Dabei steht die Komponente 月 mal verkürzt für ,Fleisch“肉 (ròu), mal für den, Mond‘ 月 (yùe).

Dass in solchen Fällen Verschreibungen und Fehlleistungen im Lesen auftreten können, liegt auf der Hand. Dass dies in pinselschriftlichen Stiltypen teils noch wahrscheinlicher ist als im Druck, teils hingegen aufgrund zusätzlicher Bewegungs- und Richtungsindizien in der Schreibgestalt eher ausgeschlossen werden kann, spielt hier keine entscheidende Rolle. Zu fragen ist, ob dergleichen Distinktionen auf der Anschauungsebene tatsächlich genauso wenig Anstrengung erfordern wie etwa die von ,Leib' und ,Laib' im Rahmen einer Lautschrift, oder ob die Eigenart der chinesischen Schriftzeichen nicht doch eine besondere Leistung des Anschauungsvermögens fordert - und fördert. Wenn ganz regulär schon kleinste Variationen in der angeschauten Gestalt aus einem Zeichenwort ein anderes machen, eine Bedeutung in eine ganz andere verwandeln, läßt sich daraus dann nicht folgern, dass diese Schrift dem Lesenden nicht bloß eine kontextabhängige Wahrnehmung oder die ,blinde' Beherrschung einer unübersehbaren Anzahl von - letztlich bis zu 50000 - Gestaltmustern abverlangt, sondern eine andersartige Fertigkeit? Diese besondere Fertigkeit wird in eurozentrischen Forschungsansätzen unterschlagen; sie beruht in einem physiognomischen Blick auf die Schrift.

Zum Verständnis eines chinesischen Textes reicht es nicht, dass während des Lesevorganges nach festen Regeln angeordnete Gestaltganzheiten korrekt wahrgenommen und jeweils einem diskreten Laut- und 
Sinngehalt zugeordnet werden. Um flüssig und erfolgreich zu verstehen, bedarf es einer intuitiven Erfassung feiner Ausdruckswerte. Schon auf der Anschauungsebene kommen neben einer primären semantischen Assoziationsarbeit unweigerlich sekundäre affektive Aspekte ins Spiel. Entgegen dem linguistischen Vorurteil wird hier nicht durch sukzessives Lesen analytisch entschieden, ob es sich um 兔 oder 免, um 戎 oder 戊 handelt, ob da die verlautbarten Wörter tù oder miăn, róng oder $x \bar{u} \mathrm{zu}$ lesen sind. Wo aus dem Kontext heraus die Erwartung auf einen ,Hasen oder ,vermeiden', ,Waffe' oder ,zwölf' geht, trifft die Anschauung eines Zeichens auf solche Sinnhorizonte von vornherein vermittels einer physiognomischen Aufmerksamkeit. Über sinnlich gestalthafte Assoziation wird je schon Relevantes von Irrelevantem geschieden; wie ein Hof umgibt eine Textpassage beim Lesen ein Feld von Gestaltvarianten. Innerhalb dieser Zeichenserie kann die tatsächlich gegebene Zeichengestalt gleich einem Affekt das Vorstellen unmittelbar anrühren und so das Vorverständnis erfüllen. Assoziiert wird also die jeweils bezeichnete Sache nicht unmittelbar, sondern über den Umweg der Zeichengestalt. Ein Zeichen ruft in der lebhaften Vorstellung appräsentierend andere Zeichen auf, und erst in einer zweiten Appräsentationsreihe tauchen Sachvorstellungen mit auf. Genau in diesem mehrschichtigen Lesevorgang liegt der Unterschied zur reinen Bildlichkeit und zur ,Piktographie‘

Im Absehen auf bestimmte Gestaltcharaktere neigt die ,Volksetymologie' dazu, einen inkorrekten Zusammenhang zwischen sprachgeschichtlich nicht verwandten Zeichenwörtern herzustellen. Auch die geschichtliche Konventionalisierung im literarischen Gebrauch der Schriftzeichen gehorcht nicht unbedingt phonetischen Gesichtspunkten, einer sachgemäßen Logik oder historischer Korrektheit. So ist ein Kranker gemäß dem Ausdruck jí bing 疾病 für ,Krankheit' dranghaft erregt, wie das erste Zeichenwort 疾 jí anzeigt. Dass dies keine freudige Erregung ist, vielmehr eine leidvolle, das signalisiert die Schreibung mit dem Radikal 广 (chuáng) - entweder für einen Menschen, der sich aus Schwäche auf einen ,Bambusstab“ (qiáng 鸟) stützt, oder für das ,Krankenlager (chuáng 升 oder 牀). ${ }^{15}$ Das ganz anders geschriebene, in der heutigen

15 Siehe zu diesem und allen weiteren Belegbeispielen die diesbezüglichen Einträge in: Li Fuzhen 李福臻 (Hg.): Zhongwen da cidian 中文大辭典, 10 Bände. Taibei 1985; Luo Zhufeng 羅竹風 u. a. (Hg.): Hanyu da cidian 漢語大詞典, 12 Bände. Shanghai 1993; Xu Shen 許慎: Shuo wen jie zi 說文解字. Taibei 1998; Wang Li 王力: Tongyuan zidian 同源字典. Beijing 2002; Ulrich Unger: Glossar des klassischen Chinesisch. Wiesbaden 1999. 
Standardsprache gleichlautende Zeichenwort 急 $(j i)$ bedeutet ebenfalls ,Erregung/Eile;, weshalb es in manchen Redewendungen austauschbar mit dem Zeichenwort 疾 gebraucht wird. Als Ausdruck für die leiblich bedingte ,Erregung' des Kranken wäre es gleichwohl untauglich, denn es enthält das Radikal ,Herz' 心 $(x \bar{i} n)$. Die ,Krankheit ${ }^{6}$ 疾病 (jí bing) kann trotz ähnlicher Wortbedeutung nicht mit dem falschen Zeichenwort für ,Erregung' als 急病 geschrieben werden; dem widerstrebt die generische Komponente 心 für den, inneren Sinn'. Umgekehrt wäre die innere Unrast, der ,Aufruhr im Gemüt', als 心疾 ( $x \bar{\imath} n j i)$ ebenso falsch geschrieben. Allein das Radikal ,Herz ‘ ist dazu angetan, den Lesenden unmittelbar in der Anschauung jene besondere, innere Erregung' durchleben zu lassen, die die Wendung 心急 ( $x \bar{i} n j i)$ ausdrückt. Die ,drängende Eile‘ eines Pferdes schließlich, welches, rasend schnell galoppiert' (jí chí 疾馳), kann durchaus mit jenem Zeichenwort für ,Eile‘ und ,Krankheit' 疾 geschrieben werden. Denn erstens hat das ,Pferd' 馬 (mă) in dem Zeichenwort 馳 (chî) für ,galoppieren` wie der Kranke einen Leib; nicht das ,Herz ‘ $(x \bar{i} n)$ galoppiert hier. Zudem scheint nun in der Anschauung die in der Gestalt des Radikals 店 liegende,Krankheit' an dem Zeichenwort 疾 (ji) für ,Erregung' anschaulich neutralisiert zu werden durch den anderen Bestandteil in demselben Zeichen, durch den ,Pfeil '矢 $(s h \grave{\imath})$ : Ein Pferd galoppiert gemäß dieser Schreibung eben nicht ,krankhaft erregt', es galoppiert sichtlich ,pfeilschnell'.

Auf der anderen Seite haben sich im gewohnheitsmäßigen Umgang mit den Schriftzeichen durchaus, Fehlschreibungen' etabliert. Auch dieses Phänomen lässt sich im Hinblick auf die Gestaltassoziation nachvollziehen. Beispielsweise bedeutet das Zeichenwort 癖 ( $p \grave{\imath}$ ) ursprünglich eine ,einseitig' unausgeglichene Verdauungstätigkeit infolge spätabendlicher Nahrungsaufnahme. Doch warum werden die zusammengesetzten Ausdrücke für ,Vorliebe‘ 癖好 ( $p \grave{\imath}$ hào) und ,Sucht‘ 癖嗜 ( $p \grave{\imath}$ shì) wegen des

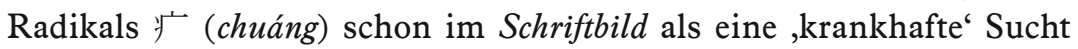
gebrandmarkt? Wird jede Art von, Vorliebe' als ebenso schädlich betrachtet wie Magengrimmen infolge unvernünftiger Essgewohnheiten? Wäre hier nicht sachlich gesehen eine andere Schreibung für die "Einseitigkeit“ einer Vorliebe angebrachter - etwa als 僻 (pi) mit dem Radikal ,Mensch 人 (rén) - beziehungsweise $\uparrow$ - an der Seite? Semantisch wie lautlich wäre wohl gegen eine solche Rechtschreibreform nichts einzuwenden. Allerdings liegt einem chinesischsprachigen Leser offenbar eine ,Vorliebe‘ tatsächlich schwer im Magen. Immerhin scheint auch gemäß dem ,Mund“ 口 (kǒu) in 嗜 (shi) für ,Geschmack finden an' eine ,Sucht‘ ( $p \grave{\imath}$ shi 癖嗜) gefühlsmäßig sehr wohl von den Essgewohnheiten auszugehen. 
Eine ,Fehlschreibung' der geschilderten Art - also etwa 僻 statt 癖 - wäre als eine Fehlschreibung im Bild zu werten. Mitunter verleitet ein Bildbewusstsein - genauer ein auf die Zeichengestalt bezogenes Sprachgefühl - zu einer sachlich fragwürdigen Schreibung. Die Wahl des Zeichens fußt im geschichtlichen Umgang mit ausdruckhaften Gestalten. Aufgrund dieses anderen Prinzips besteht ein signifikanter Unterschied zu einem von phonologisch-etymologischer Rationalität bestimmten ,orthographischen' Missgriff. Die angeführten Beispiele machen klar, wie die Schreibgestalt das Vorstellen des Lesenden an der verlautbarten Sprachebene vorbei mit Macht in die eine oder andere Richtung lenkt. Über die Wortbedeutung und das Phonem hinaus wird die jeweilige Verwendung in der Schrift bestimmt vom Gesichtspunkt der Zeichengestalt mit ihren eigenen Assoziationsfeldern. Anders herum wirkt aber auch der so oder anders konventionalisierte Zeichengebrauch zurück auf die Entwicklung der jeweiligen Konnotationen im Sprachgebrauch. Die gewohnheitsmäßige Anschauung unterschiedener Zeichenwörter, das heißt die Anschauung der unterschiedlichen Ausdrucksgestalten sprachlichen Sinnes in unterschiedlichen Zusammenhängen, färbt ihrerseits auf die Hermeneutik der betreffenden Sprachausdrücke ab. In diesem - von der Linguistik nicht hinreichend beachteten - Phänomen tritt die Kluft zwischen einem phonetisch erfassten Lexem und einem Zeichenwort als eine Grundtatsache der chinesischen Sprachpragmatik offen zutage.

Aufschlussreich sind des weiteren gebräuchliche Schreibvarianten für dasselbe Zeichenwort - ,abweichend' oder ,anders verkörperte Zeichenwörter' (yì tǐ zi 異體字, bié tǐ zi 別體字). So macht es anschauungsmäßig einen kleinen, aber feinen Unterschied, ob das Wort ,drinnen/Inneres ${ }^{6}$ als 裡 ( $l i$ ) oder als 裏 ( $l i$ ) geschrieben wird. Das ,Innenfutter' hängt ja nicht außen am,Gewand'衣 $(y \vec{\imath})$ - so wie 里 rechts neben dem Radikal 衣 beziehungsweise 衣 steht. Das Futter wird vielmehr handgreiflich umschlossen vom Gewand, sachlich gesehen befindet es sich an der ,Innenseite 裏 (li) desselben. Diesem Sachverhalt wird diejenige Schreibung bildhaft gerecht, die das ,Gewand'衣 vertikal in zwei Hälften zerteilt, in deren Mitte der lautanzeigende Bestandteil 里 Platz findet. So entsteht als Sinnbild das Zeichenwort 裏. Über treffend und weniger treffend entscheidet hier abermals die Gestaltanschauung. Diese richtet sich indes nicht auf ,Piktographisches', denn sie setzt Sprachkenntnis voraus - sowohl eine Kenntnis der Phonetik wie die derjenigen Grundregeln, die den Aufbau eines Schriftzeichens bestimmen.

Ähnlich verhält es sich mit einfacher oder komplexer ausfallenden stilistischen Schreibvarianten der Pinselschrift. Je nach Gestaltbildung 
machen diese Schreibformen mehr oder weniger deutlich sichtbar, was das betreffende Zeichenwort besagt. In dem Regelzeichen für ,schreiben' 書 $(s h \bar{u})$ ist über einem mit Inhalt gefüllten Mund, der etwas ,ausspricht ${ }^{\star}$ 曰 $(y \bar{u} e)$, deutlich das Schreibwerkzeug, der, Pinsel ${ }^{`}$ 聿 (yù), zu erkennen. In der verkürzten Variante 书, die seit zweitausend Jahren in der Schnell- oder ,Grasschrift‘ (căo sh $\bar{u}$ 草書) zum Einsatz kommt, fällt die Gestalt sichtlich abstrakter aus. Der,Wagen' 車 $(c h \vec{e})$ mit zwei klar erkennbaren Achsen vor und hinter der Ladefläche gemäß der bis heute gebräuchlichen ,Regelschrift' (kăi sh $\bar{u}$ 楷書) ist in seiner Struktur anhand der Grasschriftform 车 nur mehr zu erraten - wobei allerdings in der modernen Druckform zusätzlich Gestaltmomente des Pinselduktus unterdrückt werden.

Jeder Eingriff in die Zeichengestalt schließlich reicht tief in die Semantik hinein. Durch systematische Andersschreibung wird die Wahrnehmung infolge einer irreführenden Gestaltbildung mitunter gewaltsam in eine unangebrachte Assoziationsbahn gezwungen. Zahlreiche der heute auf dem chinesischen Festland vorgeschriebenen Vereinfachungen und Ersetzungen überlieferter Regelzeichen führen dies vor Augen. Entgegen der leitenden These von der konventionellen Bindung der chinesischen Schrift an Phoneme oder Wörter der Rede tritt heute die Krux einer gestalthaft Sinn entfaltenden Schrift drastisch zutage: Wo immer an die Gestalt eines Zeichenwortes Hand angelegt wird, hat dies unweigerlich Einfluß auf dessen Bedeutung. Aus manch überliefertem Regelzeichen ist nach der ideologisch gesteuerten Schriftreform ein anderes Wort geworden - bis hin zu echten ,Missbildungen‘.

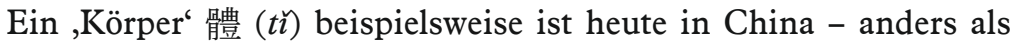
in Taiwan und Hong Kong - nicht nur seines Lautbestandteiles 豊 (lǐ), sondern auch seiner ,Knochen“ 骨 ( $g u ̛$ ) verlustig gegangen. Immerhin war hier der Bildertausch vielleicht so schlecht nicht: Ein ,Körper' 体 findet sich jetzt - wie in Japan - zusammengesetzt aus einem, Menschen'人 beziehungsweise $\uparrow$ (rén) und einem ,Stamm`本 (bèn). Der ,Körper' ist zum ,Stamm des Menschen' 体 mutiert - er mag organisch wachsen, besitzt dafür jedoch weniger inneren Halt als jener ältere ,Körper ${ }^{`} h^{6}$ 豊, der noch Knochen 骨 barg. ,Wolken'云 (yún, regulär 雲) haben verblüffenderweise den ,Regen'雨 ( $y \breve{u})$ eingebüßt und verdanken ihr Aussehen nun dem gleichlautenden Allerweltswort für, wörtlich'云 (yún). Wo in den überkommenen Gestalten des Zeichenwortes,Streit‘ 鬥 oder 鬪 (dòu) noch recht bildhaft zwei Kampfhähne aufeinander losgehen, wo in der alternativen Schreibung 鬫 sogar noch die,Streitaxt ${ }^{\star}$ 斤 (jinn) augenfällig ist, unterschlägt die Schriftreform all diese Assoziationen mit der Lehnschreibung 斗 (lies dòu). Nunmehr gleicht der ,Streit‘ aufs Ei einer 
simplen,Schöpfkelle‘ 斗 (dǒu). Die,Nudeln`麵 (miàn) folgten bisher aus guten Gründen dem Weizenradikal 麥 (mai) und waren damit auf jedem Ladenschild sogleich als Esswaren kenntlich. Heute dagegen grinst einen in der Volksrepublik China von Aushängen und Speisetafeln ein ,Gesicht' 面 (miàn) an. Dieses moderne Lehnzeichen ist in einer derartigen Verwendung nicht nur bedeutungsmäßig völlig irreführend; bereits beim Anschauen bedarf es einer gewissen Anstrengung, nicht an das Gesicht eines Menschen, vielmehr an eine Nudelsuppe zu denken.

Besonders das letzte Beispiel zeigt, welch groteske Verirrungen die von der europäischen Linguistik übernommene Doktrin vom Vorrang des Lautwortes gegenüber dem Zeichenwort in der chinesischen Schriftkultur anrichtet. Dort nämlich liest das Gestaltempfinden immer mit. Auch mögen die vereinfachten Schreibweisen leichter zu erlernen sein, doch stört es nachweislich den Lesevollzug, wenn etwa ein Lehnzeichen eine andere Aussprache suggeriert, oder wenn wichtige Gestaltmerkmale ausfallen und so die Sinnprägnanz in der Anschauung verringert wird. Ohne solche Gestaltmomente verkümmert ein Schriftzeichen zur suggestionsarmen Krücke. Aus einem physiognomischen Ausdruck voller Ausstrahlung wird ein konventioneller Platzhalter, der seine Bedeutsamkeit allein aus dem phonetischen Bestand und dem Kontext schöpfen muss. Andererseits schaffen es die ,neuen' Zeichen im Rahmen des jahrtausendealten Schriftsystems nicht, die Strahlkraft der Zeichengestalt restlos abzuschütteln. Nach wie vor bedeutet es einen Unterschied, ob wir nur hören, dass wir statt der ,Nudeln' ein ,Gesicht‘ verspeisen, oder ob wird dies schwarz auf weiß auf der Speisekarte lesen.

Die angeführten Belege gestatten es, ein erstes Resümee zu ziehen. Wer Chinesisches liest, schaut gewiss kein unmittelbar Sinn stiftendes Bild an. Allerdings entziffert er ebensowenig ein Codezeichen im Hinblick auf einen Sprachlaut und einen abstrakten Sinngehalt. Die allgemeine Betonung einer konventionellen Bildung der chinesischen Schriftzeichen übersieht, dass deren Gebrauchsgewohnheiten weder semantisch noch phonologisch hinreichend zu erklären sind. Vielmehr folgen sie bestimmten Sehgewohnheiten, mithin jenen bildmäßigen Assoziationen, die von der Zeichengestalt in der Vorstellung freigesetzt werden. Der Lesende begegnet in dieser Schrift physiognomisch ausdruckhaften Sinnbildern, die er bereits „in einer Art Mimetismus“ (une sorte de mimétisme) ${ }^{16}$ für sich übernommen hat, noch ehe ein hermeneutisches Verstehen im intellektuellen Sinne

16 Merleau-Ponty: Phénoménologie de la perception (wie Anm. 3), S. 378. 
einsetzt. Dass die leibliche Anrührung durch das Zeichenwort so selbstverständlich abläuft, dass sie im flüssigen Lesevorgang in aller Regel unbemerkt bleibt, daß auch nicht sämtliche Schriftzeichen die beschriebene Ausdruckskraft in gleichem Maße verkörpern, all dies soll nicht geleugnet werden. Es bedeutet jedoch keinen prinzipiellen Einwand gegen die hier verfolgte These: Im Aufbau der Schriftzeichen sind Gestaltmomente systematisch für das Sprachverstehen von Belang.

In Anbetracht der unmittelbaren Eröffnung von Vorstellungsfeldern durch den gezielten Einsatz der Denominativa in Literatur und Dichtung leuchtet schließlich ein, dass die gestaltassoziative Erschließung des Schriftsinnes sogar im philosophischen Denken unter Umständen eine nicht unerhebliche Rolle spielen kann. Gerade wo die schwerwiegende Homophonie des chinesischen Wortschatzes das knapp gesprochene Wort in nahezu unverständlicher Vieldeutigkeit verschwimmen lässt, haben Dichter und Denker seit jeher zum Mittel einer absichtsvollen Wahl der Radikale gegriffen. Dies geschah und geschieht nicht bloß, um den Sinn angemessen zu differenzieren und zu präzisieren; oft soll nachgerade ,auf einen Blick` eine größtmögliche Sinnfülle vermittelt werden.

\section{DENKEN ZWISCHEN WORT UND GESTALT}

Was zum einzelnen Schriftzeichen gesagt worden ist, lässt sich auf die Ebene ganzer Ausdrücke und Bedeutungszusammenhänge übertragen. Auch hier arbeitet im Innern der chinesischen Schrift dieselbe gestalthafte Assoziationswirkung und prägt nachhaltig das hermeneutische Erschließen eines Textes - bis in die philosophischen Grundentscheidungen hinein. Der Zusammenhang zwischen paıvó $\mu \varepsilon v o v$, apparitio und ,Erscheinung' setzt ein begriffsgeschichtliches Spezialwissen voraus. Wo in der Reihe ,Erscheinung', ,Schein', Scheinen' begriffliche und wertmäßige Differenzierungen mitschwingen, leuchten diese geschichtlichen Scheidungen vom Schriftbild allein her nicht ein. Vermittels wirksamer Gestaltmomente leuchten im Chinesischen hingegen manche Bedeutungen schon in der Anschauung unmittelbar ein. Jedes Schriftzeichen eröffnet ein Geflecht von Assoziationen, die der allgemeinen sprachlichen Semantik entspringen, ihren vollzughaften Ort indes auf der Ebene unmittelbarer Anschauung haben.

Während das lateinische Wort substantia allenfalls dem Etymologen Aufschluß gibt über das ursprünglich Gemeinte, eröffnet die chinesische Übersetzung shit $t$ 實體 schon im Leseakt bestimmte Sinnhorizonte. Noch 
vor philosophiegeschichtlichen Festlegungen bringt im Schriftbild der Substanzbegriff einen ,voll' ( $s h i$ 實) erfüllten ,Körper' ( $t \imath$ 體) mit seinem ,Knochengerüst' ( $g \check{u}$ 骨) zum Austrag. Wo in ,humanitas' der ,homo', in ,Menschlichkeit' der ,Mensch' nachhallt, da erfasst der Chinesisch Lesende diese Bedeutung mit den Augen: Rén 仁 ist etwas, was sich zwischen zwei 二 (èr),Menschen'人 (rén) beziehungsweise $\uparrow$ abspielt. Diese gestalthafte Bedeutungsassoziation mag philosophisch sinnvoll sein oder nicht; allein, sie lässt sich bei der Lektüre gar nicht neutralisieren. Ein chinesischer ,Leibkörper' oder ,Leib', shēntǐ 身體, verkörpert im Zeichenwort 身 (shēn) die leibliche Fülle des ,Rumpfes', in 體 ( $t \grave{\imath}$ ) den ,gegliederten Zusammenhalt der Knochen'. Mit einem physischen oder unbelebten ,Körper' (wù̀tǐ 物體) teilt dieser Leibbegriff den, gegliederten Zusammenhalt' ( $t \imath$ 體); doch einen ,Leib‘ hat eben nur, wer ,Person` und ,Selbst' - ebenfalls 身 (shēn) - sein kann, im Unterschied zum bloßen ,Körper'物體 von,Vieh und Dingen' (wù 物). ,Verstehen' tritt sinnfällig als ,leibhaftig übereinkommen' (tǐhuì 體會) oder ,leibhaftig innesein' (tìzwù 體悟) auf, zuverlässiges Wissen als , leibhaftig erkennen' (tìrèn 體認) und ,leibhaftig wissen' ( $t \grave{\imath z h} \bar{\imath}$ 體知). Gerade eine Leibphänomenologie kann also in dieser Schrift ihre Gesichtspunkte und Entdeckungen unmittelbar in den geschriebenen Ausdruck heben.

Zwischen dem Schriftbild, Wahrheit' und dem Schriftbild ,Rationalität ${ }^{`}$ drängt sich in der Anschauung keinerlei Verbindung auf. Hingegen ist im Chinesischen das Band zwischen den modernen Termini für, Wahrheit' (zhênlı̌ 真理) und ,Vernunft' (lǐxing 理性) nicht zu übersehen: Als die ,echten Bahnen der Wirklichkeit' (zhên lǐ 真理) bahnt die Wahrheit der, auf die Bahnen der Wirklichkeit angelegten Natur' (lí xing 理性), also der Vernunft, den Weg. ,Bahnen der Wirklichkeit' ( $l$ ľ 理) bestimmen das Weltgeschehen wie das Denken. Darüber hinaus holt dieses 理 Facetten älteren Denkens in die moderne philosophische Sprache herein. Im Horizont von, Wahrheit' und ,Vernunft' tauchen anschaulich weitere Ausdrücke auf, etwa dàolı̌ 道理 für ,Sinn', tiānlı̌ 天理 für ,Naturgegebenheit', lúnlı̌ 倫理 für, Sittlichkeit'. Der Anrührung durch die mit Geistesgeschichte auratisch angereicherte Zeichengestalt des 理 wird der Lesende sich kaum entziehen können; so wird schon auf der sinnlichen Anschauungsebene die Geistesgeschichte erneut denkerisch wirksam. Der prägnante Gestalthorizont des Zeichenwortes leitet nicht nur die Suche nach sachlichen Zusammenhängen an, er kann als anschauungsmäßige Affektion von der philosophischen Denkebene unmittelbar bis ins Leibsein ausstrahlen. In dieser Hinsicht bietet zuletzt der Blick auf den Schreibakt entscheidende Aufschlüsse. 


\section{SCHRIFT UND GESTISCHER AUSDRUCK}

Von Friedrich Nietzsche stammt die Bemerkung, man lese mit den Muskeln. ${ }^{17}$ Genau dies, ein leibliches Lesen und Denken, scheint in der Tat die chinesische Schrift zu fordern und zu fördern. Appellativ stößt sie im Lesenden leibliche Modulationen von existenzieller Tiefe an. Nicht nur in einzelnen Gestaltkomponenten eignet dem Zeichenwort eine assoziative Strahlkraft; auch als Gestaltganzheit ist es auf komplexe Weise durchdrungen von einer affektiven Wirkmacht. Durch eine rund zwei Jahrtausende währende Kulturentwicklung hindurch hat sich das Heer der Schriftzeichen kontinuierlich die menschliche Schreibarbeit einverleibt. Aus dem Schreibakt hat die Zeichengestalt leibliche Bewegungsqualitäten in ihre Tiefenstruktur aufgenommen, weshalb sie nur aus den zugehörigen Aktvollzügen heraus durchsichtig gemacht werden kann.

Obgleich gedruckte Texte in China schon seit dem siebten Jahrhundert Verbreitung fanden, hat das Schreiben mit Pinsel und Tusche den Umgang mit den Schriftzeichen entscheidend geprägt. Diese Schrift wird nach wie vor weniger als starres Abbild des gesprochenen Wortes denn viel eher als lebendige Gebärde wahrgenommen. In Aufbau und Ausführung der Schriftzeichen sieht der Eingeweihte die Spur einer menschlichen Ausdrucksgeste; bis in winzigste Nuancen hinein findet die Schreibberwegung darin ihren anschaulichen Niederschlag. Eine von leiblichen Bewegungsimpulsen und -empfindungen getragene Abfolge der Pinselstriche (b̌̌ shùn 筆順) bestimmt nach wie vor die Grundordnung jedes einzelnen Zeichens wie die eines Schriftzuges. Das Pinselschreiben ist bis in die Drucktypen der Gegenwart hinein ein integraler Bestandteil des Schriftsystems. Aus diesem Grund bedeutet die Umstellung der Schreib- und Druckrichtung in die Horizontale von links nach rechts - statt vertikal von rechts oben nach links unten eine politische Maßnahme buchstäblich, gegen den Strich' der Schrift. Ebenso verfehlt ist es, in der Frage nach der Gestaltwahrnehmung beim Lesen die Pinselschrift als ein überholtes Kommunikationsmedium oder als handschriftliches Beiwerk abzutun. Vor allem die Pinselschrift bietet

17 Vgl. Friedrich Nietzsche: Kritische Studienausgabe. Hrsg. von Giorgi Colli und Mazino Montinari. 15 Bde., 2., durchges. Auflage, München: DTV 1988, Bd. XIII: Nachgelassene Fragmente 1887-1889 (Nachlass Frühjahr 1888) 14 [119], S. 296. 
wertvolle Einblicke zum Zusammenhang zwischen Schriftgestalt und leiblich involviertem Denken.

Statt von einer fertigen ,Form' des Schriftzeichens zu sprechen, ist dessen Aussehen vielmehr grundsätzlich als eine ,Bewegungs- und Zeitgestalt‘ (shi 勢) aufzufassen. Das Zeichen ist weniger das Ergebnis einer Niederschrift, sondern eine materialisierte Gestaltwerdung in der Zeit. Unweigerlich tritt stets ein bestimmter Schreibduktus in die Erscheinung. Jedes einzelne Schriftzeichen verkörpert in ausdruckhafter Weise eine Bewegungsgebärde - die ,Bewegungs- und Zeitgestalt der Pinselführung (bi shi 筆勢). Diese fügt dem Schriftzug nicht lediglich eine individuelle Note hinzu, sie ist systematisch in die Verfassung der Schriftzeichen selbst integriert. Da die Erfordernisse des Pinselschreibens in die Struktur der Schrift Eingang gefunden haben, besteht ein wesensmäßiger Unterschied zwischen der über rund zwei Jahrtausende hinweg ausgeprägten, bis heute unverändert den Gipfel kultureller Bildung markierenden chinesischen ,Schreibkunst' $\left(s h \bar{u}\right.$ fa 書法) ${ }^{18}$ und dem, was anderswo als ,Kalligraphie gepflegt wird. Bis in die Schreibtypen des Computerzeitalters hinein entspringt die chinesische Schrift in ihrem Kern dem verfeinerten $\mathrm{Zu}-$ sammenspiel von Handbewegung, Pinsel, Tusche und Schreibunterlage. Das Schreiben mit dem Pinsel dient der Mitteilung, doch im Gebrauch setzt es stets einen aisthetischen Überschuss frei, der sich gleichermaßen auf das Schriftbild und die Person des Schreibenden bezieht. Im Einsatz der Schrift kann daher aus dem gestalthaften Wesen der Schrift selbst eine ästhetisch bedeutsame, Schreib-Kunst' erwachsen; diese wiederum kann im Rahmen eines lebenslangen Bildungsweges der Person sogar zur ,Lebensübung' werden. Oftmals wurde und wird die Schreibkunst in diesem Sinne praktiziert als ethische Einübung des einzelnen in ein Weltverhältnis und eine geschichtliche Gemeinschaft. ${ }^{19}$

Im wahrnehmungsmäßigen Rückbezug des Geschriebenen auf den Akt des Schreibens vollzieht sich noch etwas ganz Wesentliches. Oftmals sagt jemand etwas, vermag es aber im Moment nicht niederzuschreiben; er verfügt über das Wort, aber nicht über dessen Zeichengestalt. Um sich zu erinnern, muss er in seiner Vorstellung eine physiognomische

18 Siehe die Gesamtdarstellung von Jean François Billeter: L'art chinois de l'écriture. Essai sur la calligraphie. Genf und Mailand 1989/2001.

19 Siehe Mathias Obert: Leibliche Mimesis und Selbstsorge in den chinesischen Künsten des Pinsels. In: Marcus Schmücker und Fabian Heubel (Hrsg.): Dimensionen der Selbstkultivierung. Beiträge des Forums für Asiatische Philosophie. Freiburg i. Br. und München 2013, S. 399-430. 
Assoziationskette durchlaufen, das heißt er muss - sei es unter Berücksichtigung derselben Aussprache, sei es aufgrund ihm gegenwärtiger Gestaltmerkmale - eine Abwandlung möglicher Zeichengestalten vornehmen. Nicht selten wird ihm dabei die Erinnerung wiederkehren, sobald er nochmals zum Schreiben ansetzt. Wonach er sucht, ist weniger eine Figur, ist viel eher eine Schreibweise, mithin der Akt einer Figuration. Er muss sich leibhaftig in eine leibliche Schreibgeste hineinversetzen, um das Vorstellungsbild von dem Zeichen erneut in sich zu erzeugen. Bisweilen ist dieses auch im Aufbau so komplex, dass er es nicht vollständig in simultaner Vorstellung als Figur vergegenwärtigen kann. Doch es genügt, wenn er in der Phantasie die Schreibbewegung mit den tauglichen Impulsen und Motivationsketten anstößt, um das Zeichen ohne Zögern auszuschreiben. Indem er bis in seine leibliche Haltung und seine leiblichen Bewegungsabläufe hinein eine Mimesis der entsprechenden ,Bewegungs- und Zeitgestalt' (sh $i$ 勢) vollführt, stellt sich das gesuchte Zeichen wieder ein. Diese alltägliche Beobachtung liefert im übrigen eine erstaunlich stimmige Bestätigung jener Analysen zur Verwurzelung des Denkens und Sprechens in leiblichen Situationen und Bewegungsvollzügen, die Merleau-Ponty anhand von Aphasien und anderen krankhaften Fehlfunktionen vorführt. ${ }^{20}$

Traditionell charakterisieren nicht von ungefähr anthropomorphe Resonanzen wie ,Knochenbau' (gŭ 骨) und ,Fleisch' (ròu 肉) die Gesamtqualität eines Schriftzuges, denn über eine Jahrtausende hindurch fortgesetzte Schreibübung sind Dimensionen des menschlichen Leibseins zu einem integralen Bestandteil des Schriftsystems geworden. Ästhetisch geben leibliche oder leiblich empfundene Momente wie Anstrengung und Kraft, Berührung und Widerständigkeit, Material- und Formempfinden, Rhythmus, Tempo, Dicke der Linie, satter Farbton und trockener Auftrag den Ausschlag. ${ }^{21}$ Weit über eine bloße Kenntnis oder Fertigkeit hinaus unterhält infolgedessen der Chinesisch Lesende, der zugleich Schreibender sein muss, eine tief in sein leibliches Selbst hineinreichende Beziehung zu den Schriftzeichen. Sein Gebrauch der Schrift ist vorrangig eine Begegnung mit der leiblichen Strahlkraft ihrer Gestalten. Vermittels einer oft lebenslangen leiblichen Arbeit an sich selbst hat er das Schreiben

$20 \mathrm{Vgl}$. Merleau-Ponty: Phénoménologie de la perception (wie Anm.3), S. 222-230 und 272-274.

21 Siehe Mathias Obert: Tanzende Schrift. Beobachtungen zur chinesischen Schreibkunst. In: Antonio Loprieno, Carsten Knigge Salis und Birgit Mersmann (Hrsg.): Bild, Macht, Schrift. Schriftkulturen in bildkritischer Perspektive. Göttingen 2011, S. 215-239. 
mit dem ebenso sensiblen wie schwer zu meisternden Schreibmaterial habitualisiert. Dabei hat er die Schreibbewegungen in seine leiblichen Bewegungsmuster eingeschrieben; zugleich hat er in der mimetischen Auseinandersetzung mit in den Schriftvorlagen geschichtlich verkörperten sozio-kulturellen Mustern einen leiblichen Bezug zur Welt aufgebaut, welcher in alle Regionen seines Verhaltens hinein ausstrahlt. Der Schreibende vollzieht buchstäblich eine ,Einleibung' der Schrift, auf diesem Wege jedoch zugleich die eigene ,Einschreibung' in eine mit anderen geteilte Kulturwelt. Aus den Ausdruckswerten der Schrift treten dem Lesenden diese leibliche Anstrengung und diese Leibzeit in einer geschichtlichen Gemeinschaft entgegen. Die Gesamtheit der beim Schreiben habitualisierten Bewegungsmomente und Empfindungswerte wird bei der Lektüre in leiblicher Mimesis nachvollzogen. Wo die Gestalt der Zeichenwörter, mithin das Grundelement von Sprache und Denken, in der beschriebenen Weise über das Schreiben mit dem Pinsel mit einer unerhörten Fülle an leiblicher Wirkmacht angereichert ist, wird diese in der lesenden Wahrnehmung mit dem sanften Zwang eines Habitus erneut in leibliche Impulse umgesetzt.

Traditionell wird angesichts des Ausgeführten der regelhafte Aufbau

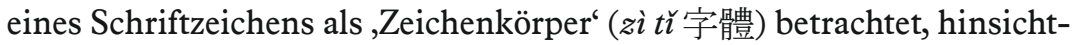
lich des physiognomischen Ausdrucks einer Schreibart spricht man vom ,Schreibkörper' ( $s h \bar{u} t \grave{t}$ 書體), hinsichtlich einer literarischen Stilgattung vom ,Körper des Geschriebenen' (wén tǐ 文體). Da drängt sich die These von einer der Zeichengestalt immanenten strukturellen Leiblichkeit nachgerade auf. Diese wiederum führt dazu, dass leibliche Zuständlichkeiten und Affektionen das semiotisch-semantische Erschließen eines Textes jederzeit begleiten, sofern sie ihm nicht sogar vorhergehen. Zusammenfassend läßt sich daher feststellen: Einerseits bietet die chinesische Schrift dem Lesenden gefühlsgeladene Anschauungsinhalte dar, die eine bildhafte Vorstellungstätigkeit anstoßen und die, je nach Kontext und Stilebene, in mehr oder weniger appellativer Weise das Denken leiten. Der Lesende kann sich einer anschauungsmäßigen Anrührung durch die Gestaltintensität der Zeichen nicht in gleicher Weise entziehen wie im Falle einer Lautschrift. Andererseits setzen die Schriftzeichen beim Lesen analog zum Schreibakt leibliche Empfindungen und Bewegungsimpulse frei. Zumal angesichts der lebendigen Physiognomie mit dem Pinsel geschriebener Zeichen wird die Lektüre begleitet von einem leiblich mitschwingenden Ausdrucksempfinden. Das Zeichenlesen lässt sich also keinesfalls auf eine passive Aufnahme von Sprachsinn durch den Verstand reduzieren. Die Sinnstiftung beim Lesen vollzieht sich vielmehr in Form eines Antwortens auf eine gestalthaft-leibliche Gesamtaffektion. 


\section{FÜR EIN GESTALTHAFT LEIBLICHES DENKEN}

Befördert ein chinesisch geschriebener Text, befördert die mit gestalthaft wirksamen Schriftzeichen symbiotisch verbundene chinesische Sprachlichkeit eine Art leibliches Denken? Ein solches Denken ,durch die Leiblichkeit hindurch' wäre allerdings aufgrund seiner engen, ja sein Wesen berührenden Verflochtenheit mit einer gestalthaften Schrift, wohl zu unterscheiden von der jüngeren philosophischen Thematisierung des Leibes in Europa wie ebenso von ,somaästhetisch' gesteigerter Selbstwahrnehmung. ${ }^{22}$ Auf der Grundlage der chinesischen Schrift hat sich die Leiblichkeit nämlich je schon im Denken eingenistet, das Denken wird da durch die Brille der Schriftzeichen prinzipiell dazu angehalten, die Welt und sich selbst immer in leiblichen Bewegungsgestalten zu erfahren und zu denken. Dass dies durch die Geschichte hindurch zumeist insgeheim geschah und in aller Regel nicht eigens thematisiert wurde, tut den hier gezogenen Schlußfolgerungen keinen Abbruch. Bis in die jüngste Zeit hinein fehlte ja dem chinesischsprachigen Denken schlicht die Alternativerfahrung von einer gewissermaßen leibentleerten, abstrakten Lautschrift, so dass die hier vorgetragenen kritischen Überlegungen vermutlich schon allein deshalb bisher so kaum angestellt werden konnten. Zum anderen muss betont werden, dass mit diesem Beitrag das chinesischsprachige Denken beileibe nicht auf Leiblichkeit als Generalthema fixiert werden soll; aufgewiesen werden sollen lediglich Anlagen und Möglichkeiten; ob sie genutzt werden oder brachliegen bleiben, entscheidet jeder Denkweg selbst.

Aus den oben erörterten Gesichtspunkten leitet sich in Zuspitzung

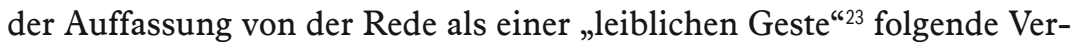
mutung ab: Sofern ein chinesischsprachiges Philosophieren in Geschichte und Gegenwart unweigerlich auf der Schriftlichkeit und einer damit verbundenen Anschauung konkreter Gestalten beruht - und dies in wesentlich höherem Maße als die europäische Philosophie -, insofern dringen über das sprachliche Ausdrucksverhalten allenthalben leiblich fundierte Motivationen und Zustände unmittelbar in den Bezirk des Denkens ein.

22 Vgl. Richard Shusterman: Thinking through the Body. Essays in Somaesthetics, Cambridge 2012.

23 Merleau-Ponty: Phénoménologie de la perception (wie Anm. 3), S. 208, 211, 214, 218-219. 
Chinesischsprachiges Denken unterhält infolge der Eigentümlichkeit seiner Schrift - allen Abnutzungs- und Gewöhnungserscheinungen zum Trotz - eine weitreichende Verbindung mit der leiblichen Dimension des Menschseins. Indem dieses Denken sich vermittels anschaulich reicher Audrucksgestalten ausbildet, muss es sich nicht nur leibhaftig einlassen auf das Gedachte; es wird immer wieder von seinen sprachlichen Denkräumen, welche die Schrift als einen unverzichtbaren Bestandteil implizieren, dazu verführt oder sogar gezwungen, mit dem Leib und durch den Leib hindurch, im Rückgang auf die leiblich-existentielle Bewegung des Lebens zu denken.

Über die verschiedenen Ebenen der Gestaltassoziation - von Gegenständen über Vorstellungsbilder bis hin zu gestischen Empfindungswerten und Bewegungserfahrungen - zeichnet die chinesische Sprachlichkeit bis in die Texthermeneutik hinein eine leibliche Tiefe aus. Schon auf der Ebene der Anschauung gestaltet sich die Textlektüre als ein mit leiblichen Wertigkeiten aufgeladenes, als ein leiblich angerührtes und leibhaftig antwortendes Verstehen von sprachlichem Sinn. Wo die konkrete Gestaltfülle in der Anschauung bildhafte Denkfelder eröffnet, wo überdies die gestalthafte Intensität der Pinselschrift im leibmimetischen Nachvollzug unmittelbar einwirkt ins leibliche Selbst des Denkenden, da wird sein Denken sich der lebendigen Bindung an sein Leibsein schwerlich entziehen können. Wie der heideggersche Riss zieht sich eine Art ,leibliche Falte' durch die gesamte sprachliche Denkarbeit hindurch und gebiert philosophische Einsicht.

An dieser Stelle ist endlich eine kritische Frage angebracht: Bedeutet die leibliche Gestaltfülle chinesischer Sprachlichkeit nicht eher eine Gefährdung oder ein Hemmnis für das philosophische Denken? Birgt diese eigentümliche Gestalthaftigkeit tatsächlich Anregungen für ein echtes Philosophieren, oder lenkt sie nicht vielmehr vom begrifflich scharfen Denken ab? Selbstverständlich lassen sich solche Zweifel nicht einfach ausräumen. Immerhin kann jetzt aber eine strukturelle Disposition chinesischsprachigen Denkens Aufmerksamkeit finden, die fruchtbare Bezüge zu namhaften Positionen der europäischen Gegenwartsphilosophie aufweist. Der alte Zweifel an der Tauglichkeit der chinesischen Sprache und Schrift für die Philosophie lässt sich so in einen philosophischen Antrieb umwandeln. Das zeitgenössische Denken in chinesischer Sprache ist tief in seiner eigenen Sprachlichkeit und unerhört reichen literarischen Überlieferung beheimatet; zugleich aber ist es bereits zu einem Moment innerhalb der Gegenwartsphilosophie geworden. Aus diesem Grund sollte der neue denkerische Ort, den chinesischsprachiges 
Denken heute eröffnet, als ein wichtiges Potential wahrgenommen und genutzt werden. ${ }^{24}$

Zur reizvollen Auseinandersetzung mit chinesischsprachigem Denken in der Gegenwart trägt die Herausforderung durch eine nicht-europäische Sprachlichkeit und Schriftlichkeit erheblich bei. Ein im ,Umweg über China' transkulturell sensibilisiertes Nachdenken wird nicht nur einen gewichtigen Beitrag zur Revision eurozentrischer Axiome der Linguistik und der Sprachphilosophie leisten. Die Gestaltdichte der chinesischen Schrift lädt zu einer Vertiefung gestaltpsychologischer und symboltheoretischer Ansätze oder zur weiteren Ausarbeitung leibphänomenologischer Beschreibungen ein. Von hier aus lässt sich Hans Blumenbergs Ansetzen bei der Lesbarkeit der Welt fruchtbar vertiefen, erschließt doch ein in chinesischer Schrift verfasster philosophischer Text bereits auf der Anschauungsebene welthafte Vorstellungs- und Erlebnishorizonte. Bereits vor jeder sprachlichen Verlautbarung findet da in den kulturellen Räumen der Schriftlichkeit eine leiblich erfahrene Durchdringung von Welt, Mensch und Sinn statt, die für europäisches Denken nicht leicht nachzuvollziehen, geschweige denn pragmatisch einzuholen scheint. Maurice Merleau-Ponty beschreibt und analysiert einen derartigen $\mathrm{Zu}-$ sammenhang zwischen Sprache und Welt sehr treffend unter dem Stichwort des „anthropologischen Raumes“ (espace anthropologique). Wenn ihm zufolge ein physiognomisches, das heißt ein auf Affektivität und Ausdruck gerichtetes mythisches Weltverständnis den „erwachsenen“, objektivierenden und zuletzt wissenschaftlichen Weltzugang fundiert, dann bedeutet dies, dass auch jede noch so abstrakte Bild- und Sprachgestalt letztlich je schon in leiblicher Bewegung, in einem leiblichen Verhalten zur Welt gründet. ${ }^{25}$ Sofern nun die chinesische Schrift - entgegen den Annahmen eines Leibniz - nicht etwa das Reich der Erkenntnis in ,Ideogrammen' schematisch abbildet, wenn sie viel eher eine physiognomisch wirkende Gestaltwelt zum Austrag bringt, dann muss doch bis in die lesende Anschauung dieser Schriftgestalt hinein das leibliche Verhalten

24 Siehe Mathias Obert: Begegnung mit der chinesischsprachigen Welt heute. Weder komparativ noch interkulturell: In: Zeitschrift für Kulturphilosophie 3.1 (2009), S. 313-321; Mathias Obert: Interkulturalität und philosophische Grundfragen? Polylog im chinesischsprachigen Denken der Gegenwart. In: F. G. Pranzl und A. Graneß (Hrsg.), Perspektiven interkulturellen Philosophierens. Beiträge zur Geschichte und Methodik von Polylogen: Für Franz Martin Wimmer. Wien, S. 341-355.

25 Merleau-Ponty: Phénoménologie de la perception (wie Anm. 3), S. 324-344. 
des Menschen zur Welt die zentrale Sinnachse liefern. Daraus ergeben sich wenigstens zwei philosophische Perspektiven.

Zum einen scheint chinesischsprachiges Denken strukturell eine gänzlich andere Stellung zu Grunddispositionen der abendländischen Metaphysik einzunehmen - oder zumindest einnehmen zu können. Einen Schlüssel dazu sehe ich in der leiblichen Dimension wie in einer Bildhaftigkeit von Schrift und Sprache, die gerade nicht mit einer Abbildtheorie und der klassisch platonistischen Zweiteilung des Wirklichen übereinfällt. An der systematischen Stelle, an welcher in Europa die moderne Krise und der Nihilismus zum Ausbruch gelangt sind, scheint im chinesischsprachigen Denken eine ungehemmte Weltzugewandtheit tief im soziokulturellen Element von Sprache und Schrift verwurzelt zu sein. Zumal die Schrift bewahrt dem Denken seinen Bezug zur Lebenswelt.

Sodann sehe ich in einer aisthetischen Dimension philosophischer Texte, die in ihrer Schriftlichkeit selbst gewissermaßen quer zum aristotelischen Schema verfasst sind, die wertvolle Möglichkeit zu einer Neubesinnung auf die pragmatische Dimension echten Denkens. Indem wir während des Lesens einen Text in seinen Gestaltwerten leibhaftig aufnehmen, erfahren wir seine Anrührung bis ins Innerste unserer existentiellen Bemühung um Sinn hinein; unser Verstehen und unser Denken kann sich von diesem Angerufensein her als ein lebendiges, im Sinne Heideggers als ein,wesentliches' Antworten ausbilden. Damit kann aber das hermeneutische Studium philosophischer Texte zu einer Denkübung werden, die bereits in der lesenden Anschauung einsetzt, um den Denkenden leiblich zu ergreifen und existentiell zu wandeln. Immer neu kann diese philosophisch-hermeneutische Übung über ihre Verwurzelung in einem leiblichen Denken zurücklaufen in die lebendig erfahrene Sinngestalt - zurücklaufen in die Gestalt von Geschriebenem und Gesprochenem als einen ,eingeleibten' Ausdruck von Welt. 



\section{WELT DER BUCHSTABEN}





\section{VERSUCH EINER PHÄNOMENOLOGIE DES BUCHSTABENS}

Das Konzept des Buchstabens ist in philosophischen Erörterungen weniger präsent als man zunächst meint. Wesentlich stärker hat sich das philosophische Denken am verwandten Begriff des Zeichens, der Schrift ${ }^{1}$ oder der Sprache abgearbeitet. Der Begriff des Zeichens (und dies gilt auch für Schrift und Sprache) beinhaltet bereits die Dimension der Bedeutung, er weist bereits auf die eine oder andere Art über sich hinaus. Das Zeichen ist ein Zeichen für etwas oder ein Anzeichen von etwas. Die Referentialität ist dabei nicht wegzudenken. Die Schrift stellt eine Fixierung eines Bedeutungszusammenhangs dar und bringt die Dimension der Wahrnehmbarkeit und Bildlichkeit hinein, welche über die reine Darstellung des Gesprochenen hinausweisen. Der Begriff der Sprache umfasst das gesamte Ausdrucksgeschehen, die universelle Bewegung und Generierung von Bedeutung.

In diesem umfassenden Geschehen bedeutsamen Ausdrucks scheint der Buchstabe auf einer niederen Ebene angesiedelt zu sein, weil er für sich genommen noch nicht über sich hinausweist, noch bedeutungslos bleibt. Buchstaben sind, um es mit einem Konzept Maurice MerleauPontys zu formulieren, das Fleisch der sprachlichen Bedeutung. ${ }^{2}$ Sie sind das Element, in dem die Worte entstehen. Daher können sie philosophisch nicht bedeutungslos sein. Ich möchte versuchen im ersten Teil dieses Beitrages dem Wesen des Buchstaben phänomenologisch nachzugehen. Dafür werde ich eine Analogie zwischen dem Buchstaben und

1 Dazu beispielsweise das Graduiertenkolleg von Sybille Krämer: Schriftbildlichkeit: Über Materialität, Wahrnehmbarkeit und Operativität von Notationen (DFG).

2 Vgl. Maurice Merleau-Ponty: Das Sichtbare und das Unsichtbare. München ${ }^{2} 1994$. 
Bildern (vor allem dem, was man in der phänomenologischen Bildtheorie die Bildmaterie nennt) herstellen. Im zweiten Teil werde ich dann der Spur des Buchstaben bei Merleau-Ponty folgen, um abschließend kurz auf Jacques Derridas Begriff der différance einzugehen.

\section{ANNÄHERUNG AN DAS KONZEPT DES BUCHSTABEN}

Der Buchstabe steht nicht am Anfang der Sprachentwicklung. Die Geste, der Laut, die Rede sind die Ursprungsphänomene der Sprache. Dennoch kann man auch den Buchstaben als ein Ursprungsphänomen betrachten. Denn er steht am Beginn der Objektivierung der ursprünglich an den subjektiven Vollzug des Sprechens gebundenen Sprache. Erst mit dem Aufkommen eines Notationssystems für sprachliche Laute bzw. Zeichen (dies gilt sowohl für Laut- als auch für Bildschrift) kann Sprache fixiert und damit ihrer Bindung an die Gegenwart des Sprechers enthoben werden. Sie erhält eine Dauer, die über die Zeitlichkeit des Sprechakts und in vielen Fällen über die des Sprechers selbst hinausreicht. Man könnte soweit gehen, zu sagen, dass die Sprache durch die Buchstaben verkörperlicht und verräumlicht wird. Die immateriellen, vergänglichen Lautgebilde erhalten so eine Gestalt. Über die Gestalt hinaus impliziert die Buchstabenschrift die prinzipielle Möglichkeit der Wiederholung einer jeden Bedeutung. Bedeutung durch Buchstaben fixiert erhält also nicht nur Dauer sondern wird zum Gegenstand immer möglicher Wiederholbarkeit. ${ }^{3}$ Damit wird die Bedeutung vom aktuellen Vollzug des Denkens und Sprechens abgelöst, sie wird gewissermaßen ursprungslos, denn das geschriebene Wort weist keine Spuren des Sprechers mehr auf (es sei denn, man betrachtet handschriftliche Notationen). Die Originalität verlagert sich vom Sprechakt zum Sprachstil. Nur stilistisch kann der Autor noch seine Signatur im Text hinterlassen, seine Anwesenheit wird für die Bedeutung bedeutungslos, wie auch seine Interpretation zu einer von vielen möglichen werden wird.

Die Wiederholbarkeit, und das möchte ich hier mit Derrida festhalten, ist jedoch keine Wiederholung von Identischem. Weil Verstehen von Sinn immer an einen größeren Zusammenhang gebunden ist, der durch das Fortschreiten der Zeit dynamisch, d. i. historisch veränderlich ist, wird auch die Bedeutung in ihrer Wiederholung beständig veränderlich

3 Vgl. Jacques Derrida: Differenz und Wiederholung. München ${ }^{3} 2007$. 
sein. Derrida kritisiert die Vorstellung der Präsenz eines Sinns, der durch die gesprochene Sprache direkter zugänglich wäre (Logozentrismus) als in der Vermittlung durch die Schrift. Jeder Sinn, jede Bedeutung ist immer schon sprachlich oder schriftlich vermittelt, es gibt sie nicht in Reinform.

Buchstaben sind Zeichen für Laute und Lautstrukturen. Damit ist doch bereits eine, wenn auch rudimentäre Verweisungsstruktur impliziert: Sie stehen für bestimmte Laute, diese sind wiederum die Elemente aus denen sich Sprache aufbaut. Im Falle der Lautschrift konstituieren die Lautzeichen die größeren Bedeutungseinheiten, die Worte. Interessant hierbei ist, dass die Buchstaben wie ein Material, wie z. B. die Farben eines Bildes funktionieren. Sie bilden die Struktur, die die Bedeutung trägt, ohne selbst thematisch zu sein. Der Normalfall der Lektüre ähnelt dem Normalfall einer Bildbetrachtung: Wenn ich einen Satz lese, bin ich intentional auf seinen Gehalt, auf seine Bedeutung gerichtet. Betrachte ich ein Bild, dann bin ich intentional zunächst auf den Gegenstand, das Bildsujet, um mit Husserl zu sprechen, ausgerichtet. ${ }^{4}$ Die Bildmaterie, der Bildträger, die konkreten Farben stehen in diesem Fall nicht im Fokus der Aufmerksamkeit. Sie werden gewissermaßen opak, denn durch sie hindurch ist der Betrachter auf den abgebildeten Gegenstand gerichtet. In einem idealen Bild wäre die Bildmaterie nicht nur opak, sondern vollständig transparent. Sie würde dem Blick kein Hindernis entgegenstellen auf dem Weg zum Gegenstand (man denke zum Beispiel an eine vollständige virtuelle Realität).$^{5}$

Anders als bei Bildern gibt es bei Buchstaben eine klare Grenze der Transparenz: Die fundamentale Unähnlichkeit zwischen den Lauten und ihren Zeichen lässt sich nicht auslöschen. Buchstaben werden als Erscheinung immer auf die notwendige Arbitrarität des sprachlichen

4 Vgl. dazu Edmund Husserl: Phantasie und Bildbewußtsein (1904/05). In: ders.: Phantasie und Bildbewußtsein. Hrsg. von Eduard Marbach. Hamburg 2006, S. 3-110; Lambert Wiesing: Phänomenologie des Bildes nach Edmund Husserl und Jean-Paul Sartre. In: ders.: Phänomene im Bild. München 2007, S. 43-6o.

5 Dann jedoch gäbe es laut Husserl auch kein Bildbewusstsein mehr. Das Bewusstsein davon, ein Bild zu sehen, hat gerade jenen Widerstreit zur Bedingung, der bei der Betrachtung entsteht: Nämlich jenem zwischen dem Bewusstsein vom Bild als materiellem Objekt und dem Bewusstsein vom Bildsujet als immateriellem Gegenstand, der im Bild zur Erscheinung kommt. 
Zeichens verweisen. Denn nicht nur haben Begriffe keine Ähnlichkeit mit dem, was sie bezeichnen, auch die Buchstaben haben keine Ähnlichkeit mit den Lauten, die sie repräsentieren. Obschon die prinzipielle Unähnlichkeit zwischen dem Laut und seinem Schriftzeichen ein Grund ist, warum Schriftzeichen nie ganz transparent sein können, findet sich jedoch auch eine Grenze hin zum Gegenteil, zur Opazität. Gerade weil das Schriftzeichen dem Laut so unähnlich ist, rückt es auch kaum in den Fokus der Aufmerksamkeit des Betrachters oder in diesem Fall des Lesers.

Betrachtet man beispielsweise ein Bild von Cézanne, dann sieht man zum Beispiel zunächst den berühmten Berg Mont St. Victoire. Im Falle einer solchen Malerei ist man sich jedoch auch bewusst, dass der Berg nicht als naturgetreue Abbildung erscheint, sondern durch den Maler stilisiert wurde: durch die spezielle Malweise Cézannes, seinen Farbauftrag, die Linienführung und die Eigenwilligkeit seiner Perspektive. Es kann sehr leicht geschehen, dass die Aufmerksamkeit des Betrachters vom Gegenstand des Bildes zu seiner malerischen Realisierung übergeht. Der Akt der Stilisierung kann dem Betrachter mehr oder weniger bewusst sein. Würde in einer Fernsehdokumentation eine Aufnahme des Berges gezeigt, so würde der Stil des Fernsehbildes kaum zum Gegenstand bewusster Betrachtung. Im Falle des Gemäldes von Cézanne ist es jedoch naheliegend, nicht nur den Gegenstand, sondern auch den Stil seiner malerischen Realisierung zu betrachten.

Dieses Phänomen geschieht beim Lesen eines Romans von Proust beispielsweise eher selten. Seine Beschreibungen in Swanns Welt ${ }^{6}$ werden die geistige und imaginative Aufmerksamkeit des Lesers so fesseln, dass die Buchstaben, die kleinsten sinnkonstitutiven Elemente des Textes, nicht als solche in den Blick rücken. Erst wenn der Leser ermüdet, nicht mehr dem Sinn und dem Gewebe des Textes zu folgen vermag, kann es dazu kommen, dass er plötzlich nur noch Buchstaben oder Worte sieht, ohne sie zu einem Sinnzusammenhang zusammenfassen zu können. Der Buchstabe verschwindet zwar nie ganz in der Lektüre, aufgrund seiner Unähnlichkeit zum Laut, dennoch ist er fast nie im Fokus der Aufmerksamkeit.

6 Marcel Proust: In Swanns Welt. Auf der Suche nach der verlorenen Zeit. Frankfurt a. M. 1997. 
Buchstaben sind in dieser Hinsicht ein besonders neutrales Medium. Sie sind als sinnkonstituierende Elemente immer klar erkennbar, die Materie der Schrift ist nie unsichtbar, dennoch drängt sie sich so gut wie nie gegenüber der Bedeutung in den Vordergrund; es sei denn man hält gerade eine besonders bibliophile Ausgabe eines Werkes in Händen, bei der es zuallererst auf die Kunstfertigkeit des Drucks ankommt. Dann werden die Buchstaben jedoch kaum noch als Buchstaben angesehen sondern als etwas bildähnliches, als besonderes schöne grafische Elemente.

Im Falle des Gemäldes von Cézanne sind es die Elemente des Bildes, die dazu führen, dass der Betrachter den Mont St. Victoire sehen kann. Der Stil ihrer Zusammenstellung beruht nicht oder kaum auf Konvention. Natürlich kommunizieren Malweisen mit anderen historischen und zeitgenössischen Stilen. Nur auf diese Weise kann ein Bild einen Bruch mit der Tradition darstellen, neuartig oder originell sein. Trotz dieses Anteils an Konventionalität muss jeder Maler (wenigstens wenn er als Künstler und nicht als Kunsthandwerker angesehen werden will) seinen Stil selbst finden. Gerade die Entwicklung eines individuellen Stils ist entscheidend für die Beurteilung von Bildern.

Der Buchstabe befindet sich gegenüber den Stilelementen von Bildern auf einer anderen Ebene. Seine Gestalt und die Anordnung in Gruppen zu bedeutungstragenden Einheiten ist nicht die Ebene der Kreativität, sondern die der Konvention. Die Zusammenstellung von Buchstaben zu Worten und Sätzen bildet den verhältnismäßig stabilen Teil des Sprachgeschehens. Diese Stabilität garantiert das Funktionieren der Sprache, die Lesbarkeit der Schrift, den gelingenden Austausch von Information. Kreativität im sprachlichen Ausdruck beginnt in den meisten Fällen erst auf der Ebene der Worte. Der Stil Prousts beispielsweise speist sich aus seiner Fähigkeit, Orte und Atmosphären zu beschreiben. Die Originalität des Schriftstellers hängt nicht davon ab, wie er mit Buchstaben umgehen kann, sondern von der Art und Weise, wie er Worte zu Sätzen fügt. Wesentlich seltener kommt es vor, dass Buchstaben und Laute in den Fokus der Aufmerksamkeit treten.

Einer der seltenen Fälle sind die Werke der konkreten Poesie und die davon inspirierten Gedichte Ernst Jandls. Hier tragen die Laute das Gedicht, sie entwickeln eine eigene Form von Sinn. Der Sinn dieser

7 Zum Übergang zwischen Buchstaben und Bildern, insbesondere im Graffiti siehe den Beitrag von Ralf Beuthan in diesem Band. 
poetischen Gebilde speist sich viel direkter aus der Buchstabenmaterie als es sonst der Fall ist. Jandls Gedichte stellen bei der Frage nach dem Wesen des Buchstaben ein interessantes Beispiel dar, weil hier Laut und Buchstabe zu expliziten Stilelementen werden (anders als im Roman, wo die Worte die Stilelemente bilden). Laut vorgelesen entwickeln Jandls Gedichte eine eigene Klanglichkeit, eine Melodie, die auf der Häufung oder Gegenüberstellung von Lauten beruht, die gerade in ihrer Lautlichkeit erhalten bleiben und nicht in der Bedeutung der Worte untergeht. Doch auch im Schriftbild erscheinen die Buchstaben in diesen Gedichten als selbstständige bedeutungstragende Einheiten. Die Anordnung der sprachlichen Zeichen in Jandls Gedichten (Worte oder auch nur Buchstaben) wird zum Stilmoment. Die physische Präsenz der Schriftzeichen tritt hier anders als in den meisten Romanen als zentrales Element auf. ${ }^{8}$ An dieser Form der Poesie wird deutlich, dass auch der gedruckte Buchstabe mehr ist als bloßes Zeichen für einen Laut, er wird durch sein Schriftbild selbst zum Bedeutungsträger.

\footnotetext{
8 Es ist interessant, dass diese Gedichte in einer Zeit entstehen, die von den Schriftstellern selbst als eine Krisenzeit der Sprache empfunden wird. Nach 1945 und damit nach dem Grauen von Auschwitz stellte sich für viele Intellektuelle die Frage, in welcher Form man nun noch Kunst schaffen oder Philosophie in angemessener Weise betreiben könne. Dies zeigt sich paradigmatisch in der vernunftkritischen Philosophie Theodor W. Adornos. Bezüglich der Kunst wurde die Forderung laut, sie dürfe nicht mehr schön sein, weil eine Ästhetisierung des Leidens moralisch nicht tragbar wäre. Die Frage ist dann, ob Kunst überhaupt noch möglich sei nach Auschwitz, schließlich kann sie nicht anders, als ästhetisch darzustellen. Für die Poesie stellte sich die Frage nach einer anderen, neuen, nicht ideologisch belasteten Sprache. Die Sprache, die das Medium der verheerenden Ideologie darstellte, konnte unmöglich das Medium einer neuen Poesie sein. Jandls Gedichte stehen nicht nur aber auch für diesen Versuch, eine neue Form sprachlichen Ausdrucks zu finden. In Anbetracht der Krise wählen Jandl und andere Künstler den Weg zurück zum Elementaren. Dieser Rückgang auf das Elementare begann in Kunst und Literatur jedoch schon zu Beginn des 20. Jahrhundert, man denke nur an die abstrakte Malerei, den Kubismus oder die Konzeptkunst. Der Rückgang auf elementare Formen scheint ein Merkmal des 20. Jahrhunderts zu sein. $\mathrm{Zu}$ diesen künstlerischen Formen des Elementaren gehört auch die Poesie der Laute und Buchstaben.
} 


\section{DER BUCHSTABE IN DER PHÄNOMENOLOGIE}

Edmund Husserl spricht im zweiten Band seiner Logischen Untersuchungen $(1901)^{9}$ in der Hauptsache von Zeichen, nicht von Buchstaben. Er unterscheidet zwei Formen dieses Begriffs: den Ausdruck und das Anzeichen. Das Anzeichen hat im Gegensatz zum Ausdruck keine Bedeutung (es kann aber muss keine Bedeutung haben, wenn es eine Bedeutung hat, dann kommt diese Funktion zu der ursprünglichen Funktion des Anzeigens hinzu). Unter die Klasse der Anzeichen fallen Gegenstände (wie Denkmäler, der Knopf im Taschentuch für die Erinnerung) oder Spuren. Diese Anzeichen verweisen auf eine (sei sie gegenwärtig, erwartbar oder vergangene) Präsenz dessen, was angezeigt wird. Die Vorstellung eines Verweisungszusammenhangs der auf eine ursprüngliche Präsenz abzielt, wird von Derrida aufgenommen und kritisiert.

Die sprachlichen Zeichen zählen laut Husserl zum Ausdruck, ${ }^{10} \mathrm{~d}$.h. zu den bedeutungstragenden Zeichen. Hierbei unterscheidet er zwischen dem Ausdruck seiner physischen Seite nach (Laute, Schriftzeichen auf Papier) und den psychischen Erlebnissen, die mit dem Ausdruck assoziiert sind. Das Anzeichen steht in dieser Theorie in einer zweistelligen Relation: Es ist immer Anzeichen von etwas. Demgegenüber ist mit dem Ausdruck im Kommunikationszusammenhang eine dreistellige Relation gedacht: Der Ausdruck hat eine „kundgebende Funktion“, ${ }^{11}$ indem er auf die seelischen Zustände und Intentionen des Sprechers verweist (in diesem Sinne ist er Anzeichen, den er verweist auf eine Präsenz). Gleichzeitig ist der Ausdruck bedeutsam: Der Inhalt, der kommuniziert werden soll, tritt als Sinnzusammenhang auf. Mit anderen Worten: Der Ausdruck verweist zugleich in Richtung des Innen (die Intentionen und Zustände des Sprechers) und in Richtung des Außen (das sinnlich Wahrnehmbarmachen dieser Intentionen in Form eines Sinnzusammenhangs).

9 Edmund Husserl: Gesammelte Schriften. Hrsg. von Elisabeth Schröter. Hamburg 1992. Bd. II: Logische Untersuchungen.

10 Ebd., S. 38 ff.

11 Ebd., S. 40. 
Wie weiter oben bereits angedeutet, ändert sich auch der intentionale Gehalt von Zeichen je nach Intention. Man kann ein gedrucktes Wort als physisches Vorkommnis betrachten, das Druckbild der einzelnen Buchstaben. Dann wird jenes jedoch nicht als bedeutsames, um mit Husserl zu sprechen, als Ausdruck, wahrgenommen. Legt man den Augenmerk auf die Bedeutung des Wortes oder Satzes, dann tritt diese Wahrnehmung des physisch realisierten Wortes nahezu vollkommen in den Hintergrund.

Dennoch ist die Physis ein nicht zu vernachlässigendes Charakteristikum des Buchstabenkonzepts. Die phonetische Schrift basiert auf Lauten, jedes Schriftzeichen repräsentiert einen bestimmten Laut. Maurice Merleau-Ponty beschreibt in seinem fragmentarischen Spätwerk Das Sichtbare und das Unsichtbare den Menschen als das "tönende Wesen“, das sich von innen her hört. ${ }^{12}$ Was hier in philosophischen Metaphern ausgedrückt werden soll, ist die Fundierung des Denkens und der Sprache in der Leiblichkeit und dem wahrnehmenden Bezug auf die Welt. Der Ursprung von Sprache als Medium der Reflexion und mithin der begrifflichen und ontologischen Unterscheidung von Subjekt und Objekt liegt in der der Lautproduktion eigenen Leiblichkeit. Diesen interessanten Gedanken lohnt es, ein Stück weit zu verfolgen.

Der Ausgangspunkt Merleau-Pontys ist die Welt der Wahrnehmung. Er untersucht die Eigenschaften von Wahrnehmung, weil er darin indirekt dem Sein des Logos, der Rationalität auf die Spur kommen will. Dass der Mensch sich als denkendes Wesen fundamental von der Welt als Gegenstand seines Erkennens unterscheidet, ist in dieser Theorie nicht der Ausgangspunkt, sondern das Rätsel, was es zu lösen gilt. Um dieses Rätsel zu lösen, beginnt Merleau-Ponty die Untersuchung in seinem frühen Hauptwerk, Die Phänomenologie der Wahrnehmung $(1945)^{13}$ beim Leib des Menschen: Weil wir leibliche, wahrnehmende Wesen sind, gibt es überhaupt erst Denken und Sprache. Sein Leib verbindet den Menschen mit den Dingen, nicht nur weil er die biologische Bedingung der Wahrnehmung ist, sondern weil der Mensch durch seine Leiblichkeit den wahrnehmbaren Dingen ähnelt. Durch den Leib sind wir nicht nur sehend, sondern auch sichtbar, nicht nur fühlend sondern auch fühlbar und vor allem konstituiert der Leib unsere Perspektive auf die Welt.

12 Ebd., S. 189.

13 Vgl. Maurice Merleau-Ponty: Phänomenologie der Wahrnehmung. Berlin 1966 (1945). 
Diese Perspektive ist jedoch nur eine in einem umfassenden Geflecht der Perspektiven des Sichtbaren - jenes Geflecht, das Merleau-Ponty später mit den Begriffen des Chiasmus und der Tiefe ${ }^{14}$ (der, und dies sei hier nur angemerkt nicht nur räumlich, sondern auch zeitlich verstanden wird) bezeichnen wird. Die Verbindung mit der Welt ist also zunächst eine symmetrische, auf Basis dessen was wir mit den Dingen teilen, nicht eine asymmetrische des einseitigen Erkennens und Feststellens der Dinge durch den erkennenden Geist.

Die Sprache ist für Merleau-Ponty ein besonderes Phänomen, weil hier aus einem leiblichen Verhalten heraus sich die Dimension des Sinns und der Transzendenz öffnet. Interessant ist das im Blick auf den Buchstaben vor allem deshalb, weil Merleau-Ponty diese Bewegung des Öffnens der Sinndimension nicht an das Denken bindet, sondern an das leibliche Verhalten der Lautproduktion:

Im Subjekt wie im Zeugen seines Verhaltens dezentriert sich plötzlich das zuvor bestimmte System der Vermögen, bricht auseinander und gestaltet sich neu nach einem Gesetz, das beiden unbekannt war und gerade in diesem Augenblick erst sich mitteilt. So wird z. B. das Runzeln der Stirn, nach Darwin ursprünglich zum Schutz der Augen vor der Sonne bestimmt, oder der konzentrierte Blick, eigentlich zum schärferen Sehen dienend, Bestandteil des menschlichen Ausdrucks beim Nachdenken und bedeutet dies dem Betrachter. Das Problem der Sprache ist ganz dasselbe: eine Kontraktion der Kehle, ein zischendes Entgleiten lassen der Luft zwischen Zunge und Zähnen, eine gewisse Spielart des Leibverhaltens erschließt sich plötzlich einem übertragenem Sinn und bedeutet ihn unserer Umwelt. ${ }^{15}$

Hier findet laut Merleau-Ponty das Wunder statt, das aus einem Verhalten eine Geste, aus einem Ton einen Ausdruck werden lässt. Denkt man an Husserls Unterscheidung zwischen Anzeichen und Ausdruck, findet hier der Übergang zwischen beiden statt. War das Runzeln der Stirn leibliche Reaktion auf die Präsenz der Sonne, ein Anzeichen für ihre Präsenz, so wird es nun zum Ausdruck der Innerlichkeit des

14 Vgl. Merleau-Ponty: Das Sichtbare und das Unsichtbare (wie Anm. 2). 15 Merleau-Ponty: Phänomenologie der Wahrnehmung (wie Anm.13), S. 229. 
Menschen, der Bewegung des Mentalen, das sich einen Weg bahnt in die physische Welt.

Merleau-Ponty bindet das Aufbrechen des Sinns, die Übergang leiblichen Verhaltens hin zum Ausdrucksgeschehen an die Produktion der Laute. In dieser Produktion findet sich nicht nur ein biologisches Faktum, das, wenn es nur auf hinreichend komplexer neuronaler Basis stattfindet, emergente mentale Gehalte zur Folge hat. Vielmehr sieht Merleau-Ponty in dieser elementaren Produktion von Lauten eine basale Form von Reflexivität, nämlich indem dies immer ein Sich-Selbst-Vernehmen beinhaltet. Diese Reversibilität von Hören und Hörbarmachen schließt an ein wiederkehrendes Merleau-Pontysches Thema an: das der Reversibilität und chiastischen Verschränkung zwischen Sehen und Sichtbaren, Sichtbaren und Unsichtbarem. Die ontologische Grundstruktur der Wahrnehmung, die Austauschbarkeit und Verschränkung von Perspektiven - Perspektiven, denen die Wahrnehmung immer schon eingeschrieben ist - bezeichnet Merleau-Ponty in Das Sichtbare und das Unsichtbare als Fleisch (la chair). Mit der Rede vom Fleisch ist nicht das tote, verzehrfertige Fleisch gemeint, sondern das des lebendigen Körpers, welches die Fähigkeit der Empfindung ermöglicht.

Das Fleisch als generalisierte Empfindbarkeit stellt die Grundstruktur der wahrnehmbaren Welt dar und figuriert zugleich als die Keimzelle des Logos, der Rationalität. Indem zum Beispiel zwischen Hören und Hörbarmachen eine Reversibilität vorhanden ist, beginnt bereits ein reflexiver Prozess der Unterscheidung zwischen Eigenem und dem Anderen, sowie der Aufnahme des Anderen in das Eigene - die Bewegung des Ausdrucks:

Wie es eine Reflexivität des Berührens, des Sehens und des Systems Berühren-Sehen gibt, so gibt es auch eine Reflexivität zwischen Vorgängen der Lauterzeugung und Gehör; jene schreiben sich klanglich ein, und jeder Stimmlaut weckt ein motorisches Echo in mir. Diese neuartige Reversibilität und das Auftauchen des Fleisches als Ausdruck verweisen auf die Einfügung des Sprechens und Denkens in die Welt des Schweigens. ${ }^{16}$

16 Merleau-Ponty: Das Sichtbare und das Unsichtbare (wie Anm. 2), 189. 
Der zentrale Gedanke Merleau-Pontys bezüglich der Sprache ist es, ihren Ursprung in den Leib zu verlagern, nämlich in die Lautproduktion. Geht man auf die Laute zurück, um das Aufbrechen des Sinns und des Ausdrucks in der Welt der Wahrnehmung zu beschreiben, dann geht man auf etwas Elementares zurück, dessen Abbild die Buchstaben sind. Die Buchstaben stehen ihrerseits für die Verkörperung der Sprache, für die Loslösung der Bedeutung vom Sprechakt. Auch den Buchstaben geht als Elementarform der Sprachzeichen ihr Bezug zur Körperlichkeit nicht verloren, denn sie schreiben sich in unsere Körper im Schreibund Leseprozess ein. Diesem Körper entstammen die Laute. Diese sind ihrerseits das Element gewesen, in dem der Ausdruck sich formieren konnte. Merleau-Pontys Sprachauffassung kann man zusammenfassend folgendermaßen charakterisieren: Er kritisiert die Idee einer reinen selbstgenügsamen Sprache und die eines von subjektiven Wahrnehmungseinsprengseln freien Logos, indem er Denken und Sprache an ein welthaltiges Wahrnehmungsgeschehen zurückbindet. In diesem fundamentalen Weltbezug wird jedoch keine reine Präsenz gedacht, vielmehr bricht das wahrnehmende Subjekt das Sein durch seine Perspektive auf und gibt so dem Nichtsein Raum. In diesem Wahrnehmungsgeschehen ist es die phoné, die Verlautbarmachung als leiblicher Prozess, die den Ausdruck ermöglicht und Sinn produziert.

Auch Derrida kritisiert die Idee eines reinen sprachlichen Sinns, er bindet diese Idee jedoch gerade an die abendländische Vorstellung des Logos als die sich selbst vernehmende Stimme. Er konstatiert einen Phonozentrismus in diesem Denken, der für die Selbstpräsenz, für einen reinen Sinn ohne Vermittlung steht. Dieser reine Sinn konstituiert sich in der, von der Vermittlung durch Schriftlichkeit reinen, in der Immanenz des denkenden Subjekts sich vollziehenden Sprache. Dieses Präsenzdenken, dass die Dimension des Sinns loslöst vom materiellem Geschehen und zu einer abgelösten Sphäre hypostasiert, birgt für Derrida den Keim von Gewalt und Ideologie. Demgegenüber skizziert er in der Grammatologie $(1967)^{17}$ ein Bild der Sprache, in dem Bedeutung gerade im Material, in der Schrift erst konstituiert wird. Um dies deutlich zu machen, gibt er dem Begriff der Differenz (différence) eine neue Bedeutungsdimension, indem er einen Buchstaben austauscht und von différance spricht. Damit macht

17 Jacques Derrida: Grammatologie. Übersetzt von Hans-Jörg Rheinberger und Hanns Zischler. Frankfurt a. M. 1974. 
er deutlich, wie im Gedanken einer prinzipiellen Wiederholbarkeit immer schon die Nichtidentität, die Fortschreibung von Differenz als sinnstiftenden Geschehen, angelegt ist. Hier ist nicht der Ort, die Derridasche Metaphysikkritik ausführlich zu behandeln. Daher in aller gebotenen Kürze der Kerngedanke zum Buchstaben: Derrida kritisiert die vor allem auf Ferdinand de Saussure zurückgehende Auffassung der geschriebenen Sprache als einfaches Abbild der gesprochenen Sprache, welche ihrerseits das Ideal nicht medial vermittelten reinen Sinns darstellt. Es gibt aber bedeutsame, sinnkonstitutive Unterschiede, die sich gerade nicht in der gesprochenen Sprache manifestieren, sondern die erst in der Schrift manifest werden, wie es zum Beispiel beim Begriff der différance der Fall ist. Die Schrift, die materiale Seite der Sprache wird damit zum Motor einer Sinnproduktion, die erst durch den Abstand vom reinen Sprechen möglich wird. Indem der Ausdruck des reinen Seelenlebens materialisiert wird, findet sich eine Ebene von Bedeutungen, die gerade nicht auf der Präsenz der Stimme basiert und damit der Rationalität eine Anderes, Materiales gegenüber stellt.

Sowohl die Schrift und ihre Elemente - die Buchstaben im Falle Derridas als auch die leibgebundene Lautproduktion Merleau-Pontys betonen eine elementare Dimension des Ausdrucks. Dieser Ausdruck findet nicht in einer abgeschlossenen Sphäre reiner Rationalität statt, sondern ist immer im Zusammenhang mit dem Anderen der Rationalität (oder ihrem zutiefst verwandten) zu denken: der Leiblichkeit und der Schrift. Gerade in diesem Bezug auf Elementares entsteht die Produktivität der Sprache, die Nicht-Abschließbarkeit des Ausdrucks. Damit ist der Buchstabe nicht nur ein Stellvertreter des Lautes und kontaminierendes Medium eines ursprünglich reinen Sinns, sondern Bedingung der Möglichkeit für die Fortschreibung des Denkens.

Beim Konzept des Buchstabens ist es naheliegend, Natur und Kultur einander gegenüberzustellen, die gesprochene Sprache als ursprünglich und natürlich zu begreifen, ihre Verschriftlichung jedoch als Künstlichkeit, als mediale Verklärung der ursprünglichen Sprache zu begreifen. Sowohl Merleau-Ponty als auch Derrida kritisieren auf ihre je eigene Art und Weise diese Gegenüberstellung. Für Merleau-Ponty bilden Natur und Kultur keine Gegensätze sondern eine Kontinuität. Für Derrida ist der Verweis auf eine der medialen Kultur vorgängige Natur des Sinns Zeichen einer überkommenen Form der Metaphysik, der Rückgang auf einen der Darstellung entzogenen Ursprung. Denkt man diese beiden sehr unterschiedlichen Positionen im Blick auf den Buchstaben weiter, dann wird deutlich, dass die Lautschrift nicht einfach ein Abbild der 
Sprache darstellt. Mit dem Buchstaben eröffnet sich die Möglichkeit von Sinnproduktion, die nicht als der Natur gegenübergestellte Sphäre begriffen werden muss. ${ }^{18}$

18 An dieser Stelle sei noch auf die Theorie des Kognitionspsychologen Merlin Donald verwiesen. Er begreift das Entstehen der Schrift als einen folgenreichen Schritt. Indem wir Bedeutung außerhalb unseres individuellen Geistes bewahren und zugänglich machen können, haben wir unseren Geist gewissermaßen veräußerlicht. Diese Veräußerlichung oder Verkörperlichung des Mentalen wirkt aber erstaunlicherweise auf unsere kognitiven Fähigkeiten zurück. Diese können wir laut Donald erst im sozialen Verbund und durch die Nutzung äußerer Wissensspeicher wirklich ausprägen.Der Mensch ist eine kollektive Intelligenzform und die Buchstaben sind das sehr flexible Wundermittel zur Wahrung und Fortschreibung des Denkens und Verstehens. Die Buchstabenschrift bietet dabei die Möglichkeit, Sinn immer neu zu modulieren, auf ihrer Basis wird er nicht nur festgeschrieben sondern vor allem auch dynamisch und damit zum Gegenstand einer Evolution kultureller Art, die der natürlichen nicht so unähnlich ist, wie man glaubt. Vgl. Merlin Donald: A Mind so Rare. New York 2002. 



\section{VOM GEIST DER BUCHSTABEN}

\section{Medienphilosophische Überlegungen im Anschluss an Hegel}

I.

„Die Grenzen meiner Sprache bedeuten die Grenze meiner Welt“ ${ }^{\text {}}$ - Wittgensteins berühmtes Diktum ist ein frühes Dokument, ja gewissermaßen ein Gründungssatz sprachanalytischer Philosophie. In ihm klingt - trotz mancher Umbrüche - zugleich ein spätes Erbe romantischer Philosophie nach, welche einst gegen den Kantischen Rationalismus das Denken aus dem starren Gefüge seines kategorialen Systems heraus- und in das offene und fruchtbare Feld der Sprache hineinführen wollte. Die sich im Kontext der Romantik bahnbrechende Einsicht lautete: ,Denken ist Sprache'. Dabei wird die Sprache ihrer unendlichen Produktivität theoretisch entdeckt und ästhetisch erforscht. Doch während sich die Romantiker noch geradezu an den Früchten ihrer neuen Einsicht berauschten, wird ein gutes Jahrhundert später Wittgenstein vollkommen ausgenüchtert die logisch-epistemologischen Konsequenzen aus jener These ziehen und dabei - das zeigt jenes Diktum - das Feld der Sprache auf seine Grenzen hin sondieren. Aber noch Wittgensteins Sprachreflexion ist freilich mehr als eine durch allerlei operative Termini aufgerüstete Sprachbeschreibung. Es geht hier um nicht weniger als um die Welt und unser Wissen von der Welt. - Wie aber stehen wir zu diesem Erbe, heute, etwa zwei Jahrhunderte nach den Romantikern und ein knappes Jahrhundert nach

1 Ludwig Wittgenstein: Werkausgabe in 8 Bänden. Frankfurt a. M. 1984. Bd. I.: Tractatus logico-philosophicus (1921), 5.6. 
Wittgensteins nüchterner Analyse, wonach unser Wissen von Welt in den Grenzen der Sprache befangen ist? Mir scheint, dass wir heute - irgendwie noch immer von unserer Sprachfähigkeit begeistert - vielleicht mehr denn je versuchen, den Bedingungen, und d.h. auch den Grenzen der Sprache auf die Schliche zu kommen, um uns selbst in unserem Weltverhältnis aufzuklären.

Ich werde im Folgenden nur ein einziges, nämlich das kleinste und stillste Element der Sprache fokussieren - den Buchstaben. Es wird darum gehen zu überlegen, welche Rolle Buchstaben im angedeuteten Zusammenhang spielen, $d . h$. in einer Theorie- und Denktradition, in der Sprachtheorie nur verbunden mit Fragen nach unserem Weltverhältnis entwickelt wird. Meine These ist, dass Buchstaben Markierungen einer basalen Grenze der Sprache sind; sie sind, salopp gesagt, ein Grenzphänomen der Sprache. Ich will in einer freilich nur idealtypischen Weise, vier verschiedene Optionen konturieren, wie man mit diesem Grenzphänomen theoretisch umgehen kann bzw. umgegangen ist. Anhand eines Beispiels - einem Graffito - soll ein Strukturzusammenhang verdeutlicht werden, der jedoch nur von einem der vier Optionen theoretisch fassbar wird. Eine solche, meines Erachtens wegweisende, Theorieoption ist, wie ich zu zeigen versuche, vom Hegelschen Ansatz aus zu begründen.

II.

Vorüberlegung zur Idee eines ,Grenzphänomens'. - Ein Phänomen, was innerhalb eines bestimmten Erklärungs- und Verständnishorizontes als gänzlich fremdartig erscheint, also scheinbar nicht in diesen Horizont gehört, aber dennoch - wie aus einer anderen Ordnung oder Dimension - in diesen Horizont einbricht, markiert eine Grenze des jeweiligen Horizontes; es ist gewissermaßen ein Zeichen für etwas, das einem Außerhalb des Horizontes angehört, und doch innerhalb des Horizontes erscheint, ohne den Regeln zu folgen, die diesen Horizont eröffnet haben. Das Zeichen eines Jenseits der diesseitigen Ordnung ist ein Zeichen ihrer Grenze. Es erscheint hier - und gehört hier nicht her. Es ist ein Grenzphänomen, das die Erklärungskraft eines Horizontes zur Disposition stellt. - Doch was im Theoriegeschäft Alltag sein mag und durch Routine und institutionelle Sicherheitssysteme in der Regel gebändigt wird, hat dennoch einen existenziellen Kern. Denn Grenzphänomene haben per se etwas Beunruhigendes und Unheimliches. Und einige Denker der Philosophie des 19. und 20. Jahrhunderts haben - fasziniert von den Unheimlichkeiten, 
denen sich ein menschliches Leben ausgesetzt findet - gleichsam den Urtypus jener Unruhe, die durch eine mein Ordnungssystem erschütternde Grenzerfahrung ausgelöst wird, auf den Begriff zu bringen gesucht - und sie haben dafür den Begriff der ,Angst' geprägt. ${ }^{2}$

Nun mag es übertrieben erscheinen, die Begegnung mit Buchstaben mit dem Begriff der ,Angst ${ }^{\star}$ in Verbindung zu bringen. Aber schauen wir genauer hin: Buchstaben markieren einen prekären Punkt in der Sprache. Sie verweisen nur auf sprachliche Sinneinheiten, und durch eine regelgeleitete Kombinatorik lassen sich auch Sinneinheiten generieren - aber sie erscheinen nicht an ihnen selbst als sinnhaft. Buchstaben sind nicht nur als bloße Zeichen für Laute, sondern auch in semantischer Hinsicht stumm. Sie verweisen auf mögliche Sinneinheiten, mahnen gleichsam Bedeutung an, sind aber an ihnen selbst bedeutungsleer. Buchstaben sind unmittelbar nichts anderes als stumme Mahnzeichen - Monster, deren beunruhigende semantische Nichtigkeit eine von ihnen selbst her unerschließbare Semantik anzeigt.

Wenn man Sprache als ein Spiel von Sinn und Unsinn unter der Führung des Sinns auffasst, dann sind Buchstaben jene kleinen Instanzen des Unsinns, die in diesem Spiel mitspielen und ständig von den Grenzen des Sinngeschehen zeugen, dessen Mitspieler sie nolens volens sind. Sie sind die kleinsten und elementarsten Instanzen. Kleinere Zeichenstrukturen, visuelle Distinktionen ,unterhalb' der Buchstabentypik würden wir nicht mehr als sprachliche Zeichen, allenfalls noch als eine Art visuelles Rauschen - wie die Pixel eines defekten Fernsehers - wahrnehmen. Größere und komplexere Zeichenstrukturen führen dagegen ,in der Regel' bereits zu identifizierbaren Sinneinheiten (z. B. Worte), sind also bereits semantisch zugänglich, ,verständlich‘. Letztere machen sich gleichsam ,durchsichtig', lassen ihre opaken und bedeutungsleeren Mitspieler - die kleinen Buchstabenmonster - in der Transparenz eines Bedeutungszusammenhangs verschwinden. Einmal durch jene kleinen Monster angestoßen in die semantische Ordnung eingetaucht, übernimmt die Vorstellung einer durchgängigen Sinnhaftigkeit die Führung, und aus dem Feld der Sprache scheint ein System von ungebrochener Bedeutungstätigkeit zu emergieren. In ihm herrscht die Logik inferentieller Verhältnisse. Als Mitspieler dieses Systems wissen wir immer mehr als das semantische Nichts unmittelbarer

2 Vgl. Sören Aabye Kierkegaard: Der Begriff der Angst (1844). Philosophische Bissen. Die Krankheit zum Tode. Hamburg 2005; Martin Heidegger: Sein und Zeit (1927). Tübingen 2006. 
Gegebenheiten; sowie wir in das System von Bedeutung eintreten, steht alles und jedes in Vermittlungs- und Folgerungsverhältnissen. Wissen wir jenes, dann können wir auch auf dieses schließen usw. Doch wenn wir nicht in das System eintreten, indem wir etwa gebannt auf jene Monster starren, dann verschließt sich das Bedeutungssystem. Denn aus Buchstaben lässt sich nichts schließen. Buchstaben zeigen eine Ordnung an, beweisen aber gar nichts.

Ich möchte das, worauf ich hier zunächst hinauswill, mit einer terminologischen Unterscheidung verdeutlichen: Insofern Buchstaben bedeutungslose Elemente sind, die Bedeutung nur anzeigen, welche erst auf einer höheren Ebene generiert wird, sind es Elemente, die gewissermaßen Bedeutung nur monstrieren [abgeleitet aus lateinisch monstrare]. Schaute man von ihnen aus mal nicht in den semantischen Sprachraum hinein, sondern versuchte durch sie aus dem semantischen Raum herauszuschauen, dann blickte man gleichsam in die Bedeutung suggerierende Maske erschreckender Bedeutungslosigkeit. Folgt man hingegen den Regeln des semantischen Raums, d. h. bewegt man sich ,ordnungsgemäß innerhalb der Sprache, ohne sich von ihren bedeutungslosen kleinen Monstern ins Stolpern bringen zu lassen, dann kann man sich zu jenen logischen Bewegungen aufschwingen, die wohl im Zentrum der Sprache, aber eben nicht an ihren spröden Buchstabenrändern möglich sind: Es sind Bewegungen, die nicht monstrieren, sondern die etwas demonstrieren, Bewegungen, die sich zu durch semantische Relationen vermittelten Beweis- und Begründungsgängen ausformen. Während also Buchstaben als stumme Mahnmale - an ihnen selbst bedeutungsleer - zeigen bzw. monstrieren, d.h. einen noch nicht bestimmten, opaken semantischen Raum anzeigen, beginnt der bedeutungsschwangere Sprachraum scheinbar erst dort, wo etwas als etwas artikuliert wird und so als Instanz einer Folgerungsbeziehung Verwendung finden kann. - Um es also kurz zu machen: Buchstaben sind die kleinen Monster der Sprache, durch die der Wahnsinn in die sich stets ankündigende Sinnfülle der Sprache einzubrechen vermag. Es sei nur an die vielfältig variierende Erfahrung erinnert, in der man des Lesens und Schreibens müde unverwandt auf einen Haufen von Buchstaben stiert, welche förmlich aus einer gähnenden Leere auf einen zurückschauen - der horror vacui der Schreibenden und der frustrierten Leser. 
III.

Der Horror, der von solcherlei Monstern ausgehen kann, mag in unserem alltäglichen Leben und Umgang mit Buchstaben zu vernachlässigen sein - er ist aber doch beträchtlich genug, um aus ihm theoretisches Kapital zu schlagen. Wenn Monster auftreten, schlägt die Stunde heldenhafter Gegenangriffe. Die Geschichte des polemischen Verhältnisses zwischen den Buchstaben und einer lebendigen Sinnfülle ist durch solche Heldenauftritte getaktet. Sie ist ebenso vielgestaltig wie lang, von geradezu biblischem Alter. Einer ihrer Helden war und ist Fichte, der gegen die tote, nur mechanisch zusammengehaltenen und ebenso nur mechanisch, „mit saurer Mühe“3 dechiffrierbaren „Buchstaben“ eine „belebende Kraft"4 beschwor. Er nannte dieses Gegenprinzip, das uns vor der ,dumpfen‘, gar ,tödlichen' Buchstäblichkeit schützen sollte: ,Geist' - und spielte damit auf nicht weniger als auf jenes berühmte Paulus-Wort an: „der Buchstabe tötet, aber der Geist macht lebendig“ (2. Korinther, 3,6). - Ich darf hier jedoch den religiösen Kontext ausklammern. Entscheidend ist für meine Überlegungen nur, dass es eine lange Tradition gibt, in der Buchstaben in einem polemischen Verhältnis zu einem lebendigen und sinnproduzierenden Prinzip, dem ,Geist' gedacht werden. - Wie aber lässt sich aus der Opposition von toten Buchstaben und lebendigem Geist theoretisches Kapital schlagen? Die Antwort liegt, wie bei den meisten Streitfällen, nahe: indem man sich entweder auf eine Seite schlägt, um diese dann im Kontrast zur anderen Seite glänzen zu lassen; oder indem man einen integrativen Ansatz entwickelt, der beide Seiten als einen funktionalen Zusammenhang erkennen lässt, auf dem ein friedvolles Verhältnis gedeihen sollte [was aber, wie Lyotard mit seinem Konzept des ,Widerstreits ${ }^{65}$ beispielhaft herausgearbeitet hat, das Problem einer Funktionalisierung zugunsten einer Seite birgt].

Welche Theorienansätze oder Kernthesen ergeben sich aus der genannten Problemlage? Welche Strategien sind möglich?

3 Johann Gottlieb Fichte: Werke. Hrsg. von Immanuel Hermann Fichte. Nachdruck der Ausgaben Berlin 1845/46 und Bonn 1834/35. Berlin 1971. Bd. VIII.: Über Geist und Buchstab in der Philosophie (1794), S. 270.

4 Ebd., S. 274.

5 Vgl. François Lyotard: Der Widerstreit (1983). München 1989. 
(1) Die erste Kernthese ist mit Fichte schon beiläufig angedeutet worden, der das polemische Verhältnis zwischen Buchstaben und Geist ganz im Sinne der paulinischen Tradition auflöst. Sein Ansatz folgt dem Motto: ,Geist statt Buchstaben'. Die dabei leitende Idee resultiert aus der Transformation der Kantischen Transzendentalphilosophie. Im Ausgang von Kants transzendentalem Ich konzipiert Fichte Subjektivität - das unvorgreifliche Selbstverhältnis des Ich zu sich selbst - als Quellpunkt und Strukturprinzip der Welt. Die These eines buchstabenfeindlichen Geistprimats wird argumentativ gestützt durch den Nachweis eines allein aus der Reflexivität des Ich produzierten semantischen Systems (= Welt). Die Mechanik einer Buchstabenkombinatorik erscheint hier als ein die eigentliche Produktivität des Ich und die darin begründete Syntax der Welt verstellender Schein. Die Strategie ist grosso modo die: Destruktion der Buchstabenmonster durch Einsicht in das Prinzip, dem ,schaffenden Geist $^{6}$. Die Buchstaben werden bei Fichte gleichsam,wegdemonstriert ${ }^{t}$, d. h. ausgelöscht im Licht eines reflexiv gesteuerten Demonstrationsgangs.

(2) Eine andere Strategie wird möglich, wenn das produktive Prinzip anders konzipiert wird. Setzt man an die Stelle eines ,absoluten Ich', welches sich per se der Traditionszusammenhänge und gattungsgeschichtlich überkommenen Vorgaben entledigt hat, ein anderes allgemeines Prinzip, nämlich die ,Sprache', dann wird ein ganz anderes Verhältnis zwischen Geist und Buchstabe denkbar. Mit Sprache bekommen Buchstaben allererst einen - wenn auch noch marginalen - Platz innerhalb der Prinzipiensphäre. Sie haben vielleicht noch kein volles Bürgerrecht, aber sie wohnen bereits legal an den Stadtgrenzen, da sie unentbehrliche Funktionen übernehmen. Denn anders als das ,absolute Ich' kann sich ,Sprache' per se nicht der Traditionszusammenhänge entledigen. Und anders als jenes soll Sprache dies auch nicht, da durch sie ja allererst vergangenes Wissen und überkommene Aufgaben weitergegeben werden - ein Übermittlungsprozess, welcher den vormaligen Buchstabenmonstern ein gewisses Recht verleiht. Die Bedeutung der Buchstaben für den Sprachprozess wird vor allem dort noch zunehmen, wo sie nicht nur für die Reproduktion, sondern auch für die Produktion von Bedeutung entdeckt werden. - Eine solche Position, die die Produktivität jener fichteschen Subjektivität innerhalb eines Konzepts von Sprache reformuliert, möchte ich als eine romantische Position bezeichnen. Das damit aber nicht einfach ,alter Wein in neuen Schläuchen' angeboten wird, ist spätestens dann klar, wenn wir

6 Vgl. Fichte: Werke, Bd. VIII (wie Anm. 3), S. 290. 
auf das Verhältnis von Geist und Buchstabe achten. Das Motto, unter das ich die romantische Position stellen möchte, lautet nicht mehr ,Geist statt Buchstaben', sondern: ,Geist durch Buchstaben'. Für die romantische Position ist entscheidend, dass der Geist (i) entschieden als Sprache und (ii) Buchstaben als produktives Moment der Sprache gedacht werden.

(3) Mit der romantischen Position wird zugleich der Weg frei für eine weitaus radikalere, ganz und gar anti-paulinische Strategie. Forciert man die Idee, dass Buchstaben produktive Momente der Sprache sind, dahin, dass allein der Mechanismus der Buchstaben produktiv ist, dann denkt man die Welt neu und anders. Die Welt ist gleichsam ,geistlos' geworden und soll es auch sein. Die Einhüllung der bloßen Faktizität der Welt durch eine sie umschließende semantische Sphäre, die Welt als ein bedeutungsvolles Ganzes, erscheint nun als kontingenter Effekt eines basalen Mechanismus, der eben nicht selbst Geist ist. Das paulinischfichtesche Grundverhältnis wird hier geradezu umgekehrt: Der Geist ist nun selbst ein die produktive Basis verstellender Schein; er ist nur ein Sekundäres - das Primäre sind die vormaligen Monster. - Es sei hier eine historische Randbemerkung erlaubt: Dass nach Fichtes, ursprünglicher Einsicht' das 19. und 20. Jahrhundert ganz unfichtesch eine Zeit der Monstergeschichten (Frankenstein, Golem, Dracula etc.) wurde, erscheint durchaus nicht als kontingent. Im Kontext dieser medienphilosophisch angelegten Perspektive wird überdies deutlich, dass die oft diagnostizierte ,Entzauberung der Welt in der Moderne ${ }^{67}$ nicht allein durch den Aufstieg der Naturwissenschaften im wissenschaftlichen Ranking der modernen Gesellschaft begründet ist. Dass z. B. McDowell nur diese Linie thematisiert, dürfte mit dem szientifischen Erbe der analytischen Philosophie, insbesondere mit seinem Sellarserbe zu tun haben. Dass aber die ,Entzauberung der Welt' vielmehr eine im Kern philosophische Angelegenheit war und ist, weil die Philosophie selbst sie vollzogen hat, und nicht erst, weil andere Disziplinen sie vor vollendete Tatsachen gestellt hätten, die es nun philosophisch einzuordnen gelte - dies bleibt merkwürdig ausgeblendet. - Doch welche weitere Strategie ist denkbar, um das polemische Geist/Buchstaben-Verhältnis für die Konstitution einer Theorieposition zu nutzen? Indem nicht die lebendige Sprache, sondern gar die Buchstaben, näher: der Mechanismus der Buchstabensetzung und -kombination die Prinzipienstelle einnehmen, werden wir

7 Vgl. John McDowell: Mind and World. With a New Introduction. Cambridge u. a. 1996. 
zum Antipoden der Fichteschen Position geführt. Motto und Grundthese lauten nun: ,Buchstaben statt Geist'. Und während Fichte noch auf die Auslöschung der Buchstaben in der Transparenz der Semantik zielte, avisiert diese Position die Austreibung des Geistes aus einer autorfreien Zone von algorithmisch beschreibbaren Automatismen. ${ }^{8}$ Der Rat bei der Begegnung mit Monstern wäre dann sicher nicht mehr: ,Rettet Euch ins Licht und vernichtet die dunklen Monster durch Lichtgeschosse'; sondern eher ein achselzuckendes: ,Das Leben wird überschätzt'.

(4) Es liegt auf der Hand, dass hier noch eine Theorieoption fehlt. Auf die Situation eines polemischen Verhältnisses zwischen Buchstaben und Geist reagierten, wie gesehen, zwei Theorieansätze so, dass sie sich jeweils auf eine Seite schlugen, und dann gegen die andere Seite eine Art Exorzismus praktizierten. Eine erste alternative Theorieoption, die ohne Exorzismus auskommt, bestand darin, die positive Funktion der Monster für die Produktion des Geistes herauszustellen. Die vierte und letzte, nicht weniger radikale Theorieoption wird darin bestehen, dafür zu argumentieren, dass die Monster selbst ein Produkt des Geistes sind. Um diese Theorieoption soll es abschließend gehen. Halten wir zunächst der Übersicht halber das nun maßgebende strategische Motto fest. Die Kernthese wird lauten: ,Buchstaben durch Geist . Einen Exorzismus braucht es hier nicht mehr. Die Sache richtig sehen reicht.

IV.

Ein Streitfall. - Die Begegnung mit Buchstabenmonstern, jenen semantisch stummen Grenzphänomenen der Sprache, ist heute im urbanen Raum eine ganz alltägliche Erfahrung. Es bedarf keineswegs erst der Müdigkeit von Schreibern und Lesern. Man muss einfach nur die öffentlichen Räume passieren und wird unweigerlich auf sie stoßen. Die Rede ist von Graffiti - eine Unmenge von erratischen im öffentlichen Raum zerstreuten Buchstabenzeichen, oft nicht dechiffrierbar, gelegentlich zu wortsuggerierenden Buchstabenensembles formiert.

Es ist da vorderhand durchaus plausibel, wenn einer der ersten Graffititheoretiker, nämlich Jean Baudrillard, ihre provozierende Bedeutungsund Sinnlosigkeit ins Zentrum seiner Überlegungen stellt. ${ }^{9}$ Entscheidend

8 Vgl. Friedrich Kittler: Austreibung des Geistes aus den Geisteswissenschaften. Programme des Poststrukturalismus. Paderborn u. a. 1980.

9 Vgl. Jean Baudrillard: Kool Killer oder: Der Aufstand der Zeichen. Berlin 1978 . 
für uns: Auch Baudrillard folgt im Kern der eben skizzierten Grenzlinie zwischen Geist und Buchstaben, akzentuiert die spezifische Monstrosität der Buchstaben, insofern sie qua Graffiti die allgemeine Sinnproduktion zu unterbrechen bzw. zu blockieren scheinen, statt sie zu befördern. Graffiti, das sind für Baudrillard zuletzt geistlose Buchstaben. Dass er dem semantischen Nichts eine politische Funktion zuschreibt, braucht hier nur erwähnt werden..$^{10}$ Wichtig ist in hier nur, dass er Graffiti primär als Buchstaben begreift und so die These ihrer Bedeutungslosigkeit stützt.

Doch gerade Graffiti lassen ein Merkmal von Buchstaben erkennen, das eine für Baudrillard wesentliche und vor mir im Vorangegangenen ebenfalls bemühte Prämisse in Frage stellt.

Zahllose Beispiele zeigen auch bei einem flüchtigen Blick einen ganz einfachen, aber theoretisch kaum beachteten Punkt: viele Graffiti kaprizieren sich auf die Bildlichkeit der Buchstaben. Man beachte nur die bildspezifischen Darstellungsmittel wie Farbigkeit, Umrisslinien, Perspektivität, graphische Dynamisierung der Fläche und Richtungssinn. Es mag also zutreffen, dass die Buchstaben als Elemente einer sprachlichen Ordnung als sinnlos erscheinen, aber als Elemente einer Ordnung des Bildes sind sie deswegen noch keineswegs sinnlos. - Um es also kurz zu machen: Was zahllose Graffiti - weltweit - zeigen, ist, dass Buchstaben sich dem sprachlichen Sinn entziehen können, ohne sich damit dem Sinn überhaupt entziehen zu müssen. Buchstaben bleiben Elemente einer visuellen Ordnung. Sie werden von Graffiti-Sprayern zumeist als Mittel polemischer Attitüden gegen einen diskreditierten gesellschaftlichen ,Geist' genutzt, aber sie fungieren auch als Mittel ästhetisch-experimenteller Erkundung einer zugleich bildlichen Sinnsphäre, deren Wurzel tiefer reichen könnte, als die einseitig am Sprachsinn orientierte Auffassung zugeben mag.

Folgt man dieser Spur, dass Buchstaben - und nicht erst Hieroglyphen - Momente der Bildlichkeit enthalten, dann wird man auch einen anderen, einen integrativen Theorieansatz suchen. Er müsste zweierlei leisten: (1) erklären, dass und wie die Buchstaben nur in einer einseitigen und eindimensionalen Betrachtungsweise als Monster erscheinen, und (2) erklären, in welcher Hinsicht sie ihre Monstrosität verlieren, ohne ausgelöscht werden zu müssen. Gefordert ist also ein Theorieansatz, der mehr als eine Ordnung des Sinns zu begreifen vermag und so ein Verständnis der Übergänge zwischen diesen Ordnungen erlaubt.

10 Dazu vgl. meinen Aufsatz: Was ist Graffiti? Versuch einer philosophischen Bestimmung. In: Ralf Beuthan, Pierre Smolarski (Hrsg.): Was ist Graffiti? Würzburg 2011, S. 121-139. 
V.

Wenn man - mit Fichte gesprochen - nicht auf den ,Buchstaben', sondern auf den ,Geist' der Theorie schaut, dann ist mit der Hegelschen Philosophie genau eine solche integrative Theorie gefunden. Man darf sich bei Hegel nur nicht von den dem jeweiligen methodisch-architektonischen Zusammenhang geschuldeten begrifflichen Distinktionen und Pointierungen ins Bockshorn jagen lassen. Es ist sicher richtig, dass Hegel für den Vorrang der Buchstaben qua Lautzeichen gegenüber den bildhaften Hieroglyphen argumentiert. Und man wird mit Derrida manchen Hinweis auf einen Phonozentrismus ${ }^{11}$ finden. Zweifellos. Aber damit wäre nur ein Punkt aus einer hochkomplexen und umfassenden Sprachtheorie herausgegriffen. Weitaus interessanter als die oft monierten Pointierungen ist jedoch die Mehrdimensionalität seiner Sprachtheorie, die es eben erlaubt, mehr als eine Dimension zu sehen - und das bedeutet hier: Monster nicht als Zeichen des Wahnsinns, sondern als Zeichen eines anderen Sinns erkennen lernen.

Zwei interessante Aspekte seien hervorgehoben. Es müsste erklärt werden, (1) dass Übergänge zwischen den Sinndimensionen möglich sind und (2) dass es eine Art Tiefendimension der Sprache gibt. Letztere wird vor allem von jenen Theorieansätzen ausgeblendet, die die Opposition von ,Buchstaben' und ,Geist' zugunsten einer Seite forcieren. Anders Hegel. Sein integrativer Theorieansatz kann unter das zuletzt genannte Motto gestellt werden: ,Buchstaben durch Geist'.

Was gewinnt man bei der Frage nach dem Verhältnis von Geist und Buchstaben, wenn man es mit Hegel betrachtet?

(1) Hegel argumentiert, wie gesagt, für einen Primat der Buchstaben gegenüber den Hieroglyphen, und zwar deshalb, um am Ende zu einem Konzept des Denkens kommen zu können, das den romantischen Primat der Einbildungskraft überwunden hat und das Denken aus der Bildhaftigkeit herausführt. Die Stoßrichtung seiner Überlegungen ist zusammengefasst die: Jegliche Bildhaftigkeit bleibt in der Figur von Repräsentation befangen und verstellt damit die Idee einer Objektivität, in der die Gedanken nicht nur Abbilder der Sache, sondern die Sache selbst sind. - Die

11 Vgl. Jacques Derrida: Grammatologie (1967). Übersetzt von Hans-Jörg Rheinberger und Hanns Zischler. Frankfurt a. M. 1974. 
damit verbundende Tendenz zu einem Phonozentrismus hindert Hegel jedoch nicht daran, die konkreten praktischen Vollzüge zu bedenken, in denen Bedeutung mittels Buchstaben generiert wird. Hegels Sinn dafür, dass die theoretischen und praktischen Vollzüge immer auch konkrete Tätigkeiten sind, d. h. zutiefst verwachsen sind mit verschiedenen anderen, oft unbemerkten und stillschweigend vorausgesetzten Tätigkeiten und Lebensvollzügen, wird oft vergessen. Achtet man mit Hegel nicht nur auf die distinkten und nicht-abbildenden Buchstaben, sondern vielmehr auch auf die konkreten Vollzüge und Gewohnheiten, in denen Buchstaben allererst eine sprachliche Funktion bekommen, dann wird man auch folgendes entdecken und sagen können: Obwohl Buchstaben als Zeichen von Lauten keine Hieroglyphen sind und das Hören eines Tons dem Sehen eines Bildes den Rang abgelaufen hat, wird in der Praxis des Lesens zugleich der Primat des Hörens wieder relativiert. Nur Anfänger lesen so, als ob sie etwas laut aussprechen. Fortgeschrittene Leser nehmen, so Hegel, nicht mehr einen „Umweg durch die Hörbarkeit“"12 und verwandeln „im Interesse des Sehens ${ }^{\text {“13 }}$ die „Buchstabenschrift in Hieroglyphen“"14. - Worauf es hier ankommt, ist folgendes: Hegel zeigt, dass Buchstaben ihre Funktion nur innerhalb einer allgemeinen, regelgeleiteten und durch Übung und Gewohnheit zur - wie man heute mit Hegel gerne sagt ,zweiten Natur' gewordenen Praxis entfalten können. Buchstaben sind also erst Buchstaben in und durch eine Praxis. Kurz: Buchstaben sind durch den Geist das, was sie sind. - Und da sie Elemente einer konkreten und vielschichtigen Praxis sind, ist es kein Einwand, sondern nur konsequent festzustellen, dass Buchstaben auch als Hieroglyphen oder Bildzeichen (und auch umgekehrt) fungieren können. Ein und dasselbe Element, z. B. das große , $\mathrm{A}^{\boldsymbol{}}$ könnte also prinzipiell als Zeichen eines Lautes oder als Hinweis auf das erste Element einer Zeichenreihe oder als Symbol einer pyramidalen Struktur verwendet werden. Doch weniger diese Vieldeutigkeit des Zeichens ist bemerkenswert, als vielmehr der Gedanke, dass die konkrete Sprachpraxis nicht in die Krise geraten muss, wenn die Buchstaben aus der orthographischen und grammatischen Regelhaftigkeit herausfallen. Durch die theoretische Einbindung der Praxis werden auch Übergänge, Transformationen denkbar zwischen verschiedenen Sinnordnungen, wie z.B. zwischen Bildlichkeit und Sprache. Und das,

12 Georg Wilhelm Friedrich Hegel: Werke. Frankfurt a.M. 1986. Bd. 10: Enzyklopädie der philosophischen Wissenschaften III (1830), S. $276 \mathrm{f}$.

13 Ebd., S. 276.

14 Ebd., S. 277. 
was als Buchstabenmonster erscheinen mag, ist dann vielmehr nur ein Übergang zwischen einer und einer anderen Sinnordnung und noch lange nicht das Ende der Welt, noch nicht einmal der Sprachwelt.

(2) Wenn die Monstrosität der Buchstaben daher rührt, dass sie eine Grenze der Sprache markieren, so ist mit Hegel, wie angedeutet, sowohl ihre Monstrosität erklärbar als auch ihre Harmlosigkeit: Sie fungieren als Elemente einer sprachlichen Ordnung und sind zugleich als Elemente einer transmedialen Praxis der Sinnproduktion Grenze und Übergang zu einer nicht per se sprachlichen Ordnung, z. B. der Bildlichkeit. - Doch mit Hegel kann man noch einen Schritt weiter, oder besser: tiefer gehen. Dass die Sprache verschiedene Verbindungen zur Visualität und Bildlichkeit pflegt, mag noch hingehen - aber ist nicht sowohl die visuelle als auch die sprachliche Ordnung noch dadurch gekennzeichnet, dass mit ihnen eine zuletzt körperlose Geistigkeit in Anspruch genommen wird? Und stehen dagegen nicht gerade Graffiti für eine Art von Buchstaben, die vor allem eine körperliche Geste, eine ,Handschrift' zum Ausdruck bringen, statt einen nur à corps perdu zugänglichen Sinn? Widerspricht also nicht Sinn und Sinnlichkeit der Graffiti der Hegelschen Geist- und Sprachkonzeption? - Ich bin in der Tat davon überzeugt, dass Graffiti - zumindest ein bestimmter Typus von Graffiti - Ausdruck eines Sinngeschehens sind, der nur durch seine Verwurzelung in der Leiblichkeit, den leiblichen Gesten des Einritzens, des schwungvollen Ziehens von rhythmischen Bögen etc. zugänglich ist, - dass also Graffiti eben nicht à corps perdu verständlich sind. ${ }^{15}$ Und gerade deshalb ist die Hegelsche Sprachtheorie wegweisend: Hegel zeigt, dass die Dimension der Sprache weder mit den Buchstaben beginnt, noch in ihnen kulminiert. Sprache entfaltet sich zu einer komplexen Sinndimension, die nicht auf eine Buchstabenmechanik reduziert werden kann. Sie wurzelt in einem Ausdrucksgeschehen des Leibes, d. h. in den keineswegs nur individuellen, sondern im intersubjektiven und zugleich vorsprachlichen Kommunikationszusammenhang habitualisierten „Gebärde[n]“116 und Gesichtsausdrücken. Die Dimension der Sprache, so zeigt Hegel, beginnt bereits weit vor der Buchstabenmechanik. Und sie wird deshalb von ihm konsequenterweise bereits im Rahmen der Anthropologie, d.h. noch vor der Explikation unserer Kulturtechniken behandelt - und zieht sich von dort aus durch die gesamte Geistdimension hindurch - bis hin zu dessen logischer Selbsterfassung.

15 Vgl. Beuthan: Was ist Graffiti? (wie Anm. 10).

16 Hegel: Werke. Bd. 10 (wie Anm. 12), S. 110. 
VI.

Fazit: Die Grenzen der Sprache sind für Hegel die Grenzen des Geistes. Damit ist zugleich gesagt: Die Grenzen sind nur Übergänge einer transmedialen Praxis. Und die Monstrosität der Buchstaben ist nur das Zeichen einer solchen Grenze - welche erst dann furchterregend wird, wenn man sie als unpassierbare Schranke, als äußerste Grenze missversteht (Fichte) oder zum opaken Ursprung fetischisiert (Kittler). - Was mit Hegels Einsicht am Ende gewonnen ist, mag man nun anhand der möglichen Urteile über Graffiti entscheiden:

(1) Während die fichtesche Position Graffiti als geistlos abtun dürfte, und (2) die romantische Position auf der Suche nach einer übersprudelnden Sinnfülle der Sprache ihren Mangel an Geist monieren dürfte, wird die (3) geistfeindliche Position ihre Geistlosigkeit begrüßen, ohne ihre Sinnhaftigkeit auch nur in Betracht zu ziehen. Gegen die geistfeindliche Position kann (4) der hegelsche Ansatz aber gerade die Sinnhaftigkeit der Buchstaben in den Blick bringen, welche sich aus der Leiblichkeit der Sprache speist. 

EVELINE CIOFLEC

\section{SCHRIFT ALS SINNSTIFTUNG}

\section{Eine soziokulturelle Fragestellung}

Schrift und Buchstaben stehen zwischen dem Vorwurf, die lebendige Rede zu verfestigen, und der preisenden Bestimmung als Vermittler und Traditionsstifter, als Geschichte ermöglichende Phänomene. Ein drittes Moment ist weniger vertreten, nämlich Buchstaben und Schrift als eigenständige Sinn- und Weltstifter zu betrachten. Die Strukturierung der Schrift und der Buchstaben, strukturiert gleichzeitig die Welt und den Sinn, den wir der Welt zuschreiben. Nicht allein das Symbolische, nicht allein die Vermittlung sind den als Schrift aneinandergereihten Buchstaben zuzuschreiben, sondern die Strukturierung von Sinngehalten. Von Platon schon als Nachteil gesehen, dass die Schrift den reinen Gedanken nicht wiedergeben kann, von Heidegger als Verfestigung gesehen, bieten die Schrift und deren Buchstaben und Zeichen die Möglichkeit einer neuen Sinndimension. Werden diese nicht allein als Wiedergabe von Gedanken und Rede betrachtet, so zeigt sich darin eine neuartige Dimension der Welt.

Das Lesen von Schriftzeichen impliziert meistens, dass man durch sie hindurch liest, die Buchstaben als solche gar nicht explizit wahrnimmt. Was tatsächlich vernommen wird, ist, der Sinn, den die Wörter machen, die Sprache, die sie fließen lassen, dasjenige worauf sie als Zeichen verweisen, hindeuten. Als solche verschwinden Buchstaben in der Schrift, und diese wird beim Lesen wiederum in Rede verwandelt. Was aus dieser Perspektive den Buchstaben ausmacht, ist, dass er als Zeichen über sich hinausweist, in die gesprochene Rede. Umgekehrt gilt, dass die gesprochene Rede mit Buchstaben wiedergegeben werden kann. Das Verhältnis ist allerdings nicht eins zu eins. Buchstaben bergen Sinngestaltung und damit meine ich nicht die graphisch-ästhetische Prägung der einzelnen Buchstaben, sondern ihre Zugehörigkeit zu einem System, das einen Sinnhorizont ausmacht. Um diesen Aspekt herauszuarbeiten, wende ich 
mich in einem ersten Schritt phänomenologischen Aspekten der Schrift und darin des Buchstabens zu.

Die Sinndimension der Schrift ist ferner in ihrer soziokulturellen Bedeutung zu besprechen. Im Vordergrund steht dabei nicht das Verhältnis von Buchstaben und Schrift zu den Gedanken, die die vermeintlichen Sinnträger sind, sondern vielmehr das Verhältnis zur Gesellschaft und Kultur, zur Welt als Sinngebilde. Jan Assmann unterscheidet treffend zwischen Schriftkultur und Schriftsystem, da allein ein Schriftsystem noch keine Schriftkultur ausmacht: Die Schriftkultur meint gerade die genannte Verwobenheit von Welt und Schrift und hat somit auch umfassendere Folgen wie Traditionsbildung, Vergangenheitsbezug und Identitätsbildung. ${ }^{1}$ So lassen sich anhand der Schrift Strukturfragen beantworten. Buchstaben werden hier allerdings nicht in ihrer Bildlichkeit, ${ }^{2}$ sondern als Teile der Schrift berücksichtigt, mit Bezug auf die Strukturierung von Sinn in der Schrift.

Seit jüngster Zeit ist ,Philosophie der Schrift' ein Zweig der Philosophie geworden, der vor allem, jenseits der Hervorhebung der Schriftbildlichkeit, im Rahmen des Mediendiskurses vermehrt Aufmerksamkeit erzielt hat und meist mit Blick auf den Aspekt der Medialität der Schrift, d.h. der Vermittlung vernommen wird. ${ }^{3}$ Die Medialität besteht im Wesentlichen darin, die Schrift, den Text als materielle Veräußerlichung der Rede aufzufassen, in der „die veräußerlichte sprachliche Handlung in der Schrift geronnen ist" ${ }^{\text {“ }}{ }^{4}$ Dieser Aspekt wird meistens

1 Vgl. Jan Assmann: Das kulturelle Gedächtnis: Schrift, Erinnerung und politische Identität in frühen Hochkulturen. München ${ }^{3} 2002$, S. 301. Assmann behandelt die Schriftkultur im Rahmen der Theorie des kulturellen Gedächtnisses, neben Traditionsbildung, Vergangenheitsbezug und Identitätsbildung.

2 ,Schriftbildlichkeit' ist in den vergangenen Jahren als eigenständiger Forschungsbereich immer mehr in den Vordergrund gerückt. Vgl. hierzu den Sammelband von Sybille Krämer, Eva Cancik-Kirschbaum, Rainer Totzke (Hrsg.): Schriftbildlichkeit: Wahrnehmbarkeit, Materialität und Operativität von Notationen. Oldenbourg 2012.

3 Vgl. Jürgen Villers: Über den Skriptomorphismus der Philosophie. In: Elisabeth Birk, Jan Georg Schneider (Hrsg.): Philosophie der Schrift. Tübingen 2009, S. 59-74.

4 Konrad Ehlich: Schrift, Schriftträger, Schriftform: Materialität und semiotische Struktur. In: Erika Graber, Konrad Ehlich, Jan-Dirk Müller (Hrsg.): Materialität und Medialität von Schrift. Bielefeld 2002, S. 91-111, hier S. 92. Wie im Weiteren bei Ehlich auch zu erkennen ist, durchbrechen 
auf den Phonozentrismus, d.h. auf die Sprache als gesprochene Rede, zurückgeführt, und setzt sich zuweilen auch in vergleichenden Analysen unterschiedlicher Sprachsysteme durch. ${ }^{5}$

Um der Frage nach dem Phonozentrismus nachzugehen, werde ich Martin Heideggers Auffassung hinzuziehen und, auf Jacques Derridas Kritik eingehen. Im Anschluss daran werde ich die Ausschließlichkeit der Schrift als Vermittlung hinterfragen und die Tragfähigkeit der Schrift als sinnbildende Struktur im soziokulturellen Zusammenhang thematisieren.

\section{PHÄNOMENOLOGISCHE BETRACHTUNG VON SCHRIFT IN BEZUG AUF REDE}

Phänomenologisch möchte ich da ansetzten, wo Buchstaben aufsässig werden und sich dem Zusammenhang gerade entziehen, ihrer Rolle als Medium für Gedanken zu funkieren entsagen und dann als solche, unleserlich' dastehen. Als Beispiel sei daran erinnert, wie wir gelegentlich bei einem Wort, sei es unbekannt oder einfach nur unschlüssig im Kontext, in Verlegenheit geraten und dessen Buchstaben sich einfach nicht aneinander reihen wollen - und wenn sie das endlich tun, merken wir, dass wir z. B. das ,e' in der Mitte des Wortes übersehen haben - so etwa bei gewissen Paronymen, wie ,Streich', das wir vielleicht zunächst als ,Strich' gelesen haben. ${ }^{6}$

Ein störrischer Buchstabe verweist auf einen Zusammenhang, dem er sich entzieht. Martin Heidegger spricht in Sein und Zeit von der Verbundenheit mit der Welt als Bedeutungszusammenhang. Dieser ist auf den

die Strukturen jeweiliger Schriftarten und semiotischer Implikationen der unterschiedlichen Schriftstrukturen diesen Rückbezug auf die gesprochene Sprache nicht, sondern setzen ihn eher voraus. Allerdings führt die Hervorhebung der Materialität der Schrift für eine semiologische Erfassung der Analogie der Phonologie als Graphematik auf eine genauere Beobachtung der einzelnen Schriften in ihrer struktureigenen Semantik: „Eine konsequente Entwicklung der Analogie erkennt just darin die Aufgabe einer systematischen transindividuellen Graphologie, die inneren semiotischen Entwicklungsstrukturen theoretisch zu erfassen, zu klassifizieren und in ihren jeweiligen Umsetzungen zu beobachten." (Ebd., S. 108)

5 Vgl. Zhenjiang Yan: Der geheime Phono- und Eurozentrismus des Redens von Schrift. In: Erika Graber, Konrad Ehlich, Jan-Dirk Müller: Materialität und Medialität von Schrift (wie Anm. 4), S. 151-164, hier S. 162. 6 Für eine Einteilung deutscher Paronyme vgl. Ioan Làzàrescu: Deutsche Paronyme. In: Grazer Linguistische Studien 43 (Frühjahr 1995), S. 81-95. 
„Um-zu“-Charakter von „Zeug“, d. h. von verwendbaren Dingen, zurückzuführen. Als Zuhandenes bedeutet ein Ding nicht in sich etwas, sondern erst mit Bezug auf ein anderes, für das es gebraucht oder verwendet wird? In diesem „Um-zu“-Charakter besteht die „Vertrautheit mit Welt", insofern als vertraut ist, wofür das eine oder andere Zuhandene eingesetzt wird. ${ }^{8}$

Die Vertrautheit mit der Welt lässt aber gerade nicht zu, dass Welt als solche sich in ihrer Vertrautheit bekundet. Erst wenn die Vertrautheit und der Bewandtnischarakter des Zeugs, der dem Dasein in der Struktur des „Um-zu“ zugänglich ist, durchbrochen sind, kündigt sich das Phänomen der Welt an. Weil dasjenige, was besorgt wird, aus dem Zusammenhang des Besorgens heraustritt (und dieses geschieht in der Auffälligkeit, Aufdringlichkeit und Aufsässigkeit des Zuhandenen ${ }^{9}$ ) tritt der Zusammenhang, als solcher hervor. Weil das Zuhandene nun unbrauchbar oder widerständig oder hervorstechend geworden ist, erscheint es als Vorhandenes. Allerdings erscheint es nur anders, weil es ehedem in einem Zusammenhang als Zuhandenes erschienen ist. Also fällt das Seiende so nicht nur als Vorhandenes auf, sondern es lässt zugleich den Zusammenhang, in den es gehört hat, erscheinen. Der Zeugzusammenhang, aus dem das Zuhandene als lediglich Vorhandenes hervortritt, macht noch nicht die Welt aus, kündigt aber so etwas wie Welt an: Nämlich die Welt als Zusammenhang. Dieser Zusammenhang wird hier von Heidegger von dem praktisch-hantierenden Umgang her gedacht, anhand eines Werkstatt-Modells: solange das Zuhandene der Tätigkeit dienlich ist, fällt es gar nicht auf und ergibt am Leitfaden der Tätigkeit den genannten, kohärenten Zusammenhang. ${ }^{10}$

Buchstaben können auch als verwendbares Zeug aufgefasst werden und dabei in ihrer werkzeugartigen Dinghaftigkeit durchleuchtet werden, ${ }^{11}$ was allerdings nicht bedeutet, sie darauf zu reduzieren. Aber

7 Martin Heidegger: Gesamtausgabe. Hrsg. von Friedrich-Wilhelm von Herrmann u. a. Frankfurt a. M. 1975 ff. (im Folgenden: GA), Bd. 2: Sein und Zeit (1927), S. 68 f., 78.

8 Ebd., S. 76.

9 Ebd., S. 74

10 Ebd., S. 70 f.

11 Dass Buchstaben und überhaupt Zeichen nicht strikt als „Werkzeug“ aufgefasst werden können, liegt auf der Hand, daher muss betont werden, dass es sich lediglich um eine Analogie handelt, anhand derer hier ein Aspekt der Buchstaben hervorgehoben wird. Hinzugezogen wird Heideggers Auffassung vom Seienden als Zeug in Anlehnung an das Werkzeug: „Ein 
insofern, als Buchstaben, verwendet' werden können, um eine Schrift zu konstituieren, und somit einer Zweckmäßigkeit dienen, können sie als werkzeugartig betrachtet werden. Der Verweisungscharakter der Buchstaben, die aufeinander bezogen sind, lässt zu, eine Analyse der Buchstaben mit jener des Werkzeugs bei Heidegger zu vergleichen. Was wir daraus gewinnen, ist der Gedanke eines bedeutsamen Zusammenhangs, in welchen die Buchstaben gehören und in dem sie funktional, dienlich sein können. Der Frage, in welchem bedeutsamen Zusammenhang sie gelesen werden, werde ich hier im Weiteren nachgehen.

Sicherlich sind Buchstaben dabei nicht einfach nur verwendbares Zeug im Sinne von Werkzeugen, sie sind Sinnträger - vereinzelt als Symbole, und zusammenhängend als Schrift. ${ }^{12}$ Werden sie allerdings allein in ihrem Ausdruckscharakter in Betracht gezogen, nämlich darin, dass sie Bedeutung verweisend wiedergeben, dann fungieren Buchstaben in ihrer ,Dienlichkeit'. Und gerade diesen Ausdruckscharakter unterstreicht Heidegger in einer späteren Stellungnahme, nämlich in Unterwegs zur Sprache: Sowohl die Schrift als auch die Rede werden hier als Ausdruck eines Sinns beschrieben. Sowohl die Schrift als auch die Rede werden als Verlautbarung einer bedeutsamen „Stille“ aufgefasst, d. h. aber als Verlautbarung von Sinn, um es hier in aller Kürze zu vermerken. ${ }^{13}$

Zeug ,ist‘ strenggenommen nie. Zum Sein von Zeug gehört je immer ein Zeugganzes, darin es dieses Zeug sein kann, das es ist. Zeug ist wesenhaft ,etwas, um zu ...'. Die verschiedenen Weisen des ,Um-zu' wie Dienlichkeit, Beiträglichkeit, Verwendbarkeit, Handlichkeit konstituieren eine Zeugganzheit. In der Struktur ,Um-zu' liegt eine Verweisung von etwas auf etwas.“ (Ebd., S. 68)

12 Einzeln können Buchstaben als Symbole stehen und sind dann auch als vereinzelte Sinnträger. Für Buchstaben als Symbole vgl. Klaus Schreiner: Buchstabensymbolik, Bibelorakel, Schriftmagie. Religiöse Bedeutung und lebensweltliche Funktion heiliger Schriften im Mittelalter und in der Frühen Neuzeit. In: Horst Wenzel, Wilfried Seipel, Gotthart Wunberg (Hrsg.): Die Verschriftlichung der Welt. Bild, Text und Zahl in der Kultur des Mittelalters und der Frühen Neuzeit. Wien und Mailand 2000, S. 59-103; ders.: Heilige Buchstaben, Texte und Bücher, die schützen, heilen, helfen. Formen und Funktionen mittelalterlicher Schriftmagie. In: Erika Graber, Konrad Ehlich, Jan-Dirk Müller (Hrsg.): Materialität und Medialität von Schrift. Bielefeld 2002, S. 73-89.

13 Heidegger: GA 12: Unterwegs zur Sprache, S. 31. 
Noch aussagekräftiger für Heideggers Auffassung vom Verhältnis zwischen Schrift und Sinn, ist seine Hervorhebung mit Bezug auf Aristoteles, in seiner ersten Marburger Vorlesung, Einführung in die Phänomenologische Forschung:

Es gibt nun Laute, die etwas kundgeben, ohne etwas zu bedeuten, ảypá $\mu \mu \alpha \tau o$ (Aristoteles, De Interpretatione, 2, 16 a 28 sq.) z. B. das Stöhnen. Es fehlt diesen die Prägung, so dass man sie schreiben oder lesen könnte; es geht nur auf Grund der Bedeutung. ${ }^{14}$

Aus dieser Interpretation des aristotelischen $\lambda$ ó $о \varsigma$ geht die Bedeutung als vorrangig in Bezug auf die Schriftzeichen, auf die Zusammengehörigkeit der Silben hervor. „Das Zusammensein von bestimmten Silben ist erst durch die einheitliche Bedeutung gestiftet. ${ }^{“ 15}$ Der Vorrang der Bedeutung, der letztlich auf den Vorrang des $\lambda$ ó bei Aristoteles für die gesprochenen Sprache festgehalten wird, bestimmt auch die Auffassung von Schriftzeichen, die auf Grund der Bedeutung, die sie verkörpern, zu lesen sind, zumal diejenigen Laute, denen keine Bedeutung zukommt, auch nicht geschrieben werden können. Die phonetische Schrift behält somit, als Notation, die Nähe zur bedeutsamen, gesprochenen Rede bei und darin ist sie tückisch, d.h. sie verweist immer auf ein anderes. Die Funktion der Buchstaben Zeichen, für einen Laut zu sein, führt zurück auf die Stimme: Hinter den Phänomenen steht die Stimme, die Verlautbarung.

Weil sich in der niedergeschriebenen Philosophie der Grad der ,Beweglichkeit' des Verstehens einschränken muss, bleibt es, so Heidegger, stets fraglich, wie viel von den eigentlichen Intentionen durch die Schrift (und mehr noch die publizierte Schrift) mitgeteilt werden kann: ${ }^{16}$ „Im

14 Heidegger: GA 17: Einführung in die Phänomenologische Forschung, S. 17. 15 Ebd.

16 Darin finden wir ein platonisches Moment der Schriftkritik wieder, wie Platon diese im siebten Brief formuliert: Platon: Siebter Brief. In: Rainer Knab (Hrsg.): Platons Siebter Brief: Einleitung, Text, Übersetzung, Kommentar. Hildesheim, Zürich und New York 2006, 341a-344c, insbes. 344. Für Platon ,ist jeder ernsthafte Mann weit davon entfernt, über die bedeutenden Dinge jemals etwas zu schreiben und so unter den Menschen der Mißgunst und Ratlosigkeit auszusetzen." (344 b-c) Begründet wird dieses damit, dass das, was die fünfte Stufe der Erkenntnis ausmacht, nur nach großer Mühe und Übung in den vier Stufen der Erkenntnis erlangt 
Schriftlichen büßt das Denken leicht seine Beweglichkeit ein. Vor allem aber kann es da nur schwer die ihm eigene Mehrdimensionalität seines Bereiches innehalten. ${ }^{\text {"17 }}$ Gleichzeitig ist aber auch das Sagen, welches das jeweilig in einem Text Gemeinte benennt, oder, wie er es später nennt, „die Sage“, nicht „Ausdruck des Denkens“, sondern „selber sein Sang und Gang“..18

Für Heidegger ist nicht die Aussage der Wahrheitsträger, sondern die Wahrheit benennt das Hervorbringen aus der Verborgenheit. ${ }^{19}$ Dieses

werden kann: „Denn wenn einer davon nicht einigermaßen zumindest die vier Stufen erfaßt hat, wird er niemals vollkommen des Wissens um das fünfte teilhaftig sein.“ (342 d-e). Von den fünf Stufen heißt es: „Es eignet jedem der seienden Dinge dreierlei, wodurch sich die Erkenntnis einstellen muß, das vierte aber ist sie selbst - als fünftes jedoch muß man eben das setzen, was genau das Erkennbare und wirklich Seiende ist; das eine ist die Bezeichnung, das zweite die Definition, das dritte das Abbild, das vierte aber die Erkenntnis.“ (342 a-b) Außerdem kann das Gedachte nicht „auf etwas Unveränderlichem, was genau ja dem in Buchstaben geschriebenen widerfährt“ (343 a) gegründet werden kann, d. h. aber auch nicht schriftlich vermittelbar ist (vgl. Knabs Kommentare zu 342d1-2 und 342d2, ebd., S. 272-3). „Es eignet jedem der seienden Dinge dreierlei, wodurch sich die Erkenntnis einstellen muß, das vierte aber ist sie selbst - als fünftes jedoch muß man eben das setzen, was genau das Erkennbare und wirklich Seiende ist; das eine ist die Bezeichnung, das zweite die Definition, das dritte das Abbild, das vierte aber die Erkenntnis.“ (342 a-b, vgl. 342 d-f.)

17 Heidegger: GA 9: Holzwege, S. 315; und ferner: „Die Strenge des Denkens besteht im Unterschied zu den Wissenschaften nicht bloß in der künstlichen, das heißt technisch-theoretischen Exaktheit der Begriffe. Sie beruht darin, daß das Sagen rein im Element des Seins bleibt und das Einfache seiner mannigfaltigen Dimensionen walten läßt. Aber das Schriftliche bietet andererseits den heilsamen Zwang zur bedachtsamen sprachlichen Fassung.“ (ebd.) Der „heilsame Zwang“ ist doppeldeutig und vermag es kaum, Heideggers sonst recht ablehnende Haltung der Schrift gegenüber zu entkräften.

18 Heidegger vermerkt, dass das Denkwürdige als die „Sage des Denkens [...] ist nicht der Ausdruck des Denkens, sondern es selber, sein Gang und Sang.“ (Ebd., S. 424) Heidegger umschreibt seinen eigenen Versuch in seinem Beitrag ,Zur Seinsfrage' als ein Versuch, dasjenige, was im auszulegenden Text „im schreibenden Sagen zu erweisen verstattet“ ist, „in eine höhere Mehrdeutigkeit zu heben“ (ebd., S. 418). Texte, zumindest gewisse Texte, liest er somit als ,schreibendes Sagen'. Das Sagen meint hier allerdings nicht mehr auf die gesprochene Rede, sondern eher die Bedeutung des Geschriebenen, also dasjenige, was in einem Text mitgeteilt, vermittelt wird.

19 Heidegger: GA 2: Sein und Zeit, S. 219. 
geschieht zwar auch sprachlich, aber nur solange die Sprache nicht festhalten bedeutet, sondern gerade, so könnte man sagen, so nah wie möglich an der gesprochenen Rede ist. Das Schweigen wird von hier aus als Möglichkeit der Rede aufgefasst. ${ }^{20}$ Somit wird das Schweigen bei Heidegger nicht negativ gewertet, wie etwa bei Platon das Schweigen der Texte verstanden werden könnte, worauf ich weiter unten noch zurückkomme, sondern es meint positiv „etwas zu sagen haben“. ${ }^{21}$ MerleauPonty drückt diesen Aspekt der Rede, dass sie immer einen Überrest beibehält, der nicht selbst auch noch gesagt oder geschrieben werden kann, noch pointierter aus:

Wenn wir nun aber die Vorstellung von einem ursprünglichen Text aufgeben, dessen Übersetzung oder chiffrierte Version unsere Sprache wäre, werden wir erkennen, dass die Vorstellung von einem vollständigen Ausdruck unsinnig ist, dass jede Rede indirekt oder anspielend, und wenn man so will, Schweigen ist. ${ }^{22}$

Das wesentliche Merkmal der Rede ist, dass sie im Vollzug stattfindet, und dieser Vollzug geht bei Merleau-Ponty auch in das Schreiben über:

Unsere Analysen des Denkens tun so, als ob dieses, bevor es seine Wörter gefunden hat, schon eine Art idealer Text wäre, den unsere Sätze zu übersetzen suchten. Aber der Autor hat keinen Text, den er mit seiner Schrift konfrontieren könnte, keine Sprache vor der Sprache. ${ }^{23}$

Die Bedingungen der Ausdrucksweise, so Merleau-Ponty, definieren sich im Vollzug und nicht anhand eines Modells. Gerade weil Gesprochenes nicht festgehalten werden kann, da lebendige Rede immer schon mehr beinhaltet als das Gesagte oder das Geschriebene, was sowohl bei Heidegger, als auch bei Merleau-Ponty zu lesen ist, werden das Schweigen oder die Stille, und mehr noch, das Intervall zwischen den Wörtern, wie MerleauPonty dieses vermerkt, für die Sinnerschließung ausschlaggebend:

20 Ebd., S. 164, 296.

21 Ebd., S. 165.

22 Maurice Merleau-Ponty: Das mittelbare Sprechen und die Stimme des Schweigens. In: ders.: Das Auge und der Geist. Hamburg 1984, S. 69-114, hier, S. 73 .

23 Ebd., S. 72. 
Was die Sprache angeht, wenn sie die laterale Beziehung von Zeichen zu Zeichen ist, die jedes von ihnen bedeutend macht, so taucht der Sinn erst im Schnittpunkt und gleichsam im Intervall der Wörter auf. ${ }^{24}$

In der späteren Philosophie Heideggers tritt in der Auslegung der Sprache, zu dem Moment der Rede eine neue Wendung hinzu. Denn, wenn beim frühen Heidegger die im Schweigen begründete Rede, d. h. die Rede in ihrem eigentlichen Modus das ist, worin Philosophie sich artikuliert, so ist im späteren die Stille dasjenige, das die Sprache und somit auch die Philosophie trägt. Aber auch hier geht es um die Sprache, die spricht, um die Lebendigkeit des Gesprochenen, wie auch schon im frühen Heidegger das „,Mitgehen“ mit dem Leben“25, der Vollzug des Philosophierens zentral war. ${ }^{26}$ Dass für Heidegger die Schrift als solche nicht zentral war, sondern eher die Rede oder auch die Verlautbarung oder eben die Stille aus der die Dichtung erwächst, ist kaum anzuzweifeln. ${ }^{27}$ Weder in den frühen, noch in den späteren Auffassungen, ist die Schriftlichkeit für Heidegger ausschlaggebend.

Jaques Derrida hat später in seinem Text La voix et le phénomène diesen Aspekt als Phonozentrismus der Phänomenologie und der philosophischen Tradition hervorgehoben ${ }^{28}$ und in Frage gestellt, ob die Stimme als Verlautbarung von Sinn allein Träger von Bedeutungen sein kann. In diesem Text unternimmt Derrida eine Analyse im Anschluss

24 Ebd., S. 71.

25 „Mitgehenkönnen - Vertrautsein - ,Liebe‘. Liebe als Motivgrund des phänomenologischen Verstehens - in seinem Vollzugssinn notwendig mitgegeben." (Heidegger: GA 58: Grundprobleme der Phänomenologie [1919/20], S. 185)

26 Vgl. Heidgger: GA 61: Phänomneologische Interpretationen zu Aristoteles. Einführung in die Phänomenologische Forschung, S. 41-61.

27 Für die Dichtung ist die Schriftlichkeit nicht ausschlaggebend, sondern vielmehr der Bezug zum Sagbaren. Es kommt nicht darauf an, ob sie geschrieben oder vorgetragen ist, sondern es kommt vielmehr auf die Bedeutung des Gedichteten an. Dem literarischen, für die Öffentlichkeit bestimmten Aspekt des Niedergeschriebenen steht Heidegger allerdings kritisch gegenüber. Vgl. hierzu: Diana Aurenque: Literatur, Öffentlichkeit und Geheimnis. Die Heideggersche Unterscheidung von geschrieben-ausgesprochenem und schweigend-hörendem Wort. In: David Espinet (Hrsg.): Schreiben Dichten Denken. Zu Heideggers Sprachbegriff. Frankfurt a. M. 2011, S. 13-28, hier S. 14-17.

28 Vgl. auch Günter Figal: Gegenständlichkeit. Tübingen 2006, S. 270. 
an Husserls Logische Untersuchungen und verweist auf die vorausgesetzte Unmittelbarkeit der Stimme als phoné..$^{29}$ Allerdings sieht er in der Stimme allein keine Differenzierung, keine Bedeutung, das heißt sie kann auch kein Verstehen tragen. ${ }^{30}$ Der Träger des Sinnes wird damit die Schrift. ${ }^{31}$

Vorgeworfen wird Derrida, dass er der Schriftverherrlichung in der abendländischen Geschichte, etwa in der Wissenschaft, in der westlichen Tradition nicht gerecht wurde..$^{32}$ Der Logozentrismus als Phonozentrismus im Sinne der Vorrangigkeit der Rede, hat das abendländische Denken geprägt und dennoch ist die Schrift kaum außer Acht gelassen worden, man denke an religiöse Schriften oder an die Rolle der Schrift in der Gesetzgebung etc. Die Unmittelbarkeit der Rede lässt diese allerdings als vorrangig erscheinen.

29 Vgl. Jacques Derrida: Die Stimme und das Phänomen. Ein Essay über das Problem des Zeichens in der Philosophie Husserls (1967). Frankfurt a. M. 1979, S. 133-137.

30 Günter Figal wendet in diesem Punkt ein, dass die Stimme wesentlich Mitteilung ist, demnach also durchaus eine Differenz in sich trägt, was hier nicht weiter verfolgt werden kann, aber dennoch angeführt werden soll: „Nach dieser Beschreibung ist die Stimme ihrem Wesen nach für sich. Aber ihr Verlauten will zur schweigsamen Einsamkeit des Seelenlebens, mit der Derrida sie assoziiert, nicht recht passen. Vielmehr ist die Stimme ihrem Wesen nach auf Mitteilung eingestellt [...] Das Sprechen mit anderen ist für die Stimme keine Unterbrechung ihrer Selbstaffektion, sondern Normalität. [...] Das heißt nicht, daß Derridas Konzeption gänzlich verfehlt sei. Die Erfahrung der Stimme, wie er sie beschreibt, gibt es; mit der eigenen Stimme erfährt man immer auch sich. Doch wie es sich mit dieser Selbsterfahrung verhält, läßt sich nur angemessen beschreiben, wenn man auf das Mitteilungswesen der Stimme eingeht.“ (Figal: Gegenständlichkeit, wie Anm. 28, S. 277)

31 Derrida: Die Stimme und das Phänomen (wie Anm. 29), S. 142.

32 Vgl. Georg P. Khushf: Die Rolle des ,Buchstabens' in der Geschichte des Abendlands. In: Hans Ulrich Gumbrecht und K. Ludwig Pfeiffer (Hrsg.): Schrift. München 1993, S. 21-34: Entgegen den von Derrida hervorgehobenen Logozentrismus der westlichen Kultur, hebt Khushf hervor dass: „Die westliche Vorstellung einer vom Sprechen vereinnahmten Schrift geht in Wirklichkeit auf das Modell der Schrift zurück.“ (S. 25) „Die Untersuchungen von Ong und Havelock, unter anderen, haben, wie es scheint, Derrida und den ganzen traditionellen ,klassischen' Begriff des Sprechens grundsätzlich in Frage gestellt.“ (S. 25) Die Vormachtstellung des Sprechens, so Khushf, könnte, so wie sie von Derrida hervorgehoben wird, möglicherweise von der Schriftlichkeit bereits geprägt sein. (S. 25) 
Paradigmatisch für die Schriftkritik ist Platos Stellungnahme. Bekanntermaßen weist Plato der Schrift die Funktion der Gedächtnisstütze, der Erinnerung an dasjenige, was man bereits weiß, als positive Eigenschaft zu..$^{33}$ Auf der anderen Seite hat die Schrift laut Platon eine negative Wirkung auf die Fähigkeit etwas beizubehalten: Schriften führen zu Trägheit des Gedächtnisses, da das Wissen nicht mehr lediglich in der Seele ist, sondern nachgelesen werden kann und somit keine Not mehr ist, dieses in der Seele beizubehalten. ${ }^{34}$ Der größte Einwand Platos ist allerdings jener, dass die Texte schweigen. ${ }^{35}$ D. h. sie können sich nicht verteidigen und sie können nicht antworten, wenn man sie befragt. ${ }^{36}$

Gadamer hat dagegen den Einwand erhoben, dass das Textverständnis, wie jedes hermeneutische Phänomen, „nach dem Modell des Gespräches, das zwischen zwei Personen statthat" betrachtet werden kann, wobei die leitende Gemeinsamkeit eines Gesprächs zwischen zwei Personen und dem Textverständnis ist, dass ,jedes Verstehen und jede Verständigung im Gespräch eine Sache im Auge hat.$^{37}$ Aber auch diese Position bleibt nicht unumstritten: schlussendlich können Texte tatsächlich nicht antworten, wie ein Gegenüber antworten würde. ${ }^{38}$

33 Plato: Sämtliche Werke in zehn Bänden. Griechisch und Deutsch. Frankfurt a. M., Leipzig 1991, Bd. VI: Phaidros, 275d.

34 Ebd., 275 a.

35 Ebd., 275 d.

36 Günter Figal verweist auf eine andere mögliche Interpretation der Schrift-Auffassung bei Platon: Es handelt sich bei Platon nicht um eine radikale Kritik an der Schrift, sondern vielmehr um eine Andersartigkeit der Schrift: „Die Kritik an der Schrift, als welche diese Gedanken meist verstanden werden, sind sie freilich nur unter einer Voraussetzung: daß die Schrift an der Rede gemessen wird.“ (Figal: Gegenständlichkeit, wie Anm. 28, S. 72) Und vom Schweigen der Texte heißt es ferner: „Schriften gehören nicht in den Zusammenhang menschlichen Lebens; sie sind keine Personen. Wer von ihnen erläuternde Auskunft möchte, fällt auf den Schriftbestand zurück.“ (Ebd.) Texte geben allerdings dem Denken „etwas, das es bei sich selbst und durch sich selbst nicht findet: einen Sinn der nicht seine eigene Richtung ist.“ (Ebd., S. 73)

37 Hans Georg Gadamer: Wahrheit und Methode. Grundzüge einer philosophischen Hermeneutik. Tübingen ${ }^{6}$ 1990, S. 384: „Wie einer sich mit seinem Gesprächspartner über eine Sache verständigt, so versteht auch der Interpret die ihm vom Text gesagte Sache."

38 Vgl. hierzu Figal: Gegenständlichkeit (wie Anm. 28), S. 72 f. 
Auch kann die Wahrheit laut Platon nicht in Texten beibehalten werden, sondern nur in der Seele. Wer seine wahren Ideen zu Papier bringt, und somit der Masse zugänglich macht, der hat nicht verstanden, dass er sie damit des Privilegs beraubt und dass sie als Wahrheit verkehrt werden, da die Leser mindestens wegen mangelnder Übung nicht auf gleicher Höhe sind, um zu verstehen, was der Autor niedergeschrieben hat. Daher ist es ratsam, die letzten Wahrheiten gar nicht zu Papier zu bringen. ${ }^{39}$

Aus diesen Gründen lehnt Platon die Schrift eher ab, auch wenn er ihr die Nützlichkeit im Staat grundsätzlich zuerkennt. Die Oralität der Philosophie wird aber vorgezogen, da das Ziel der Bezug zu den Ideen ist. Dieser Grundgedanke wird prinzipiell beibehalten und wird auch noch im 17. und 18. Jahrhundert vertreten, etwa von Descartes, wenn das Bewusstsein als "einsame Rede“ im „einsamen Seelenleben“ betrachtet wird, wie später in den Logischen Untersuchungen Husserls.$^{40}$ Es ist demnach nicht schwer, wie Derrida, einen gewissen Logozentrismus als Phonozentrismus zu vermerken ${ }^{41}$ der die Geistigkeit der Philosophie, sei es mit Bezug auf die Ideen oder auch mit Bezug auf das Bewusstsein, hervorhebt.

39 Vgl. Platon: Siebter Brief (wie Anm. 16), 344 a-c; Fragen und Antworten und somit aufwendige Übung erst zu Einsicht und gedanklichem Erfassen. „Gerade deshalb ist jeder ernsthafte Mann weit davon entfernt, über die bedeutenden Dinge jemals etwas zu schreiben und so unter den Menschen der Mißgunst und Ratlosigkeit auszusetzen." (344 b-c)

40 Die Bedeutung (Sinn) des Ausdrucks fällt nicht mit seiner kundgebenden Funktion zusammen, was Husserl anhand der Rede im einsamen Seelenleben aufweist. Vgl. Edmund Husserl: Husserliana Bd. XIX: Logische Untersuchungen. Zweiter Teil. Untersuchungen zur Phänomenologie und Theorie der Erkenntnis. In zwei Bänden. Hrsg. von Ursula Panzer. Halle ${ }^{3} 1984$ (1901, 1922), S. 42: Das Reden mit sich selbst, der innere Monolog gibt nichts kund und dennoch ist im einsamen Seelenleben, „die einsame Rede“, als vorgestellte ohne wirkliche Worte bedeutungsvoll. Im einsamen Seelenleben, in der inneren Rede, sind es gar nicht Worte, welche diese innere Rede darstellen, sondern Vorstellungen, welche nicht auf Worte angewiesen sind. Man stellt sich nicht das Wort, sondern unmittelbar den Gegenstand vor. „Nicht der Phantasierte Wortklang oder die phantasierte Druckschrift existiert, sondern die Phantasievorstellung von dergleichen." (Ebd.).

41 Vgl. Jacques Derrida: Grammatologie. Übersetzt von Hans-Jörg Rheinberger und Hanns Zischler. Frankfurt a. M. ${ }^{4} 1992$ (1967), S. 25; eingeführt wird diese Differenz wie folgt: „Die Differenz (différance) schlechthin wäre zwar ,ursprünglicher', doch könnte man sie nicht mehr ,Ursprung' und auch nicht ,Grund' nennen. Denn diese Begriffe gehören wesensmäßig in die Geschichte der Onto-Theologie, das heißt in das System, das als Auslöschung der Differenz fungiert." (ebd.) 
Den Phonozentrismus kritisiert Derrida, wenn er in der Grammatologie die Schriftlichkeit in Bezug auf die Mündlichkeit der Sprache zur Diskussion bringt, und beide auf eine „,ursprünglichere“ Differenz“ zurückführt, die als „Differieren“ aufgefasst wird. ${ }^{42}$ Damit greift er eine von Hegel schon angesprochene Differenz auf, die dieser allerdings als das „Sinnliche der Rede auf die Form der Allgemeinheit gebracht“, ${ }^{43}$ auf die Idealität der Sprache, bzw. auf den Namen als „einfachen Gedanken“ zurückverweist. ${ }^{44}$ Für Hegel ist die Dynamik des in der Schrift wiedergegebenen Sinns auf die Dynamik des Denkens zurückzuführen, weshalb auch nur eine nicht verbildlichende Schrift, sondern nur eine Zeichen-Schrift dieser Dynamik entsprechen kann ${ }^{45}$ Derrida knüpft allerdings nicht an die Idealität der Sprache, sondern hebt die différance hervor, die Differenz, die vor aller ontologischen Differenz von Sein und Seiendem liegt. ${ }^{46}$ Der Hintergrundgedanke der angeführten Kritik Derridas ist, dass die Oralität auf einer Präsenz der Rede beruht, auf einer Hervorhebung der Präsenz als Zeitigung, die als solche die abendländische Metaphysik durchherrscht, sich in der Form der Stimme durchgesetzt hat und in der Form des Bewusstseins als Selbstbewusstsein in der Moderne ausgeprägt hervortritt. ${ }^{47}$

Derrida geht es demnach vor allem um den ontologischen Wert der Schrift, der bei den Philosophen unterschätzt wurde. Die Differenz, die nicht reduzierbar ist, keiner Synthese unterzogen werden kann, als

42 Ebd., S. 44.

43 Georg Wilhelm Friedrich Hegel: Gesammelte Werke, Bd. 20: Enzyklopädie der philosophischen Wissenschafgten im Grundrisse (1830). Hrsg. von Wolfgang Bonsiepen und Hans-Christian Lucas. Hamburg 1992, S. 456, 28.

44 Ebd., S. 457, 21. Der Name ist für Hegel das Gemeinsame von Tonsprache und Buchstabenschrift, wobei „der Name ist das einfache Zeichen für die eigentliche, d. i. einfache, nicht in ihre Bestimmung aufgelößte und aus ihnen zusammengesetzte Vorstellung." (S. 457, 32-34) Darin unterscheidet sich für Hegel die Buchstabenschrift etwa von der Hieroglyphenschrift.

45 Vgl. Derrida: Grammatologie (wie Anm. 41), S. 45-48. Für Derrida ist Hegel nicht nur „der letzte Philosoph des Buches und der erste Denker der Schrift“, sondern „auch der Denker der irreduziblen Differenz“ (ebd., S. 48). Vgl. Hegel: Enzyklopädie (wie Anm. 43), S. 453-458 (\$459);

46 Vgl. Derrida: Grammatologie (wie Anm. 41), S. 44: „Seiend und Sein, ontisch und ontologisch, ,ontisch-ontologisch' wären im Hinblick auf die Differenz in originaler Weise abgeleitet auch in bezug auf das, was wir später die Differenz (différance) nennen werden."

47 Vgl. Derrida: Die Stimme und das Phänomen (wie Anm. 29), S. 137. 
geschichtliche Ur-differenz eine Differenz ist, die kein Zentrum, auf welches sie sich beziehen könnte, hat, prägt Derridas Auffassung. Für Derrida ist die différance eine Antwort auf den Logozentrismus bzw. dessen Alternative. So hält Hawthorn fest, dass der Logozentrismus auf Bedeutungen aufbaut, die auf eine Präsenz oder einen Ursprung zurückzuführen sind. Die différance aber ist „eine permanente Aufschiebung der Bedeutung, insofern als die Bedeutung durch den Unterschied anderen Bedeutungen gegenüber bestimmt und durch diesen Unterschied hervorgebracht wird, also flüchtig und nicht stabil ist. Bedeutung ist immer relational und niemals selbstpräsent bzw. selbstkonstituierend“ “48

Die Spur als différance, ${ }^{49}$ die "die Artikulation des gesprochenen Wortes und der Schrift" ermöglicht, ${ }^{50}$ ist für Derrida auch schon in der vermeintlich selbstpräsenten Stimme als Bewusstsein zu finden, ${ }^{51}$ da die hier vorausgesetzte unmittelbare Selbstpräsenz ebenfalls in einer Differenz begründet ist..$^{52}$ Der Phonozentrismus ist mit Derrida somit auf eine fälschliche Annahme einer Selbstpräsenz in der Erfahrung der Stimme begründet, deren Unmittelbarkeit durchaus hinterfragt werden kann.

\section{SOZIOKULTURELLE BEDEUTUNG DER SCHRIFT. EIN BEISPIEL.}

Einen Schritt dahin, die Schrift nicht in Gegenüberstellung zur gesprochenen Rede aufzufassen, d.h. sie nicht allein aus dem vollzogenen Selbstbezug, sondern als Teil der Welt zu betrachten, wäre, sich dem soziokulturellen Zusammenhang als bedeutsamen Zusammenhang, in dem die Schrift auftritt, zuzuwenden. Zu betrachten ist, wie die Schrift und deren Buchstaben tatsächlich in der Welt anzutreffen sind und wie sie die Welt bestimmen oder umstrukturieren. Ausgehend von Derridas Hinterfragung der auf die Verlautbarung zurückgeführten Schrift, soll hier nun die Schrift in ihrer Materialität hervorgehoben werden und somit ein

48 Jeremy Hawthorn: Grundbegriffe moderner Literaturtheorie. Tübingen, Basel 1994, S. 59.

49 Vgl. Derrida: Grammatologie (wie Anm. 41), S. 109: „Die (reine) Spur ist die Differenz (différance). Sie ist von keiner sinnlich wahrnehmbaren, hörbaren oder sichtbaren, lautlichen oder graphischen Fülle abhängig, sondern ist im Gegenteil deren Bedingung.“

50 Ebd., S. 110.

51 Ebd., S. 137 u. S. 142.

52 Ebd., S. 140. 
erster Schritt in die Richtung einer Ausarbeitung der Schrift-spezifischen Strukturierung und Sinnstiftung getan werden, wobei hiermit die Schrift „im geläufigen Sinn“ gemeint ist..$^{53}$

Die Schrift wird, wie wir sehen werden, in diesem Kontext als Machtinstrument gedeutet. Handelt es sich bei diesem Aspekt tatsächlich erst um die Schrift oder doch eher um die Sprache? Die Ur-Schrift ist, so gibt Derrida zu bedenken, der Sprache vorgängig, so dass „Sprache nie unversehrt, nie unberührt von der Schrift war; daß sie selbst schon immer eine Schrift gewesen ist. ${ }^{\text {"54 }}$ Bei diesem Gedankenexperiment zur Schrift als Machtinstrument, handelt es sich um eine konkrete, wenn auch an sich nicht fassbare und im strengen Sinne gerade nicht bestimmbare Spur. ${ }^{55}$ Das gilt im erweiterten Sinne auch für die Buchstaben: sie sind zwar konkret und im weiten Sinne sogar ,materiell' und können in ihrer Textur auch studiert werden, wobei Studien zur Aussagekraft der Ästhetik der Buchstaben gemacht werden können, sowie religiöse oder politische Wirksamkeit gewisser Buchstaben-Symbole erforscht werden können. Dabei sind allerdings die Systeme der Schrift und Bedeutung, in die sie eingebunden sind, niemals abgeschlossen, sondern differenzieren sich selbst stets aus.

So können Buchstaben jederzeit, als Konventionen, ersetzt werden, oder ausgetauscht werden. Platon hat bekanntlich im Dialog Kratylos die Konventionalität von Namen sowie jene der Buchstaben betont ${ }^{56}$ und die Konventionalität noch deutlicher im bereits erwähnten "Siebten Brief“ hervorgehoben. ${ }^{57}$ Um die Konventionalität der Buchstaben und der Schrift zu veranschaulichen, lohnt sich allerdings ein Blick in die Graphematik. Die Schreibweise des Deutschen war noch im 18. Jahrhundert, wie dieses z. B. an Inschriften zu erkennen ist, nicht normiert. Zu jener Zeit war

53 Ebd., S. 110.

54 Ebd., S. 99.

55 Diese ist nicht fassbar, da sie nicht auf eine Präsenz reduziert werden kann und somit nicht objektiviert werden kann. Vgl. ebd. Als ,irreduzible Ursynthese' ist sie sowohl in der Form des ,graphischen' als auch des ,nicht-graphischen Ausdrucks‘ am Werk. Vgl. ebd., S. 105.

56 Vgl. Plato: Sämtliche Werke (wie Anm. 33), Bd. III: Kratylos, 434 a.

57 Vgl. Platon: Siebter Brief (wie Anm. 16), 343 a-b: „Für nichts davon ist - so sagen wir - irgendeine Bezeichnung sicher, hindert doch nichts daran, dass das, was jetzt rund genannt wird, gerade heißt und das Gerade folglich rund, und es wird ebenso beständig andauern für diejenigen, die eine Bezeichnung verändert haben und es auf entgegengesetzte Weise benennen.“ 
die Schreibung - anders als heute - noch nicht normiert. Mit anderen Worten: für die Schreiber dieser Zeit blieb der Text sehr nah an der gesprochenen Sprache. Ein Schreiber konnte sogar beim Wiederholen ein und desselben Wortes in einem Text mehrere Schreibvarianten dafür wählen, solange der Leser in den entsprechenden, mitunter stark variierenden Buchstabenfolgen das beabsichtigte Wort erkannte..$^{58}$

Der Abstand zwischen den Buchstaben, aber auch unzählige andere Normen, wurden im Laufe der Zeit eingeführt. ${ }^{59}$ Würde man Buchstaben einfach übereinander schreiben, etwa wie in Palimpsesten, könnte man gar nicht mehr erkennen, was geschrieben steht.60

Die Konventionalität der Buchstaben und der Schrift ,im geläufigen Sinn “61 führt uns zurück auf die Frage nach der Tragfähigkeit der Schrift. Wird die Frage danach gestellt, wie Rousseau die Frage nach dem Ursprung der Sprache gestellt hat, ${ }^{62}$ so erweist sich die Schrift im ersten Schritt durch ihre Konventionalität als Entfremdung der lebendigen Rede, des Unmittelbaren der Rede. Wie die Schrift eine eigene Sinndimension als Aspekt der Welt stiftet, geht daraus allerdings nicht hervor und das Eigene der Schrift ist damit noch nicht thematisiert. So meint Frage nach der Tragfähigkeit eher, welche Macht die Schrift in der Gesellschaft ausübt und inwiefern sie das Leben der Menschen beeinflusst, mehr noch, welchen ontologischen Status die Schrift hat. Auch sind die Überlegungen zur Tragfähigkeit der Schrift nicht schriftinhärente Überlegungen, sondern Überlegungen über die Stellung der Schrift im Zusammenhang der Kultur und im Zusammenhang der Gesellschaft, Geschichte etc. In

58 Sorin Gadeanu: Graphematik und ,Phonetologie‘. Eine Schrift- und Lautlehre des Deutschen. Bucuresti 2009, S. 18.

59 Für einen zusammenfassenden Überblick zur Geschichte der deutschen Rechtschreibung vgl. Hermann Schreuninger: Geschichte der deutschen Rechtschreibung. Ein Überblick. Wien 1996.

60 Vgl. Christian Stetter: Die Groß- und Kleinschreibung im Deutschen. Zur sprachanalytischen Begründung einer Theorie der Orthographie. In: ders. (Hrsg.): Zu einer Theorie der Orthographie. Tübingen 1990, S. 200. 61 Derrida: Grammatologie (wie Anm. 41), S. 196-220.

62 Jean-Jacques Rousseau: Essai sur l'origine des langues. Hrsg. von Angèle Kremer-mariette. Pris 1974 (1781), S. 85-186. Rousseau vermerkt, dass die Sprache primär der Kommunikation gedient hat und dient, und somit bereits eine soziale Wirklichkeit ist und nicht primär das Denken ausmacht: „la parole, étant la première institution sociale, ne doit sa forme qu'a des causes naturelles.“ (S. 85; vgl. auch ebd., 1. Endnote) 
diesem Kontext, der wiederum in den weiter oben angesprochenen Bereich der Philosophie der Schrift gehört, erweist sich die Schrift, wie auch die Sprachlichkeit überhaupt, als genauso prägendes Moment der Kultur.

Lévi-Strauss erzählt und bespricht in den Traurigen Tropen die ,Schreibstunde', eine Szene aus seiner Erfahrung mit dem Stamm Nambikwara in den Amazonas: ${ }^{63}$ Der Anthropologe berichtet, dass die Nambikwaras mit Sicherheit weder schreiben, noch zeichnen konnten, mit Ausnahme einiger Zick-Zack Linien auf ihren Kürbissen. ${ }^{64}$ Bei einem Austausch von Geschenken, bei dem er ihnen Bleistifte und Papierblätter ausgeteilt hatte, stellte er eines Tages fest, dass sie den Bleistift als Schreibinstrument zu nutzen versuchten, auch wenn sie nur Striche damit zogen. Davor hatte der Anthropologe bei diesen keine Schrift beobachtet. ${ }^{65}$ Das Schreiben, so schlussfolgert Lévi-Strauss, hatte bei den Nambikwara Einzug gefunden, aber nicht als eine innere Entwicklung der eigenen Kultur, sondern als übernommene Symbole, deren Bedeutung ihnen verborgen blieb, und dies „eher im Hinblick auf ein soziales als auf ein intellektuelles Ziel“ ${ }^{66}$

Allein der Häuptling des Stammes, schien mehr erkannt zu haben und so hatte er dann bei jedem Treffen ein im Tausch erworbenes Notizbuch dabei, womit er den anderen gegenüber seine Überlegenheit kundtun wollte, aber auch zeigen wollte, dass er mit den Gästen gewisse Codes teilte, die den anderen fremd blieben. ${ }^{67}$ Der Häuptling tat so als wäre er der Sache kundig, als hätte er mit den deutlich überlegenen Besuchern ein Abkommen abgeschlossen. Seine Schlauheit und Gerissenheit war, das Schreiben als eine Form von Überlegenheit den anderen gegenüber zu inszenieren und somit auch eine Form von Macht. Diesem entnimmt Lévi-Strauss voreilig, dass die Schrift ein Mittel der Macht und der Unterdrückung der schwächeren Völker sein konnte, aber auch der Kontrolle und Macht im eigenen Volk. ${ }^{68}$

63 Vgl. Claude Lévi-Strauss: Traurige Tropen (1955). Köln 1974, S. 255-264. 64 Vgl. ebd., S. 258.

$65 \mathrm{Vgl}$. ebd.

66 Ebd., S. 261.

67 Vgl. ebd., S. 258-259.

68 Vgl. ebd., S. 263: Lévi-Strauss sieht den Zweck der Schrift primär als Ausbeutung und die schriftliche Mitteilung als Mittel Hilfsmittel zur Versklavung: „Es scheint somit, dass die Schrift zunächst der Ausbeutung des Menschen diente, bevor sie seinen Geist erleuchtete.“ Und ferner: „Wenn meine Vermutung richtig ist, so bestand also die primäre Funktion der schriftlichen Mitteilung darin, die Versklavung zu erleichtern.“ (Ebd.) 
Die Schrift, so schlussfolgert Lévi-Strauss, scheint eher die Unterjochung der Menschen zu fördern als deren Aufklärung oder Bildung. Das Schreiben sei ein Beitrag zur Entwicklung von Macht gewesen, ein Mittel, die Vergangenheit und Zukunft besser zu organisieren. So fällt, laut LéviStrauss, das Aufkommen der Schrift zusammen mit der Gründung von Städten und Reichen, mit der „Integration einer Großzahl von Individuen in ein politisches System und ihre Aufteilung in Kasten und Klassen“. 69

Die These, die der Anthropologe hier voraussetzt, ist, dass es einen naiven Urzustand gibt, dem durch die Schrift Gewalt angetan wird. Er nimmt an, dass es ein ursprüngliches und darin unbeflecktes Leben in Frieden und Eintracht gibt, das durch das Element der Schrift gestört wird. Die Geste des Häuptlings interpretiert er als Nachahmung im besten Wissen davon, dass er durch das Schreiben die anderen besser kontrollieren könnte. Lévi-Strauss unterstreicht somit die Dienlichkeit der geschriebenen Kommunikation, und zwar würde diese vor allem der Ausbeutung des Menschen dienen ${ }^{70}$ und zugleich diejenigen, die ihrer mächtig sind, stärken, „Prestige und Autorität" vergrößern. ${ }^{71}$ Der intellektuelle oder ästhetische Aspekt bleibt sekundär, ${ }^{72}$ ebenso die vervielfachte „Möglichkeit, Kenntnisse zu erwerben und zu bewahren.“"73

Derrida unternimmt in der Grammatologie eine Analyse des sozialen Faktors der Schrift, anhand dieser von Lévi-Strauss geschilderten ,Schreibstunde': die Schrift wird als Entfremdung betrachtet, die umso mehr entfremdend ist, als sie Verordnungen festhält, die soziale Gewalt und Macht ermöglichen. Derrida deckt in seiner Analyse des sozialen Faktors der Schrift, Vorurteile, die dieser Schilderung zugrunde liegen, auf, so etwa das Vorurteil, dass die Striche, die die Nambikwara aufzeichneten nicht auch schon Schrift sein könnten. ${ }^{74}$ Derrida geht es in seiner Bezugnahme zu dieser Schilderung hauptsächlich um die problematische Annahme bei Lévi-Strauss, dass der Zwischenfall den er schildert als Parabel dafür stehen kann, wie die Schrift Eingang bei Menschen findet und somit, wie sie ihren Ursprung hat. Zwar sieht Lévi-Strauss, dass es sich nicht eigentlich um eine Entstehung der Schrift bei einem Volk

69 Ebd., S. 263.

70 Vgl. ebd.

71 Ebd., S. 261.

72 Ebd., S. 263.

73 Ebd., S. 261.

74 Vgl. Derrida: Grammatologie (wie Anm. 41), S. 218. 
handeln kann, sondern vielmehr um die Übernahme von Symbolen. ${ }^{75}$ Dennoch schlussfolgert er, dass die Schrift auf die geschilderte Weise den Eingang zu diesem bestimmten Volk findet. ${ }^{76}$ Derrida kritisiert den Ansatz, zu schnell von sozial-politischen Faktoren auf die Entstehung der Schrift zu schließen, da er, erstens, die Idee eines Ursprungs der Schrift nicht billigen möchte und, zweitens, nicht zulassen möchte, dass die Entstehung der Schrift mit der erwähnten Übernahme von Symbolen verwechselt werden soll. ${ }^{77}$

Für uns hier wichtig ist allerdings genau der soziokulturelle Faktor der Schrift, der bei Lévi-Strauss sehr anschaulich beschrieben wird, auch wenn dieser, wie Derrida zu recht vermerkt, einseitig als negativ gewertet wird, so dass der Anschein erweckt wird „politische Gewalt“ könnte „nur Statthalter einer ungerechten Macht sein“, und somit allein die Unterjochung der Menschen hervorgehoben wird. ${ }^{78}$

Die Rolle, die der Schrift im soziokulturellen Zusammenhang zugewiesen wird, ist umstritten. ${ }^{79}$ Eine der Interpretationen der Rolle der Schrift ist mit Lévi-Strauss eine sozial-politische: Es gäbe keine Schrift, hätte diese keine soziale Bedeutung, und zwar in dieser Interpretation, jene der Beherrschung und der Kontrolle, Organisation etc. in einem Volk oder auch Staat, ob es sich nun um eine Potenzierung der Macht, die auch schon vom ungeschriebenen Wort ausgeübt werden kann, oder um eine neuartige Macht, über die die Schrift alleine verfügt, handelt. ${ }^{80}$ Schrift dient zwar der Kommunikation, ist aber nicht ausschließlich

75 Vgl. Lévi-Strauss: Traurige Tropen (wie Anm. 63), S. 261.

76 Vgl. ebd., S. 260.

77 Vgl. Derrida: Grammatologie (wie Anm. 41), S. 222.

78 Ebd., S. 230.

79 Für eine Gleichwertigkeit des Mündlichen und des Schriftlichen, die einander ergänzen, sowie den Vorzug der mündlichen Überlieferung in afrikanischen Kulturen vgl. Jacob Emmanuel Mabe: Denken mit dem Körper. Eine kleine Geistesgeschichte Afrikas. Nordhausen 2010, hier S. 90-94.

80 Derrida unterstreicht, dass Lévi-Strauss diesen Aspekt nicht gründlich durchdacht hat und somit das Spezifische der Schrift nicht ausgearbeitet hat. (Derrida: Grammatologie [wie Anm. 41], S. 223) Allerdings verlangt dieser Einwand nach einer Gründlichkeit, die Lévi-Strauss sich so gar nicht vorgenommen zu haben scheint. Er stellt deskriptiv fest, dass die Verbreitung der Schrift, wie weiter oben angeführt, mit der Entstehung von Städten und Reichen zusammenfällt. Damit lenkt er bereits die Aufmerksamkeit darauf, dass Schrift irgendwie mit Macht verwoben ist und es scheint ihm allein auf dieses Augenmerk anzukommen. 
dazu gedacht, sondern bekundet auch Macht und Überlegenheit. Diesen Aspekt weist Derrida auch nicht zurück: er unterstreicht allerdings, dass auch Aufzeichnungen, die für bildliche Darstellungen gehalten werden könnten, im Grunde Schemata sind, die soziale Strukturen oder Genealogien darstellen. ${ }^{81}$

Derrida fügt dieser Beschreibung bei Lévi-Strauss noch einen weiteren Aspekt hinzu: „Aufgrund bestimmter und sehr sicherer Informationen wissen wir heute, daß die Genese der Schrift (im geläufigen Sinn) fast überall und am häufigsten an die Sorge um die Genealogie gebunden war.“"82 Was er damit Lévi-Strauss vorhält ist, dass er die „Stricheleien und Zickzacklinien" ${ }^{83}$, mit denen diese die Kürbisse verzierten, nicht als Schrift zumindest in Frage gestellt hat, sondern die schlichte Übernahme von „Symbolen“" ${ }^{\text {"S4 }}$ so gedeutet hat, dass dadurch „die Schrift den Weg zu den Nambikwara gefunden" hat ${ }^{85}$ Dass derartige Aufzeichnungen, wie auch schon für bildliche Darstellungen gehaltene Schemata, soziale Funktion haben können, wird von Lévi-Strauss übersehen, so dass er die Entstehung der Schrift auf eine Übernahme der Schrift verlagert.

Die Frage nach dem Ursprung der Schrift als Entstehungsmoment steht für uns hier allerdings nicht im Mittelpunkt, sondern vielmehr die Frage nach dem sozialen Faktor der Schrift, als Veranschaulichung eines Bereichs in dem die Sinnstiftung und Weltbildung durch die Schrift vermerkt werden kann. Kann dieses Moment, das Lévi-Strauss darstellt, nicht auch als ein schlichter Hinweis darauf gelesen werden, die Schrift in ihren Differenzierungen auch als Teil der Lebenswelt zu verstehen und darin auch als sinnstiftend gelesen werden? Lévi-Strauss schlägt vor, die Schrift mit Blick auf die Unterjochung der Menschen zu verstehen und, anstatt diese Interpretation als einseitig abzulegen, wäre es doch denkbar, die Verbindlichkeit der Schrift in den Vordergrund zu bringen. Und wenn dem so ist, dann könnten die unterschiedlichen Systeme der Schriften zu unterschiedlichen Weisen der Verbindlichkeit führen, wobei Verbindlichkeit Verbundensein, Pflicht, Gesetz oder auch Nachhaltigkeit bedeuten kann. ${ }^{86}$

81 Vgl. Derrida: Grammatologie (wie Anm. 41), S. 218.

82 Ebd., S. 218.

83 Lévi-Strauss: Traurige Tropen (wie Anm. 63), S. 258

84 Ebd., S. 261.

85 Ebd., S. 260.

86 Ich wende hier den Begriff der Verbindlichkeit in einem sehr weiten Sinne an, gerade um das hier angeführte Gedankenexperiment in der Fort- 
Die Schriftlichkeit gilt als Träger sozial und kulturell sinnkonstitutiver Differenz, wenn die Schrift Bedeutungen auf die eine oder andere Weise gliedert und diese zwar sprachlich, aber verschieden von der Rede verfasst ${ }^{87}$ Dabei handelt es sich gerade nicht um die Medialität der Schrift als Vermittlung, sondern vielmehr um die Schrift als sinnstiftende. Wird der Begriff der Schriftlichkeit sehr weit gefasst, so ist schwer auszumachen, ob nicht auch schon zufällige Zeichen in der Umwelt wie Schrift gelesen werden. Ist dem so, dann wäre die Schrift als Medium so weit gefasst, dass der Sinn einer jeden gezielten Vermittlung kaum mehr nachvollziehbar bleibt. Daher ist die Vermittlung oder Medialität gerade untergraben. Schriftlichkeit prägt auch da, wo sie nicht als Schrift gegeben ist, sondern z. B. etwas in bildlichen Sinnstrukturen abzulesen gibt.

\section{SCHLUSSÜBERLEGUNGEN: DIE SINNSTIFTUNG DURCH DIE SCHRIFT}

Drei Hinweise darauf, wie die Sinnstiftung durch die Schrift in soziokulturellem Zusammenhang befragt werden könnte, sollen diesen Beitrag nun abrunden:

führung offen zu lassen. Ohne allzu weit auszuholen, möchte ich erneut die Frage aufwerfen, ob unterschiedliche Schriftarten und Schriftweisen, soziokulturell zu unterschiedlichen Formen der Verbindlichkeit führen, die in verschiedenen Schriftkulturen auch unterschiedlich ausgeprägt sind. Jan Assmann hält fest: „Die Folgen der Schriftkultur sind vielfältig und nehmen in den einzelnen Gesellschaften ganz verschiedene Richtungen. Dabei spielt aber die Struktur des Schriftsystems, ideographisch oder phonographisch, alphabetisch oder syllabisch, konsonantisch oder vokalisiert, eine eher untergeordnete Rolle. Entscheidend ist vielmehr das Zusammenspiel einer Vielzahl von Faktoren, die innerhalb der einzelnen Kulturen und Epochen in jeweils anderen Konfigurationen wirksam werden." (Assmann: Das kulturelle Gedächtnis, wie Anm.1, S. 301) Und dennoch, ließe sich nicht gerade aus morphomatischen Überlegungen heraus, genau in diesem Punkt, ergänzend, die Frage neu aufrollen? Umso mehr wäre unter diesem Aspekt auch auf die Frage nach der Wissenschaftlichkeit oder Objektivierung in der Schrift zu achten und auf die Lesbarkeit von Bildern und bildlichen Vermittlungen, sowie zur Beeinflussung der Ästhetik und der Kunst, worauf ich aber hier nicht mehr eingehen kann.

87 In diesem Sinne habe ich hier auch die „Schreibstunde“ von LéviStrauss gelesen, ohne gesondert auf die marxistischen Aspekte einzugehen. Vgl. hierzu Derrida: Grammatologie (wie Anm. 41), S. 209. 
Die Dauerhaftigkeit, Beständigkeit der Schrift ist eines der Argumente, wie Schrift sich eingebürgert hat, ein Argument, das sowohl der Aufzeichnungen zur Genealogie, als auch ihrer Interpretation als Machtträger zugrunde liegt. Allerdings wird die Dauerhaftigkeit in Bezug auf die Vergänglichkeit der gesprochenen Rede gedacht, das heißt in Bezug auf ein Flüchtiges: Sie bleibt dem phonetischen Logozentrismus verhaftet. Allein Dauerhaftigkeit würde auch den Stellenwert der Schrift, so wie Derrida diesen hervorhebt, nicht rechtfertigen, und auch der vergleichende Rückgriff auf die Stimme macht seine Thesen, wie gesehen, angreifbar. Dennoch setzt Derrida den Punkt, dass die Schrift, als Differenz, das Denken anders als die lebendige Rede gliedert, so dass der Rückbezug auf die gesprochene Rede nicht mehr gerechtfertigt ist, sondern eher die gesprochene Rede auf eine Ur-Schrift zurückzuführen ist. ${ }^{88}$ Festgehalten werden soll hier eine gewisse Vorrangigkeit der Struktur der Schrift, die unterschiedlich ausfallen dürfte. Die Universalität einer Ur-Schrift, so imateriell diese auch aufgefasst wird, ist spätestens mit dem ersten Schriftzug oder der ersten Zeichensetzung in Frage gestellt, da auch die Differenzierung strukturell in unterschiedlichen Kulturen auch unterschiedlich ausfallen dürfte.

Jenseits der materiellen Dauerhaftigkeit, die nur eine Form der Zeitigung der Schrift ausmacht, ist die Schrift auch aufgrund einer weiteren Sinnstruktur nicht auf die Rede zurückzuführen, nämlich der Kontinuität, worauf das Geschichtsdenken beruht. ${ }^{89}$ Aus dieser Linearität und

88 Roy Harris' Integrationismus zielt auf eine ähnliche sinnstiftende Funktion der Schrift ab, wobei eher auf Kommunikation wert gelegt wird. (vgl. Roy Harris: Intorduction to Integrational Linguistics. Oxford 1998). Hier meine ich nicht allein die Sinnstiftung durch im Handlungskontext geprägte Wörter, sondern einen Unterschied der auf der Ebene des Vergleichs von Rede und Schrift ansetzt. Die Schrift ist nicht lediglich ein Pendant zur Rede oder zum Denken, sondern sie schafft eigenständige Sinndimension: Es gibt eine Welt, die uns so nur als Schriftlichkeit begegnet. Romane, Werbesprüche etc. Und mehr noch: Die Sinnstruktur, die durch das Lesen erlernt wird, die in der Schriftlichkeit erfahren wird, z. B. eine Passage immer wieder zu lesen, zurückzublättern, nimmt Einfluss darauf, wie wir im Alltag die Welt erfahren.

89 Auch wenn die Schrift es gerade erlaubt, beim aktiven Lesen, auf früheres zurückzugehen, bzw. Text zu überspringen, so ist der Text als solcher doch eine immer wieder wiederholbare Linie, bei der auch frühere Texte von späteren unterschieden werden können. Es handelt sich also nicht um das Erlebnis des Lesens, sondern um das Verzeichnis der Texte als frühere oder 
Gleichzeitigkeit heraus, wird die Zeiterfahrung mit der Schrift eine andere als in der Rede. Während die Rede nur in der Präsenz möglich ist, in der die Rede gesprochen und gehört wird, aber als solche kaum im Detail wiederholt werden kann, zeitigt die Zeitlichkeit des Geschriebenen alle drei Zeitformen Gegenwart, Vergangenheit und Zukunft: als gegenwärtiges Lesen des früher Geschriebenen - das als solches, in der Schrift verankertes, unverändert immer wieder aufrufbar ist - und zukünftig Verstandenem, Erschlossenem, in einen Verstehenshorizont Integriertem, wobei gerade in dieser zeitlichen Struktur die Verbindlichkeit zu verankern wäre.

Um nun aber einen Schritt weiter zu gehen, wäre zu vermerken, dass die Erfahrung der Schrift, das Lesen als solches, wie jede Erfahrung, Spuren im Zusammenhang der weiteren Erfahrungen hinterlässt. So wie unterschiedliche Sprachen auch unterschiedliche Strukturen der Welt zeigen, da sie unterschiedliche Gliederungen und Zusammenhänge beinhalten, und das jenseits des übersetzbaren Sinngehalts (also gerade das nicht Übersetzbare), so weisen auch Schriftarten unterschiedliche Gliederungen auf, die als Gliederungen wiederum in Sinn umschlagen. Wenn der Sinn einen gewissen Zusammenhalt von Bedeutungen meint, dann kommt es auf die Weise an, wie dieser Zusammenhalt gegliedert ist, um den Sinn nachvollziehen zu können. In dieser Weise, als Gliederungsmöglichkeit, die nicht nur von der Sprache vorgegeben ist, sondern auch von der Schrift, insbesondere durch die wiederholte Übung des Lesens, könnte die Schrift den Sinn prägen. Ein Wink dabei wäre, die Lesbarkeit der Welt hervorzuheben, die wesentlich an der Schriftlichkeit orientiert ist..$^{90}$

spätere. Für eine Analyse des früher und später im Lesen des Textes, wobei die Erfahrung eines Textes gerade nicht die Linearität der gesprochenen Rede voraussetzt, sondern eben Sprünge ermöglicht, vgl. Günter Figal: Verstehensfragen. Studien zur phänonneologisch-hermeneutischen Philosophie. Tübingen 2009, S. 109. Figal unterstreicht eine „eidetische Dimension der Sprache“ die sich beim Lesen als Erfahrung im „Verweilen“ zwischen dem „Präsenten“ des Texts, auf den man immer wieder zurückkommen kann, und der „Präsenz“ des Gelesenen im Gedächtnis (ebd., S. 110).

90 Vgl. Hans Blumenberg: Die Lesbarkeit der Welt. Frankfurt a. M. 1986, S. 10. Blumenberg setzt den Gedanken der Lesbarkeit der Welt nicht bei der Schriftlichkeit als solcher an, sondern vielmehr bei der Kultur des Buches, im Vergleich der Welt mit einem Buch. Für uns hier ist der Gedanke bedeutsam, dass die Sinnstruktur, die aus dem Schriftlichen gekannt ist, auch in die Erfahrung der Welt übertragen wird. Sicherlich kommt es dabei darauf an, dass die Schrift überhaupt schon gekannt ist und dass auch gewisse Formen des Schreibens vertraut sind. Aber gerade diese Frage stellt 
In ihrer Lesbarkeit zeigt die Welt andere Facetten als in ihrer Sagbarkeit, auch wenn nicht zuletzt das Gelesene in Gesagtes umgesetzt wird. Die Lesbarkeit meint einen Überschuss von Sinn, der im Sagen nie ganz erschlossen werden kann. Die sinnstiftende Gleichzeitigkeit des beim Lesen Gegebenen kann im Sagen nur nachvollzogen werden, z. B. wenn der Zusammenhang eines Landschaftsbildes ,gelesen' wird, dann aber als Gelesenes und somit Gesagtes in seine einzelnen Momente zerfällt.

Jenseits der Buchstaben, die als verwendbares ,Zeug' gesehen werden könnten, und jenseits der Frage nach dem Ursprung der Schrift, ist die Schrift als soziokulturelles Phänomen zu betrachten, das auf unsere Erfahrung der Welt und somit auf die Sinnbildung Einfluss nimmt. Eine mögliche Weise, diese Änderung der Erfahrung zu verstehen, wäre, die Schrift als Machtinstrument aufzufassen, wie hier mit Lévi-Strauss gezeigt, wobei daran ersichtlich wird, dass es sich hierbei um Sinnstiftung im Sinne der Veränderungen der soziokulturellen Strukturen handelt. Allerdings bleibt diese Perspektive nur ein Beispiel. Die Frage, wie die Schrift im Weiteren die Erfahrung bestimmt, bleibt in der Frage nach der Zeitigung der Schrift verankert, die mit den weiter oben formulierten Hinweisen gestellt wird. Aus dieser Perspektive wäre dann auch die Rolle der Buchstaben im Vergleich zu anderen möglichen Schriftarten, wie etwa die Bilderschrift, zu besprechen.

sich in der heutigen Erfahrung der Welt eben immer weniger. Und wenn Analphabetismus noch vor nicht allzu langer Zeit ein schwerwiegendes Problem gewesen ist, stellt sich sicherlich die Frage, wie die Erfahrung der Welt durch den Alphabetismus verändert wurde und noch verändert werden kann, und mehr noch, ob unterschiedliche Schriften auch zu unterschiedlichen Erfahrungen führen können. „Von der Welt Erfahrung zu machen, wie man sie in einem Buch oder in einem Brief verdanken kann, setzt nicht nur den Alphabetismus, nicht nur die Vorprägung der Wünsche auf Sinnzugang durch Schrift und Buch voraus, sondern auch die kulturelle Idee des Buches selbst, insofern es nicht mehr bloßes Instrument des $\mathrm{Zu}$ ganges zu anderem ist. Ist es aber zu einer eigenen Erfahrung von Totalität autonomisiert, wie exemplarisch im frühgriechischen Epos oder im Buch der Bücher, tritt die Bucherfahrung in Rivalität zur Welterfahrung.“ (Ebd.) 



\section{BEITRÄGERINNEN UND BEITRÄGER}

RalF BeUthan Prof. Dr., Philosoph am Department of Philosophy der Myongji Universität Seoul, Südkorea

STEFAN CLAUDIUS Diplom-Designer, Typograf und Schriftgestalter im Büro „Sichtvermerk“ in Mülheim an der Ruhr, seit 2002 Betreiber der Cape Arcona Type Foundry, 2010 bis 2012 Gastprofessor an der Hochschule für Bildende Künste in Hamburg

EVELINE CIOFLeC Dr., Philosophin, Postdoc-Fellow in der School of Religion, Philosophy and Classics der Universität KwaZulu-Natal, Südafrika

YVONNE FÖRSTER-BEUTHAN Prof. Dr., Philosophin am Institut für Philosophie und Kunstwissenschaft der Leuphana Universität Lüneburg

NORIKO KAYA Prof. Dr., Kalligraphin an der Faculty of Art Education der Osaka Kyoiku Universität, Japan

RYOZO MAEDA Prof. Dr., Literaturwissenschaftler am Department of German Languages, Literature and Cultural Studies der Rikkyo Universität, Japan

MATHIAS OBERT Prof. Dr., Philosoph am Institute of Philosophy der National Sun Yat-sen Universität, Taiwan

RYÔSUKE ÔHASHI Prof. Dr., Philosoph und Fellow (2010-11) am Internationalen Kolleg Morphomata der Universität zu Köln, zur Zeit Fellow am Forschungsinstitut für Philosophie Hannover

BEATRICE PRIMUS Prof. Dr., Linguistin am Institut für deutsche Sprache und Literatur I der Universität zu Köln 
MARTIN ROUSSEL Dr., Literaturwissenschaftler, Wissenschaftlicher Geschäftsführer des Internationalen Kollegs Morphomata, Lehrauftrag am Institut für deutsche Sprache und Literatur der Universität zu Köln I

CHRISTIAN STETTER Prof. Dr., emeritierter Sprachwissenschaftler am Institut für Sprach- und Kommunikationswissenschaft der RWTH Aachen und Geschäftsführer von semantics Kommunikationsmanagement $\mathrm{GmbH}$

HELWIG SCHMIDT-GLINTZER Prof. Dr., Sinologe, Direktor der Herzog August Bibliothek Wolfenbüttel

LUTZ WAGNER Dr. rer. pol., forscht privat auf dem Gebiet der Alphabete der Welt 
Bislang in der Morphomata-Reihe erschienen:

1 Günter Blamberger, Dietrich Boschung (Hrsg.), Morphomata. Kulturelle Figurationen: Genese, Dynamik, Medialität, 2011. ISBN 978-3-77055148-4.

2 Martin Roussel (Hrsg.), Kreativität des Findens. Figurationen des Zitats, 2012. ISBN 978-3-7705-5305-1.

3 Jan Broch, Jörn Lang (Hrsg.), Literatur der Archäologie. Materialität und Rhetorik im 18. und 19. Jahrhundert, 2012. ISBN 978-3-77055347-1.

4 Dietrich Boschung, Corina WesselsMevissen (Hrsg.), Figurations of Time in Asia, 2012. ISBN 978-37705-5447-8.

5 Dietrich Boschung, Thierry Greub, Jürgen Hammerstaedt (Hrsg.), Geographische Kenntnisse und ihre konkreten Ausformungen, 2012. ISBN 978-37705-5448-5.

6 Dietrich Boschung, Julian Jachmann (Hrsg.), Diagrammatik der Architektur, 2013. ISBN 978-3-7705-5520-8.
7 Thierry Greub (Hrsg.), Das Bild der Jahreszeiten im Wandel der Kulturen und Zeiten, 2013. ISBN 978-3-7705-5527-7.

8 Guo Yi, Sasa Josifovic, Asuman LätzerLasar (Hrsg.), Metaphysical Foundation of Knowledge and Ethics in Chinese and European Philosophy, 2014. ISBN 978-3-7705-5537-6.

9 Wilhelm Voßkamp, Günter Blamberger, Martin Roussel (Hrsg.), Möglichkeitsdenken. Utopie und Dystopie in der Gegenwart, 2013, ISBN 9783-7705-5554-3.

10 Dietrich Boschung, Sebastian Dohe (Hrsg.), Meisterwerk als Autorität. Zur Wirkmacht kultureller Figurationen, 2013. ISBN 978-3-77055528-4.

11 Stefan Niklas, Martin Roussel (Hrsg.), Formen der Artikulation. Philosophische Beiträge zu einem kulturwissenschaftlichen Grundbegriff, 2013. ISBN 978-3-7705-5608-3 
Die Morphomata-Reihe wird herausgegeben von Günter Blamberger und Dietrich Boschung.

Das Internationale Kolleg Morphomata: Genese, Dynamik und Medialität kultureller Figurationen wird vom Bundesministerium für Bildung und Forschung im Rahmen der Initiative ,Freiraum für die Geisteswissenschaften' als eines der Käte Hamburger Kollegs gefördert. Jährlich bis zu 10 Fellows aus aller Welt forschen gemeinsam mit Kölner Wissenschaftlern zu Fragen kulturellen Wandels. Im Dialog mit internationalen Wissenschaftlern gibt das Kolleg geisteswissenschaftlicher Forschung einen neuen Ort - ein Denklabor, in dem unterschiedliche disziplinäre und kulturelle Perspektiven verhandelt werden.

www.ik-morphomata.uni-koeln.de

Ryôsuke Ôhashi war zuletzt Professor für Philosophie an der buddhistischen Ryukoku-Universität in Kyoto. Er ist Ehrendirektor des Nishida Kitaro Museum of Philosophy in Kahoku-shi, war u. a. Fellow am Wissenschaftskolleg in Berlin und am Internationalen Kolleg Morphomata. 1990 erhielt er den Philipp Franz von Siebold-Preis. - Seine Forschungen auf den Gebieten der Philosophie und Ästhetik leisten einen Beitrag zur interkulturell-philosophischen Verständigung.

Martin Roussel ist Wissenschaftlicher Geschäftsführer des Internationalen Kollegs Morphomata. Lehrt am Institut für deutsche Sprache und Literatur I der Universität zu Köln. 2007 Promotion mit einer Arbeit über Robert Walsers Mikrographie. Vorstandsmitglied der Heinrichvon-Kleist-Gesellschaft. - Veröffentlichungen insbesondere zur Literatur vom I8. bis 2I. Jahrhundert (u.a. Kleist, Karl May, Musil) und zu Schriftkulturen. 

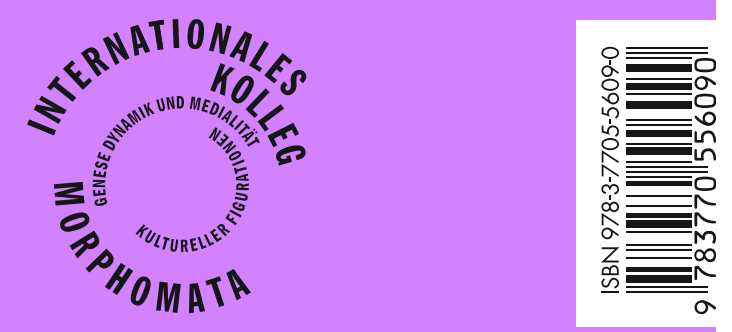

WILHELM FINK 